Portland State University

PDXScholar

$1-1-1986$

\title{
Elderly stroke patients and their partners: a longitudinal study of social support and well-being changes associated with a disabling stroke
}

Marie Therese Rau

Portland State University

Follow this and additional works at: https://pdxscholar.library.pdx.edu/open_access_etds Let us know how access to this document benefits you.

Recommended Citation

Rau, Marie Therese, "Elderly stroke patients and their partners: a longitudinal study of social support and well-being changes associated with a disabling stroke" (1986). Dissertations and Theses. Paper 478. https://doi.org/10.15760/etd.478

This Dissertation is brought to you for free and open access. It has been accepted for inclusion in Dissertations and Theses by an authorized administrator of PDXScholar. Please contact us if we can make this document more accessible: pdxscholar@pdx.edu. 
ELDERLY STRORE PATIENTS AND THEIR PARTNERS:

A LONGITUDINAL STUDY OF SOCIAL SUPPORT AND

WELL-BEING CHANGES ASSOCIATED

WITH A DISABLING STROKE

by

MARIE THERESE RAU

A dissertation submitted in partial fulfillment of the requirements for the degree of

DOCTOR OF PHILOSOPHY

in

URBAN STUDIES

Portland State University

(C) 1986 Marie T. Rau 
TO THE OFFICE OF GRADUATE STUDIES AND RESEARCH:

The members of the Committee approve the dissertation of Marie T. Rau presented Apri1 11, 1986.
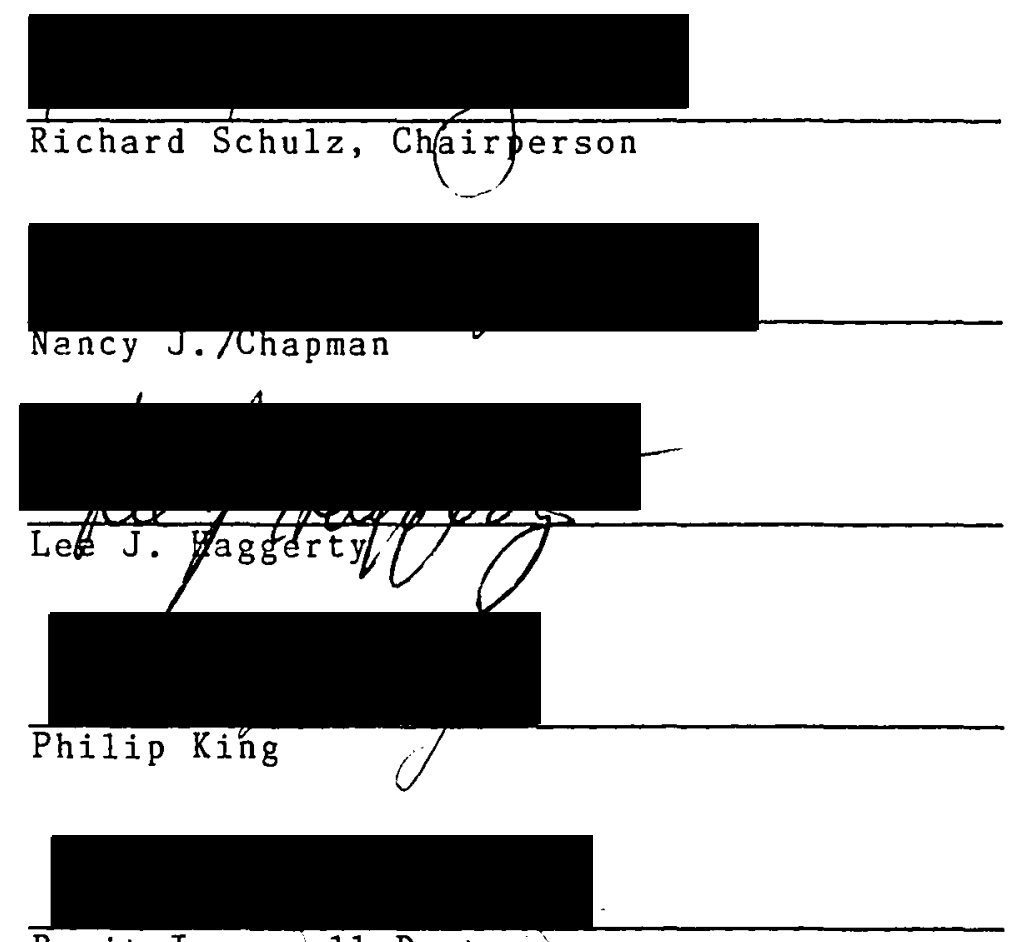

Berit Ingersol1-Dayton:

APPROVED :

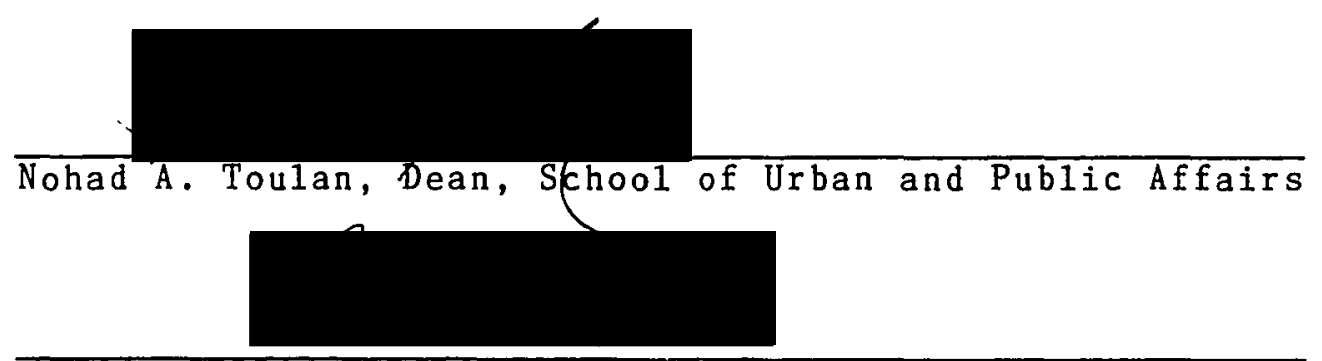

Bernard Ross, Dean of Graduate Studies and Research 
AN ABSTRACT OF THE DISSERTATION OF Marie T. Rau

for the Doctor of Philosophy in Urban Studies

presented April 11, 1986.

Title: Elderly Stroke Patients and Their Partners: A Longitudinal Study of Social Support and We11-Being Changes Associated with a Disabling Stroke.

APPROVED BY MEMBERS OF THE DISSERTATION COMMITTEE:

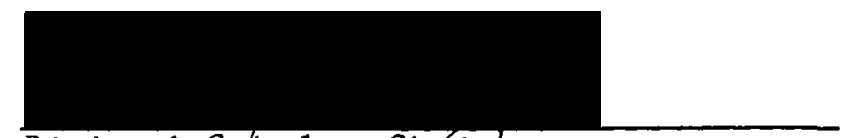

Richard Sqhulz, Chairperson
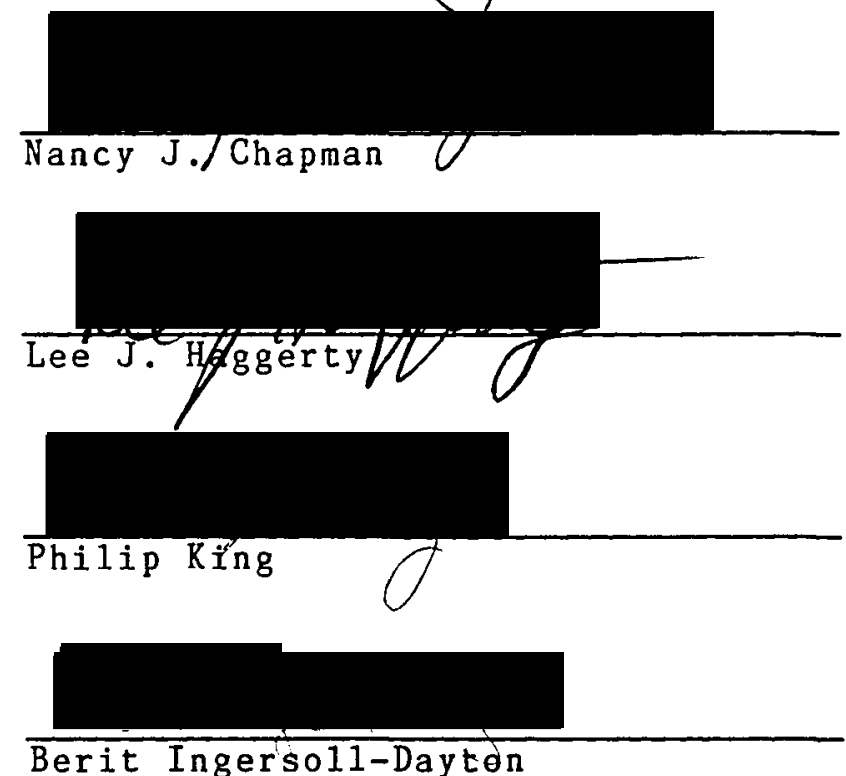

Social support has been suggested as an important factor in adaptation to illness and chronic disability. Current interest in the role of support in coping with chronic illness has been stimulated by the emergence of chronic disease and disability as the major U. S. health problem of the 1980's. One chronic disease which 
exemplifies the psychosocial impacts of disability is cerebrovascular accident, or stroke, the third leading cause of disability among middle-aged and older persons in this country.

Previous research on the impacts of stroke is largely descriptive and cross-sectional. There is a need for a longitudinal study of the process of adaptation to a stroke which includes multidimensional measures of social network characteristics; support provided $b_{y}$ the network; individual coping mechanisms; and health and well-being outcomes.

This investigation employed survey research methodology and a panel design to explore the relationship of social network changes, social support, stroke-related factors, individual characteristics, and demographic factors to depressive symptomatology and positive and negative wellbeing. Subjects were 50 elderly individuals who had recently suffered a first, completed stroke and their partners. Data were gathered at two points in time, with interviews scheduled six months apart. The primary datagathering tool was a questionnaire administered during structured, face-to-face interviews. Outcome measures included the CES-D depression scale and the Index of Psychological Well-Being. Data were analyzed using descriptive statistics, correlational procedures, multiple regression, and change-focused regression analyses.

Half of the caregivers and one-fourth of the 
respondent patients had depressive symptom scores above the cutoff level indicating high risk for depression. For the caregivers, lower depression levels at Time 1 were associated with better subjective health, less concern about being able to care for the patient in the future, higher levels of ADL functioning in the patient, greater perceived instrumental support in the pre-stroke network, and greater patient optimism.

At Time 2, lower caregiver depression scores were associated with lower levels of perceived burden, fewer health problems or negative changes in health status, fewer negative network interactions, greater network density, greater frequency of network contacts, and fewer perceived personality and behavior changes in the patient.

The best predictors of depression score for the caregivers at Time 1 were subjective health rating, the patient's level of functioning in activities of daily living (ADL), degree of concern about ability to care for the patient in the future, the proportion of the network providing instrumental support, and the percent of reciprocal confiding relationships reported. Together these variables accounted for $47 \%$ of the variance in depression score. At Time 2, the best predictors of depression were level of perceived caregiver burden, objective health score, and network density. Almost $50 \%$ of the variance in depressive symptoms was explained by these variables. The 
best predictor of caregiver depression level over time was Time 1 depression level. Feelings of burden and the proportion of reciprocal confiding relationships at Time 2 also were predictive of depression score after controlling for initial depression level health factors, and the patient's level of functional independence.

For the patients, higher depression scores at Time 1 were associated with whether they felt they could have done anything to prevent the stroke, higher levels of concern about their partner's ability to care for them in the future, and greater reported frequency of disagreement with their partners. At Time 2, higher levels of depressive symptomatology were associated for the patients with decreased satisfaction with amount of social contact, a greater proportion of friends in the post-stroke network, a greater degree of perceived negative health change, and change in employment status. Several of the social support measures which correlated with depression score suggested both positive and negative components of network support following a stroke.

The results of this study indicate that families coping with a stroke require support and intervention over a longer period of time than current health care policies recognize. These data will be valuable in designing strategies to minimize the negative psychosocial impacts of a stroke on both the patient and the caregiver. 


\section{ACRNOWLEDGEMENTS}

I want to thank the members of my dissertation committee for their helpful suggestions and their continued willingness to share their individual areas of expertise. I am especially indebted to my advisor, Dr. Richard Schulz, for his encouragement, enthusiasm, and support throughout this project.

The successful completion of this study owes much to many people. I want to acknowledge the assistance I received from the staff members of the participating hospitals and rehabilitation centers, especially Emanuel, Good Samaritan, and Providence Hospitals in Portland, and Salem General Hospital in Salem. A few individuals went "above and beyond" in referring potential subjects to the study, and I am grateful to them: Virginia Hendrickson, Anne Osborne, and Pat Stout at Emanuel Rehabilitation Center; Dianne Carlson and Kathy Manning at the Rehabilitation Institute of oregon, and Sandy Starch at Salem General.

A special thank you to my colleagues in speech Pathology at the Veterans Administration Hospital in Portland, Dr. Robert Marshall and Dr. Lee Ann Golper, for their support and encouragement on an almost daily basis, and because they understood that, at times, priorities lay elsewhere.

I would like to acknowledge the generous assistance of 
the Portland V. A. Library staff who were always there when I needed them. I also appreciate the helpful suggestions of Dr. Larry Binder and Dr. Muriel Lezak, Neuropsychology, and their continued interest in the project. A note of thanks to Dr. Bruce Coull, Neurology, for his assistance in the interpretation of the medical data.

To Charlene Rhyne and Alice Scannell, expert interviewers, for their input throughout the project and for their commitment to obtaining quality data. To my friend and colleague Connie Tompkins for her assistance with some of the data analysis.

I am especially grateful to the very special subjects of this study, the patients and their families who welcomed us into their homes, and who were willing to share their experiences and concerns for the potential benefit of future stroke families.

Finally, to my husband, John Rau, for sustaining me and encouraging me to learn and to grow. 
TABLE OF CONTENTS

PAGE

ACRNOWLEDGEMENTS . . . . . . . . . . . . . . . . . iii

LIST OF TABLES . . . . . . . . . . . . . . . . . . viii

LIST OF FIGURES . . . . . . . . . . . . . . . . xiv

CHAPTER

I. INTRODUCTION AND REVIEW OF THE LITERATURE • 1

Introduction . . . . . . . . . . 1

Literature Review . . . . . . . . 7

II. CONCEPTUAL FRAMEWORK OF THE STUDY . . . . . 73

Factors Predictive of Stroke Outcome . . 73

A General Model for the Study . . . . 84

Questions Addressed by the Study . . . 86

Hypotheses to be Tested . . . . . 88

III. METHODOLOGY 93

The Semple... . . . . . . . . 93

Data Collection . . . . . . . . 104

Human Subjects Assurances and Concerns . 109

Data Collection Instruments . . . . . 110

Operational Definitions . . . . . . 123

Data Analysis Procedures . . . . . . 136

IV. DESCRIPTIVE ANALYSIS: THE SAMPLE . . . . . 139

Demographic Characteristics of the
Sample . . . . . . . . . . . 139

Organizational Memberships and
Activities . . . . . . . . . . . 146

Health Status . . . . . . . . . 148 
Perceptions of the stroke . . . . . 151

Post-Stroke Changes . . . . . . . . 155

Conclusions . . . . . . . . . 160

V. DESCRIPTIVE ANALYSIS: SOCIAL NETWORKS AND

SOCIAL SUPPORT . . . . . . . . . 162

Subjects' Overall Social Networks . . . 162

Subjects' Intimate Social Networks . . . 184

Social support . . . . . . . . . 202

Summary: Intimate Network Social support and Social contacts . . . . . . 225

VI. CORRELATES AND PREDICTORS OF DEPRESSION AND WELL-BEING: TIME 1 AND TIME 2 . . . . . 228

Time 1... . . . . . . . . . . 229

Correlates of Depression and Well-Being

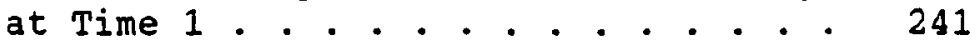

Regression Analyses, Time 1 . . . . . . 259

Time 2. . . . . . . . . . . . 278

Correlates of Depression and Well-Being at Time 2 . . . . . . . . . . 272

Regression Analyses, Time 2 . . . . 290

Summary: Correlates and Predictors of

Well-Being, Time 1 and Time $2 . \cdot 301$

VII. CHANGES IN DEPRESSION AND WELL-BEING OVER

TIME . . . . . . . . . . . . . 304

Descriptive and Correlational Analysis of Change Over Time....... . 304

Regression Analyses . . . . . . . . 312

Summary: Change Over Time . . . . . . 328 
vii

CHAPTER

PAGE

VIII. DISCUSSION AND IMPLICATIONS OF THE STUDY . . 330

Discussion . . . . . . . . . . 330

Implications of the study . . . . . . 350

REFERENCES . . . . . . . . . . . . . . . 356

APPENDIX

A Initial Interview Schedules . . . . . . 376

B Follow-up Interview Schedules . . . . . . 440

C Other Measures .............. 480

D Informed Consent . . . . . . . . . . 493 
LIST OF TABLES

TABLE

PAGE

I Structural and interactional social network characteristics and their definitions . .

II Factors predictive of stroke patient we11being . . . . . . . . . . . . 75

III Factors predictive of caregiver well-being . 78

IV Factors related to the stroke patientcaregiver relationship..... . . . 83

$V$ Number and percent of excluded stroke cases by reason for exclusion, Portland Stroke Study

VI Number and percent of dyads declining to participate by reason expressed, Portland stroke Study

VII Comparative demographic data for marital dyad sample and group which declined to participate . . . . . . . . . . . . .

VIII Comparative demographic data for marital dyad sample, 1979 Oregon CHSP sample, and threestate CHSP sample. . . . . . . . . 102

IX Total number and percent of referrals by referral source, Portland Stroke Study . . . . . 106

$\mathrm{X}$ Number and percent of referrals by source of referral, marital dyad sample . . . . . 107 
XI Scoring matrix for the Index of Psychological Well-Being . . . . . . . . . . . . 117

XII General demographic data: stroke patients . . 140

XIII General demographic data: caregivers . . . . 141

XIV General demographic data: dyads . . . . . . 145

XV Pre-stroke levels of socializing activities:

stroke patients and their partners . . . . 149

XVI Stroke patients' perceptions of the stroke . . 152

XVII Caregivers' perceptions of the stroke . . . . 154

XVIII Caregivers' overall pre-stroke social networks 164

XIX Caregivers' overall social networks, early post-stroke period . . . . . . . . . 167

XX Caregivers' overall social networks, Time 2 . 169

XXI Stroke patients' pre-stroke overall social networks ............... . 171

XXII Stroke patients' overall social networks, Time 2 . . . . . . . . . . . . . . . .

XXIII Non-respondent stroke patients' pre-stroke overall social networks . . . . . . . 176

XXIV Non-respondent stroke patients' Time 2 overall social networks . . . . . . . . . . 177

XXV Changes in the overall social networks of stroke patients from Time 1 to Time 2 . . . . . 183 
XXVI Characteristics of subjects' intimate social networks . . . . . . . . . . . . . 186

XXVII Gender composition of subjects' intimate social networks . . . . . . . . . . . . . . 187

XXVIII Caregivers' intimate social network composition by type of relationship . . . . . . . . .

XXIX stroke patients' intimate social network composition by type of relationship . . . . .

XXX Caregivers' Erequency of network contacts, Time 1 and Time 2. . . . . . . . . .

XXXI Patients' frequency of network contacts. Time 1 and Time 2. . . . . . . . . . . 196

XXXII Interconnectedness within and between respondents' networks . . . . . . . . .

XXXIII Caregivers' social support exchanges, Time 1 and Time 2. . . . . . . . . . . . 205

XXXIV Patients' social support exchanges, Time 1 and Time 2. . . . . . . . . . . . 207

XXXV Reciprocity in caregivers' helping networks • 212

XXXVI Reciprocity in patients' helping networks . • 213

XXXVII Direction of reciprocity in caregivers'

networks . . . . . . . . . . . . . .

XXXVIII Direction of reciprocity in patients' networks 
xi

TABLE

PAGE

XL Respondents expressed satisfaction with overall amount and quality of social network contact 223

XLI Mean values for selected caregiver variables . 230

XLII Mean values for selected patient variables . . 231

XLIII Comparative distribution of scores on the Index of Psychological Well-Being for a community sample and the stroke study sample . . . 235

XLIV Correlations with CES-D for caregivers, Time 1242

XLV Correlations with CES-D for patients, Time 1 - 243

XLVI Correlations with positive well-being for caregivers and patients, Time 1 . . . . . 247

XLVII Correlations with negative well-being for caregivers, Time 1 . . . . . . . . . 250

XLVIII Correlations with negative well-being for patients, Time 1 . . . . . . . . . . 251

XLIX Intercorrelations among outcome variables at Time 1 . . . . . . . . . . . . 256

L Hierarchical regression for CES-D, Time 1:

All caregivers included . . . . . . . 261

LI Hierarchical regression for CES-D, Time 1:

Female caregivers only . . . . . . . . 264

LII Hierarchical regression for positive well-

being, Time 1: All caregivers included . . 266 
LIII Hierarchical regression for positive wellbeing, Time 1: Female caregivers only . . 267

LIV Hierarchical regression for negative wellbeing, Time 1: All caregivers included . . 269

LV Hierarchical regression for negative wellbeing, Time 1: Female caregivers only . . . 271

LVI Correlations with CES-D for caregivers, Time 2 . . . . . . . . . . . . . .

LVII Correlations with CES-D for patients, Time 2. . . . . . . . . . . . . . 278

LVIII Correlations with positive well-being, Time 2 . 284

LIX Correlations with negative well-being for caregivers, Time 2 . . . . . . . . 286

LX Correlations with negative well-being for patients, Time 2............ 287

LXI Intercorrelations among outcome variables at Time 2 . . . . . . . . . . . . . 291

LXII Hierarchical regression for CES-D, Time 2:

All caregivers included . . . . . . . . 293

LXIII Hierarchical regression for CES-D, Time 2:

Female caregivers only . . . . . . . 295

LXIV Hierarchical regression for positive wellbeing, Time 2: All caregivers included . . 296 
xiii

TABLE

PAGE

LXV Hierarchical regression for positive well-

being, Time 2: Female caregivers only . . . 298

LXVI Hierarchical regression for negative well-

being, Time 2: All caregivers included . . 300

LXVII Hierarchical regression for negative well-

being, Time 2: Female caregivers only . . 302

LXVIII Hierarchical regression for CES-D, Time 1

and Time 2: all caregivers . . . . . . 315

LXIX Hierarchical regression for CES-D, Time 1

and Time 2: Female caregivers only . . . . 318

LXX Hierarchical regression for positive well-

being, Time 1 and Time 2: All caregivers . 320

LXXI Hierarchical regression for positive well-

being, Time 1 and Time 2: Female

caregivers only . . . . . . . . . . 321

LXXII Hierarchical regression for negative well-

being, Time 1 and Time 2: All caregivers . 324

LXXIII Hierarchical regression for negative well-

being, Time 1 and Time 2: Female

caregivers only . . . . . . . . . . . 327

LXXIV Summary of social network and social support

measures obtained in the study, Time 1 and

Time 2. . . . . . . . . . . . . 335 


\section{LIST OF FIGURES}

FIGURE

PAGE

1. General model for the study . . . . . . . .

2. Caregivers' overall social networks by selected relationship categories for three points in time

3. Stroke patients' overall social networks by selected relationship categories for three points in time..............

4. Non-respondent stroke patients' overall social networks by selected relationship categories for two points in time .......... 180

5. Caregivers' intimate social networks by selected relationship variables for two points in time . . . . . . . . . . .

6. Stroke patients' intimate social networks by selected relationship variables for two points in time . . . . . . . . . . . . 
CHAPTER I

INTRODUCTION AND REVIEW OF THE LITERATURE

INTRODUCTION

Social support has long been suggested as one of the factors important in the prevention of, recovery from, and adaptation to illness and chronic disability. Scientific interest in the psychological and physical health benefits of being socially integrated has existed for almost as long as researchers have been concerned about the possible negative effects of industrialization, urbanization, and other life stresses on human well-being and sense of community (Durkheim, 1951; Toennies, 1957; Wirth, 1938). The concept of "social support" emerged formally in the 1970 's as one of several possible mediating variables to explain why life stresses do not consistently and uniformly produce negative health outcomes among similarly stressed individuals (Antonovsky, 1979; Caplan, 1974; Cassel, 1974; 1976; Cobb, 1976). Investigators have observed that those who have and maintain ties to other persons or who are provided with specific types of social support (Ressler \& McLeod, 1985) fare better than those who do not in terms of mortality (Berkman \& Syme, 1979; Blazer, 1982; House, Robbins, \& Metzner, 1982), mental health (Henderson \& Byrne, 1982; Schaefer, Coyne, \& Lazarus, 1981; Wilcox, 1981), physical health (Marmot \& Syme, 1976; Medalie \& Goldbourt, 
1976; Nuckolls, Cassel, \& Kaplan, 1972), and adaptation to and recovery from physical illness (Berle, Pinsky, Wolf, \& Wolff, 1952; Dimond, 1979; Dunkel-Shetter, 1984; Finlayson, 1976; Funch \& Marshall, 1983; Robertson \& Suinn, 1968). Nevertheless, there remain many unanswered questions, especially about the role of specific social support processes in coping with illness and disability and about causal relationships between support, stressors, individual coping mechanisms, and illness adaptation.

Current interest in the importance of social support in recovery from illness and in the possibility of supportive interventions with chronically ill and disabled persons has been motivated by several factors. First has been the emergence of chronic disease and disability as the major U. S. health problem of the 1980's. Chronic diseases now account for eight of the ten leading causes of death in the United states, including the first three - heart disease, cancer, and stroke (Matarazzo, 1982). The rate of death from chronic disease in this country in 1980 was nearly 70 per 100, while deaths from infectious diseases were 6 per 100 (Matarazzo, 1982). In contrast, at the turn of the century, United States morbidity and mortality rates were largely related to infectious diseases, such as influenza, pneumonia, and diphtheria.

second, there are increasing numbers of persons over the age of 65 in our population, the age group with the 
greatest number of chronic disabling illnesses (strauss \& Glaser, 1975). This has resulted in a greater awareness of the relationship between chronic illness and psychosocial and health care costs. Chronic diseases do not resolve or result in death within a relatively short time period, as is the case with infectious diseases. Chronic illness typically lingers for the rest of a person's lifetime, causing great suffering, family life disruption, personal financial hardship, and tremendous costs to society as a whole (Stachnik, Stoffelmayr, \& Hoppe, 1983).

Third, the importance of approaches other than just medical to the management of chronic diseases has been recognized (Burish \& Bradley, 1983; Feldman, 1974; Strauss \& Glaser, 1975). The psychosocial impacts of chronic disease on patients and families have focused attention on the adaptation and coping aspects of chronic illness. The individual with a life-threatening or chronic, disabling illness experiences a number of psychological stresses (Moos \& Tsu, 1977). Strain (1979) has suggested what some of these psychologic reactions to chronic medical illness might be. Chronically ill persons perceive a threat to selfesteem and body intactness that challenges their beliefs that they are masters of their own bodies. There may be a fear of loss of love and approval that evolves from concerns that their illness and dependence on others will cause significant others to withdraw. There is fear of loss of 
control of body functions and/or parts with resulting loss of independence. During the acute phase or phases of the illness there is anxiety resulting from separation from loved ones and the familiar environment which provided support, gratification, and a sense of intactness. There may be guilt and fear of retaliation for having incurred the health problem in the first place. Concern about strangers providing intimate care may be present.

Miller (1983), in identifying a typology of coping tasks faced by the chronically ill or disabled, lists one of these as "adjusting to altered social relationships." Why might chronic illness result in social isolation and loneliness? In the first place, the sick or disabled individual may reduce social contacts because of depleted energy reserves, or poor self-concept. Also, thought processes may be dominated by the illness or chronic condition. Only the individual's close family and most loyal friends may persist in being supportive during such a repetitive pattern of interaction. Finally, isolation may be initiated by the withdrawal of significant others because they feel uncomfortable or unsure of how to respond (Dunke1Schetter, 1984; Wortman \& Dunkel-Schetter, 1979). Thus, "The individual may need to adjust to having fewer interactions with fewer people and to receiving decreased confirmation of being a capable individual. The ill for disabled) person must also strive to preserve relationships 
with those friends and family members who satisfy physical and emoticnal dependency needs..." (Miller, 1983, p. 21). In summary, the implications in the chronic disease literature are that (1) individual and family social networks are likely to be seriously disrupted by illness and disability: and (2) continuing social support is crucial to both the person with a chronic disease or disability and his or her family.

One particularly devastating chronic disease which exemplifies the medical and psychosocial aspects of disability, and in which the contributions of social support to recovery and adaptation have not been systematically studied is cerebrovascular accident, or stroke. For both pragmatic and theoretical reasons, this investigation has chosen to focus on stroke as both a health crisis event and chronic disability requiring adjustment and adaptation. These reasons are summarized as follows:

(1) stroke is a ubiquitous event. It is the third leading cause of both death and disability among middle-aged and older persons in this country. An adequate sample of persons who have recently suffered a first, completed stroke would be obtainable within a reasonable period of time.

(2) A stroke is more easily and less ambiguously diagnosed than many other chronic diseases, and has a specific time referent with regard to its occurrence. Being able to specify the onset of the disease or condition will 
be especially important in a longitudinal study assessing social support and adaptation over time. With many other chronic conditions (diabetes, kidney disease, COPD, cancer) onset of the disease is much more difficult to specify.

(3) Strokes are unpredictable and uncontrollable events in terms of specific onset, and usually occur with little or no recognized warning, despite the existence of predisposing risk iactors. Thus, it is less likely that social support and well-being changes observed over time will be confounded by anticipatory changes. Stroke is also more likely, because of its unpredictable and uncontrollable nature, to result in substantial negative impacts on the patient and the family (Rrantz \& Schulz, 1980).

(4) Stroke is not a disease in which a progressive deterioration in the physical condition of the victim is necessarily expected or predicted over the short term, which is the case with many other chronic diseases, such as cancer and lung disease. To the contrary, with recovery and rehabilitation, the stroke patient who has survived the first few weeks after a stroke is expected to show gradual improvement in functioning over the first months to a year, and to survive with the residuals of the stroke for a number of years. This reduces the likelihood that deterioration in physical health status will be confounded with changes in social support and well-being observed following a stroke. The remaining sections of this chapter will review the 
literature on the epidemiology and demographics of stroke, on the physical and psychosocial impacts or stroke on both the stroke survivor and caregivers, and on the impact of social support on the recovery from and adaptation to physical illness, including stroke.

\section{LITERATURE REVIEW}

Definition, Epidemiology and Demographics of Stroke

Stroke is one of the most frequently occuring acute health crisis events afflicting older persons. A generally accepted definition of the term stroke is that formulated by the National Survey of stroke (Weinfeld, 1981):

Stroke is a clinical syndrome consisting of a constellation of neurological findings, sudden or rapid in onset, which persists for more than 24 hours, and whose vascular origins are limited to:

1. Thrombotic or embolic occlusion of a cerebral artery resulting in infarction, or

2. Spontaneous rupture of a vessel resulting in intra-cerebral or subarachnoid hemorrhage.

This definition excludes occlusion or rupture due to traumatic, neoplastic, or infectious processes which produce vascular pathology (p. I13).

Stroke, then, involves a neurologic deficit of relatively rapid onset that is produced by one of several conditions that impairs circulation to a part of the brain. There can be a wide variation in symptoms of stroke affecting motor, sensory, cognitive, language and other functions depending on the areas inrolved in the circulatory 
deficit. The majority of strokes (approximately 80\%) involve infarcts, or the blockage of blood flow to the cerebral hemispheres.

Estimates of the annual incidence of all types of strokes in this country range from approximately 600,000 (Robins \& Baum, 1981) to 750,000 (Rubenstein \& Federman, 1982). Annual incidence of initial strokes is 300,000 (Robins \& Baum, 1981). Prevalence studies indicate that approximately 2.7 million Americans age 20 and over have had at least one stroke when both hospltalized and nonhospitalized persons are included. The national prevalence rate is about 1.6 million if only persons who were hospitalized for the stroke are included (Baum, 1982; Ruller, 1978).

Stroke is largely a disease of older persons. Fiftyseven per cent, or 1.5 million stroke survivors are age 65 and older. Eighty per cent of the new strokes which occur in this country each year affect persons over age 65 (Freese, 1980; Stallones, Dyken, Fang, Heyman, Seltser, \& Stamler, 1972). A recent National Health Survey of a community sample estimated that approximately $80 \%$ of persons who suffer a first stroke are 45 years old or older 1 Baum, 1982). The incidence of strokes of all types, including brain infarction and cerebral hemorrhage, increases sharply with Increasing age (Freese, 1980; Robins \& Baum, 1981). The median age for first strokes in men in this country is 
71 years, and for women is 7 years (Weinfeld, 1981).

stroke is the third leading cause not only oi mortality in older persons, but also of chronic long-term disability. About $60 \%$ of stroke victims survive the acute event, and $40 \%$ of the $1.6-2.7$ million Americans who are stroke survivors require some type of special services or assistance (Freese, 1980). In his analysis of the results of a community survey of a national stroke sample, Baum (1982) (:stimated that $70 \%$ of stroke victims were somewhat limited in their activities, and more than two-thirds were limited in their major activities. Results from the Framingham study of stroke survivors indicated that $31 \%$ were dependent in self care, $20 \%$ were dependent in mobility, $71 \%$ had decreased vocational function, $62 \%$ reported decreased socialization outside the home, and $16 \%$ yere institutionalized when evaluated a minimum of six months after the onset of a stroke (Gresham, Fitzpatrick, Wolf, McNamara, Kannel, \& Dawber, 1975).

While the annual incidence of stroke has been declining steadily in recent years in the United states as well as in other industrialized nations (Garraway, Whisnant, \& Drury, 1983; Soltero, Liu, Cooper, Stamler, \& Garside 1978), survival rates have been increasing (Garraway, Whisnant, Kurland, O'Fallon, 1979). This suggests that the decline in stroke incidence has not resulted in a comparable decline in the number of disabled stroke 
survivors and their families requiring support and assistance. In fact, the prevalence of stroke in the population has remained constant over the past several decades because of major improvement in long-term survival following cerebral infarction (Garraway, 1985). In the Framingham stroke study, women had a five year survival rate of 60\%, while men had a five year survival rate of $52 \%$. The ten year survival rate in this study overall was $35 \%$ (Sacco, Wolf, Kannel, \& McNamara, 1982). In recent longterm follow-up studies of stroke patients who had participated in rehabilitation, the mean survival time exceeded seven years (Anderson, Anderson, \& Rottke, 1977; Anderson \& Rottke, 1978). Thus, while a substantial percentage of stroke sufferers (approximately 30\%) do not survive the first 30 days (Baum \& Robins, 1981), a large number of stroke patients will survive many years with chronic physical and psychosocial disabilities. Furthermore, a very high percentage of these people will be cared for at home or in the community, primarily by spouses and other relatives (Ahlsio, Britton, Murray, \& Theorell, 1984; DeJong \& Branch, 1982; Holbrook, 1982).

\section{Impacts of Stroke on Patients and Careqivers}

In addition to its acute, life-threatening aspects, the occurrence of a stroke is often followed by significant negative life changes for the victim, including role loss, relocation to sheltered care settings, and increased 
dependency on others because of physical and cognitive disabilities. Furthermore, a cerebrovascular accident frequently disturbs an individual's ability to communicate and to interact socially in other ways with family and friends. Equally as important will be the impacts of the stroke on the primary caregiver and other members of the family. Buck (1968) has correctly characterized stroke as a "family disease".

The available literature on the impacts of stroke on the patient and family is largely descriptive, not often data-based, and almost always lacking in comparison or control groups. Nevertheless, the existing reports and studies do suggest a strong relationship between stroke outcome (as measured by adaptation, physical and psychological well-being, and functional independence) and a variety of family interactional and psychosocial variables. This points to the importance of exploring the impacts of this chronic, disabling illness on both victim and family members.

Clinical Descriptions and Reviews. Clinical reports and review articles on the sequelae of brain damage describe cognitive, behavioral, and personality changes which may accompany stroke (Binder, 1983; Gordon \& Diller, 1983; Horenstein, 1970; Lezak, 1978a, 1978b; Stein, Hier, \& Caplan, 1985). Common emotional problems seen in stroke patients include emotional distress, especially anxiety; 
frustration; and depression (Binder, 1983). Estimates of the prevalence of clinicaliy significant levels of depression after stroke range from $26 \%$ to $60 \%$ (Feibel \& Springer, 1982; Robinson \& Szetela, 1981). Robinson \& his colleagues (Robinson, Bolduc, Kubos, Starr, \& Price, 1985), who evaluated 50 stroke patients in the hospital and at six months after discharge, found that in-hospital depression as well as physical and intellectual impairments preaicted social functioning at six month follow-up. other common problems observed in stroke survivors include: overdependence on others, inflexible and rigid thinking, impatience, irritability, impulsivity, denial and lack of awareness of problems, insensitivity, poor social perception, perplexity, distractibility, and fatigue (Binder, 1983, Lezak, 1978b; Stein et al., 1985). Lezak (1978a) cites five broad and somewhat overlapping categories of characterological alterations secondary to brain injury that are most likely to create adjustment problems for families: (1) impaired capacity for social perceptiveness manifested as self-centeredness, and diminished empathy and self-reflective or self-critical attitudes; (2) impaired capacity for control and self-regulation, resulting in impulsivity, random restlessness, impatience, and conceptual and behavioral rigidity; (3) stimulus-bound behavior, reflected as social dependency, difficulty in planning and organizing activities or projects, decreased or absent 
behavioral initiative, and rigidity; (4) emotional alterations manifested as apatiny, siliness, lability, irritability, and changes in the sex drive; and (5) inability to profit from experience, leading to lessened capacity for social learning. Even mildly impaired stroke patients will complain of what Lezak (1978b) has called "subtle sequelae of brain damage": perplexity, distractibility, and fatigue. While not all persons who suffer a stroke and their families will have to deal with all of these behavioral and cognitive changes, it is readily apparent that most of this list has the potential to cause serious disruption in social interaction between the stroke victim and significant others.

While some behavioral and cognitive changes may be characteristic of brain damage per se, other changes are related to the site of the lesion, specifically to whether the stroke affects the right or left cerebral hemisphere. Right-hemisphere brain damage has been associated with lack of concern and awareness of stroke-related deficits, reduced perception and recognition of the affective and emotional aspects of communication, less spontaneity, increased latency of action, and difficulty in persevering with a task, in addition to visual-spatial and constructional deficits (stein et al., 1985). Patients with left hemisphere damage, on the other hand, are more likely to experience significant speech and language deficits because 
the left cerebral hemisphere plays a crucial role in verbal and written communication. While the efrects of a left hemisphere stroke on interactions with family and friends may appear to be more critical, some persons with relatively small right hemisphere lesions do not make the expected social, family, and work-related adaptations following a stroke.

Data-based studies have tended to confirm the clinical reports and reviews of the impacts of stroke. Some studies have focused only on the stroke victims, others only on the principal caregivers, while others have studied the impacts of stroke on both. Taken as a whole, these studies suggest that, while the problems related to physical disability and physical changes may be considerable, disruptions in the social lives of stroke families cannot be explained on the basis of the severity of the stroke or of the physical disability alone.

Studies focusing on stroke Victims. Labi, Phillips, and Gresham (1980) who studied long-term survivors of stroke in the Framingham Study, found that a significant proportion of the stroke survivors manifested psychosocial disability despite complete, or near complete physical recovery. Specifically, three parameters of social function were analyzed to determine the degree of social reintegration of these long-term survivors of stroke: socialization in the home, socialization outside of the home, and hobbies and 
interests. Labi and her colleagues found that in a group of those who could ambulate independently, or who had not experienced any change in their mode of transportation, nor taken any falls, about half decreased their socialization outside the home after the stroke. Further, there was no significant difference found in social activities between those who had fallen during the previous year and those who had not. About one-third of the subjects who had resumed most or all of their household responsibilities did not resume their pre-stroke social activities. Of those with no residual neurologic deficits (hemiparesis, hemisensory defect, hemianopsia, dysarthria, aphasia), $40 \%$ had decreased their socialization outside the home, $32 \%$ had decreased their socialization in the home, and $25 \%$ had decreased their participation in hobbies and interests. The authors concluded that "much of this social disability cannot be accounted for by age, physical impairment, or specific neurologic deficits" (Labi et al., 1980, p. 561). A strength of this investigation was the use of an age-matched control group of non-stroke victim study participants.

A possible explanation for the results of the Framingham study is what Hyman has called the "stigma of stroke" (Hyman, 1971). In studying the rehabilitation motivation and the amount of functional improvement in a sample of 110 stroke patients with hemiplegia, Hyman hypothesized that "Feelings of stigma may render the 
prospect of social interaction unrewarding to the patient. He would therefore become conditioned to minimizing his social contact..." (Hyman, 1971, p. 133). Hyman operationally defined feelings of stigma in patients as the belief "that others think less of them, avoid them, or $f \in a l$ uneasy with them because of their 1llness or disability" (Hyman, 1971, p. 132). He further speculated that patients who reported feelings of stigma at the beginning of a rehabilitation program would be rated by their physical therapist and physician as less motivated after one month of therapy. Hyman found that feelings of stigma manifested at the start of the program adversely affected motivation at one month as well as functional status at discharge, despite the fact that both the stigmatized and non-stigmatized patients had been comparable on levels of functional ability and on other medical variables at the beginning of the program. Patients who felt stigmatized at the start of rehabilitation were less likely to resume household tasks performed prior to discharge. He also found that of the patients without feelings of being stigmatized, $73 \%$ resumed all premorbid leisure activities, while of the stigmatized patients, only 45\% had resumed all premorbid leisure activities. Interestingly, these negative relationships between degree of stigma and level of motivation and ADL independence were limited to those patients who pre-morbidly were more socially active. Hyman also, and perhaps more 
importantly, found a non-significant but suggestive association between the patients' feelings of stigma and their relatives' perception of them as stigmatized. This study suggests important connections between individual reactions to chronic impairment and the social network's reactions to the individual.

other recent descriptive studies of stroke outcome have tended to confirm that psychosocial impairments and distress in stroke survivors cannot be explained by the level of physical disability alone. Isacs and his colleagues (Isaacs, Neville, \& Rushford, 1976; Isaacs, 1982) followed for three years or until they died, a group of 29 stroke survivors who were discharged home. Of the 18 who were still living after three years, none had returned to employment, and few engaged in any activities outside the home. A Swedish study (Ahlsio et al., 1984) followed 96 recent stroke patients for two years, completing interviews with 50 of the subjects at all four interview times lat discharge, and at one month, six months, and two years postdischarge). Of the survivors at two years, $76 \%$ were independent in activities of daily living (ADL's) and lived at home. The researchers had subjects rate their quality of life before and after the stroke using a visual analogue scale. Results indicated that most stroke victims had experienced a decrease in their subjective quality of life, and that no improvement in this aspect of their lives was 
observed over the two year period. Although ADL function improved during follow-up, quality of iife did not. Depression and anxiety were found to be equally as important as ADL status in predicting quality of life. Furthermore, depression or anxiety in the acute phase of the illness was predictive of greater decrease in quality of life at two years.

Lawrence and Christie (1979) found evidence of poor adaptation to their post-stroke situation in 45 persons who had suffered a stroke three years previously. They classified subjects as having "minimal" or "moderate" levels of physical disability, having no survivors in their sample with severe residual physical disability. They classified reactions to disability and to the stroke victim's situation as appropriate or inappropriate. An appropriate response was defined as either minimal disruption in the person's pre-stroke life situation, or realistic response to disability and lifestyle changes. Inappropriate responses were categorized as those reflecting denial or exaggeration of problems, little adaptation to stroke-related changes, or frank behavioral disturbance. Over one half of these stroke survivors were judged to have an inappropriate response to their situation, including $44 \%$ of those with minimal impairment. Twenty-two of the subjects exhibited either denial or exaggeration of their problems, with iittle or no constructive effort having been made to adapt to 
difficulties, while two were felt to show gross behavioral disturbance with "Iittie grasp of reality". These researchers found no significant relationship between the degree of disability and the appropriateness of the adaptive response. Though they did find a significant association between level of disability and degree of optimism about the future, over half of the subjects with minimal disability were uncertain or pessimistic regarding the future. Other negative changes experienced by these stroke survivors in the three year period included a marked change in occupational status, significant decreases in active leisure activities, and deterioration of interpersonal relationships within the household in one-third of those with minimal disability and two-thirds of those with moderate physical impairment.

In another study which examined quality of life issues, Trudel, Fabia, and Bouchard (1984) interviewed fifty persons who had undergone carotid endarterectomy from 81 to 105 months prior to the investigation. Some of these persons had experienced completed strokes, that is, strokerelated neurological symptoms which persisted for longer than 24 hours; however, all subjects were described as having minimal dysfunction in ADL's and cognition. Subjects were retrospectively classified into low or medium preoperative risk groups based on other medical problems and severity of cerebrovascular disease as determined by 
angiography. Those in the medium risk group were found to have marked dysfunction in home and outside activities and social interaction and half of those previously working had taken early retirement. Quality of life, the authors concluded, appeared to be more affected by cardiovascular problems in the medium risk group, and by neurologic problems in the low-risk patients. Overall, only 10 of the 50 subjects were found to have a normal functional level. We thus see that even in a population with minimal impairment, and who underwent carotid artery surgery to prevent the occurrence of a debilitating stroke, psychosocial outcomes were, on the whole, poor.

In summary, the studies of the impacts of stroke on the patients themselves lead to the conclusion that psychological and social factors including interpretation of the stroke event and social support may be more important than physical status or initial level of disability in determining outcomes.

Impacts of stroke on Careaivers and Families. Several studies included information obtained from both stroke patients and families. These studies also paint a picture of poor adjustment by stroke victims and multiple stresses experienced by families. Belcher, Clowers, and Cabanayan (1978) interviewed 73 post-stroke individuals with a mean age of 60 years. Forty -two were married, and 31 single. The spouses of the married stroke victims were also 
interviewed. The focus of this study was on the current rehabilitation needs of this population. While vocational needs were more important to the single stroke survivors, social psychological needs were found to be more important to the married stroke victims and their spouses. The authors concluded that while many post-discharge stroke patients express the need for more physical rehabilitation, a more predominant concern is psychosocial rehabilitation. Neither levels of distress nor other symptoms of psychological dysfunction were measured in this study.

In a three year follow-up study of 32 stroke families, Cohen (1978) found that two-thirds of the 21 survivors were ambulatory and continent at the end of the three year period. All 32 had been discharged to live in the community - half home to their own immediate family, 4 to live with children, and 12 to live alone in the community with some assistance. These relatively independent stroke survivors were found at three years to be gloomy, reserved, and withdrawn, with little effort shown to maintain social contacts. Significant personality changes were observed which the author attributed to the effects of brain damage and to changes in patterns of living imposed by restricted opportunities for socializing. Families reported such problems as irritable, combative, and uncooperative behavior; marked depression; incontinence; and loss of appetite. Before their CVA's 18 of 32 had been employed full time and 
7 were performing housekeeping activities with no problem. After the stroise, none returned to gainful employment, and only 3 were able to resume some housekeeping activities. More than half suffered a substantial decrease in their economic well-being. About half of those who survived the first year did not leave the house, while the others got out only occasionally.

Holbrook (1982) reported data from two follow-up studies of a total of 180 stroke patients and families interviewed 2 to 5 years after the stroke. She found that stroke patients and their caregivers reported different concerns and different negative impacts of the stroke. stroke survivors reported that the stroke had adversely affected their relationship with the family, their relationship with their spouse, and their sex life. Specific concerns centered around mobility, health, and finances. For the caregivers, adverse effects of the stroke were related to finances, social mobility and social life, interpersonal relationships, sex life, working life, and physical health. One-third of both stroke victims and caregivers described themselves as "not adjusted" to the stroke 2 to 5 years afterward. The investigators noted that adjustment did not necessarily occur if the residual physical disability was slight, and that stroke victim adjustment did not necessarily predict caregiver adjustment. A younger sample of stroke survivors and their spouses 
was studied by Coughlan and Humphrey (1982). They interviewed by means ố a mail questionnaire, 170 spouses of stroke patients who had received rehabilitation up to 8 years earlier. Information was obtained from these spouses about both the stroke victim's status and their own experience regarding the stroke. All stroke victims were less than 65 years old at the time of the CVA, and $40 \%$ were under 50. At 3 to 8 years after the stroke, two-thirds of the stroke survivors were described as having some problems with self-care while almost half had restricted mobility. of the total group, one-fourth had marked personality change, while two-thirds had some personality change, although this did not correlate with either side of lesion or the presence of hemiplegia. One-fourth of these stroke victims had experienced communication loss, while one-third had reported memory loss. Of the male stroke victims who were under 65 years of age at the time of follow-up, 30\% were in paid employment, and 5 others had worked at some time following the stroke. of the female stroke victims, 17\% of those under 60 were gainfully employed, compared to 58\% of those under 60 at the time of the stroke who were employed. Personality changes in stroke victims reported by their spouses included irritability, loss of self-control, impatience, decreased frustration tolerance, emotional lability, self-centeredness, and decreased initiative. Spouses of stroke victims rated the stroke survivors' 
enjoyment of life since the stroke as "much less" in $41 \%$ of cases, compared to enjoyment of life before the stroke. They attributed their spouses' loss of enjoyment to residual disabilities, loss of independence, and lack of occupational interests. For the caregivers, occupational changes and decreased life satisfaction had also been experienced. Five male and 18 female caregivers reported not working since their spouse's stroke. Thirty-two per cent of the caregiver spouses reported that their enjoyment of life was "much less". While the spouses of hemiplegic stroke victims were more likely to report decreased enjoyment of life regardless of side of hemiplegia, they were significantly more likely to report a loss of enjoyment of life if the stroke victim had suffered a right hemisphere stroke with left hemiplegia than if the stroke victim was non-hemiplegic. Spouses attributed their decreased enjoyment of life since the stroke to loss of companionship, increased domestic responsibilities, and interference with leisure and social activities.

To summarize, studies which have considered the outcome of stroke for patients and caregivers together indicate some differential impacts of the stroke depending on whether one is the patient or caregiver. They also point to the possible interactive effects of characteristics of the stroke, patient personality variables, and caregiver well-being. 
Studies Focused on Caregivers. Several studies reviewed focused only on the adjustment and well-being of the primary caregivers of stroke survivors. Some of these investigations were concerned with strokes in general, some focused on the caregivers of stroke victims with significant communication deficits, while others compared the outcomes for spouses of patients with and without the language deficit of aphasia. These studies will now be briefly summarized.

Mackay and Nias (1979) interviewed the caregivers of 28 younger (under age 65 at the time of the stroke) incapacitated stroke victims who required substantial home care six months after the patients' discharge from the hospital. Focus of the interview was on the caregivers' own social, economic, and emotional problems related to the stroke. These caregivers were all relatives: 19 wives, 3 husbands, 4 daughters, 1 sister, and 1 brother. Eight of 12 who had been working prior to the stroke had had to give up their jobs, 8 caregivers considered themselves worse off financially since the stroke, 25 of 28 had to now spend most or all of their time at home, and eight had taken tranquilizing medication since the CVA. Yet 20 of 28 caregivers were judged by the interviewer to be "moderately happy and confidant". The remaining eight of the 28 caregivers reported feeling "very depressed" and were described as struggling to cope. Only a small number 
reported feeling that their relationship with the stroke victim had deteriorated (3 of 28). Unks (1983), who interviewed 50 elderly wives of chronic stroke patients, also found relatively high levels of morale in these caregivers. He utilized the Philadelphia Geriatric Center Morale scale as the outcome measure. These wives had morale scores which were comparable to or only slightly below those of the general elderly population.

Brocklehurst and his colleagues (Brocklehurst, Morris, Andrews, Richards, \& Laycock, 1981) interviewed the primary caregivers of 97 new stroke patients between 4 and 6 weeks after the stroke and at "regular intervals" for one year. Reported deterioration in the caregiving person's health was common during the first year, tripling from the first interview to the one year post-stroke interview. Over 25\% of the caregivers had responsibility for other persons in addition to the stroke victim. Major problems cited by the caregivers were related to the behavior of the stroke victim, the need for constant supervision, and loss of sleep. Although there had been a large amount of assistance offered to the caregiver by relatives, friends, and neighbors during the early post-stroke period, little assistance was being received by the primary caring person at the end of a year. Fourteen per cent of this caregiver sample had given up their jobs because of the stroke. It should be noted that this was quite a dependent stroke 
sample - 55\% were dependent for everything but personal care, and 25\% were totaily dependent.

Silliman (1984) interviewed 89 caregivers of elderly stroke patients, 82\% of whom returned home following hospitalization. Silliman compared the changes experienced by those persons who were caring for their family member at home with those whose relative had been discharged to a nursing home (potential caregivers). She found that caregivers reported both good and bad effects of the stroke on their lives. Eighty-four per cent of home caregivers said that they felt better about themselves because they had learned to manage their relative's illness, and 69\% reported closer relationships with the patients because of the illness and caregiving experience. On the other hand, 75\% worried about the consequences of caregiving on their own health, $40 \%$ to $45 \%$ attended church less often, had less time for other members of their families, noted more financial burdens, and had less time for themselves. In addition, $40 \%$ scored high on the General Health Questionnaire, an instrument designed to screen for symptoms of emotional ill health. This is twice the prevalence reported in community surveys. Unexpectedly, silliman found no differences on the measures of emotional health and adjustment between those actually caring for stroke-victim relatives and those whose relatives were in nursing homes.

Three studies compared the impacts on the caregiver of 
stroke resulting in left hemisphere brain damage and aphasia with stroke without the presence of aphasia lartes and Hoops, 1976; Fengler and Goodrich, 1979; Rinsella and Duffy, 1978, 1979, 1980). Artes administered a 263-item questionnaire to 65 wives of stroke victims whose husbands were residing at home. Thirty-five of the stroke victims were aphasic, while 30 were noted to have no communication impairment. The wives of the aphasic individuals rated all problem areas explored (health and physical care, economic changes, communication behavior, and psychosocial aspects of behavior) as of more concern to them than did the nonaphasic victims' spouses. Interestingly, however, less than half of the wives of aphasic persons considered the communication impairment itself to be the major problem with which they were faced. They reported less opportunity for social activity (43\%), visitors calling once a week or less often (49\%), and that the aphasic spouse was "hard to get along with" post-stroke (46\%). In contrast, only $17 \%$ of the non-aphasics' spouses reported less opportunity for social activity, $33 \%$ reported visitors calling once a week or less often, and $33 \%$ reported that their stroke-victim spouses were difficult to get along with since the stroke. Almost $30 \%$ of the wives of aphasic victims reported that they never got away from the house by themselves compared to $13 \%$ of the non-aphasic group's spouses. Forty-four per cent of the wives of the non-aphasic stroke victims reported a $10 \%$ or 
less curtailment in their social activities, while only $12 \%$ of the aphasic individuals' wives experienced that low a level of diminished social activity.

Rinsella and Duffy (1979) also found more severe poststroke adjustment problems in the spouses $(70 \%$ female) of aphasic persons compared to the partners of non-aphasic stroke survivors. Spouses of aphasic, hemiplegic persons were found to have significantly poorer overall adjustment, to be significantly more lonely and bored, and to be more maladjusted in their marital relationships. These investigators found that spouse adjustment was not significantly related to the stroke victim's dependency, severity of hemiplegia, or the severity of the victim's aphasic impairment. More disturbing, the greater the time between the onset of the stroke and time of interview, the significantly worse the adjustment score. This was a crosssectional study, however. Similar results comparing the interpersonal situations of spouses of aphasic and nonaphasic stroke survivors were found by Fengler and Goodrich (1979).

Common themes characterize descriptive reports of the stresses and concerns of families of aphasic stroke survivors. These anecdotal accounts have documented the disruptions which occur in interpersonal interactions when a family member suffers from aphasia. Webster (1980; Webster \& Newhoff, 1981) described the most commonly reported 
problems of wives of aphasic stroke victims: problems relating to the assumption of many of the duties formerly performed by the spouse; lack of time for themselves; lack of companionship; lack of people with whom to talk. These women reported that they could not discuss their feelings of isolation with their families or neighbors for "fear of stirring guilt in family members, or appearing to neighbors as bad wives" (Webster, 1980, p. 351). Malone (1969) interviewed 25 persons representing the families of 20 aphasic adults, including spouses, children, and other relatives. He found that in every case, families reported that the family as a closely knit unit no longer existed. Role changes were one of the most frequently mentioned problems. Nearly all families stated that their friends gradually stopped coming to visit. Several of the families reported health problems in the non-aphasic family members since the stroke, which left them less able to cope. Malone also found that spouses frequently mentioned the negative effects of having an aphasic family member on the social development of the children.

In the only published study found dealing especially with the impacts of aphasia on the lives of children of aphasic individuals, Chwat, Chapey, Gurland, and Pieras (1980) administered a 50-item questionnaire to 16 children of 16 aphasic adults. The children ranged in age from 16 to 41 years, with a mean age of 26 years, and all were reported 
to be in face-to-face contact with their parent at least three times weekly. Almost $70 \%$ of the respondents reported playing the role of parent, bearing a greater share of the responsibility for household tasks and other necessary chores than siblings, at least part of the time. Half reported that they lost patience with their aphasic parent and felt irritable after spending time with them, while more than one-third indicated that making visits to their parent was anxiety provoking. Two-thirds of the children felt that they interacted with their communicatively impaired parent an inappropriate amount of time (either too much, or too little, about equally divided). Almost $40 \%$ of respondents felt that their social lives had changed considerably since the stroke and that care of their parent frequently took time away from their own social activities.

In summary, what can be said about these descriptive and correlational accounts of the impacts of stroke? There are many problems with these studies as a group. Small sample sizes, questionable validity of some of the outcome measures, and absence of control groups limits their interpretation and generalizability. Few studies presented any data regarding the pre-stroke status of individual and family social networks, except for some retrospective comparisons. Nevertheless, they consistently portray a picture of psychological, social, and economic distress for both stroke victims and caregivers. The question arises as 
to whether persons who suffer a stroke are unique in the negative outcomes they experience from a chronic illness or disability. The next section of this chapter will briefly explore this question.

\section{Impacts of Chronic Illness in Other Populations}

Individuals' adjustments to a variety of chronic diseases including heart disease, cancer, epilepsy, lung disease, and kidney disease have been studied. The way people cope with chronic disabilities has also been investigated. This research has yielded somewhat mixed results which, taken as a whole, do not reflect a consistently negative picture of adjustment to health stresses. Cassileth and colleagues (Cassileth et al., 1984) studied 758 patients each of whom had one of six different chronic illnesses: arthritis, diabetes, cancer, renal disease, skin disorder, and depression. They found that the patients with physical illnesses did not difier significantly from one another or from the general public in terms of their scores on a mental health index. Patients in the five physical illness groups did, however, have significantly higher scores for psychological status than the patients being treated for depression. The authors concluded that these chronically ill people showed remarkable psychological adaptation.

A different picture emerged from a study by Noh, Wood, and Turner (1984). These authors explored the prevalence of 
depression among a population of people with a variety of unspecified physical disabilities, and varying degrees of impairment. The subjects ranged in age from 18 to 91 years, with a mean age of 56 years and a mean duration of disability of 16 years. In this sample of 1005 persons, 46\% were male, and virtually all were caucasian. The depression measure used was the Center for Epidemiologic Studies Depression Scale (CES-D) (Radloff, 1977). What Noh and his co-workers found was that the physically disabled were twice as likely as the general population to score in the risk group for clinically significant depression on the CES-D. Further, their results showed that the elevated scores observed in the disabled population could not be attributed to inflated scores on somatic items nor to a disproportionete number of transient symptoms. They found the disabled group to be at an importantly elevated risk for clinically significant depression across all sex, age, residence, and marital status categories.

In a study involving a more homogeneous sample of disabled persons, older spinal-cord injured men, schulz and Decker (1985) obtained a somewhat different result. They used three standardized instruments to measure adjustment: Index of Psychological Well-Being, Life Satisfaction Index, and CES-D. For all three outcome measures, schulz and Decker found that these men, who were all several years out from the onset of their disability, reported levels of well- 
being only slightly lower than population means of similarage nondisabled persons.

One group of patients whose post-illness adjustment has been extensively studied is cardiac patients. Researchers who have looked at the psychological status of heart attack patients during the acute hospitalization phase have generally observed high levels of stress in these individuals (Cay, Vetter, Philip, \& Dugard, 1972; Cassem \& Hackett,1971; Doehrman, 1977). Doehrman (1977), in his review concluded that studies which have examined psychological reactions of cardiac patients in the posthospital period provide evidence of "considerable anxiety and depression which persists months and sometimes years" (p. 206). Yet other studies have found relatively good adjustment in patients with chronic heart disease (Brown, Rawlinson, \& Harden, 1982; Croog \& Levine, 1982; Croog, Lipson, \& Levine, 1972). Brown and her colleagues examined the functioning of 48 families of heart patients at 9 months and more post-onset. They found these families to be functioning quite well, as judged by scores on the Family Functioning Index (Pless \& Satterwhite, 1973). They did find, however, that more anxious patients (as measured by the Anxiety scale of the MMI) had lower family functioning scores. Croog and Levine (1982) found generally positive results in their longitudinal study of caucasian men 8 years after a heart attack. In their sample, $86 \%$ reported being 
satisfied or very satisfied with their lives, 918 of those eligible for employment were employed, $75 \%$ reported low or medium levels of stress, and $76 \%$ had no depressive reaction to their illness. Even many of the recently hospitalized men reported the overall quality of their lives as favorable (74\%). One possible explanation for the differences in psychological adjustment reported in studies of heart patients is that a sudden, unexpected heart attack in persons previously considered healthy may have quite different impacts than heart disease of gradual and insidious onset without the dramatic and life-threatening implications of a heart attack.

Two other studies which explored the adjustment of persons living with chronically altered life styles related to physical illness produced somewhat contradictory results. In a study of long-term colostomy patients, Wirsching, Druner, and Herrmann (1975) found that active contact with friends was diminished significantly after surgery, as were other social activities outside the home. significantly higher rates of loneliness and depression were reported for the colostomized group compared to a control group of persons who had also undergone colon surgery for cancer but had not required a colostomy. In contrast, in studying a small group of patients on continuous ambulatory peritoneal dialysis (CAPD), in which the authors acknowledge there may have been some selection bias, Maida, Wolcott, Ratz, and 
Nissenson (1983) measured perceived control over factors affecting health status (health locus of control), coping ability, family relationships, social support, and anxiety/affect. Their results suggested that overall this sample of CAPD patients adapted well to the stresses of their kidney disease and to the treatment modality.

In sum, there is evidence to suggest that persons with other chronic illnesses and physical disabilities may cope better with their situations than do stroke patients. This may be due to the combination of cognitive and observable physical changes which are associated with a disabling stroke. The evidence is less equivocal and more consistent in terms of the impacts of chronic illness on caregivers, however. The studies which have examined the psychological and physical well-being of caregivers of persons with a variety of chronic disabling conditions have found high levels of stress and other symptoms in this population (Dhooper, 1984; Fengler \& Goodrich, 1979; Klein, Dean, \& Bogdonoff, 1967; Sexton, 1984; Gilleard, 1984).

After examining the literature on the impacts of chronic illness on victims and families, an intriguing and important question emerges: Why do some persons and families cope better with not only the initial crisis of illness (Moos \& Tsu, 1977), but with the daily stresses of living with a chronic, disabling condition? In exploring the link between stressful life events (Dohrenwend \& 
Dohrenwend, 1981; Holmes \& Rahe, 1967; Rabkin \& Streuning, 1976; Vinokur \& Salzer, 1975) and haalth outcomes, attempts to document the relationship between life changes and health using a "stressful life events" perspective (Holmes \& Rahe, 1967) have found very small correlations between the number of stressful life changes persons experience and subsequent mental and physical health outcomes (Rabkin \& Streuning, 1976). The concept of "social support" was suggested as an important mediating variable to explain why stress does not consistently result in negative health outcomes (Cassel, 1974; Cobb, 1976; Dean \& Lin, 1977). Subsequently, in the $1970^{\prime} \mathrm{s}$ and $1980^{\prime} \mathrm{s}$, studies of social support have proliferated. Despite lack of consistency and comparability in definitions of social support, and methodological and research design weaknesses in many of the studies (Cohen \& Wills, 1985; Heller, 1979; House \& Rahn, 1985; Thoits, 1982) a substantial body of evidence has accumulated which, taken together, suggests that social support and social connectedness are centrally related to psychological and physical health. Various conceptual definitions of social support, ways of describing social networks and their supportive functions, and a review of findings from some of the representative social support literature will follow in the next section of the chapter. 
Social Support: Definition and Measurement

Descriptive Definitions of Social Supoort. The construct of social support has been described and defined in a number of different ways. Operationalization of the construct in specific research contexts is frequently based on one of the following definitions. Cobb (1976) defined social support in terms of the information available to an individual which leads that person to believe that he/she is 1) cared for and loved; 2) esteemed and valued; and 3) belongs to a network of communication and mutual obligation. Weiss (1974) suggested that social support is a combination of at least six categories of what he called relational provisions: attachment, social integration, opportunity for nurturance, reassurance of worth, sense of reliable alliance, and the obtaining of guidance. Caplan (1974) defined social support by emphasizing that social support systems refer to "enduring personal ties to people who can be relied on to provide emotional support, help, and reassurance in times of need; they also provide feedback, and share common values and goals." Walker, McBride, and Vachon (1977) provided a functional definition of social support: "...that set of personal contacts through which the individual maintuins his sonial identity, and receives emotional support, material aid, services, information, and new social contacts." Kahn and Antonucci (1980) proposed that social support be defined as "interpersonal 
transactions that include one or more of the following key elements: affect, affirmation, and aid" (p. 257). In summarizing broad categories of social network functions described in the literature, Mitchell and Triskett (1980) listed emotional support; task-oriented assistance; communication of expectations, evaluation, and a shared world view; and access to new and diverse information and social contacts.

Network Analytic Approaches. In contrast to these definitions of the supportive functions of social networks, other authors have advocated adopting an approach based on social network methodology (Wellman, 1981; Hall \& Wellman, 1985). This approach, rather than defining personal social networks as supportive in an a priori fashion, describes the social network in terms of its structural and relational characteristics. Information about the content of network exchanges and other aspects of the network can be utilized to describe and explain the flow of needed resources to and from the focal individual (Wellman, 1981). Definitions of social networks which incorporate a network analytic perspective make no assumptions about the supportiveness of the network. For example, Mitchell (1969) defined social networks as a "specific set of persons, with the property that the characteristics of those linkages as a whole may be used to interpret the social behavior of the persons involved." Bott (1957), another network researcher, defined 
social networks as "all or some of the social units (individuals or groups) with whom a particular individual or group is in contact." Hall and Wellman (1985) describe networks as simply "a set of nodes that are tied by one or more specific types of relations between them". It is obvious that, while these definitions do not carry the connotation that all network relationships are supportive, an individual's social support system will be found within his or her network of social relationships. Network analysts would argue that the terms "social support" and "social network" should not be confused or used interchangeably: "While a support system is a social network (i.e., a set of nodes (persons) and a set of ties between nodes, it is an analytically constricted network which only takes into account supportive ties" (Wellman, 1981, p. 173). Social support researchers have more recently begun to consider the possible negative impacts of network ties (Dunkel-shetter, 1984; Abbey, Abramis, \& Caplan, 1985) with results that have increased our understanding of social support processes.

Several structural and interactional characteristics of social networks which have been shown in at least some studies to have relevance for social support are listed and defined in Table $I$. Illustrative studies are those of Hirsch (1980) and Wilcox (1981), who found that for persons facing major life charges which required adjusting to new 
TABLE I

STRUCTURAL AND INTERACTIONAL SOCIAL METWORR CUARACTERISTICS AND THEIR DEFINITIONS

\section{Structural Network Characteristics}

Size or Range. The number of persons with whom the focal person has direct contact, defined by the manner in which network names are elicited in a particular study. Researchers may ask subjects to name the most "important" persons in their network, those to whom they feel "closest", or those who provide specific types of support or aid.

Density. The extent to which members of an individual's social network contact or know each other independently of the focal person. It is defined as the number of actual ties among network members divided by the total number of possible ties.

Degree. Related to network density; the average number of relationships that each member has with other members of the network.

Boundary Density. The degree of overlap in the social networks of two focal persons, for example husband and wife. It is defined as the number or proportion of persons the two networks share in common.

Clustering. Density within specific segments, or subgroups of the network. Patterns of relationships within a network may be identified by examining clusters, or network subsets. 
TABLE I (Continued)

\section{Interactional Characteristics of Network Linkages}

Composition. The relationships which exist between the focal person and other network members (e.g.. spouse, child, other family member, friend, neighbor, work associate). The network can be described in terms of the relative proportions of different types of relationships.

Multiplexity. The number of functions served by a relationship. This is frequently defined in terms of the number of different support functions a particular relationship provides (emotional support, tangible aid, information and advice, etc.)

Frequency. The frequency with which the focal person has contact with members of the network. This may be defined as face-to-face contacts, other types of contacts, or a combination.

Reciprocity. The degree to which support or aid is both given and received by the focal person. It may be defined by counting the number of reciprocal relationships within a personal network, or by asking the focal person to rate the degree of assistance or support given or received in each relationship. In either case, a reciprocity ratio can be calculated.

Durability. The degree of stability of the individual's links with others in the network. Usually measured as the average length of time network members have been known.

Dispersion. The ease with which the focal person can make contact with members of the network. Usually this is measured in terms of geographical proximity.

Homogeneity. This refers to the extent to which members of a network share common social or demographic attributes (e.g., age, ethnicity, religious affiliation, SES, etc.). Not often used in network studies. 
roles (returning to school in mid-life, being widowed at a young age, coping with divorcel, loosely-hnit, less dense networks were related to a greater sense of well-being. These authors speculated that more diverse networks provided access to needed information, advice, and positive feedback for persons experiencing life transitions. Thus, a network analytic approach allows one to describe the composition, structure, and contents of naturally existing social networks. It can provide aggregate information about network composition (for example, the number of kin or friends in a network), as well as structural information about the arrangement of ties (for example, clustering, density) which may help to explain the availability and flow of support resources (Hall \& Wellman, 1985).

Other Measures of Social support. While some researchers have directly studied and measured social support functions (for example, emotional support, instrumental support, informational support), and others have used network analytic approaches to describe structural aspects of the network (Hirsch, 1980; Tolsdorf, 1976; Wilcox, 1981), some have used attribute measures (for example, marital status) or count measures of activity in social and religious organizations as indirect indicators of social support. While previous research (Berkman \& Syme, 1979; house et al., 1982) has shown that it is important to include marital status and level of social integration in 
studies of the effects of support, approaches where only a single measure of status or activity is used as an indicator of social support are no longer considered adequate. In fact, recent comprehensive reviews (Cohen \& Wills, 1985; DiMatteo \& Hays, 1981; House \& Rahn, 1985; Wortman, 1984) have suggested that multiple measures of the structural and relational aspects of the social network, of the variety of supportive functions provided by the network, and of characteristics indicative of the level of individual social integration are necessary. Cohen and wills (1985), for example, have concluded after a careful analysis of the literature which has explored the buffering effects of social support, that both direct effects of supportive ties (that is, having social support available results in better outcomes regardless of the level of stress experienced) and stress-buffering effects of social support (that is, that the positive impacts of support will be greater for those experiencing higher levels of stress) will be found, depending upon whether structural measures of network characteristics or the variety of supportive functions provided by the network are employed as measures of support. Other issues concerning the measurement of social support have recently been emphasized in reviews focusing on the relationship between social support and coping with serious and chronic illness (DiMatteo \& Hays, 1981; Wallston, Alagna, DeVellis \& DeVellis, 1983; Wortman \& 
Conway, 1985). These include: (1) The importance of considering the possible negative aspects of social networks, and the negative impacts of supportive interventions on adaptation to illness; (2) the necessity of considering both actual and perceived social support; and (3) the necessity to consider the interactive nature of the influences of the illness or disability on the social network and of the support system on the adaptation to chronic illness/disability. With regard to the negative aspects of social support, it has been suggested that incongruence between the perceived need for support on the part of the recipient and the provision of support by persons in the network may result in poor adaptation and a level of recovery which is less than expected. Particularly in situations where individuals are coping with serious physical illness or disability, inappropriate types and amounts of support may result in reduced self-esteem, and greater psychological distress for the victim and subsequently for the caregiver (Dunkel-schetter, 1984).

Regarding perceived availability of support, several researchers have suggested that whether an individual believes support to be available should he or she need it is perhaps more important than whether that support is actually tapped (Cohen, McGowan, Fooskas, \& Rose, 1984; Turner, Frankel, \& Levin, 1983). In addition, the individual's perceptions regarding the adequacy of the quantity and 
quality of available support may be more related to outcomes than measures of support usage (Barrera, 1981; Connor, Powers, \& Bultena, 1979; Hirsch, 1980; LaRocco, House, \& French, 1980; Porrit, 1979; Sandler \& Barrera, 1984; Ward, Sherman, \& LaGory, 1984).

Finally, most studies of social support and most conceptualizations of the construct have failed to consider the dynamic nature of personal social networks, and that serious illness and chronic disability can severely stress the support system of an individual and of the family (Eckenrode \& Gore, 1981; Gore, 1981; Wortman \& Conway, 1985). Typically, studies of social support have measured particular aspects of social networks or supportive functions of the network at one point in time, even if the study was longitudinal. Measures of network change and its influence on well-being have not been considered prospectively in studies of recovery from serious illness and adaptation to chronic physical conditions.

In summary, current critiques and reviews of the social support literature, agree that while the weight of the evidence indicates that supportive networks are beneficial, greater specification regarding the types, sources, appropriateness, timing, and perceptions of social support are needed in order to increase understanding of relationships between stress, social support, and health outcomes. Examination of some of the representative 
studies in the social support literature may help clarify what questions regarding these relationships remain to be answered. Because the social support literature is so vast, the following literature review will concentrate (1) on those studies which have focused on recovery from and adaptation to physical illness and chronic disability; and (2) on those studies which concern informal social networks of family, friends, and neighbors rather than those which have investigated the role of the formal support system of health care professionals and others in ameliorating the stresses of illness and disability. Because much of the work in this area has focused on two common and serious illnesses, heart disease and cancer, these will be given more attention. What is known regarding the social support networks of stroke victims and their caregivers will be considered separately as well.

Social Support and Adaptation to Chronic Physical Illness

Social Support and Coping with Heart Disease. Several studies which have examined the role of informal support systems in recovery from heart attack and heart surgery have been purely descriptive in nature. That is, they have described the types and sources of support utilized by heart patients, but have not specifically investigated the relationships between supportive networks and adaptation/recovery. One such study is that by Croog, Lifson, and Levine (1972; Croog Levine, 1982). These 
investigators examined the perceptions of level of support furnished by kin, neighbors, and friends; the level of reported use of formal sources of help and support; and the pattern of types of help furnished by kin, neighbors, and friends (coded as services, moral support, or financial aid) in a sample of 293 previously healthy Caucasian men who had recently experienced a heart attack. At 1 year following discharge, the highest proportion of network members who were perceived as "very helpful" were members of the subject's own family of orientation (siblings and parents). Friends were rated as very helpful almost as frequently as were family, and neighbors were more often considered very helpful than were other relatives. Pertinent to the notion of negative network relationships, substantial proportions of family (25\%), other relatives (almost half), and neighbors (about one-third) were rated as "not helpful at all", while only $16 \%$ of friends were considered not helpful. Patterns of type of support provided depending on relationship to the recipient were also noted. Moral support was reported as being a frequent type of support provided by all relationship groups, especially family and friends. On the other hand, neighbors tended to provide more services, and families were almost the sole providers of financial aid. Reported pre-illness frequency of visits with friends and neighbors was associated with the amount of perceived help from these sources after the heart itssck. 
Patients with illness setbacks (29\%) reported higher levels of help from friends only. Finally, croog and his coworkers found strong associations between and among the degree of helpful support from all sources. They concluded that overall, their findings could best be explained by the pre-illness social integration of the individual, rather than by specific aspects of support provided after the heart attack.

Dhooper (1984) obtained similar results in investigating the adjustment of 40 recent heart attack victims and their spouses. The spouses were actually the subjects of the study. Dhooper operationalized social support as the level of family integration (defined as the sum of social contacts and trichotomized as low, medium, or high); the perceived helpfulness of support received; and the types and sources of support. She found that relatives, especially adult children, were the primary sources of aid, that families judged to be more highly integrated perceived support as more helpful, and that families in higher socioeconomic (SES) groups perceived support as more helpful than those in the low SES group. Results indicated that most support for these families leveled off at about one month after discharge from the hospital, despite the fact that at the one month interview, 90\% of spouses were still experiencing some anxiety and 55\% had not resumed their social activities. 
Finlayson (1976) went beyond description of network resources in her study of 75 women whose husbands had survived for 12 months after a myocardial infarction. She studied several network variables including type of relationship, presence or absence of a confidant, and types of support provided by various sources of support. Outcome, which Finlayson related to network characteristics, was classified as (1) favorable (husband working and wife defined the illness as over); (2) intermediate (husband working, but wife had not defined the illness as over; or (3) unfavorable (husband not working). Results of this study implicated differences in both structural characteristics of the social network and in types of support perceived as available in explaining unfavorable outcomes. Families where the wives acknowledged fewer types of sources of network support, and where support sources were predominantly restricted to the immediate families of both spouses tended to experience less favorable outcomes. Those who lacked a confidant, or "lay consultant" also reported less favorable outcomes. An interesting finding of this study was that SES interacted with outcomes according to the source of support. For working class wives, adult children appeared to be a particularly valuable source of support, while for the wives of white collar men, husbands and non-relatives were the most important sources of support. Results of this study suggest that help from a 
wide range of sources is associated with better functional outcomes after life-thraatening illness.

Social Support and Adaptation to Cancer. The social support networks of cancer patients have been studied rather extensively (Wortman, 1984; Dunkel-Schetter, 1984). Yet many of these studies have been criticized for failing to consider the negative impacts on the support network of such a devastating and stigmatizing disease (Dunkel-Schetter, 1984; Dunkel-Schetter \& Wortman, 1982; Silver \& Wortman, 1980; Wortman, 1984). What has the available empirical work shown regarding the relationship between social support and coping with cancer? Most studies have found evidence that support is positively related to outcome, and some of the recent and more sophisticated work (Dunkel-Schetter, 1984) has found a relationship between the type and source of support and different outcomes in adaptation to this life-threatening illness. Funch and Mettlin (1982), for example, looked at three types of support provided to 151 female breast cancer patients between 3 and 12 months postsurgery: social support, financial support, and professional support. They found that social and professional support were significantly related to psychological adjustment, measured in terms of increased levels of positive affect and decreased levels of negative affect, while financial support was significantly related to physical recovery. Weaknesses of this study are that it was 
retrospective and correlational.

In another study, Funch and Marshall (1983) analyzed secondary data from a longitudinal study of 208 caucasian, female breast cancer patients. Measures of social support available to these researchers included marital status, number of relatives and friends, and organizational involvement. Subjects were divided into three age groups. When the entire sample was considered, stage of cancer was the best predictor of years of survival to death, the only outcome measure available. When only the youngest and oldest groups were considered, however, amount of reported stress prior to diagnosis and social involvement accounted for twice as much of the variance as did cancer stage at diagnosis. For these younger and older women, social involvement was positively related to survival, while reported stress was negatively related to survival. These researchers found no interaction between level of stress and social support in predicting survival, i.e., no buffering effect of social support. This is in agreement with Cohen and Wills' (1985) theoretical formulations regarding the stress-buffering role of social support. With the attribute measures and structural measures of social support used in this study, Cohen and Wills would predict no buffering effect would be found.

In a study which included measures of both personality variables and structural network characteristics (size and 
multiplexity) as well as outcome measures of anxiety and depressive symptoms, Hobfoll and halfisch (1984) did not find evidence that social support as they defined it was independently related to either anxiety or depression after controlling for the effects of personality variables (selfesteem, self-mastery). These investigators interviewed 55 women suspected of having breast cancer, at two points in time: just prior to biopsy (what they called the high stress period), and three months after a negative biopsy for cancer (the low stress period). In other words, they used subjects as their own controls to contrast psychological distress at high stress and low stress times. These researchers found that network size was not related to the measures of distress at either time period; however, multiplexity (the number of network persons whom subjects indicated they had many interests in common with) was related negatively to psychological distress but only during the high stress (pre-biopsy) period. The authors concluded that self-concept and social network variables are complexly related to outcome at periods of high stress, and perhaps equally critical. They also concluded that they had obtained main effects for the personality variables and a buffering effect for the social network variables. It is not surprising that these researchers did not find stronger effects for social support, given that it would have probably been much more appropriate to use functional 
measures of social support rather than structural network indicators as indirect measures of social support with this sample of patients fearing a diagnosis of cancer. Wortman (1984) has cautioned against using only structural measures of network contacts in investigating the importance of social support to cancer patients.

In a very well-done study which included multiple measures of social support and multiple measures of adjustment, Dunkel-schetter (1984) interviewed 79 persons with either breast or colorectal cancer. Seventy-five per cent of the subjects had a diagnosis of breast cancer, and $86 \%$ of the sample was female. With regard to prognosis, 47 were diagnosed as having a good prognosis and 32 as having a poor prognosis, based on staging of the cancer at the time of diagnosis. Because of the multiple measures utilized, patterns of relationships between type and source of support, prognosis, and adjustment could be delineated. Results indicated that although emotional support was seen as especially helpful to cancer patients, whether a particular type of support was seen as helpful was related to the source of the support. While emotional support and tangible aid were equally helpful across sources, information and advice for these cancer patients were almost exclusively reported as helpful when they came from health care personnel. Variability in levels of reported stress (as indicated by prognosis) mediated both the frequency of 
interpersonal problems and the association between social support and adjustment. That is, the higher the levels of support among those subjects with a good prognosis, the better their affect and the higher their self-esteem. For those with a poor prognosis, however, this relationship between support and psychological well-being did not hold. Likewise, support was negatively associated with physical condition, but only for those cancer patients with poor prognosis. Dunkel-schetter interpreted this association between higher levels of social support and poorer physical condition among those in the poor prognosis group as indicating that those with a poorer prognosis and poor physical status generated more assistance from the support network, probably appropriately. Because she employed multiple measures of both support and adjustment, DunkelSchetter obtained interpretable and useful results.

Bloom and Spiegel (1984) also found social support to be multidimensional. They interviewed 86 women with advanced breast cancer and found emotional support provided by the family to be strongly associated with one measure of psychological functioning - outlook on life, while opportunities for social exchange were related to both outlook and self-reported social functioning. Emotional support was seen to influence social functioning indirectly, through its influence on outlook on life.

Social Support and Other Disabling Illnesses. There 
are some studies available which describe the networks of persons dealing with a variety of disabling conditions. Not all of these studies are consistent, however, in showing that the structural and interactional aspects of the networks of disabled persons are different from those of the general population. One study which examined the social networks of a large sample of disabled persons (Morgan, Patrick, \& Charlton, 1984) using a network analytic approach, found that network size, rather than network type was related to the availability of psychosocial support. Network type in this study referred to primarily kin or nonkin networks, and whether kin networks were locally based or dispersed. The majority of disabled persons in this sample had intimate social networks of between 4 and 9 people, with the average being 7.2 persons. Only $11 \%$ had a network of less than four people. While the study provided some evidence for increasing isolation with increasing severity of disability, there was a wide range of support scores among all disability severity groups. Results also suggested that increasing physical disability had little effect on the availability of close, confiding relationships. Pertinent to questions that will be raised in this study, Morgan and coworkers found low levels of support among a number of married subjects, including a fairly large proportion who did not have a strong confiding relationship with their spouse. These authors questioned 
whether this was somehow related to disability and the stresses of illness.

Obtaining somewhat different results, schulz and Decker (1985), in their study of older spinal-cord injured men, found that their sample reported much smaller networks than did respondents in the study cited above. The mean number of network members reported by Schulz and Decker's respondents was 2.3 , the mode 1 . This discrepancy can perhaps be explained by the fact that $59 \%$ of subjects in the Morgan et al. study were classified as having mild disabilities. Another explanation for the discrepancy in the size of reported networks in these two studies may be the fact that the spinal-cord injured subjects were many years (mean of 20 years) post-injury, suggesting that support networks shrink with time for disabled persons. Also, total network size may have been underestimated in the Schulz and Decker study because of the method used to collect network data. They asked respondents to identify the most important persons in their network (R. Schulz, personal communication, November, 1985).

Another study which utilized a network analytic approach in describing the networks of families coping with a disability was that by Kazak and Wilcox (1984). They explored the networks of families with a handicapped child, examining several structural and interactional components of the social networks of 56 families in which a child had 
spina bifida. They also studied the social networks of a control group of parents without a handicapped child. Results indicated that the combined social networks of the parents of the handicapped children were significantly smaller, contained fewer friends, were more dense leven controlling for the number of kin in the network), had a greater overlap (boundary density) in the networks of the two parents, and contained more multidimensional relationships, that is, persons who provided more than one type of help. The mothers of the handicapped children had significantly lower levels of reciprocity with their family networks than did control group mothers. These results suggest that the parents of the handicapped children had fewer sources of support on which to call, and that they may be risking "overloading" family network members to continue to provide unreciprocated support. As friends have been found to be very important network members in a number of studies and throughout the Iifespan (Arling, 1976; Croog, Lipson, \& Levine, 1972; Schulz\& Rau, 1985; Wood \& Robertson, 1978), the finding that the parents of the handicapped children had significantly fewer friends may be an indication of stress within the networks of these parents which could impact on their well-being. The possible relationship between network characteristiss and parents' well-being was not explored by Kazak and Wilcox (1984) in this study, however. 
Data from a number of studies dealing with a variety of physical illnesses and disabilitios indicate that supportive relationships are important in adjustment to physical illness and chronic disability. For example despite the small sizes of their networks, the spinal-cord injured subjects of Schulz and Decker (1985) reported relatively high levels of well-being on three standard measures. Subjects who had high levels of social support lemotional, instrumental, and cognitive support were measured in this study), who were satisfied with their social contacts, and who indicated high levels of perceived of well-being, even after the effects of health status and income were accounted for.

In another cross-sectional study, Davidson, Bowden, and Feller (1981) examined the post-burn adjustment of 314 persons who had sustained moderate and severe burns and who had been treated at a major burn center from 1 to 20 years previously. Independent variables in this study included severity of injury, demographic information, and social support operationalized as family support, friend support, peer support, and overall support. Outcome measures included reported life satisfaction, self-esteem, and participation in social and recreational activities. These researchers found that social support related both directly and indirectly in the expected direction to all three 
outcome measures. They found evidence for a buffering effect of social support, in that effects were stronger in the severely and critically burned patients than in those with moderately severe burns. They also found differential patterns of relationship between outcome measures and the source of support, providing evidence again for the multidimensional nature of the support construct. For these burn patients, family support was more important in predicting outcomes than was friend support, and friend support was more strongly predictive of adjustment than was work and school peer support. Family support was especially strong compared to friend and peer support in predicting burn patients' reported self-esteem. On the other hand, family and friends were equally important in predicting participation in social and recreational activities, while peer support appeared not to be important at all.

Pancoast (1984) investigated the adjustment and vocational status of a group of 100 men with epilepsy, utilizing a network analytic approach and examining in some detail the role of social support in successful coping. She found that the social support networks of the men who reported high levels of life satisfaction were large, diverse, active, and generally helpful. Membership in a church was also a strong predictor of life satisfaction. Somewhat weaker relationships were found between the social support measures and employment success, although network 
density and general social participation were associated with successful employment. An indication of the negative impacts which one's social network can exert was also found in this study. Efforts of the professional helping network and of family and friends to provide specific help directed toward assisting the person to cope with epilepsy were found to be negatively associated with successful employment outcomes in individuals who perceived themselves as unable to control the epilepsy symptoms and to be limited by the condition.

A variety of other studies have been generally confirmatory of the notion that social support can mediate adjustment to chronic health conditions. Porrit (1979) in a study of 70 men who had suffered road injuries explored specific qualitative aspects of the support provided to subjects. These included support characterized by empathic understanding, constructive genuineness, and respect. Results indicated that the numbers of people available to provide support in a crisis had no effect on outcome, whereas the quality of network responses was related to crisis outcome for these subjects. As this study also had an intervention component, Porrit was able to determine that direct intervention in the form of practical and emotional support to the victim and suggestions to the families resulted in an increase in subjective reports of the quality of network responses for those patients in the two 
intervention groups. Porrit also argued that network responses appear to be additive in their effects, and that supportive and non-supportive reactions to illness crisis on the part of network members can cancel each other out. This finding is in disagreement with those of other researchers who have argued that having one close, confiding relationship is health-protective (Brown \& Harris, 1978; Lowenthal \& Haven, 1968).

McIvor, Riklan, and Reznikoff (1984) found significant negative correlations between level of depressive symptoms and the amount of perceived support from family and friends in a group of 120 multiple sclerosis victims. In terms of adjustment to the disability over time, these researchers found higher levels of depression in those who had had the disease longer. This was a cross-sectional study, however, and the fact that MS is a progressive disease may certainly have influenced depression scores.

Frankel and Turner (1983) found social support as measured using a story vignette format to be the most important predictor of adjustment in an adult hearingimpaired sample. Dimond (1979) interviewed 36 chronic hemodyalsis patients and found that each of a number of social support variables were positively associated with morale, especially family expressiveness. She also determined that family cohesion was negatively correlated with decreased levels of social functioning. None of these 
relationships were changed when medical status was controlled for.

Heitzmann and Raplan (1984) found that the number of support persons in the network did not influence physiological measures of control of Type II diabetes mellitus (an indirect measure of compliance) in a sample of 37 persons, 19 males and 18 femaies. For female patients, however, high scores on a measure of satisfaction with available support were significantly associated with control of diabetes. In a study which failed to find either a direct or a mediating effect for social support, iambert (1984) studied a group of women with rheumatoid arthritis. In this sample, only level of reported pain was a predictor of psychological well-being. This is consistent, however, with other studies which have found that in persons with severe pain or with greatly deteriorated health, social support factors are not powerful enough to influence reported well-being (Dunkel-schetter, 1984; Lowenthal \& Haven, 1968).

Social support and stroke. There are few studies available which have specifically focused on the social support systems of persons who have experienced a stroke, and only one which is more than descriptive and correlational (McLeroy, DeVellis, DeVellis, Raplan, \& Toole, 1984). Some inferences regarding the social support systems of stroke patients and caregivers can be made from studies 
on the impacts of stroke which were previously reviewed. There are almost no studies which have systematically examined the social support networks of caregivers of stroke patients.

One study did examine the relative influences of social support, social network characteristics, physical, and psychological factors on the morale and life satisfaction of 50 elderly spouses of chronic stroke patients (Unks, 1983). In this research multiple measures of socjal network structures, supportive network functions, and physical and psychological indicators as well as demographic characteristics were included. Results indicated that the wife's perception of her husband's impairment level was the best predictor of caregiver wellbeing. While informal social support variables were related to morale, they did not appreciably alter the relationship between the severity of the stroke victim's physical impairment and the spouse's morale. Unks speculated that the increase in caregiver burden, changes in the marital relationship, and role changes implied by increased levels of physical impairment in the person being cared for were probably responsible for the strong association between victim impairment level and spouse's morale.

McLeroy and his co-workers (1984) examined the role of social network characteristics and types of social support on the physical recovery of 393 stroke patients at 12 months 
after discharge. The outcome, physical recovery, was measured by an Activity of Daily Living (ADL) scale. Social network and social support measures included: frequency of social activities by type of activity; frequency of contact with family and relatives living outside the home; emotional support from physicians, nurses, other stroke patients, church members, family, friends, and neighbors; and stroke-related instrumental and informational assistance, by type of provider. Social network characteristics (frequency and type of social activities and family contacts) had no direct effects on ADL status at 6 months. Instrumental assistance continuing to be provided at six months post-stroke, however, had a negative relationship with ADL status, even after controlling for the effects of age, cognitive ability, and illness experience since hospital discharge. Furthermore, for those subjects who had received instrumental assistance at some time during the post-discharge period but who were not receiving such assistance at 12 months, there was a positive relationship between instrumental assistance and outcome ADL status. These results were stable across times of measurement and across outcomes (neurological and ADL status).

Because of statistical controls possible with a longitudinal design, including controlling for ADL status at the time of hospital discharge, these researchers were able to demonstrate that stroke-related instrumental assistance 
when provided throughout the follow-up period had a detrimental effect on ADL functioning at one year. This study, because it was well designed, was able to demonstrate that (1) there is a relationship between type of support and type of outcome (e.g., physical status vs. subjective wellbeing); and (2) that the possible negative impacts of supportive actions must be considered in assessing outcomes. Some data on the characteristics of stroke patients' social networks are available from a demonstration project which involved stroke patients and families lovers and Healy, 1973). The picture that emerged from this study was one of quite isolated individuals and families. Only $11 \%$ of the help received by male stroke victims and $16 \%$ of the assistance received by female stroke patients was provided by someone outside of the family. Most of the support functions reported were tangible/instrumental: transportation, shopping, chores, financial help, patient care, child care and providing living accomodations. Very few subjects acknowledged information or advice as a type of support. Fifty-nine per cent of the subjects were described as isolated from neighbors, with no other contacts than family or work groups. Only $10 \%$ of the sample were considered not at all isolated, that is, they had independent contact with more than one family in the neighborhood. Male stroke patients saw an average of 2.9 persons a week, while female stroke victims saw 2.2 persons 
a week. Of these limited contacts, $65 \%$ were a spouse or child and about $80 \%$ were family members. There was some evidence from the study that stroke families and patients were able to maintain some degree of reciprocity in their neighboring relationships, in that the giving and receiving of services were in about equal proportions.

Social network characteristics of the spouses of a sample of chronic stroke patients were studied by Unks (1983). His subjects, all female, reported having between 8 and 30 people in their networks, with a mean of 18.5 and median of 16.5. The average network size approximates network estimates in a representative community sample (Fischer, 1982) and in a large survey sample of elderly women (Goldberg, Van Natta, \& Comstock, 1985). These wives of stroke patients reported that large numbers and proportions of their network members provided emotional support and socializing opportunities, while tangible assistance and information were provided by almost half of named network members. of all the types of supportive functions provided, the number of people providing emotional support was most strongly associated with the caretakers' overall morale. Consistent with other available information on the social networks of older persons, friends were very important in the support systems of the women in Unks' sample, comprising $40 \%$ of their close, or inner network members. Contact with network members was extensive. 
subjects saw their network members and exchanged some conversation an average of 49 times per month, and talked on the phone with them an average of 52 times a month. Despite large networks and frequent contacts, almost half of the caregivers reported that they had no one whom they viewed as a confidant. The presence of a confidant was not associated with morale for this sample of women, however - a somewhat surprising finding.

Several studies have provided evidence that the reactions and behaviors of network members can positively or negatively influence stroke outcomes. Hyman (1971) reported an association between stroke patients' feelings of stigma and relatives perceptions of them as stigmatized. Labi, Phillips, \& Gresham (1980) reported a greater degree of social disability in stroke patients who lived with their spouses. Stroke survivors whose significant other was a spouse experienced the greatest decrease in social activity. Andrews and stewart (1979) found that in more than half their sample, caregivers claimed that stroke patients did not do two or more activities at home which they were observed to be capable of doing in a day hospital program in which they participated. Belcher, Clowers, Cabanayan, and Fordyce (1982) determined that single stroke survivors had higher levels of participation in performing personal care, home management, and social interaction activities than did married stroke subjects. In this study, however, it could 
not be determined whether the two groups were comparable in terms of ADL independence at discharge. New et al. (1968) examined the degree of agreement in patient $A D L$ ratings as completed by a total of 98 significant others and by 40 stroke patients and 8 heart patients. They found disagreement between patients and significant others as to the patient's capabilities. These studies taken together lead one to conclude that a possible source of conflict which may have negative impacts on the provision of social support and the appropriateness of social support provided is the degree of congruence between the functional capacities of the stroke patient as he or she sees them and as the significant caring persons see them.

A similar question was explored by Evans and Northwood (1979), who examined the congruence between expressed level of social interaction and desired levels of social support in a group of 48 recently discharged stroke patients. Results indicated a significant relationship between the congruent expression of social support needs and adjustment to stroke disability and between the availability of social support after stroke and adjustment. In another study (Robertson \& Suinn, 1968), congruence operationalized as predictive empathy of stroke patients for their spouses' attitudes and of spouses for stroke patients' attitudes was significantly related to the patients progress in rehabilitation, although similarity of spouses' and 
patients' attitudes was not.

Summary: Social Support and Adaptation to Physical Illness

In summarizing the work on the relationship between social networks, social support, and adjustment to physical illness and disability, several generalizations, and several criticisms $\cdots n$ be made. For the most part, it appears that measures of the functional aspects and quality of support provided or perceived are more important than structural measures of network size or frequency of contact. Other network characteristics such as source and type of support provided and network density and multiplexity may be important determinants of certain types of outcomes in chronically ill and disabled populations. The studies as a whole confirm the notion that social support is multidimensional, and that many aspects of support including the nonsupportive functions of networks must be considered in designing social support studies. Finally, although the focus of this review was on social support, most studies found other variables to be related to well-being and social adjustment outcomes. Variables which need to be considered include age, sex, SES, length of illness or disability, severity of disability, individual coping mechanisms and personality variablies, and the specific characteristics of the illness or disability being studied.

Criticisms of the studies reviewed include methodological and design weaknesses as well as problems 
with the conceptualization of social support. Almost all of the studies were cross-sectional and correlational, precluding the possibility of determining the causal relationships among social support, aspects of the illness, and well-being outcomes. This is a common problem in the social support literature, and several reviews have emphasized the need for longitudinal studies related to coping and recovery from illness (DiMatteo \& Hays,1981; Wortman \& Conway, 1985). Many of the studies had small sample sizes which necessitated the use of only descriptive and non-parametric statistics in reporting results. Several depended upon retrospective data which required subjects to recall information several months to several years after the occurrence of events or situations.

While a few of the studies employed detailed and multidimensional definitions of social networks, social support, and adjustment (Dunkel-schetter, 1984; Funch \& Mettlin, 1982; Pancoast, 1984; Schulz \& Decker, 1985), most of the studies were much weaker in this regard. Some employed only one outcome measure, such as survival (Funch \& Marshall, 1983) or ADL status (McLeroy et al, 1984), while others only measured one aspect of support (Funch \& Marshal1, 1983; Hobfoll \& Walfisch, 1984; Dimond, 1979).

There is a need for a longitudinal study of adaptation to chronic illness and disability which includes measures of: network characteristics; supportive functions provided 
by the network, including instrumental, emotional, informational, and companionship support; individual coping mechanisms; and both physical and psychological outcomes. Such a study must consider the influence of the chronic, disabling condition on the network as well as the role social support may play in enhancing or interfering with adaptation and well-being. Such a study should not only include information about the disabled group, but should also focus on the primary caregivers of such persons. Finally, it would seem to be most appropriate to investigate these aspects of coping with chronic illness in a homogeneous group of persons, that is, a group that is dealing with the same disabling condition, has experienced the condition for approximately the same length of time, and from whom reasonably prospective data can be gathered. Chapter II will present the theoretical framework for such a study, and the remaining chapters will detail the methodology and results of this investigation of recent stroke victims and their caregivers. 
CONCEPTUAL FRAMEWORR OF THE STUDY

FACTORS PREDICTIVE OF STRORE OUTCOME

We have seen from the preceding literature review that certain factors will be important to include in any predictive model of the relationship between the occurrence of a disabling stroke and subsequent measures of well-being and adaptive functioning. Among these factors are the characteristics of the stroke disability itself, perceptions of the stroke, several aspects of social support, and certain characteristics of the individual. Specific aspects of each of these factors may be different for stroke patients and caregivers, however. In addition, some factors will relate neither to the patient nor to the caregiver alone, but rather to aspects of the couple's relationship and interaction. Table II and Table III outline which aspects of the stroke disability, perceptions of the stroke, social network resources, and characteristics of the individual will be important to consider for the stroke patient (Table II) and the spouse caregiver (Table III). Table IV summarizes some possible predictive factors related to the stroke patient-caregiver relationship.

\section{Factors Predictive of Stroke Patient Outcomes}

stroke-related Factors. For the person who has 
suffered a stroke, a major factor in predicting adjustment will be the severity of the stroke itself. that is the extent of residual impairment in physical and cognitive functioning. In addition, the literature suggests that the site of the stroke lesion (i.e., right or left hemisphere) may be predictive of such outcomes as level of depressive symptomatology and ability to interact in socially appropriate ways. Duration of the stroke may be important as well, in that differences in well-being and coping may be seen between the initial phase of stroke recovery and at six months post-discharge.

Perceptions of the stroke. There is evidence to suggest that how the individual perceives and interprets a stressful life event, including illness and disability, may be more important than objective indices of impairment, incapacity, or deprivation (Hyman, 1971; 1972). For the stroke victim, such perceptions may involve feelings of guilt or self-blame that the stroke occurred, concerns about another stroke, expectations about future recovery, and perceptions of the overall severity of the stroke which may or may not agree with measures of ADL functioning or with how significant others perceive the severity of the stroke.

Aspects of Social Support. Previous level of social integration has been suggested as important in determining the types and levels of support an individual faced with a chronic, disabling illness will receive (Croog, Lipson, \& 
TABLE II

EACTORS PREDICTIYE OF STRORE DATIENT HEIL-BETNG

\section{Stroke Related Factors}

Severity of the stroke

Site of Lesion (Right vs. Left Hemisphere)

Time since stroke onset (Duration)

\section{Perceptions of the stroke}

Attributions of Guilt or Blame

Expectations of Recovery

Concern about Another Stroke

Perception of Overall stroke Severity

Aspects of the Social Network and Social Support

Pre-stroke

Level of Social Integration

Structural Characteristics of the Network

Types and Levels of Social Support

Perceived Adequacy of Social Support Quantity and Quality

Negative Aspects of Network Relationships

Post-stroke Changes in Network and Social Support

\section{Characteristics of the Individual}

Degree of Religiosity

Level of Optimism

Stroke-Related Changes in Role status

overall Health Status 
Levine, 1972). Therefore, it will be important to consider pre-morbid levels of social activity and supportive social contacts. It will also be necessary to consider the types, sources, and levels of social support provided before and during various periods following the stroke, specifically during the initial adjustment phase and six months later.

Changes in the amount and frequency of social network interaction, or in the quality of that interaction may well be predictive of adjustment and adaptation. Network characteristics such as density and the amount of reciprocity in network exchanges may be important indicators of network stability or change. Perceived satisiaction with the amount and quality of social contact needs to be examined. Another important aspect of social support is predicted to be the degree of congruence between the stroke victim and the support network as to perception of the severity of the stroke-related disabilities and the amount of assistance and help required. Assistance which is perceived as unnecessary, inadequate, or inappropriate by the stroke victim may precipitate network strains, and result in negative outcomes.

Characteristics of the Individual. The literature does not provide much evidence that demographic characteristics such as age, sex, or socioeconomic status will predict adjustment in stroke patients when other factors such as severity of stroke-related disability are 
taken into account. Other individual characteristics such as degree of religiosity and level of optimism as a coping trait may be important in helping the individual adjust to negative stroke-related changes, and to come to terms with life-style and role changes. Another important characteristic of the individual is the variety of roles he or she has fulfilled prior to the stroke. Changes in role status, such as having to give up work-related and leisure roles because of the stroke, or no longer being able to fulfill previously established marital roles may be related to negative mental and physical health outcomes (Minkler \& Biller, 1979).

\section{Factors Predictive of Caregiver Outcomes}

stroke-related factors. The literature provides contradictory evidence as to whether the severity of strokerelated problems will impact on caregiver well-being (Rinsella \& Duffy, 1980; Unks, 1983). There is some suggestion that negative personality and behavioral changes in the stroke victim may be more stressful for the caregiver than changes in physical abilities. Site of stroke lesion and its differential impact on caregiver health status is another stroke-related factor which needs to be considered. Some studies have found that left hemisphere strokes, with their negative impact on communication, result in more caregiver strain (Artes \& Hoops, 1976; Rinsella \& Duffy, 1978, 1980). Other researchers have found, however, that 
TABLE III

FACTORS PREDICTIYE OF CAREGIVER WELI-BEING

\section{Stroke-Related Factors}

Severity of Stroke-Related Problems

Site of Stroke Lesion

Time since stroke onset (Duration)

\section{Perceptions of the stroke}

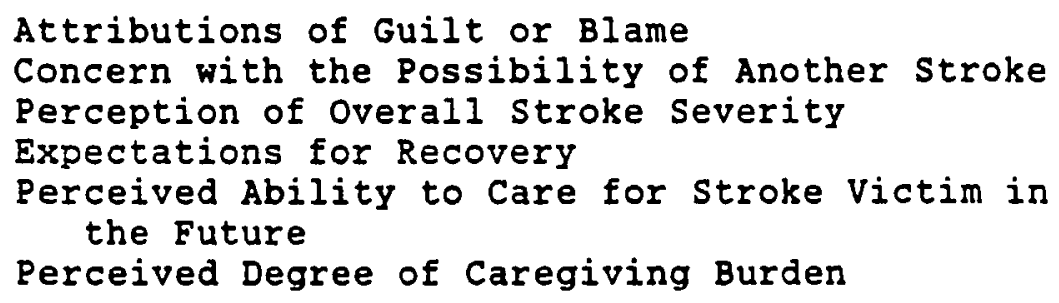

Aspects of the Social Network and Social support

Pre-stroke

Level of Social Integration

Structural Characteristics of the Network

Types and Levels of Social Support

Perceived Adequacy of Social support

Negative Aspects of Network Relationships

Post-Stroke Changes in the Network and Social Support

\section{Characteristics of the Individual}

Religiosity

Level of Optimism

Pre-Stroke Health Status

Post-stroke Changes in Health Status

Stroke-Related Role Changes 
right hemisphere strokes are more disruptive of the caregiver-stroke patient relationship (Coughlan \& Humphrey, 1982). The duration of the stroke, or the time since onset may affect caregiver well-being in opposite ways. On the one hand, it is suggested that the longer the time since stroke onset, the worse will be the mental health status of the primary caregiver (Rinsella \& Duffy, 1978). On the other hand, it may be that time will provide a period of adjustment and adaptation for the spouse of the stroke survivor.

Perceptions of the stroke. For the primary caregiver as well as for the patient, perceptions of guilt or blame for the stroke's occurrence, concern about the possibility of another stroke, and perception of the stroke's overall severity may be related to well-being outcomes. In addition, concern about being able to continue to care for the individual who has suffered the stroke may increase levels of distress. Another important aspect of the caregiver's perceptions related to the stroke is the degree of caregiving burden he or she feels (Zarit, Reever, \& BachPeterson, 1980). Research has indicated that the degree of burden perceived by the caregiver is not necessarily related to the severity of the impairment of the disabled or dependent individual (Zarit, Todd, \& Zarit, 1985). The partner's perceptions of personality or behavioral changes in the stroke victim may be related to the degree of felt 
burden and to well-being outcomes.

Aspects of Social Support. Various components of the primary caregiver's social network need to be considered independently of the social support system of the stroke patient. Network size, density, frequency of supportive exchanges and contacts, degree of reciprocity characterizing network exchanges, and the proportions of kin, neighbors, and friends in the caregiver's network before the stroke may be important indicators of the amount and quality of support available in the immediate post-stroke period. How the caregiver perceives the availability, adequacy, and quality of support may be more related to well-being than the actual amount of network support she calls upon. Several studies (Fiore, Becker, \& Coppel, 1983; Rook, 1984) have suggested that the amount of socializing or "sociable contacts" is more strongly related to caregiver well-being than other structural or functional network measures. Changes in these aspects of social support at six montins post-stroke, especially those which can be viewed as negative changes, may be related to declines in well-being and/or increases in depressive symptoms.

Crisis events, such as a stroke, or widowhood, can prompt responses from the network which are not always supportive, and which may be viewed as stressful by the person who experiences them. In fact, two recent studies (Fiore, Becker, \& Coppel, 1983; Rook, 1984) found stronger 
relationships between negatively perceived network interventions and unmet support expectations and well-being outcomes than between positive network support measures and outcomes. It will be important then, in any model describing the relationship between social support and health outcomes to consider the unsupportive, stressful aspects of network relationships for the partner.

Characteristics of the Individual. As was predicted for the stroke patient, certain individual characteristics of the caregiver such as religiosity and degree of optimism may be important in determining how the chronic stresses of caring for a disabled partner are dealt with. These individual characteristics may, in turn, be important determinants of well-being. The caregiver's own health may be both predictive of coping with the additional stresses of the caregiving role and at the same time be negatively affected by the stresses of caregiving. The number and magnitude of stroke-related role changes experienced by the caregiver also may be related to levels of depressive symptoms and to psychological well-being. As was reviewed in Chapter $I$, one of the most frequently reported concerns of the caregivers of stroke patients is difficulty in dealing with the many role changes associated with a disabling stroke.

Factors Related to the Stroke Victim-Caregiver Relationship Several factors which may be important to stroke 
outcomes require consideration of the networks of both the stroke patient and the partner, as well as the quality of their relationship. Table IV summarizes these factors. Because the marriage relationship is generally considered a powerful determinant of well-being and an important source of social support (Berkman \& Syme, 1979; Campbell, 1981; House, Robbins, \& Metzner, 1982; Veroff, Douvan, \& Kulka, 1981), the perceived quality of the couple's relationship and any negative changes in the relationship resulting from the stroke may have negative impacts on outcomes for both the caregiver and the patient. The degree of congruence between the stroke patient's and the partner's ratings of the quality of their relationship will be important to consider.

The concept of boundary density, or network overlap may have relevance for the social support available in coping with a stroke. As defined in Table I, boundary density refers to the number or proportion of network members who are found in both of the two focal individuals' networks. The literature on dense versus diffuse networks (Bott, 1957; Hirsh, 1980; Wilcox, 1981) and on reactions to victims (Dunkel-Schetter \& Wortman, 1981) would suggest that caregivers who have maintained relatively separate social networks from their spouses might receive more appropriate types and amour.ts of support after the occurrence of a stroke than caregivers whose networks are 
TABLE IV

FACTORS RELATED TO THE STRORE PATIENT-CAREGIVER RELATIONSHIP

Aspects of the Couple Relationship

Individual perceptions of overall relationship quality Congruence of perceptions of overall relationship quality

Level of communication

Frequency of disagreements

Amount of shared or joint activity

Network Overlap

Congruence of Perceptions Regarding the stroke

Impact of Individual Well-Being on the Partner

Level of Optimism

Depressive Symptom Level

Psychological Well-Being 
more overlapping with those of their spouses. If network memiers were close to both the stroke patient and the caregiver, they may experience levels of discomfort, distress, and helplessness related to the stroke which diminishes their ability to provide support.

In predicting stroke outcomes, it will also be important to look at the congruence between the stroke patient's and the spouse's perceptions of the severity of stroke-related disabilities, and the amount of assistance and supervision required compared with the level of functional independence the stroke victim has achieved (Andrews, \& Stewart, 1979; Mcheroy et al, 1984). Incongruent perceptions of the patient's capabilities may result in the stroke victim feeling under or over supported, and the caregiver feeling overburdened and lacking in support.

\section{A GENERAL MODEL FOR THE STUDY}

Based on the literature review and on consideration of the multiple factors which may be important in predicting adaptation and well-being following a stroke, a general model of the adjustment/adaptation process is proposed in Figure 1. This model is based on one offered by Gore (1981), which views the relationship between the stressor (in this case, the occurrence of a disabling stroke) and the support system as interactive and dynamic. Arrows going 


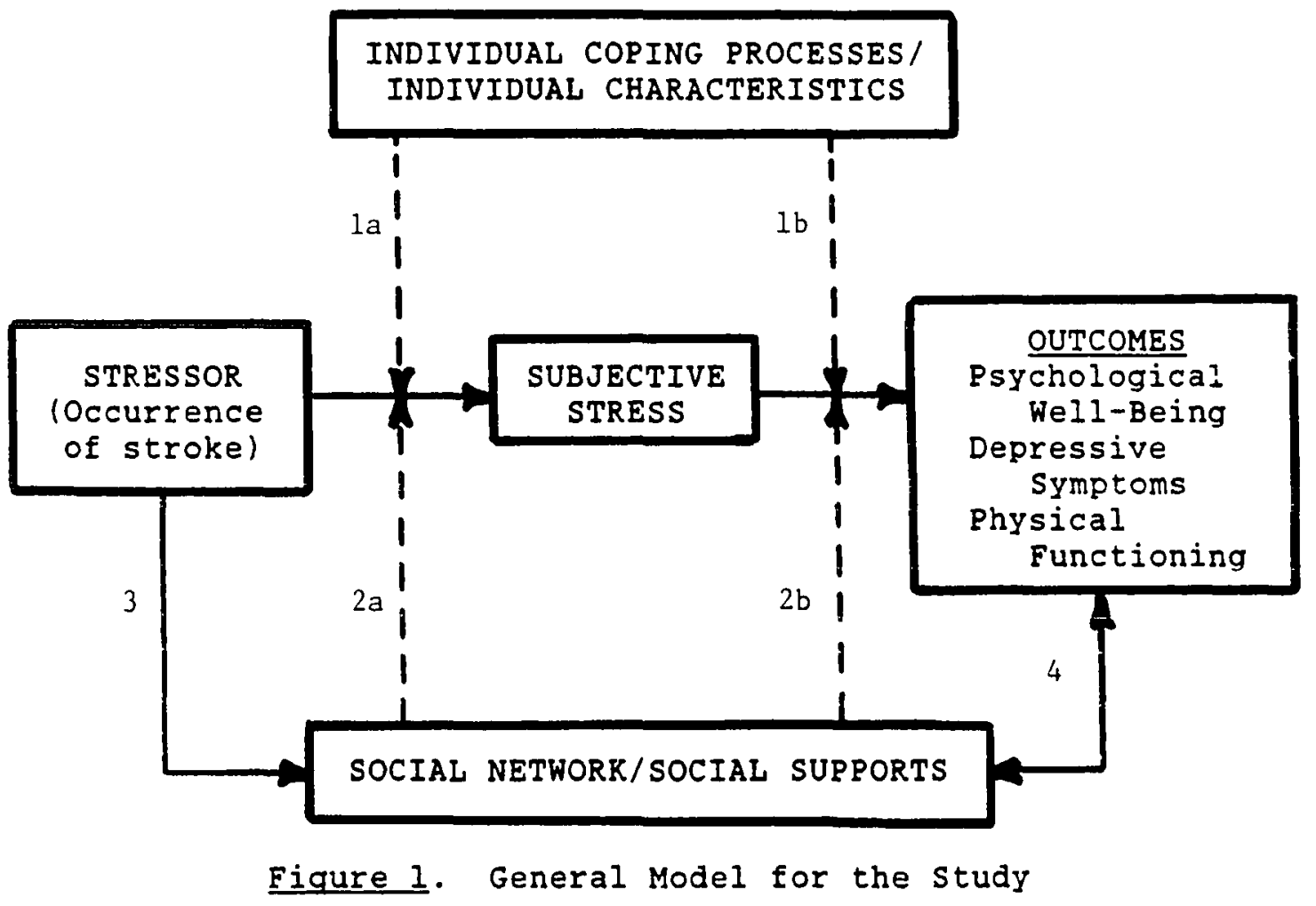

Solid lines represent direct, causal relationships.

Dashed lines represent "conditioning" variables effects. 
from social support and from individual coping processes (individual characteristics) act to mediate or condition the impact of the stressful event (the stroke), either before or after stressful effects of the stroke are perceived. Arrows 1a, $1 b, 2 a$, and $2 b$ are dashed lines to indicate these "conditioning" variables' effects. Arrow 3 depicts the direct effects of the stressor on the support systems of the stroke victim and caregiver, while arrow 4 represents the direct effects of the social support system on measured outcomes. In some models, arrow 3 would be seen as a twoway path, indicating not only the effects of the stressful event on the social network, but also the possible role of social network characteristics and social support processes in bringing about the stressful event. In this study, however, we are concerned with social support system changes related to and following the stressor. Likewise, arrow 4 should be seen as a two-way path indicating the interactive relationship between the social support system's response to the stressor and victim/caregiver outcomes as well as the relationship between the stroke victim's and caregiver's response to the stroke and subsequent social support system activity.

\author{
QUESTIONS ADDRESSED BY THE STUDY
}

Several important unanswered questions relate to the longitudinal nature of a chronic, disabling illness and its 
effects on the social support systems of both the victim and the significant other person involved with that individual. This study proposes to address the following questions:

1. In what ways does the support system change over time as a result of a chronic illness?

2. What is the impact on the patient and on the significant caring person of a changing support system? The strong possiblity exists that there are negative changes over time in the social network as a result of a chronic disability. Assessment of the quantity and quality of social support available only in the initial phases of the illness may not be predictive of longer term outcomes or later need for intervention. It would seem to be important to assess the social support system over time as the health status of the patient moves from acute crisis to chronic condition, in order to determine which aspects of social network support may be most vulnerable to negative changes related to the stroke.

3. What are the correlates of health and well-being outcomes at different points in time in a sample of recent stroke patients and their spouses? What are the best predictors of outcomes at Time 1 and Time 2?

4. What are the best predictors of health and wellbeing outcomes over time for both the patient and the partner? This study will seek to develop some predictor variables which will help identify those persons most likely 
to be in need of preventive interventions or other types of assistance.

5. What is the relationship between site of lesion (specifically right or left hemisphere brain damage) and well-being outcomes cor both the stroke survivor and the caregiver? How does site of lesion affect various structural components of the social network and levels and quality of social support?

$$
\text { HYPOTHESES TO BE TESTED }
$$

A number of hypotheses are generated by the above questions and by the model in Figure 1 . These hypotheses are discussed in terms of stroke-related factors, perceptions of the stroke, aspects of social support, characteristics and attributes of the individual, and characteristics of the partner relationship.

\section{Hypotheses: Stroke-Related Factors}

Hypothesis 1a. It is predicted that the greater the severity of the stroke-related disability at six months post-onset, the higher will be the scores for both patient and caregiver on outcome measures of negative well-being, and the lower will be their scores on positive measures of well-being.

Hypothesis 1b. The site of the stroke lesion lright or left hemisphere) will be related to caregiver well-being at follow-up. Left hemisphere strokes will be associated 
with lower caregiver well-being, because of the central importance of communication abilities and opportunities in sustaining a relationship.

Hypotheses: Perceptions of the Stroke

Hypothesis 2a. It is predicted that greater concern regarding the occurrence of another stroke and about the ability of the partner to provide continued care will be associated with poorer outcomes for the stroke patient.

Hypothesis 2b. A persistent sense of self-blame and guilt for the stroke having occurred will be associated with less positive outcomes for the patient.

Hypothesis 2c. Persistent concern regarding another stroke and about ability to continue providing care for the patient will be negatively associated with well-being outcomes for the partner.

Hypothesis 2d. Continued feelings of guilt or blame for the stroke having occurred will be associated with lower caregiver well-being over time.

Hypotheses: Aspects of Social Networks and Social Support

Hypothesis 3a. There will be some negative changes in the social networks of both stroke patient and partner over time: decreases in network size, decreased frequency of network contacts, and increased network density. Relationship composition of the network will change to include fewer friends and a greater number of kin. 
Perceived reciprocity in network exchanges will decrease.

Hypothesis 3b. The higher the reported levels of prestroke social activity, integration, and support, the more positive will be well-being outcomes for both patient and caregiver during the early post-stroke period.

Hypothesis 3c. Negative changes over time in the structural and interactional aspects of social networks (size, density, frequency of interaction) will be less predictive of outcomes for both patient and caregiver than will negative changes in the qualitative and content aspects of social support (degree of satisfaction with support, maintenance of reciprocity in network exchanges, maintenance of sources of different types of support).

Hypothesis 3d. It is hypothesized in exchange theory terms, that the lower the level of functional independence of the stroke patient in the early post-stroke period, the greater will be the amount of social support reported at that time, while at follow-up it is hypothesized that these relationships may be reversed, with a higher level of funstional independence being associated with greater reported social support. The rationale for this hypothesis is based upon the reciprocal nature of exchanges postulated by exchange theorists. (Gouldner, 1960: Shumaker \& Brownell, 1984). Chronic disability violates the exchange norm that the reciprocity of exchanges includes a time-limited provision of unreciprocated support or 
assistance. Over time, this results in the erosion of network linkages of certain types (i.e., those of friendship or neighboring, as opposed to kin relationships).

Hypothesis 3e. It is also hypothesized that the presence of a significant communication disability at follow-up will be associated with more negative social network changes llack of reciprocity, small network size, high network density, and low levels of social contact frequency and reported network support), and with more negative outcomes for the caregiver in terms of reported health status and psychological well-being. The rationale for this hypothesis can also be stated in terms of exchange theory. That is, chronic disability leading to nonreciprocal exchange relationships will be more congruent with societal norms regarding exchanges if close kin and intimates are the providers of the nonreciprocal care. This situation increases the burden experienced by close family members of the chrorically disabled person, and will be related to more negative caregiver outcomes.

Hypotheses: Individual Characteristics

Hypothesis 4a. Level of optimism and level of religiosity will be positively related to well-being outcomes for both patient and caregiver over time.

Hypothesis 4b. Negative changes in role status (employment, hobbies and leisure activities, marital partner role) will be negatively associated with well-being 
outcomes, but more strongly for the stroke patient than for the caregiver.

Hypothesis 4c. Demographic characteristics will not be associated with well-being outcomes when other factors, such as the severity of the stroke are controlled for.

Hypotheses: Stroke Victim - Partner Relationship

Hypothesis 5a. Congruence in the perceptions of patient and partner concerning the quality of their relationship will be positively associated with well-being.

Hypothesis 5b. The degree of overlap in the social networks of the patient and caregiver will be negatively associated with well-being outcomes over time.

Hypothesis 5c. Well-being outcomes of patient and caregiver will be positively associated at different points in time, and over time.

The following chapter will outline the design of the study undertaken to test these hypotheses, and describe the methodology employed. 
CHAPTER III

METHODOLOGY

This study, which focused on stroke patients and their spouses or partners, was part of a larger, three year investigation examining social support and well-being changes over time in those suffering a first, disabling stroke and their caregivers. The study was supported by grants from the National Institute on Aging and the AARP Andrus Foundation.

The overall investigation employed survey research methodology and a panel design. Data were gathered for this study at two points in time, with interviews scheduled six months apart. The primary data gathering tool was a questionnaire administered during structured, face-to-face interviews. Additional information was obtained from previously developed scales adopted for this study and from stroke patient medical records. Details regarding characteristics of the sample, data gathering procedures, questionnaire content, and operational definitions of variables are contained in the following sections of the chapter.

\section{THE SAMPLE}

\section{Subjects}

Subjects were 50 community-dwelling lat the time of 
the stroke) dyads consisting of individuals who had recently sufiered an initial, completed stroke, and their spouse or partner. Criteria for inclusion in the study were the following: (1) clinical evidence that this was a first completed stroke: (2) no evidence of co-existing terminal illness or other rapidly progressive medical disease; 3) the stroke having occurred within the eight weeks prior to the initial interview; (4) geographical availability to participate in face-to-face interviews (living within a 50 mile radius of Portland, Oregon): (5) availability of a spouse or partner willing to participate in the study, and willing to provide information regarding the stroke victim member of the dyad in those cases where the stroke patient was unable to respond; and (6) willingness of those stroke patients who were able to be interviewed to give consent, and to agree to participate in the study. In addition, only those dyads for whom both Time 1 and Time 2 interview information were available were included in this study.

\section{Demographics}

stroke-patient subjects had a mean age of 63.74 years and a median age of 63.83 years, with a range of 37 to 90 years. The caregivers (also referred to as "primary informants") in the sample had a mean age of 62.5 years and a median age of 62.5 years, with a range of 36 to 84 years. Forty one (828) of the stroke patient subjects were male, while nine (18\%) of the caregivers were male. The sample 
was predominantly caucasian, with 948 of the patients and 92\% of their partners being white. With regard to the stroke patient - caregiver relationship, all subjects were married or living as married at the time of initial interview. Other demographic characteristics of the sample will be discussed in detail in Chapter IV.

\section{Representativeness of the Sample: Demographics}

of the total of 210 dyads referred to the portland Stroke study, 166 (798) met all eligibility criteria. Table $v$ summarizes reasons for exclusion from the study. From this pool of potential eligible subjects, 124 (75\%) could be contacted and agreed to participate.

of the 166 eligible dyads, 37 (22\%) declined to participate at the time of initial contact. Table VI lists the reasons given by those who declined. There was a tendency for those who were feeling extreme stress because of the occurrence of the stroke, and for those coping with medical complications of the stroke and other health problems to decline to be interviewed within the time constraints of the study, although several indicated a willingness to be contacted "later". Balancing these possible biasing factors was the tendency for some stroke survivors who were doing extremely well to see little value in participating despite careful explanation of the purposes of the study.

Marital couples comprised 78 of the 124 study dyads, 
TABLE V

NUMBER AND PERCENT OF EXCLUDED STRORE CASES BY REASON FOR EXCLUSION, PORTLAND STROKE STUDY

REASON EOR EXCLUSION

History of previous strokes

No clear diagnosis of stroke

No significant other available

Stroke victim died before $T 1$ interview

Stroke occurred outside study time limits

Outside geographical boundaries of study

Total Cases Excluded
NUMBER

PERCENT

5

11

2

5

6

14

6

14

5

11

20

45

44

100 
TABLE VI

NUMBER AND PERCENT OF DYADS DECLINING TO PARTICIPATE BY REASON EXPRESSED, PORTLAND STRORE STUDY

$\begin{array}{lcc}\text { REASON } & \text { NUMBER } & \text { PERCENT } \\ \text { No time, too busy } & 10 & 27 \\ \text { Too soon, too much stress } & 8 & 22 \\ \text { Doing too well } & 1 & 3 \\ \text { Stroke victim health problems } & 5 & 13 \\ \text { Caregiver health problems } & 1 & 3 \\ \text { No reason stated } & 12 & 32 \\ & & 100\end{array}$


or 63\%. At the time of data analysis for this investigation, 61 of 78 couples could have completed the second (Time 2) interview. The 50 dyads which make up the sample for this study were obtained from this pool of 61 potential couples. Reasons for not completing both the Time 1 and Time 2 interviews (11 of $61,18 \%$ ) were as follows: Seven refused further participation at the time of the T2 interview; two stroke victims had died during the time between interviews; one couple had severed their relationship and only the primary informant (PI) was available for the T2 interview; and one PI was ill and could not be interviewed at T1. The success rate of $82 \%$ for obtaining both $\mathrm{T} 1$ and $\mathrm{T} 2$ interviews from the marital dyads compared favorably with the overall completion rate of $86 \%$ for the Portland subjects as a whole.

In order to determine the representativeness of the study sample, examination was undertaken of certain demographic characteristics of the marital dyads compared to the dyads which declined to participate in the study. Information regarding mean age, gender, and marital status characteristics of the two groups is summarized in Table VII.

Age information was available for 29 of 37 of the stroke patients in those dyads which declined participation. The mean age of the stroke victims in the refusal group was 69.21, compared to a mean age of 63.75 for the stroke 
TABLE VII

COMPARATIVE DEMOGRAPUIC DATA EOR MARITAL DYAD SAMPIE AND GROUP WHICH DECLINED TO PARTICIPATE

$\begin{array}{ll}\text { MARITAL } & \text { REFUSERS } \\ \text { DYADS } & \\ (\mathrm{N}=50) & (\mathrm{N}=37)\end{array}$

$\overline{\mathrm{X}}$ Age

PATIENTS

CAREGIVERS
63.75

62.5
69.21

*

\section{Gender}

PATIENTS

Male

Female

CAREGIVERS

Male

9 (18)

$10(27)$

Female

41 (82)

27 (73)

\section{Marital status}

Married/Living as Married

$50(100)$

$25(68)$

Not Married

$0(0)$

$12(32)$

* Ages not available for caregivers in refusal group

* Numbers in parentheses are percents. 
patients included in this study. Data on marital status were available for 165 of 166 of those referred and eligible for study participation. Regarding the marital status of those who declined, 25 of 37 dyads (68\%) were married or living with a partner compared to $100 \%$ for this study sample and $63 \%$ for the total portland sample. In summary, comparison of demographic characteristics of those couples who declined participation with characteristics of the Portland sample of 124 dyads revealed that the stroke patient tended to be older, and the couple was more likely to be married in the group of refusers.

Gender data comparing the marital dyad sample and those who declined to participate were also examined. The study sample was made up of a substantially greater percentage of male stroke victims 182\%, compared to $65 \%$ for the refusers), while the refusal group contained a larger proportion (27\%) of male caregivers compared to the marital dyad sample. In looking at the gender data in conjunction with the marital status data, there was a tendency for the group which declined participation to be composed of marital dyads in which the stroke victim was female. In the overall sample, $83 \%$ of the primary respondents (PIs) were female. The fact that a greater percentage of dyads in which the caregiver was male was found in the group which declined, and that such a high percentage of the total group of caregivers was female $(78 \%)$ is in agreement with the 
literature on caregiving roles (Belle, 1982; Brody, 1977; Shanas, 1979) and with the literature indicating a greater willingness among women to participate in survey research.

It was also possible to compare characteristics of the study sample with demographic data from a survey of community hospital - based stroke programs ( CHSP) in oregon during 1979 (Becker et al., in press), and with combined survey data from community hospital-based programs in three states: North Carolina, Oregon, and New York (Becker et al., in press). Table VIII summarizes data on age, gender, and ethnicity distribution among the marital dyad sample, the Oregon CHSP survey, and the three state survey. The study sample is composed of younger stroke patients than either of the two comparison samples or than the National stroke study sample (Weinfeld, 1981) for two apparent reasons. First, all of the comparison samples contained cases which were not initial strokes while this study sample did not. Second, as previously mentioned, excluding those stroke patients who were widowed or single and without an identifiable partner biased the study sample toward a younger age group.

The study sample was composed of a much larger proportion of male stroke patients than the comparison samples. While the National stroke Survey (Weinfeld, 1981), found a $44 \%$ higher incidence of strokes among males, other factors appeared to account for the disproportionate number 
TABLE VIII

COMPARATIVE DEMOGRADYIC DATA EOR MARITAI DYAD SAMPLE, 1979 OREGON CHSP SAMPLE, AND

THREE STATE CHSP SAMPLE

CHARACTERISTIC

$$
\begin{aligned}
& \text { MARITAL } \\
& \text { DYADS } \\
& (N=50)
\end{aligned}
$$

OREGON
CHSP
$(N=1689)$

3 STATE CHSP

$(N=4091)$

$\underline{A G E}$

$$
\bar{X} \text { AGE }
$$

MEDIAN AGE
63.7

63.8
71.9

73.0

GENDER

MALE

$41(82)$

$765(45)$

$1918(47)$

FEMALE

9 (18)

924 (55)

$2173(53)$

\section{ETHNICITY}

WHITE

$47(94)$

$1632(97)$

$3444(84)$

NON WHITE

57 (3)

647 (16)

* Numbers in parentheses are percents. 
of males in the study sample. More than one-third of the subjects in this study were referred from the Veterans Administration Hospital, which serves largely a male population. Also, as previously mentioned, there was a tendency for dyads in which the caregiver, or primary informant was female to be more willing to participate in the study. Furthermore, males are more likely to have living spouses or partners because of the differential longevity of males and females (Cutler \& Harootyan, 1975).

\section{Stroke-Related Data}

Data on the type of stroke and site of lesion were obtained from medical records, including discharge summaries, CT scan reports, and cerebral angiographic reports. All medical records were obtained by having the stroke victim subject or their representative sign a release of medical information form. This form was then submitted to the medical records department of the appropriate hospital or hospitals. Information regarding the type of stroke suffered and the location of the lesion was available for all 50 of the stroke victim subjects. Determination of type and site of the lesion was made from review of the medical records by the investigator, who is experienced in working with stroke patients and in interpreting their medical records. In those cases where the medical records or CT scan reports were unclear or ambiguous as to the type or site of the stroke lesion, interpretation of these data 
was made by the consulting neurologist to the project.

\section{Representativeness of the Sample: Stroke-Related Data}

In this sample, 45 (90\%) of the stroke patients had suffered a thromboembolic stroke, or cerebral infarct, while four (8\%) had suffered a hemorrhagic stroke, and one (2\%) had suffered a lacunar infarct. With regard to the location of the lesion, 30 subjects (60\%) had experienced left hemisphere strokes, 15 (30\%) were found to have had right hemisphere strokes, and five (10\%) had suffered brain stem strokes. The distribution of types of strokes in this sample is in agreement with the 1979 CHSP data (Becker et al., in press), and with data from the National survey of Stroke (Weinfeld, 1981). With regard to site of lesion, the study sample has a greater than expected proportion of patients with left hemisphere lesions. This may in part be due to the fact that a large number of referrals came from speech pathologists, who would be more likely to be working with persons with left hemisphere strokes. It may also reflect the possibility that a greater percentage of those who declined to participate were individuals with right hemisphere strokes.

DATA COLLECTION

\section{Subject Recruitment}

The study sample was obtained through extensive preliminary efforts involved in setting up a referral system 
with seven of the largest area hospitals within the geographical boundaries of the study. These included six major hospitals in the Portland metropolitan area (Emanuel, Good Samaritan, Oregon Health Sciences University, Providence, St. Vincent, and the Veterans Administration medical centers) as well as Salem Memorial-Salem General Hospital in Salem, Oregon. Tables IX and $X$ summarize the sources of referrals. The majority of referrals (92\%) came from facilities having comprehensive rehabilitation centers affiliated with their institutions. In each of the referring institutions, the investigator identified one or two key persons who acted as contact individuals, and who made the initial approach to patients and families to inform them about the study. When potential subjects were willing to learn more about the investigation, the contact person sent a brief referral form to the study office in prepaid envelopes provided. Potential subjects were then contacted by an interviewer, the nature of the project explained in more detail to them, and informed consent obtained in writing if the family and/or patient indicated willingness to participate. In all cases, care was taken to obtain the consent of the attending physician before potential subjects were approached.

Several other recruiting efforts were initiated in order to increase the number of referrals. These included contacts with home health agencies, private physicians 
TABLE IX

TOTAL NUMBER AND PERCENT OF REFERRALS BY REFERRAL SOURCE, PORTLAND STRORE STUDY $(N=210)$

\begin{tabular}{|c|c|c|c|c|c|c|}
\hline REFERRAL SOURCE & $\begin{array}{l}\text { \# IN } \\
\text { STUDY }\end{array}$ & $\begin{array}{r}\# \\
\text { IN }\end{array}$ & $\begin{array}{l}\text { NOT } \\
\text { STUDY }\end{array}$ & & $\begin{array}{l}\text { TOTAL } \\
\text { REFERR. }\end{array}$ & $\stackrel{\$}{\text { TOTAL }}$ \\
\hline V. A. Hospital & 46 & & 36 & & 82 & 39 \\
\hline Emanuel Hospital & 40 & & 23 & & 63 & 30 \\
\hline Salem General Hosp. & 14 & & 9 & & 23 & 11 \\
\hline Good Samaritan Hosp. & 9 & & 11 & & 20 & 10 \\
\hline Providence Hospital & 8 & & 2 & & 10 & 5 \\
\hline OHSU Hospital & 1 & & 2 & & 3 & 1 \\
\hline Other Sources * & 6 & & 3 & & 9 & 4 \\
\hline Totals & 124 & & 86 & & 210 & 100 \\
\hline $\begin{array}{l}\text { * Other referral } \\
\text { physician (2); speech } \\
(2) ; \text { friend }(1) \text {. }\end{array}$ & $\begin{array}{l}\text { sources } \\
\text { patholog }\end{array}$ & & $\begin{array}{l}\text { ude: } \\
(2) \text {; }\end{array}$ & $\begin{array}{l}\text { self } \\
\text { home }\end{array}$ & $\begin{array}{l}(2) i \\
\text { health }\end{array}$ & $\begin{array}{r}\text { private } \\
\text { agency }\end{array}$ \\
\hline
\end{tabular}

Total referrals

Total excluded

Total eligible referrals 166 (79\%)

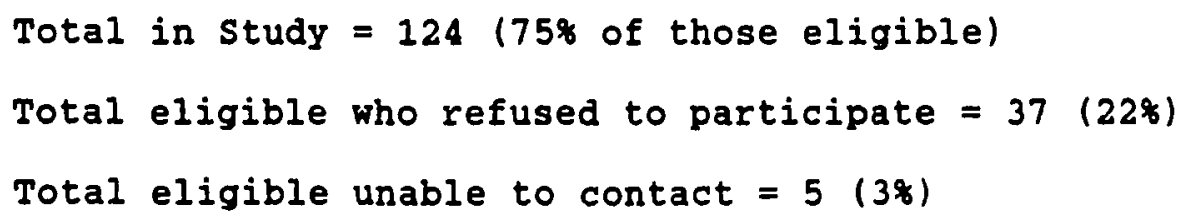


TABLE $X$

\begin{abstract}
NUMBER AND PERCENT OF REFERRALS BŸ SOÜRCE OF REFERRAL, MARITAL DYAD SAMPLE
\end{abstract}

\begin{tabular}{|c|c|c|}
\hline REFERRAL SOURCE & $\begin{array}{l}\text { NUMBER OF } \\
\text { REFERRALS }\end{array}$ & $\begin{array}{l}\text { \% OF TOTAL } \\
\text { REFERRALS }\end{array}$ \\
\hline $\begin{array}{l}\text { Veterans Administration } \\
\text { Med. Center, Portland }\end{array}$ & 18 & 36 \\
\hline Emanuel Hospital, Portland & 13 & 26 \\
\hline $\begin{array}{l}\text { Salem Memorial-Salem General } \\
\text { Hospital, Salem }\end{array}$ & 8 & 16 \\
\hline $\begin{array}{l}\text { Good Samaritan Hospital \& } \\
\text { Med. Center, Portland }\end{array}$ & 4 & 8 \\
\hline $\begin{array}{l}\text { Providence Medical Center, } \\
\text { Portland }\end{array}$ & 3 & 6 \\
\hline $\begin{array}{l}\text { Other (Self, friend, small } \\
\text { community hospital) }\end{array}$ & 3 & 6 \\
\hline $\begin{array}{l}\text { Oregon Health Sciences Uni- } \\
\text { versity Hospital, Portland }\end{array}$ & 1 & 2 \\
\hline Totals & 50 & 100 \\
\hline
\end{tabular}


(internists, neurologists, and physiatrists), stroke clubs, and senior centers. In some hospitals, permission was obtained for project staff to screen the charts of persons admitted or discharged with the diagnosis of stroke, with approval of the attending physician.

\section{Conduct of the Interviews}

Data analyzed in this investigation were collected over a 21 month period from November, 1983 through July, 1985. Once subjects had agreed to participate in the study, every attempt was made to complete the initial interview within three to six weeks following the stroke event. So that a number of potential participants would not be excluded, however, the time between occurrence of the stroke and initial interview was extended to eight weeks in some cases. Most interviews were conducted in subjects' homes (82\% for the primary informants, and $81 \%$ for the stroke victims who could be interviewed), with the remaining interviews being conducted in the hospital setting or in the stroke project office if this was more convenient for the subject. Interviews were carried out by three experienced interviewers, including the investigator. All of the interviewers had extensive experience in counseling and interviewing persons and families in crisis situations. Every attempt was made within the practical constraints of data collection to have the same interviewer complete both the initial and followup interviews with a particular 
subject.

\section{HUMAN SUBJECTS ASSURANCES AND CONCERNS}

During all phases of the project, care was taken to assure that subjects' rights to privacy and anonymity were protected. Subjects were informed both verbally and in writing that the information they provided would be treated as group data only, and that they would not personally be identified in any of the reported results. Interview protocols were assigned code numbers for identification purposes, and completed interviews as well as the identification code book were stored in separate locked files in the stroke study office.

In addition to the procedures adopted to insure the subjects anonymity and privacy, care was taken to inform subjects verbally and in writing that they could decline to participate in the study or withdraw from the study at any time without jeopardizing the stroke victim's rehabilitation program, future hospital and medical care, or related benefits. These written and spoken assurances were provided at the time subjects were initially informed about the study, and before each interview began. In addition, special efforts were made to confirm that the stroke victim subjects understood both the nature of the study and their rights as potential subjects. Typical and frequent comments such as "If it will help other stroke patients and their 
families, I'd be happy to participate" helped reassure the interviewers that stroke victim subjects were giving informed consent.

Because of the length of the interview lapproximately one and a half hours), the sensitive nature of some of the questions, and the timing of the interview relative to the stroke event itself, care was taken by the interviewers to be sensitive to signs of fatigue or discomfort on the part of the subjects, and to assure subjects that they could take a rest break, or decline to answer certain questions if they chose.

\section{DATA COLLECTION INSTRUMENTS}

\section{Stroke Survey: Social Support and Well-Being}

Data were collected primarily through the use of a questionnaire designed for the study, the stroke survey: Social support and Well-Being. As the instrument was developed, it became necessary to actually create three separate versions of the questionnaire: one for use with the caregivers, one to be administered to stroke-victim subjects, and a third version for caregivers responding for stroke victims who were unable to respond for themselves. In addition, a follow-up version of the protocol was developed for administration at Time 2 , six months after the initial interview. Two forms of the follow-up interview were developed, one to be given to primary informants and to 
stroke victims who could participate in the interview, and a second Form for primary informants providing information about the stroke victim. Having these targeted versions of the protocol improved the flow of the interview, and made the instrument less cumbersome. Copies of the interview protocols for Time 1 and Time 2 are found in Appendix $A$ and Appendix B.

The interview took approximately an hour and a half to complete in its final form. The instrument was pretested on six families who were not part of the study sample, and shortened considerably from its original version.

The following are the major types of data collected with the interview protocol:

Demographic. Information was obtained regarding age, sex, race, marital status, household, children, pets, education, employment, income, forms of transportation utilized, and residential characteristics.

Group Memberships / Recreational Activities / Community services. Information regarding the types and numbers of organizations to which subjects belonged, including religious affiliation, and frequency of participation in these organizations was obtained. Respondents were asked about the importance of religion in their lives. They were also asked to describe their involvement in volunteer activities, and the frequency of participation in volunteer activities. Information 
regarding types and numbers of recreational activities and hobbies engaged in by respondents was also gathered. Subjects' use of community services and their satisfaction with these services was determined.

Health Status. Questions regarding health status at the time of the initial interview sought to establish a baseline of self-perceived health prior to the stroke event. At Time 2, similar questions sought information about present health status. Subjects were asked to rate their overall health status, to compare their present health status to what it was six months prior to the stroke, and to predict overall health status six months into the future. other health-related questions probed for the occurrence of positive and negative life events in the year before the stroke and between the Time 1 and Time 2 interviews, the existence of chronic health problems and disabilities, number of hospitalizations in the year prior to the stroke and between interview times, number of physician visits within the same time period, number of regular medications before and since the stroke occurred, and the presence of any emotional problems for which the respondent had desired or sought help. Questions were also asked regarding appetite changes or sleep disturbances in the six months prior to the stroke, and in the time period between interviews. 
Perceptions and Feelings Regarding the stroke. Subjects were asked questions about their perceptions of what they might have done to prevent the stroke, their concerns about the possibility of another stroke, their concerns about future care related to the stroke, and to rate the overall severity of the patient's stroke-related physical problems in terms of the amount of assistance he or she required. Other questions related to the stroke event included inquiries about sources of strength and support in dealing with the stroke, and overall satisfaction with the health care received for the stroke. Respondents were asked whether they had previously known anyone who had experienced a stroke, and whether or not and to whom they ascribed "blame" for the stroke occurring. At Time 2, additional questions were incorporated which probed caregivers' perceptions of personality and behavioral changes in the stroke victim, and the degree of felt burden experienced by the caregiver. At this time questions also were asked about participation in support groups such as stroke clubs, and the perceived helpfulness of these groups. Finaliy, the Time 2 interview included open-ended questions about major worries and concerns related to the stroke, about types of support that had been helpful, or that the respondent desired but did not find available, and about present kinds of assistance desired.

Spousal Relationship. In order to characterize the 
quality of the spousal relationship (which was defined as married or iiving togetiner as married), several questions focused on the frequency of joint activities, manner of dealing with potential areas of marital conflict 1 finances, major decisions, handling children and in-laws, etc.l, and who in the relationship held major responsibility for managing family finances, making major decisions, and completing household tasks. Subjects were asked to assess the likelihood that they and their spouse or partner would confide in one another, to rate their overall satisfaction with the marital relationship, and to compare their relationship to that of other couples they've known.

Social Network/Support. Because it was the major focus of this study, detailed information was obtained about the social networks of both stroke victims and their partners. Using a method developed by Fischer and his colleagues (Fischer, 1982), first a series of questions about various types of support the respondent received from his or social network (instrumental, emotional/affective, informational/cognitive, and companionship) served to elicit a list of names of support persons, up to 20 total names. Descriptive information was obtained about each of these support persons, including gender, relationship to the respondent, and type of support provided. After the respondent selected up to ten of the support persons named previously as being part of the individual's close personal 
network, further descriptive information was obtained about these intimate sociai network members inciuding age, residence location, duration of the relationship, frequency of contact, and degree of reciprocity in the relationship. Global assessments of network functioning included questions about the respondent's overall satisfaction with the quantity and quality of social contact, and measures of network density and degree of interconnectedness. From the perspective that not all network relationships are supportive, and that some network members may create additional stress for the focal person, subjects were also asked whether, during the time of the acute stroke event and at the time of follow-up, some network members had helped less than expected or not at all; whether there were individuals who tried to be helpful but made the situation worse; whether some network members were perceived as "out to make life difficult" for the respondent; and whether there were persons with whom the subject didn't get along.

\section{Other Measures}

Along with the questionnaires developed for the study, three established measures of psychological well-being, a caregiver burden scale, and two measures of level of functioning in activities of daily living were employed. Reliability of each of these measures with this sample was determined by computing Cronbach's alpha, a measure of internal consistency (Cronbach, 1951). These instruments 
are described in the following sections.

Index of Psychological Well-Being (IPWB). This is an eight item, self-report scale designed to measure mental health in a general population (Berkman, 1971). It is composed of both positive and negative feeling-state items, and describes the relative strength of an individual's positive and negative feelings, rather than an absolute amount of one or the other. Separate scores are computed for negative and positive feelings, and combined on a matrix to form a total (balance) score of psychological well-being. For example, an individual with a negative feelings score of three and a positive feelings score of eight receives a balance score of two on the scale. Table XI illustrates the scoring system of the IPWB. This method of measuring psychological well-being helps to explain why some people who seem to have a very high number of negative forces acting on them are still able to maintain a sanse of wellbeing, while others who appear to be exposed to only a small number of negative experiences become extremely depressed.

The items in this scale come from those used by Bradburn and Caplovitz (1965) to measure psychological wellbeing in their studies of happiness. For purposes of this study, the change adopted by Schulz and Decker (1982) in their study of spinal cord injured persons for one item on the Index (from "so restless you couldn't sit long in a chair" to "so restless you had to move about") was 
TABLE XI

SCORING MATRIX FOR THE INDEX OF PSYCHOLOGICAL WELL-BEING

\begin{tabular}{ccccc} 
Negative & \multicolumn{4}{c}{ Positive Feelings Score } \\
Feelings Score & $7-9$ & $4-6$ & 3 & $0-2$ \\
$0-1$ & 1 & 2 & 3 & 4 \\
$2-3$ & 2 & 3 & 4 & 5 \\
$4-5$ & 3 & 4 & 5 & 6 \\
$6-15$ & 4 & 5 & 6 & 7
\end{tabular}

maintained in this study, since some of the stroke-victim subjects were unable to walk. Berkman (1971) cites a parallel association between scores on the Index of Psychological Well-Being and mental health/life stress ratings by psychiatrists in the Midtown Manhattan study (Langner and Michael, 1963) as an indication of criterionoriented concurrent validity. Reliability of the IPWB for this sample was considered acceptable. Alpha levels ranged from .84 for PI positive well-being to .45 for stroke victim positive well-being.

Center for Epidemiologic Studies - Depression Scale (CES-D). This is a 28 item version of a self-report scale designed to measure depressive symptoms in the general population (Radloff, 1977). The items were selected from previously validated longer scales: Beck Depression Inventory (Beck, Ward, Mendelson, Mock, \& Erbaugh, 1961); Zung's Self-Rating Scale (Zung \& Durham, 1965); MMPI- 
Depression Scale. The CES-D is composed of four factors interpreted as depressed affect, positive affect, somatic and retarded activity, and an interpersonal factor. The CES-D Scale was designed to avoid the problem characteristic of some depression scales of placing too much emphasis on somatic items which frequently characterize non-depressed older persons. Radloff (1977) reports that the internal consistency, test-retest reliability, and validity of the scale are high and correlations between the CES-D and age, social class, and gender are minimal. Content validity was established based on the clinical relevance of symptoms which comprise the items of the scale. Criterion-oriented validity was also established by correlations with other valid self-report depression scales (Bradburn, 1969, $\underline{r}=$ .61 ; Langner, 1962, $\underline{\underline{x}}=.54$; and Lubin, 1967, $\underline{\underline{x}}=.51$ ) and with clinical ratings of severity of depression $(\underline{x}=.56)$. Construct validity was established by demonstrating a pattern of relationships with other variables. Reliability of the scale was established through test-retest procedures $(\underline{r}=.54)$ and analysis of internal consistency (Cronbach's alpha $=.85 ;$ split halves $r=.771$. The CES-D is not designed to be used as a clinical diagnostic tool, but is felt to be most useful in identifying groups at risk of depression. Cronbach's alphas for the CES-D in the present sample were very high lalpha $=.89$ for the caregivers, and .86 for the stroke victim respondents). 
The Life-orientation Test (LOT). This is an eight item scale developed by Scheier and Carver (1983) to measure dispositional optimism, which they define in terms of the favorability of the general outcome expectancy that a person has come to hold. Rather than viewing optimism as a construct representing a transient state, and as an outcome variable, these authors have developed a model, based on studies using this scale, which conceptualizes optimism as a stable personality characteristic having important implications for the manner in which people manage their actions, leading in turn to other important consequences. For this study, we were interested in exploring the relationship between dispositional optimism, as expressed in responses to this scale, and several outcome measures, including symptom reporting on the IPWB and the CES-D, the degree of felt burden expressed by primary informants in caring for the stroke patient, and perceptions and feelings about the stroke itself. Initial evidence for convergent validity of the LOT was reported by scheier and Carver (1983) who found the LOT to correlate significantly in the expected direction with other measures, such as a measure of locus of control (Rotter, 1966), Rosenberg's self-esteem scale (Rosenberg, 1965), a hopelessness scale (Beck, Weissman, Lester, and Trexler, 1974), Beck's Depression Inventory (Beck, 1967), a measure of perceived stress (Cohen, Ramarck, and Mermelstein, 1983), a measure of social 
desirability (Crowne and Marlowe, 1964), the Self-Consciousness Scale (Fenigstein, Scheier, and Buss, 1975), and a test of alienation (Maddi, Robasa, and Hoover, 1979). Evidence for the discriminant validity of the LOT is found in the fact that it correlates only modestly with the other measures described above. The authors argue that this indicates that the LOT is not completely redundant of the other measures with which it was compared in establishing its psychometric properties.

With regard to internal consistency, Cronbach's alpha for the entire 8-item scale was found to be.76, indicating an acceptable level of internal consistency. Test-retest reliability as indicated by a test-retest correlation of .79 , is indicative of the measure's stability over time. For this study sample, Cronbach's alphas were acceptable (alpha $=.65$ for caregivers and .61 for stroke patients) .

Caregiver Burden Scale. This measure consists of a 14 item Likert-type scale adopted from a measure described by Zarit, Reever, and Bach-Peterson (1980) for use with the caregivers of elderly demented persons. For use in this study it was necessary to reword some of the items, and to eliminate others which appeared inappropriate for use with a stroke sample. The psychometric properties of the Caregiver Burden Scale described by zarit et al. have been established, with internal consistency reported to be high (alpha $=.85)$ (Zarit, personal communication, October 11, 
1985). The instrument appears to have content validity, and the internal consistency of the scale with this study sample was very high (alpha $=.85)$. The Caregiver Burden Scale was administered to caregiver subjects only, and only at Time 2 . Barthel Index. This instrument is a widely used, easily scored index of functional independence in activities of daily living (Mahoney \& Barthel, 1965) whose reliability and validity have been supported in a number of studies (Donaldson, Wagner, and Gresham, 1973; Wylie, 1967). It provides a simple, yet reasonably sensitive method for evaluating the physical functioning of a disabled individual at a given time, and for assessing change in physical functioning over time. This index assigns $0,5,10$, or 15 points to the level of performance on each of 10 activities of daily living, with a maximun obtainable score of 100 when all activities are carried out completely independently. At each interview period, stroke-victim subjects rated themselves on the Barthel Index, and the primary informants independently rated the stroke victim's level of functional independence with the same instrument. Internal consistency of the Barthel for the present study sample was very acceptable lalpha $=.90$ for primary informants, and .81 for stroke victims.)

Adaptive/Social Functioning Scale (ASF). This is a nine item scale of selected activities thought to reflect adaptive and social functioning following brain injury. It 
was adopted from the instrument used by the Comprehensive stroke Center of oregon in a 1979 survey of stroke and rehabilitation outcomes. The scale rates individuals as to whether they are able to perform the described activity since the stroke, whether they were capable of performing the activity before the stroke but not since, or whether they were incapable of performing the activity in question before the stroke. Four of the items (those related to using the telephone, using public or private transportation, performing general outdoor activities and engaging in formal and informal social activities) are rated as to whether they are performed independently or with assistance. No information about the psychometric properties of this scale is available although the results of one study using the ASF are available (Becker et al., in press). For caregivers in this sample, internal consistency of the ASF scale was high $($ alpha $=.87)$.

\section{Medical Records Data}

In addition to information obtained from the interview schedule and from the scales described in the previous section, certain information related to the stroke was obtained from the stroke patient subjects' medical records. This included data on the type of stroke, site of lesion, length of hospitalization, and types of therapy provided. 
OPERATIONAL DEFINITIONS

Demographics

Age. Age was defined in this study as the subject's age in years on the day of the initial interview.

Household Composition. This was coded as: spousal couple only, children living in the home, or "other".

Education. Amount of education was the highest reported level of education completed, on an eight-point scale from (1) less than seven years of school to (8) graduate or professional training.

Employment Status. Subjects defined their employment status as: never worked outside the home, employed fulltime, employed part-time, unemployed, retired, or other. At the time of the first interview, employment was defined as pre-stroke employment status.

Income. Income was defined as total household income in the year prior to the stroke. Subjects were asked to indicate their income category on a scale from (1) less than $\$ 5,000$ a year to (9) more than $\$ 50,000$ a year.

\section{Religiosity}

Religiosity was defined as a subject's summed score on a three-item index (RELIG) which included: the reported importance of religion to the respondent, on a 5 point scale from (1) very unimportant to (5) very important; reported level of religious activity, from (1) inactive to (4) very 
active; and frequency of attendance at religious activities or services, from (0) never to (5) greater than weekly. Cronbach's alphas for this index were .80 for the caregivers and .70 for the stroke victims. Alpha levels were improved to .87 for the caregivers and to .80 for the patients if the item regarding the importance of religion was deleted from the index.

Orqanizational Activities and Memberships

Organizational Memberships. This was a computed total of all the organizations to which the subject indicated he or she belonged. Type of organization was also coded for descriptive analysis.

Volunteer organizations. This was a computed total of the number of groups or organizations to which the respondent reported giving volunteer time.

\section{Self-Reported Health status}

Objective Health Status. Objective health status was defined as the subject's summed score on a four-item index (OBHEALTH) which included: the number of limiting disabilities the individual reported, the number of chronic illnesses, the number of visits to the doctor during the previous six months, and the number of medications they were currently taking. Cronbach's alphas for this index were .76 for the partners and .55 for the stroke patients.

Subjective Health. Attempts were made to construct an 
index of subjective or perceived health which included individuais' overall assessment of their present health status; whether their health had gotten better, stayed the same, or was worse compared to six months ago; and whether they expected their overall health to change within the next six months. These efforts did not result in a subjective health index with an acceptable level of internal consistency. Therefore, the item related to overall assessment of present health status (HEALTH) was chosen as the best indicator of subjective health for later analyses.

\section{Stressful Life Events}

Two separate scores were computed for major life events which subjects reported in the year prior to the stroke and during the six months between interviews: A score for positive life events and a score for negative life events. If there was ambiguity in interpreting the event as positive or negative, the subject was asked how he or she perceived the event.

\section{Stroke-Related Factors}

Severity of the stroke. Severity of the stroke disability in this study was defined as the level of assistance required or utilized by the stroke victim in accomplishing activities of daily living and in dealing with various cognitive tasks. The subject's scores on two measures of functional independence were used to define the 
severity of stroke-related disability. The Barthel Index (Mahoney \& Barthel, 1965) is a measure oi functional independence in accomplishing physical activities of daily living (ADL's) such as feeding, dressing, grooming, ambulating, and toileting. The Adaptive/Social Functioning Scale (ASF) is an activity scale which quantifies level of independence in such activities as communication, social interaction, ability to handle money, and ability to use public or private transportation.

Type of stroke. Strokes were categorized as (1) thromboembolic (infarction): (2) hemorrhagic; or (3) lacunar. Cerebral infarctions are types of strokes caused by either a clot (embolus) traveling from another part of the body to block the flow of blood to the brain, or by the narrowing of a blood vessel wall related to atherosclerotic plaque (thrombi) restricting the flow of blood to a critical area of the brain. While some studies differentiate between thrombotic and embolic strokes, and between types of embolic strokes, the consulting neurologist on this study recommended that we consider thrombotic and embolic infarctions in one category of thromboembolic events. This is because it is difficult to determine in many cases whether a cerebral infarction is caused by a thrombus or an embolus. Hemorrhagic strokes are those caused by the bleeding of one or more blood vessels within the brain, with the resultant death of brain tissue. Lacunar strokes are 
generally mild events, related to small hemorrhages within the brain. Strokes of the hemorrhage type are usually related to hypertension.

Site of Lesion. The site of lesion refers to whether the stroke occurred in one or the other cerebral hemisphere, in the brain stem, or elsewhere in the central nervous system (e.g., a spinal stroke). Strokes were classified in this study as having occurred in the left cerebral hemisphere, the right cerebral hemisphere, the brain stem, or elsewhere (the "other" category). Site of lesion was determined by medical records, especially hospital discharge summaries and CT scan reports.

First, Completed stroke. It was important to determine for purposes of this study, that stroke victim subjects had not suffered previous, completed stroke events that would have perhaps affected the social support system of the subject or the primary informant, or their psychological well-being. Previous transient ischemic attacks (TIA's) or completely resolved ischemic neurologic deficits (RIND's) did not exclude potential subjects from inclusion in the study. Because some CT scan reports on stroke victim subjects indicated previous small lesions for which the subject had never been hospitalized, for which there was no clinical medical documentation, and of which the subjects themselves were unaware, the final determination as to whether a potential subject should be excluded because of a 
previous completed stroke was made by the project's consulting neurologist on the basis of medical records and CT scan reports.

Stroke-Related Personality and Behavioral Changes. A list of 19 behaviors or personality traits, six with positive connotations and 13 with negative implications, was prepared, based on the relevant stroke literature and the investigator's experience with stroke families. Partners were asked at the time of the second interview to indicate whether the stroke patient exhibited less, about the same degree, or more of each of the behaviors since the occurrence of the stroke. From this information, five scores were computed: the total number of negative behaviors which had increased in frequency; total number of positive behaviors which had increased; total number of negative behaviors which had decreased, total number of positive behaviors which had decreased, and total number of behaviors which had remained about the same.

\section{Perceptions of the stroke}

Attempts to create an index of stroke perceptions which included scores on questions regarding concern about another stroke, concern about future care, and overall stroke severity did not result in acceptable alpha levels. Therefore, responses to these questions were retained as individual items for later analyses. 
Quality of the Stroke Victim - Partner Relationship

Several indices were created from questions which probed various aspects of the stroke victim - caregiver relationship. These indices are described below.

Index of Marital Satisfaction. This was a four-item index (MARR4) which summed respondents' scores on items dealing with: the probability that they would discuss an important problem with their partner, from (1) very unlikely to (5) very likely; the likelihood that the partner would discuss a serious problem with them; perceived level of happiness in the relationship, from (1) very unhappy to very happy; and how they would compare their relationship to other couples they've known, from (1) worse to (3) better. Alpha levels for this index were .70 for the partners and .48 for the patients.

Index of Marital Disagreement. Marital disagreement was operationalized as the total score a subject received on a five-item index which assessed frequency of disagreement, from (1) rarely to (3) frequently, with regard to finances, children, major decisions, sharing of household tasks, and leisure time activities. Alpha levels for the index were .61 for the caregivers and .69 for the stroke victims.

Index of Shared Marital Activity. Shared marital activity was defined for purposes of the study as a subject's total score on a six-item index which assessed frequency of shared or joint activity involving: outside 
interests or activities, conversations about ideas or opinions, laughing together, calmly discussing something, working together on a project, and number of joint organizational memberships. Individual item scores for the first five items could range from (0) never to (6) more often than once daily. Total number of joint organizational memberships was computed as part of the Index score. Alpha coefficients for this index were at acceptable levels: alpha $=.66$ for the caregivers, and alpha $=.73$ for the patients.

\section{Social Activity}

A five-item index of social activity (SOCIAL) was created by summing subjects' scores on items which assessed the frequency with which they had people over to their home for a meal, went to others' homes for a meal, had visitors, went visiting in someone's home, and went out with other people. These items were adopted from Fischer's (1982) northern California survey. Alpha coefficients for this index were quite high -.84 for the partners, and .87 for the stroke patients.

\section{Social Network}

The concept of individual, or ego-centered networks of other persons to whom the subject has ties of kinship, friendship, neighborly relationship, or work relationship has been frequently used as one measure or indicator of 
social support. The individual social networks of subjects in this study was derined as comprised or those individuals, up to a total number of 20 , whom the subject named in response to a variety of questions about the people in his or her life who provide instrumental, informational, or emotional support, or with whom the subject spends time socializing. The intimate, or close social networks of subjects included up to 10 persons from the total network list whom the subject indicated were especially important to him or her, or to whom the subject felt most close. The method used to elicit names of network members was modelled after that suggested by Fischer (1982).

Structural Network Measures. Structural network measures are those characteristics of the network that describe its structural, or quantitative aspects. These include the total number of persons in the network, frequency of contact with individual network members, ages of network members, the length of time the subject has known the network member, and the nature of the relationship (parent, child, spouse, other kin, friend, neighbor, work associate). Other structural network measures utilized in this study included two indicators of the interconnectedness among network members (density and degree), as well as a measure of the overlap in network membership between the networks of the stroke victim and the primary informant (boundary density). 
Prestroke social network. This was defined as the total number of persons named by the respondent in response to specific questions about network activities before the stroke.

Stroke period social network. This network included those persons named by the subject in response to specific questions about network members who provided help and support, or who were less than helpful during the period of time between the onset of the stroke and the initial interview.

Poststroke social network. This network included those individuals named by the subject in response to network eliciting questions at Time 2 .

Frequency of network contact. This measure included summed frequency counts of both face-to-face contacts with non-household network members, and phone and letter contacts. In addition, a combined score of the frequency of boch types of contacts was computed. Frequency of contact was determined for members of the intimate network only, and a mean frequency of contact score for a subject was determined by summing and averaging across all members of the intimate network.

Network density. Network density is a measure of the interconnectedness among the members of a social network. It is defined as the number of actual network ties which exist relative to the number of possible ties which could 
exist. In this study, the existence of a tie between two network members was determined by asking the question, "Does $X$ know Y?" The formula,

$$
\text { Density } \frac{100 \times N A}{N(N-1) / 2}
$$

where $N A$ equals the actual number of network ties, and $N$ equals the number of network members, was used in this study to determine network density.

Network degree. Degree is also a measure of network interconnectedness which corrects for differences in network size across subjects. It is conceptualized as the average number of ties per person in a given network, and is mathematically defined as:

$$
\text { Degree }=\frac{2 \times N A}{N}
$$

where $N A=$ the number of actual ties and $N=$ the number of persons in the network.

\section{Social support}

Social support is defined as comprised of those mechanisms whereby social network relationships promote positive outcomes, or serve as a buffer against negative outcomes, especially in assisting an individual or family to cope with stressful life events. Social support was conceptualized in this study as of four types: instrumental, affective or emotional, informational/ 
cognitive, and companionship. That is, individual network members were categorized as providing one or more of the types of support described below.

Instrumental support. Instrumental support was defined as the provision of tangible aid, such as financial assistance, transportation, or assistance with household chores or activities of daily living.

Affective support. Affective support has been defined as the communication of direct, positive affect; for example, feedback that one is loved, respected, confided in, and "belongs". It is also described as emotional support. In this study, confidant support was used as the primary measure of emotional support. Expressed or perceived understanding of the situation of the caregiver and of the patient was an additional measure of emotional support. Informational (cognitive) support. This type of support is defined as the communication of information that helps the individual negotiate his or her world. The information provided may range from the very specific, such as how to deal with various aspects of the stroke, or how to locate a stroke club support group, to more subtle types of information which enable the individual to appraise the appropriateness of feelings, beliefs, attitudes, and goals. Informational support was defined in this study as the number of network members who provided information and advice, or from whom the respondent would seek information 
and advice.

Companionship support. This was measured by questions which asked respondents to indicate those persons with whom they spent time socializing, or "just having an enjoyable time together", and those persons with whom they enjoyed sharing interests or hobbies.

Reciprocity. Reciprocity in network exchanges has been consistently found to be an important aspect of social support (Antonucci \& Depner, 1982; Chapman, Pancoast, \& Parker, 1983; Gottlieb, 1981; Kahn \& Antonucci, 1980; Roberto \& Scott, 1984; Wentowski, 1981). In this study, reciprocity was determined by computing the number of network members with whom the subject indicated they maintained or would expect reciprocal exchanges in the areas of task-oriented support, assistance when ill, confiding, and providing information and advice. A total reciprocity score was then computed for each type of supportive exchange. This allowed for determination of those subjects who reported a reciprocal balance in their supportive relationships versus those who appeared to be "underhelped" or "overhelped" (Roberto \& Scott, 1984).

\section{Psychological Well-Being}

In this study, three measures of psychological wellbeing were employed: the Index of Psychological Well-Being (Berkman, 1971), the Center for Epidemiologic studies Depression Scale (Radloff, 1977), and the Level of Optimism 
Scale (Scheier \& Carver, 1983). At Time 2, a Caregiver Burden Scale (zarit et al., 1980) was administered to the primary informant subjects as an additional indicator of subjective well-being. Well-being was defined multidimensionally in this study, based on subjects' scores on these measures. The relationships among the various measures were also explored, and will be reported in the following chapters.

\section{DATA ANALYSIS PROCEDURES}

The initial steps in data analysis involved the development of codebooks for both the initial interview and follow-up interview data, the coding of raw data onto Fortran coding forms, the keypunching of data onto cards, and the storing of data on a permanent computer tape.

Extensive quantitative and qualitative analyses of the data collected in this study were carried out. The first stage of data analysis involved obtaining and analyzing frequencies and descriptive statistics on the sample. This was followed by the computation of numerous pearson correlation coefficients to obtain an overall picture of which demographic, perceived health, stroke-related, and social network variables correlated with each other and with the outcome measures of psychological well-being and depression.

The next stage of data analysis involved the 
completion of reliability analyses of indexes constructed to measure health, religiosity, social actiuities, marital quality, and caregiver burden. Reliability analyses for this sample were also carried out on the activities of daily living, psychological well-being, depression, and optimism measures.

A fourth stage of data analysis involved examining the Time 1 and Time 2 data with regard to certain variables of theoretical interest in this study for evidence of change over time, utilizing both distribution of change scores and t-tests. For example, the size and density of the social network at Time 1 and Time 2 were examined to look for changes in the predicted direction of smaller network size and increased network density.

The final stage of data analysis involved the use of hierarchical multiple linear regression analyses and changefocused multiple regression analyses to determine to what degree subjective well-being could be predicted from demographic, social network, social support, perceived health, and stroke-related variables, at each interview time, and accross time. All computer-assisted data analysis was carried out through the use of the statistical Package for the social sciences, 2nd edition (Nie, Hull, Jenkins, Steinbrenner, Bent, 1975), and SPSS Update (Hull \& Nie, 1981).

The following two chapters report results of the 
descriptive analyses of the data. Chapter IV describes demographic and other individual characteristics of the respondents, while Chapter $V$ summarizes the information concerning their social networks and social support. 


\section{DESCRIPTIVE ANALYSIS: THE SAMPLE}

In order to place other findings of the study in context, this chapter describes demographic characteristics of the stroke victims and their partners, their pre-stroke social activities, self-reported health, and perceptions of the stroke. Changes in these aspects at Time 2 are also described. Characteristics of subjects' social networks and social support are discussed in detail in Chapter V.

\section{DEMOGRAPHIC CHARACTERISTICS OF THE SAMPLE}

Demographic data for the sample are summarized in Tables XII (strcke patients), XIII (partners), and XIV (couples). The stroke patient subjects were younger, and more likely to be male than comparable regional and national stroke samples. The sample had a slightly higher pre-stroke annual median household income than the $U$. $S$. population, and they were slightly more educated. These demographic characteristics are discussed in detail in the following sections.

Age

The 50 stroke patients in the sample had a mean age of 63.7 years, S. D. $=9.85$, with a range of 37 to 90 years. Ninety-two percent were over 50 years of age, and $68 \%$ were 60 years or older. Their partners had a mean age of 62.5 
TABLE XII

GENERAL DEMOGRAPHIC DATA: STRORE PATIENTS

$\underline{A G E}$

$\begin{array}{rll}2 \% & 31-40 \text { YEARS } & \text { MEAN }=63.7 \text { YRS. } \\ 6 \% & 41-50 & \text { RANGE }=37-90 \\ 26 \% & 51-60 & \\ 40 \% & 61-70 & \\ 22 \% & 71-80 & \\ 48 & 81-90 & \end{array}$

GENDER

$\begin{array}{ll}18 \% & \text { FEMALE } \\ 82 \% & \text { MALE }\end{array}$

ETHNIC STATUS

$\begin{aligned} 94 \% & \text { CAUCASIAN } \\ 4 \% & \text { BLACR } \\ 2 \% & \text { OTHER }\end{aligned}$

EDUCATION

$\begin{aligned} 24 \% & \text { LESS THAN HIGH SCHOOL GRAD } \\ 28 \% & \text { HIGH SCHOOL GRAD } \\ 24 \% & \text { PARTIAL COLLEGE } \\ 8 \% & \text { COLLEGE GRAD } \\ 16 \% & \text { POST-GRADUATE }\end{aligned}$

\section{PRE-STRORE EMPLOYMENT STATUS}

$\begin{aligned} 36 \% & \text { WORKING FULL TIME } \\ 12 \% & \text { WORKING PART TIME } \\ 4 \% & \text { UNEMPLOYED } \\ 42 \% & \text { RETIRED } \\ 6 \% & \text { HOMEMARER }\end{aligned}$


TABLE XIII

GENERALI DEMOGRAPHIC DATA: CAREGIVERS

$\underline{A G E}$

$\begin{array}{rll}6 \% & 31-40 \text { YEARS } & \text { MEAN }=62.5 \text { YRS } \\ 6 \% & 41-50 & \text { RANGE }=36-84 \\ 30 \% & 51-60 & \\ 36 \% & 61-70 & \\ 18 \% & 71-80 & \\ 48 & 81-90 & \end{array}$

GENDER

$$
\text { 82\% FEMALE }
$$

18\% MALE

ETHNIC STATUS

$\begin{aligned} 92 \% & \text { CAUCASIAN } \\ 4 \% & \text { BLACK } \\ 2 \% & \text { HISPANIC } \\ 2 \% & \text { OTHER }\end{aligned}$

\section{EDUCATION}

$\begin{aligned} 20 \% & \text { LESS THAN HIGH SCHOOL GRAD } \\ 36 \% & \text { HIGH SCHOOL GRAD } \\ 4 \% & \text { TECHNICAL SCHOOI } \\ 26 \% & \text { SOME COLLEGE } \\ 6 \% & \text { COLLEGE GRAD } \\ 8 \% & \text { POST-GRADUATE }\end{aligned}$

\section{PRE-STROKE EMPLOYMENT STATUS}

$\begin{aligned} 22 \% & \text { WORRING FULL TIME } \\ 14 \% & \text { WORR.ING PART TIME } \\ 2 \% & \text { UNEMPLOYED } \\ 34 \% & \text { RETIRED } \\ 28 \% & \text { HOMEMAKER }\end{aligned}$


years, S. D. $=10.7$, with a range of 36 to 84 years. Ninety-two percent of the caregivers were also over 50 years old, and $66 \%$ were over 60 . The stroke patients were younger than both the Oregon Community Hospital Stroke Program sample (Becker et al., in press), and the National Survey of Stroke sample (Weinfeld, 1981). As discussed in Chapter III, possible reasons for this age discrepancy were that only stroke patients with identified partners were included in this sample, and only persons who had suffered a single stroke.

\section{Gender}

Forty-one, or $82 \%$ of the stroke patients were male. This is a higher ratio of male to female stroke victims than is reported in surveys of stroke incidence and prevalence. Specific gender comparisons of this sample and the oregon CHSP and National Survey of Stroke samples were summarized in Chapter III (Table VIII). Reasons for the high proportion of males in this stroke patient sample appear to be: 1) over one-third of the patients in this study were referred from a Veterans Administration Hospital; 2) in this age group men are more likely to be married or to have an intimate partner, a requirement for participation in the study; and 3) there was a tendency for more female patients who were referred to the study and their partners to decline to participate. 
Education

The strcke patients in the sample were comparable in education to the population of the Portland SMSA (U. S. Bureau of the Census, 1983b.) While $24 \%$ of the stroke patients had not completed high school compared to $21 \%$ for the Portland SMSA, $48 \%$ had at least some college, compared to $38 \%$ for the Portland SMSA (National Decision Systems, 1982). This sample of stroke patients was more educated than the national average, where figures indicate that $29 \%$ of the over 25 white population have not completed high school and $33 \%$ have some college education (U. S. Bureau of the Census, 1983a).

Spouses were also comparable in education to the Portland SMSA population and more educated than the national average. Twenty percent had less than a high school education, while $40 \%$ had at least some college.

Pre-stroke Employment status

Prior to the stroke, $36 \%$ of stroke patients were working full-time and $12 \%$ were working part-time outside the home. Almost half (42\%) described themselves as retired, and $6 \%$ weze homemakers. Twenty-two percent of the spouses were employed full-time and $14 \%$ were employed part-time. Twenty-eight percent described themselves as homemakers, and $34 \%$ were retired. 
Stroke Patient - Partner Relationship

This sample was characterized by long-term, stable partner relationships. Couples had known each other a mean of 34.9 years, with a range of 6 to 63 years. Only $10 \%$ of the couples had known one another less than 10 years, while $70 \%$ had been acquainted more than 30 years. For those couples who were married, mean length of time married was 32.07 years, with a range of 1 to 58 years. Seventy-five percent had been married more than 20 years.

\section{Income}

The sample had a pre-stroke mean annual household income of $\$ 19,440$ and a median income of $\$ 18,375$. This median income is higher than the $\$ 16,982$ national median income for all households (U.S. Bureau of the Census, 1983a).

\section{Residential Setting}

Most of the sample lived in suburban areas (54\%), while $34 \%$ lived in urban settings, and $12 \%$ in rural areas. Seventy-two percent lived in single family homes, and $74 \%$ owned their residences. In terms of potential availability of social support, subjects were long-time residents of their area. Both stroke patients and spouses had lived in the area a mean of 29 years.

Household Make-Up

Seventy-four percent of the sample lived in two-person 
TABLE XIV

GENERAL DEMOGRAPHIC DATA: DYADS

RELATIONSHIP
$92 \%$
MARRIED
$8 \%$
LIVING AS MARRIED

\section{LENGTH OF RELATIONSHIP}

$\begin{array}{rl}10 \% & \text { LESS THAN } 10 \text { YEARS } \\ 12 \% & 10 \text { TO } 20 \\ 8 \% & 21 \text { TO } 30 \\ 70 \% & \text { MORE THAN } 30 \text { YEARS }\end{array}$

PRE-STRORE HOUSEHOLD INCOME

$\begin{array}{rl}2 \% & \text { LESS THAN } \$ 5,000 / Y R \\ 12 \% & 5,000-9,999 \\ 39 \% & 10,000-19,999 \\ 20 \% & 20,000-29,999 \\ 27 \% & 30,000 \text { AND ABOVE }\end{array}$

\section{RESIDENTIAL SETTING}

$\begin{aligned} 54 \% & \text { SUBURBAN } \\ 34 \% & \text { URBAN } \\ 2 \% & \text { RURAL }\end{aligned}$

\section{HOUSEHOLD MARE-UP}

$\begin{array}{ll}74 \% & \text { COUPLE ONLY } \\ 14 \% & \text { CHILDREN AT HOME } \\ 12 \% & \text { OTHER }\end{array}$

MEAN $=34.9 \mathrm{YRS}$.

RANGE $=6-63$

MEAN $=19,440$

MEDIAN $=18,375$ 
households, while $14 \%$ still had children living at home and 12\% were characterized by other living arrangements. A small number of couples (8\%) still had children under 18 at home.

\section{Modes of Transportation}

Prior to the stroke, $83 \%$ of the stroke patients and 76\% of their partners provided their own transportation by private vehicle. Eight percent of the patients and $16 \%$ of the partners depended on their spouse or other relatives for transportation.

\section{ORGANIZATIONAL MEMBERSHIPS AND ACTIVITIES}

\section{Religious Activity}

Religion appeared to be important to this sample of stroke victims and their partners prior to the stroke. of those stroke patients able to participate in the interview, $54 \%$ indicated that religion was somewhat or very important to them. Eighty-four percent of the partners viewed religion as somewhat or very important to them. With regard to religious activity, however, $50 \%$ of the stroke patients were described as inactive and 50\% never attended religious services. About one quarter of stroke patients had been somewhat or very active in their religious group and had attended services at least monthly. Of the partners, $44 \%$ reported that they were someshat or very active, and $46 \%$ attended religious services at least monthly. 


\section{Organizational Membership}

Almost half of the sample ( $46 \%$ of stroke patients and 48\% of partners) reported no organizational memberships or affiliations prior to the stroke. Stroke patients belonged to a total of 73 organizations, mean $=1.46, \mathrm{~S} . \mathrm{D} .=1.91$. Partners named a total of 60 organizations, mean $=1.2, \mathrm{~S} . \mathrm{D}$ = 1.82. Organizations to which stroke victims belonged tended to be fraternal or veterans groups (43\%) or social, sport and recreational groups (31\%). Partners belonged primarily to social, sport and recreational (35\%), fraternal or veterans (24\%), and church-affiliated (20\%) groups.

\section{other Activities}

Subjects were asked about a number of other pre-stroke activities and interests which the literature suggests are associated with subjective well-being in older persons. These included volunteer work, hobbies and interests, and attachment to pets. Information was also obtained about socializing activities inside and outside of the home. Only $16 \%$ of the stroke patients and $24 \%$ of the spouses reported giving time to volunteer work prior to the stroke. Ninety percent of the patients and $94 \%$ of the partners named at least one pre-stroke hobby or interest. Mean number of hobbies was 2.02 for the patients, and 2.3 for the spouses. Fifty-eight percent of the couples had pets, and over $90 \%$ reported being somewhat or very attached to their animals. 
Several questions were asked regarding socializing activities. Responses to these quastions are summarized in Table XV. These couples for the most part were frequent socializers. Depending on the question, only 8-12\% responded that they never socialized prior to the stroke. Two-thirds of respondents had someone over for a meal at least once a month, $59 \%$ went to someone else's home for a meal at least monthly, 61\% had visitors at least weekly, 438 went visiting at least weekly, and $74 \%$ went out with others at least monthly.

HEALTH STATUS

Information concerning the general health status of all of the patients prior to the stroke was obtained. Because of the potential impact of stroke on the health status of the caregiver, a number of questions regarding the pre-stroke health status of the partners were also asked. Health status of stroke patients and partners will be described separately in the fullowing sections.

\section{Health Status of Stroke Patients}

Thirty-eight percent of the patients were considered to be in excellent or very good health prior to the stroke, while $28 \%$ were rated or rated themselves as in poor or fair health. Eighty-two percent had at least one chronic health problem and $46 \%$ had two or more chronic health problems. Thirty-six percent had limiting physical disabilities prior 
TABLE XV

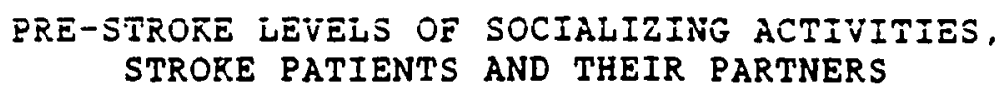

Frequency of Having People Over for a Meal

$\begin{array}{rl}8 \% & \text { NEVER } \\ 25 \% & \text { LESS THAN ONCE A MONTH } \\ 41 \% & 1-3 \text { TIMES A MONTH } \\ 20 \% & \text { WEERLY OR MORE FREQUENTLY } \\ 4 \% & 2-3 \text { TIMES A WEER } \\ 2 \% & \text { DAILY }\end{array}$

Frequency of Going to Someone Else's Home for a Meal

12\% NEVER

29\% LESS THAN ONCE A MONTH

43\% 1-3 TIMES A MONTH

16\% WEEKLY

Erequency of Having Visitors at Home

6\% NEVER

2\% LESS THAN ONCE A MONTH

31\% 1-3 TIMES A MONTH

$27 \%$ WEERLY

$24 \%$ 2-3 TIMES A WEER

$10 \%$ DAILY

Erequency of Going Visiting

$\begin{array}{rl}10 \% & \text { NEVER } \\ 12 \% & \text { LESS THAN ONCE A MONTH } \\ 35 \% & 1-3 \text { TIMES A MONTH } \\ 33 \% & \text { WEERLY } \\ 8 \% & 2-3 \text { TIMES A WEER } \\ 2 \% & \text { DAILY }\end{array}$

Erequency of Going Out with others

$\begin{array}{rl}8 \% & \text { NEVER } \\ 18 \% & \text { LESS THAN ONCE A MONTH } \\ 33 \% & 1-3 \text { TIMES A MONTH } \\ 18 \% & \text { WEEKLY } \\ 21 \% & 2-3 \text { TIMES A WEER } \\ 2 \% & \text { DAILY }\end{array}$


to the stroke. Sixteen patients (32\%) had been hospitalized in the year before the stroke and $12 \%$ had been hospitalized two or more times. These patients were taking a mean of 2.02 pre-stroke medications, S. D. $=1.89$. Eight $(16 \%)$ had experienced appetite changes in the six months before the stroke, and 8 (16\%) had problems sleeping. Only two stroke patients acknowledged a mental health problem for which they wanted or sought help in the six months prior to the stroke. Information about future health expectations was only obtained for those patients who could take part in the interview. At the time of the first interview, 23 (89\%) of the patient respondents reported that they expected their health to get better over the next six months.

\section{Health Status of Partners}

Overall, the sample of partners reported a moderate number of pre-stroke health problems, not an unexpected finding given their age distribution. Forty percent rated their overall health as excellent or very good, while $24 \%$ rated their own health as only fair or poor. Thirty percent reported at least one limiting physical disability. Almost two-thirds (64\%) had at least one chronic health problem, while 26\% reported more than one chronic health problem. Five partners (10\%) had been hospitalized in the year before the stroke. The sample indicated they were taking a mean of 1.5 medications prior to the stroke, S. D. $=1.14$. Few (10\%) reported any appetite changes in the six months prior 
to the stroke, while $14 \%$ mentioned pre-stroke sleep problems. Nine subjects $(18 \%)$ indicated tinat they had experienced a mental health problem in the six months prior to the stroke, and five of nine had sought professional help for this problem. In terms of past health and future health expectations, at the time of first interview $75 \%$ indicated that their health was the same now compared to six months ago, and $75 \%$ expected their health to remain the same over the next six months.

\section{PERCEPTIONS OF THE STRORE}

\section{Patients' Perceptions of the Stroke}

Twenty-six patients (52\%) were able to participate in the interview process at Time 1. Responses of this sample of patients to several questions regarding their perceptions of the stroke are summarized in Table XVI. Regarding the severity of their stroke-related physical problems, $46 \%$ of patients felt that they needed little or no help with daily living activities, $31 \%$ saw themselves as having moderate problems, and 23\% reported serious or very severe problems requiring a lot of help. Forty-two percent reported being somewhat or very concerned that their spouse would not be able to care for them in the future, while $54 \%$ were somewhat or very unconcerned about future care. In response to a question about their level of concern regarding the possibility of another stroke, $31 \%$ were somewhat or very 
TABLE XVI

STRORE PATIENTS' PERCEPTIONS OF THE STROKE, TIME 1

Severity of Stroke-Related Problems

11\% NO PROBLEM

35\% MILD PROBLEM

31\% MODERATE PROBLEM

19\% SEVERE PROBLEM

4\% VERY SEVERE PROBLEM

Concern About the Possibility of Another Stroke

$\begin{aligned} 15 \% & \text { VERY UNCONCERNED } \\ 16 \% & \text { SOMEWHAT UNCONCERNED } \\ 8 \% & \text { NEUTRAL } \\ 38 \% & \text { SOMEWHAT CONCERNED } \\ 23 \% & \text { VERY CONCERNED }\end{aligned}$

Concern About Future Care

29\% VERY UNCONCERNED

25\% SOMEWHAT UNCONCERNED

4\% NEUTRAL

17\% SOMEWHAT CONCERNED

25\% VERY CONCERNED

Anything You Could have Done to Prevent the stroke?

$62 \%$ NO

38\% YES

Attribution of Blame for stroke Occurrence

27\% SELF

15\% CHANCE OR LUCK

8\% HEALTH FACTORS

8\% STRESS

4\% OLD AGE

38\% NO ONE OR DON'T RNOW 
unconcerned while $61 \%$ were somewhat or very concerned. Patients were also asked whether they felt they could have done anything to prevent the stroke and whether they blamed anything or anyone for the stroke happening. Responses to these questions indicated that $62 \%$ did not feel they could have done anything to prevent the stroke. of those who responded affirmatively to this question, three mentioned they could have reduced their stress level, two mentioned adhering to medication regimes, four indicated choices related to diet and other health factors, and one related prevention to paying attention to warning signs. In terms of attributing blame for the stroke, $27 \%$ blamed themselves while almost one-third blamed no one or attributed the stroke to chance or luck. Not surprisingly, spouses, family, and friends were named most frequently as sources of strengh in helping the individual adjust to the stroke. In the early post-stroke period, this sample of patients reported high degrees of satisfaction with health care received for the illness, $77 \%$ stating they were very satisfied.

\section{Partners' Perceptions of the stroke}

The partners' perceptions regarding the stroke at the time of first interview are summarized in Table XVII. It should be kept in mind that Table XVII includes the responses of all 50 partners, including those whose spouses were too seriously impaired to participate in the interview. 
TABLE XVII

CAREGIVERS' PERCEPTIONS OF THE STRORE, TIME 1

Severity of Stroke-Related Problems

$\begin{aligned} 4 \% & \text { NO PROBLEM } \\ 16 \% & \text { MILD PROBLEM } \\ 38 \% & \text { MODERATE PROBLEM } \\ 28 \% & \text { SEVERE PROBLEM } \\ 14 \% & \text { VERY SEVERE PROBLEM }\end{aligned}$

Concern About the Possibility of Another Stroke

12\% VERY UNCONCERNED

6\% SOMEWHAT UNCONCERNED

14\% NEUTRAL

22\% SOMEWHAT CONCERNED

$46 \%$ VERY CONCERNED

Concern About Being Able to Care for the Patient in Future

$26 \%$ VERY UNCONCERNED

8\% SOMEWHAT UNCONCERNED

148 NEUTRAL

24\% SOMEWHAT CONCERNED

28\% VERY CONCERNED

Anything You Could have Done to Prevent the Stroke?

76\% NO Or DON'T RNOW

$24 \%$ YES

Attribution of Blame for stroke Occurrence

$\begin{aligned} 18 \% & \text { STRORE VICTIM } \\ 18 \% & \text { STRESS } \\ 12 \% & \text { HEALTH FACTORS } \\ 8 \% & \text { OTHER PEOPLE } \\ 8 \% & \text { CHANCE/LUCR } \\ 4 \% & \text { SELF } \\ 4 \% & \text { OTHER FACTORS } \\ 28 \% & \text { NO ONE OR DON'T RNOW }\end{aligned}$


Thus, Table XVI and Table XVII are not directly comparable. Forty-two percent of these partners saw the stroke patient as having serious or very severe problems related to physical functioning, and $80 \%$ felt the patient had at least moderate problems. Only $20 \%$ of patients were rated as having minimal or mild problems by their spouses. Feelings of guilt or blame for not having done more to prevent the stroke were expressed by $24 \%$ of the caregivers. These spouses felt that they should have urged the stroke patient to seek medical care or better medical care, and several mentioned monitoring the patient's diet more closely. On the other hand, only two partners specifically "blamed" themselves for the stroke occurrence. Caregivers named family, their religious beliefs, friends, and their own personalities as their greatest sources of strength in coping with the stroke in the early adjustment period. As with the patients, a high proportion of the partners expressed satisfaction with the health care received for the stroke. Eighty percent indicated they were very satisfied with their partner's stroke-related care.

$$
\text { POST-STRORE CHANGES }
$$

\section{Demographics}

Few demographic changes were noted in the six months between interviews. No subjects indicated a change in their marital status. Four couples (8\%) had changed their 
residence. Major changes were noted in the employment status or the stroke victims, as would be predicted. Whereas at Time 118 had been employed full-time, at Time 2 only two persons were employed full-time. The number of stroke patients employed part-time decreased from six to three at Time 2. Employment status of the partners changed minimally from Time 1 to Time 2 . The same number were employed full time at both interview times, and only one person had given up a part-time job. This may suggest the possibility of additional strain on those caregivers who continued to work full-time. On the other hand, most of those employed expressed satisfaction with and enjoyment of their jobs at Time 2. Whereas $83 \%$ of stroke patients provided their own transportation before the stroke, only $22 \%$ were able to do so after the stroke.

Another area of change in demographics was that of finances. Forty percent of caregivers and $38 \%$ of patients indicated that their economic standard of living had decreased either somewhat or considerably since the stroke.

\section{Organizational Memberships and Activities}

Religiosity. At Time 2 there was little change in the relative distribution of responses to the question about the importance of religion in their lives for either the stroke patients or their caregivers. At the same time, religious attendance and perceived degree of religious activity declined. The number of stroke patients who described 
themselves as somewhat or very active in their religious group declined from $24 \%$ to $16 \%$ at Time 2. Frequency of attendance at religious activities remained about the same at Time 2. For the partners, the number who described themselves as somewhat or very active declined from $44 \%$ to 28\%. While $46 \%$ of the caregivers reported that they attended religious services at least monthly before the stroke, only $38 \%$ reported that they attended that frequently at Time 2 .

Other Organizational Activities. For both stroke patients and caregivers total number of organizational memberships claimed decreased at Time 2, while the number who reported no organizational memberships increased. The percentage of stroke victims who named no organizations to which they belonged increased from $46 \%$ at Time 1 to $60 \%$ at Time 2. Total number of organizations named decreased from 73 at Time 1 to 44 at Time 2. For the caregivers, total number of organizations named decreased from 60 at Time 1 to 46 at Time 2, while the percentage of those who claimed no organizational memberships increased from $48 \%$ to $56 \%$.

With regard to hobbies and interests, for the stroke victims, $81 \%$ had at least one hobby or interest they could no longer pursue since the stroke and $45 \%$ had given up two or more hobbies. Only ten of the stroke patients had taken up any new hobbies. Fifty-six percent of the caregivers reported giving up at least one hobby because of the stroke 
and 30\% had given up more than one hobby. Only 7 (18\%) had taken up any new hobbies or interests since the stroke. Social Activities. Somewhat surprisingly, the couples in this sample reported about the same frequency of socializing activities at six months post-onset as they did in describing their pre-stroke socializing patterns. As a group they did not report the decline that might have been expected in the frequency of having people over to their homes, visiting, and going out with other people. Although scores on the Index of Social Activity were slightly reduced at Time 2 for both the caregivers and the patients, these differences were not significant.

\section{Health Status}

Negative changes in self-perceived health and in more objective indicators of health status were observed in the stroke patients and to a lesser degree in the caregivers at Time 2. The number of stroke patients whose overall health was rated as very good or excellent declined from 19 (38\%) at Time 1 to $9(18 \%)$ at Time 2 , while the number whose overall health was rated as fair or poor increased from 14 (28\%) at Time 1 to $21(42 \%)$ at Time 2. About the same number of stroke patients saw themselves or were seen by their partners as having no chronic health problems at Time 2 as at Time 1 ( 9 and 11 respectively). While 23 of the stroke patients were expected to have improved health in six months at Time 1 , only 11 were expected to show improved 
health in six months at Time 2. This, of course, is not surprising, considering that the Time 1 interview occurred quite soon after the onset of the stroke. On the other hand, the fact that fewer were expected to have improved overall health in six months at Time 2 may reflect that the reality of the chronic nature of the stroke disability was acknowledged by more respondents at Time 2 .

Sixteen of the stroke patients (32\%) had been hospitalized again in the six months between interviews, and seven of those had been hospitalized more than once. Eight stroke patients had experienced a mental health problem for which they wanted help since the stroke and five had received some degree of help. Although the actual numbers are small, this represents a four-fold increase in acknowledged mental health problems for the stroke patients compared to pre-stroke levels.

There was also an increase in reported appetite changes and sleep problems for the stroke patients at Time 2. About one-third of the patients were reported to have appetite changes and almost half to have at least one sleep problem at Time 2 . This is twice the number who reported pre-stroke appetite changes and three times the number who reported pre-stroke sleeping problems. In addition to coping with the effects of the stroke, $30 \%$ of the stroke patients experienced at least one additional major negative life event in the six months between interviews. 
As a group the caregivers showed remarkable stability in the numbers and relative proportions of chronic health problems, limiting physical disabilities, and medications. Subjective ratings of present overall health status remained about the same over time as well. On the other hand, the number of caregivers who felt their health overall was worse at the Time 2 interview compared to six months ago increased from $10 \%$ to $26 \%$ and the number who felt that their health would get worse over the next six months increased from $4 \%$ to 8\%. There were some other suggestions that the caregivers were experiencing health stresses as well. The number of acknowledged mental health problems increased from 9 (18\%) at Time 1 to $16(32 \%)$ at Time 2 for the caregivers. Eleven of the subjects had sought professional help for these problems and 1 had sought informal help, while one subject had sought both. While 45 subjects (90\%) had reported no recent appetite changes at Time 1 , this number declined to $34(68 \%)$ at Time 2. Likewise, while 43 (86\%) had reported no recent sleep disturbances at Time 1 , this number had declined to 33 (66\%) at Time 2. Finally, 468 of the caregivers reported experiencing at least one major negative life event in the six months between interviews.

CONCLUSION

In summary, there were few demographic changes over time for this group of stroke patients and their partners 
with the exception of stroke patient employment status. Decreased participation in religious activities was seen over time for the caregivers, and both groups showed a decline in organizational memberships and hobbies. The strain expected to occur in those primarily caring for chronic stroke patients was seen in an increasing number of subjective mental health problems and some decrease in optimism about their own physical health. Stroke patients showed a number of subjective health changes as well.

The next chapter will continue the descriptive analysis of the data as the social networks and social support systems of the subjects are examined. 


\section{CHAPTER V}

DESCRIPTIVE ANALYSIS: SOCIAL NETWORRS AND SOCIAL SUPPORT

Because the major purpose of this study was to investigate the relationships between social network and social support characteristics and well-being outcomes in this sample of stroke patients and their partners, this chapter will describe several aspects of the social environments of respondents. Network characteristics and support functions were analyzed on two levels of closeness, or intimacy. First, specific characteristics of the larger social networks of the sample will be briefly discussed.

\section{SUBJECTS' OVERALL SOCIAL NETWORRS}

Names of network members, up to a total of 20 , were elicited by asking subjects to first identify those persons who provided, or for whom they provided specific types of instrumental, emotional, and informational support or with whom they socialized on a regular basis. They were also asked to provide the names of any other persons who were important to them, or with whom they regularly interacted. Information about the gender and relationship status of these individuals to the respondent was also obtained.

At the time of the first interview, subjects described their pre-stroke social networks. Caregivers were then asked detailed questions about the people who had provided 
specific kinds of assistance during the early post-stroke period. The stroke pacients who could participate in the interview process were asked the same questions to elicit the names of persons in their network before the stroke, and one question concerning "who has been important and helpful" during the early post-stroke period. For those patients who could not respond for themselves, partners were asked a shortened series of questions to elicit the names of the important people in the stroke victim's pre-stroke social network and a single question regarding the network during the early post-stroke period. At Time 2, the frame of reference for eliciting the names of network members was "over the past few months".

Thus, for the caregivers, the following discussion will concern social networks at three points in time: before the stroke; during the early post-stroke period; and at the time of the second interview. For the stroke victims, analysis of network characteristics will involve just two points in time: the pre-stroke network and the network as perceived at the Time 2 interview. Because very limited information was obtained about the social networks of the stroke patients during the immediate post-stroke period, these data will not be included in further analyses.

\section{Careqivers' Overall Social Networks}

Pre-stroke Social Networks. Table XVIII summarizes characteristics of the partners' pre-stroke social networks 
TABLE XVIII

CAREGIVERS'OVERALL PRE-STROKE SOCIAL NETWORRS

Total Number of Network Members

Mean Number of Network Members

Range

Gender Composition

Female

Male

Relationship Composition

$\begin{array}{lccc} & \text { Total \# } & \text { 文\# } & \text { \& NW } \\ \text { Female } & 357 & 7.14 & 59 \\ \text { Male } & 253 & 5.06 & 41\end{array}$

610

12.20, S. D. $=4.30$

$4-20$

\begin{tabular}{lccc} 
& Total \# & $\overline{\mathrm{x} \#}$ & $\underline{\text { \% NW }}$ \\
\cline { 2 - 3 } & 213 & 4.26 & 35 \\
Children & 103 & 2.05 & 17 \\
Other Relatives & 99 & 1.98 & 16 \\
Neighbors & 78 & 1.56 & 13 \\
Spouse & 43 & .86 & 7 \\
Siblings & 29 & .58 & 5 \\
Work Associates & 19 & .38 & 3 \\
Parents & 14 & .28 & 2 \\
Other & 12 & .24 & 2 \\
& & & 47 \\
Total Relatives & 288 & 5.76 & 53 \\
Total Non-Relatives & 322 & 6.44 &
\end{tabular}


including size, gender composition and relationship composition. This sample of caregivers named a total of 610 ties, for a mean network size of 12.20, S. D. = 4.3. Networks ranged in size from to 20 persons. They were predominantly composed of females (59\%) and friends (35\%). Caregivers named more non-kin (53\%) than kin in their prestroke social networks.

With regard to network size, the networks of this sample of primarily older, female caregivers are comparable to those of other network studies (Stueve Fischer, 1978; Wellman, 1985) in which the network eliciting questions were asked in a similar way, and with data which indicates that, depending on how the question is asked, most people name between 8 and 15 persons in their personal networks (Schulz \& Rau, 1985). Given the age distribution of the sample, together with evidence in the literature which indicates that same-sex friends and women are most frequently cited as sources of social support (Griffith, 1985), it is not a surprising finding that the caregivers in this sample included more females than males in their supportive networks .

With regard to relationship composition of the network, the relative number and proportion of kin, friends, neighbors, and work associates in this sample were similar to those of the older respondents in the Northern California Communities Study (Fischer, 1982; Stueve \& Fisher, 1978). 
Respondents named a nean number of $\$ .26$ friends, 1.56 naighbora, and 5.75 raletives in their pre-stroke networks. Forty-four caregivers (88\%) named the stroke patient as part of their pre-stroke network.

Barly Post-Stroke Networks. Caregiver respondents were asked a series of specific questions about the people who had provided various types of support in the period of initial adjustment to the stroke occurrence. Table XIX summarizes the size, gender, and relationship composition information for the overall social networks at this time period. Size and gender composition of the networks are essentially unchanged from pre-stroke levels. Friends, other relatives, and neighbors appear to assume slightly more importance during this period of support mobilization. Several subjects mentioned specifically the many helpful things that neighbors had done for them while they were spending more time at the hospital and traveling back and forth. The number of children reported as being helpful during the stroke "crisis" period declined for the apparent reason that a number lived too far away to provide specific, tangible help although they remained important members of the network. The proportion of non-relatives who provided support during the stroke crisis period increased from $53 \%$ to $61 \%$ compared to the pre-stroke period. Overall, these results appear to reflect an expected "rallying around" phenomenon on the part of the support system, 
TABLE XIX

CAREGIVERS' OVERALL SOCIAL NETWORRS,

EARLY POST-STRORE PERIOD

Total Number of Network Members

Mean Number of Network Members

Range

Gender Composition

Female

Male

Relationship Composition

\begin{tabular}{lccc} 
& Total $\#$ & $\overline{\mathrm{x}} \#$ & $\underline{8 \mathrm{NW}}$ \\
\cline { 2 - 3 } & 220 & 4.40 & 38 \\
Friends & 107 & 2.14 & 18 \\
Other Relatives & 97 & 1.94 & 17 \\
Neighbors & 78 & 1.56 & 13 \\
Children & 32 & .64 & 6 \\
Siblings & 20 & .40 & 3 \\
Work Associates & 18 & .36 & 3 \\
Other & 8 & .16 & 1 \\
Parents & 5 & .10 & 1 \\
Spouse & & & 61
\end{tabular}

$\begin{array}{ccc}\text { Total \# } & \text { X } \# & \text { \&NW } \\ 340 & 6.80 & 58 \\ 245 & 4.90 & 42\end{array}$

585

$11.70, S . D .=4.56$

$4-20$ 
despite the fact that the overall networks are, in fact, slightly smaller during the early post-stroke period. Network eliciting questions pertaining to the stroke crisis period dealt only with specific types of stroke-related assistance provided, and would not have been expected to elicit as many names as the more general support questions asked about the pre-stroke network.

Time 2 Networks. Overall characteristics of the caregivers' networks at Time 2 are found in Table $\mathrm{XX}$. Unexpectedly, the partners reported a greater number of network members at Time 2 (total $=707 ; \bar{x}=14.14, \mathrm{~S} . \mathrm{D} .=$ 4.40; range $=6-20$ ) than in their pre-stroke networks. The proportion of females named as members of the network increased slightly over time, from $58 \%$ to $62 \%$. At six months after the initial interview, little change was seen in the relationship composition of the active networks of these caregivers. Figure 2, which compares network relationship composition for the major categories of relationsip at the three points in time, illustrates the degree of stability in the make-up of the networks. The relative proportions of friends and neighbors had returned to pre-stroke levels by Time 2. The proportion of kin versus non-kin in the network remained exactly the same over time.

\section{Respondent Stroke Patients' Overall Social Networks}

Pre-Stroke Networks. Table XXI summarizes the size, 
TABLE XX

CAREGIVERS' OVERALL SOCIAL NETHORRS, TIME 2

Total Number of Network Members

Mean Number of Network Members

Range

Gender Composition

Female

Male

Relationship Composition

Total \#

Friends

Other Relatives

Children

Neighbors

Siblings

Spouse

Other

Parents

Work Associates

Total Relatives

Total Non-Relatives

Total \# 437

270

248

139

104

94

42

35

25

11

9

331

376
707

$$
\begin{aligned}
& 14.14, S . D .=4.40 \\
& 6-20
\end{aligned}
$$

\&NW

62

38
$4.96 \quad 35$

$2.78 \quad 20$

$2.08 \quad 15$

1.88

13

6

5

3

2

1

47

53 


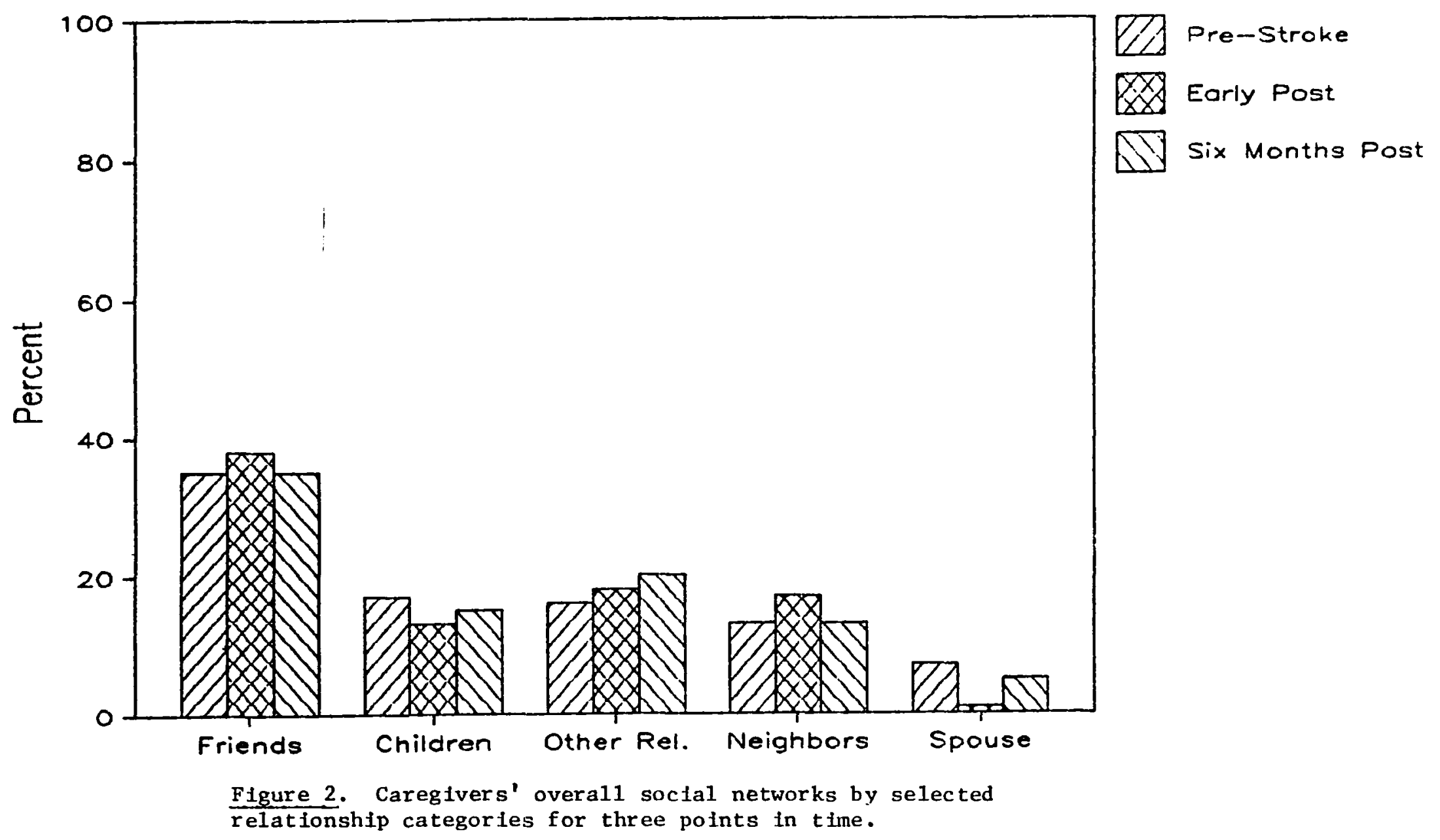


TABLE XXI

STRORE DATIENTS' PRE-STRORE OVERALL SOCIAL NETWORRS (INCLUDES ONLY THOSE ABLE TO BE INTERVIEWED, $N=25$ )

Total Number of Network Members

Mean Number of Network Members

Range

Gender Composition

\section{Female \\ Male}

\section{Relationship Composition}

\begin{tabular}{|c|c|c|c|}
\hline & Total \# & $\underline{\bar{x}} \#$ & \% NW \\
\hline Friends & 100 & 4.00 & 35 \\
\hline Other Relatives & 64 & 2.56 & 22 \\
\hline Children & 47 & 1.88 & 16 \\
\hline Neighbors & 33 & 1.32 & 12 \\
\hline Spouse & 22 & .88 & 8 \\
\hline Siblings & 11 & .44 & 4 \\
\hline Work Associates & 7 & .28 & 2 \\
\hline Parents & 3 & .12 & 1 \\
\hline other & 1 & .04 & 0 \\
\hline Total Relatives & 147 & 5.88 & 51 \\
\hline Total & 141 & 5.64 & 49 \\
\hline
\end{tabular}

\section{8}

$11.52, \mathrm{~S} . \mathrm{D} .=4.69$

$1-18$

$\begin{array}{ccc}\text { Total \# } & \underline{\bar{x} \#} & \text { 㝵W } \\ 144 & 5.76 & 50 \\ 144 & 5.76 & 50\end{array}$


gender composition, and relationship composition information for the pre-stroke social networks of the stroke victim respondents. Detailed social network information was obtained from 25 patients who participated in the interview. They reported a total of 288 network ties $(\bar{X}=11.52, S . D .=$ 4.69, range $=1-18)$. Their networks were composed of an equal number of males and females. Relationship composition showed some differences compared to the partners' networks. Other relatives appeared to be more important in the support systems of the patients before the stroke. Likewise, they named a slightly greater proportion of relatives in their networks than non-relatives. Overall, these data on the pre-stroke networks of the patients are in agreement with other comparable studies (Fischer, 1982; Stueve \& Fischer, 1978; Wellman, 1985). Eighty-eight percent of the patients also named their partners as part of their network.

Time 2 Networks. At Time 2, detailed network information was obtained from 24 patients. These data are summarized in Table XXII. Again contrary to expectations, size of the network did not decline at Time 2 for this subgroup of patients. In fact, size of the network increased at Time 2 . Whereas at Time 1 patients had named a total of 288 network members, at Time 2 they reported a total of 316 ties $(\bar{X}=13.17 ;$ S. D. $=4.46)$. Number of network persons named ranged from 6 to 20 . In examining the gender composition data, it appears that virtually all of 
TABLE XXII

STRORE PATIENTS' OVERALL SOCIAL NETHORRS, TIME 2 (INCLUDES ONLY THOSE ABLE TO BE INTERVIEWED, $\mathrm{N}=24$ )

Total Number of Network Members

Mean Number of Networ's Members

Range

Gender Composition

Female

Male

$\begin{array}{lccc} & \text { Total \# } & \bar{x} \# & \text { \% NW } \\ \text { Friends } & 91 & 3.79 & 29 \\ \text { Other Relatives } & 80 & 3.33 & 25 \\ \text { Children } & 54 & 2.25 & 17 \\ \text { Neighbors } & 37 & 1.54 & 12 \\ \text { Spouse } & 23 & .96 & 7 \\ \text { Siblings } & 14 & .58 & 5 \\ \text { Other } & 9 & .38 & 3 \\ \text { Parents } & 4 & .17 & 1 \\ \text { Work Associates } & 4 & .17 & 1 \\ & & & 4.29 \\ \text { Total Relatives } & 175 & 5.88 & 45 \\ \text { Total Non-Relatives } & 141 & & \end{array}$

316

13.17, S. D. $=4.46$

$6-20$
Total \#

171

145

6.04
8 NW 54 46

\section{Relationship Composition}


the network members added to the increased total of 316 were İemales.

Examination of relationship composition data reveals some interesting findings. The number and proportion of friends in the stroke victims' networks declined at Time 2 , while the number and proportion of relatives named increased. Other relationship categories maintained their relative proportions at Time 2 . Comparison of Time 1 and Time 2 data for the most important relationship categories is illustrated in Figure 3. Twenty-three of 24 patients included their partners as members of their networks at Time 2 .

Non-Respondent Stroke Patients' Overall Social Networks

As previously indicated, a limited number of questions were employed to obtain information about the pre-and poststroke social networks of those patients who could not respond for themselves. These data were provided by the caregiver and are summarized in Tables XXIII and XXIV.

Pre-Stroke Networks. At Time 1, caregivers were asked to indicate those persons with whom the stroke patient regularly spent time socializing, in whom she or he would confide, and any other people important to the patient before the stroke occurrence. Detailed questions about instrumental assistance or reciprocal support were not asked. Table XXIII indicates the size, gender, and relationship composition characteristics of these 24 stroke 

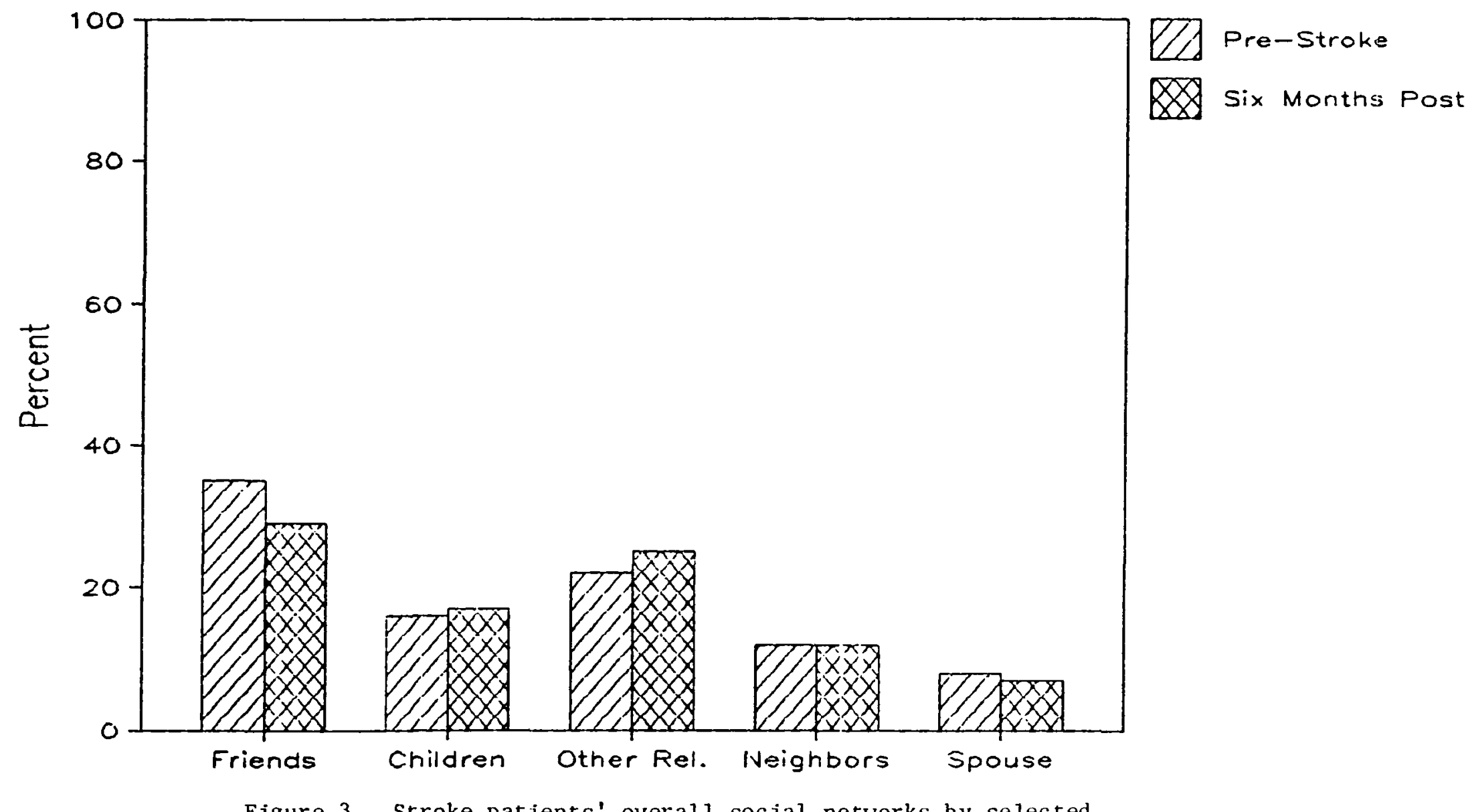

Figure 3. Stroke patients' overall social networks by selected relationship categories for two points in time. 
TABLE XXIII

NON-RESPONDENT STRORE PATIENTS' PRE-STRORE OVERALL SOCIAL NETWORKS $(N=24)$

Total Number of Network Members

Mean Number of Network Members

Range
206

$8.58, S . D=3.61$

$1-15$

\section{Gender Composition}

$\begin{array}{lccc} & \text { Total \# } & \underline{\bar{x} \#} & \underline{\text { NW }} \\ \text { Female } & 90 & 3.75 & 44 \\ \text { Male } & 116 & 4.83 & 56\end{array}$

\section{Relationship Composition}

\begin{tabular}{lccc} 
& Total & $\bar{x} \#$ & \% NW \\
\cline { 2 - 3 } & 92 & 3.83 & 45 \\
Children & 37 & 1.54 & 18 \\
Spouse & 21 & .88 & 10 \\
Other Relatives & 16 & .67 & 8 \\
Work Associates & 15 & .63 & 7 \\
Siblings & 11 & .46 & 5 \\
Neighbors & 9 & .38 & 4 \\
Other & 3 & .13 & 2 \\
Parents & 2 & .08 & 1 \\
& & & 42 \\
Total Relatives & 87 & 3.62 & 58 \\
Total Non-Relatives & 119 & 4.96 &
\end{tabular}


TABLE XXIV

NON-RESPONDENT STRORE PATIENTS' TIME 2

OVERALL SOCIAL NETWORRS $(N=25)$

Total Number of Network Members

Mean Number of Network Members

Range

$$
\begin{aligned}
& 9.00, S . D=4.43 \\
& 2-20
\end{aligned}
$$

\section{Gender Composition}

$\begin{array}{ccc}\text { Total \# } & \text { X \# } & \text { \&NW } \\ 116 & 4.64 & 52 \\ 109 & 4.36 & 48\end{array}$

\section{Relationship Composition}

\begin{tabular}{lccc} 
& Total \# & $\bar{X} \#$ & N N \\
\cline { 2 - 3 } & 71 & 2.84 & 31 \\
Children & 36 & 1.44 & 16 \\
Other Relatives & 36 & 1.44 & 16 \\
Spouse & 24 & .96 & 11 \\
Neighbors & 24 & .96 & 11 \\
Other & 16 & .64 & 7 \\
Siblings & 11 & .44 & 5 \\
Parents & 4 & .16 & 2 \\
Work Associates & 3 & .12 & 1 \\
& & 4.44 & 49 \\
Total Relatives & 111 & 4.56 & 51 \\
Total Non-Relatives & 114 & &
\end{tabular}


patients' networks. Caregivers who were reporting on the pre-stroke social networks of their partners indicated a total of 206 network ties, with a $\bar{x}$ of 8.58 and a range of 1 to 15. Gender composition of the networks was $56 \%$ male. Forty-five percent of the ties were friend relationships, and 58\% of network members were non-relatives. While there are size, gender, and relationship composition differences in these networks compared to the pre-stroke networks of those patients who reported for themselves, there is no reason to suspect that the networks of the two groups of patients would have necessarily been different before the stroke. Two factors, at least, were operating to cause these apparent differences. First, partners would not necessarily perceive or report network membership in the same way as would persons reporting for themselves. Second. the questions asked to elicit network names were different for the two groups of patients, such that certain categories of network members (for example, neighbors) would be much less likely to appear on the network lists of those patients not able to describe their own networks.

Time 2 Networks. Table XXIV summarizes the social network characteristics of the non-respondent stroke patients at Time 2. As was true for the other groups of subjects, no decline in network size was found at Time 2 . Gender composition changed from 56\% male at Time 1 to $52 \%$ female at Time II. A similar change in network gender 
composition was observed for the stroke patients who reported for themselves. The number and proportion of friends in the network declined at Time 2 , as did the number of work associates. Neighbors and other relatives appeared to become more important network members. The number of non-relatives in the network at Time 2 declined from $58 \%$ to 51\%. Figure 4 summarizes the relative proportions of several relationship categories for the pre- and post-stroke networks of the non-respondent stroke patients.

\section{Distribution of Change in Size of Overall Networks}

Although examination of the group data suggested that respondents did not experience decreases in the size of their overall social networks over time, it was suspected that certain individuals within the caregiver group and the two patient groups may have had significant declines in the size of their networks following the stroke. For this reason, the distribution of change in the size of overall networks was determined. In the data reported below, a negative value indicates an increase in the size of the network at Time 2 .

Partners experienced a mean change score of -1.86 ( $\mathrm{S}$. D. $=4.74$, range $=-12$ to 10$)$. Twenty-seven of the caregivers had an increase in network size, nine reported no change, and 14 had smaller overall networks at Time 2 . For 11 subjects, increase in size of the network was more than one S. D. from the mean, and for 3 persons, decrease in 


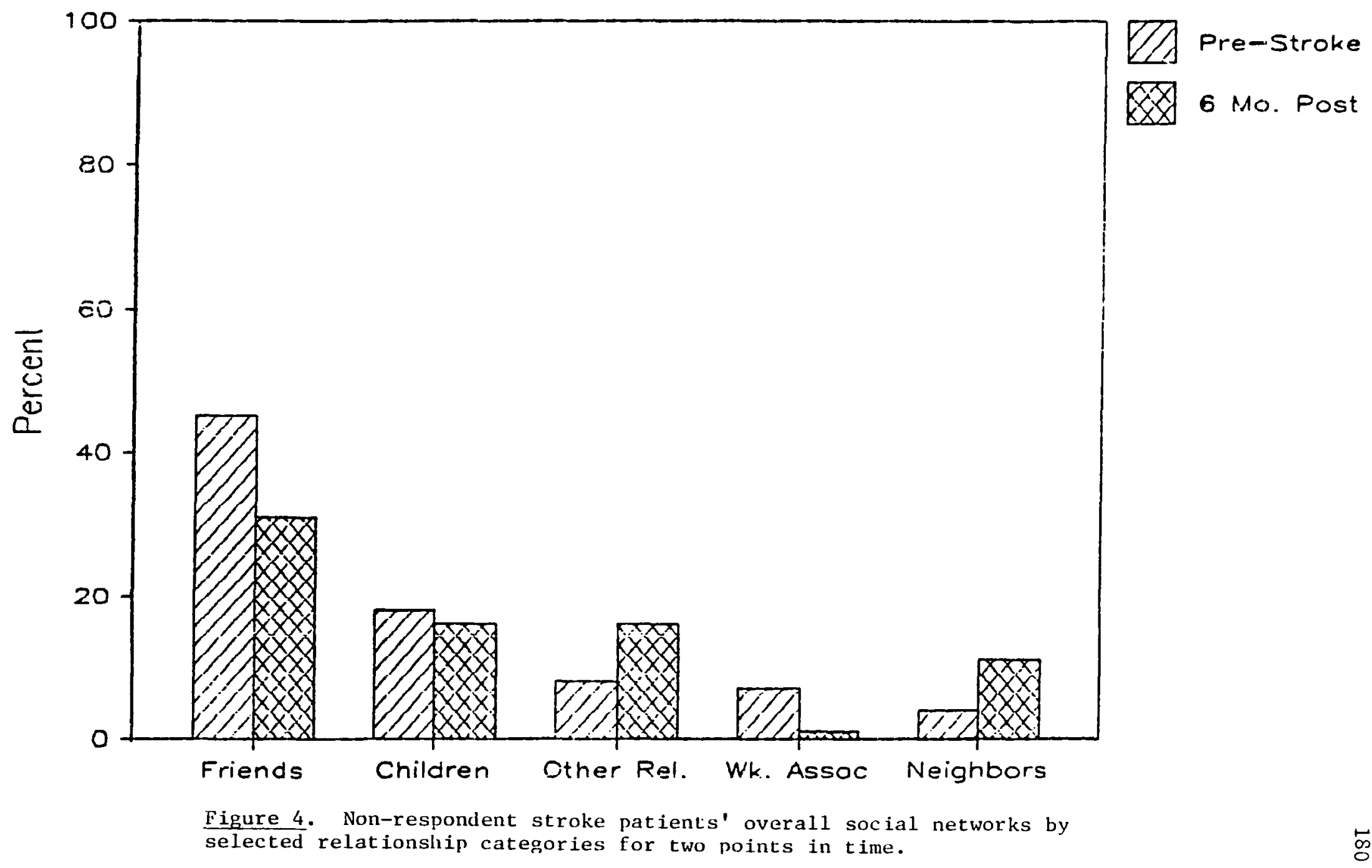


network size was more than one $S$. D. from the mean.

Stroke patients who responded For themselves had similar results to those found for the caregivers. They experienced a mean increase in size of the overall network $(\bar{x}=-1.667$, S. D. $=4.35$, range $=-11$ to 8$)$. Two-thirds $(16 / 24)$ reported larger networks at Time 2 , while about onethird (7/24) reported smaller networks at Time 2. Five patients had an increase in network size greater than one $\mathrm{S}$. D. from the mean, while only two had a decrease in network size which was more than one $S$. D. from the mean.

For the non-respondent stroke patients, a somewhat different picture emerged when change data were analyzed. This subgroup of patients experienced a mean decrease in network size over time $(\bar{X}=1.32$, S. D. $=5.82$, range $=-13$ to 11). Twice as many of these patients (16/25) experienced a decrease in network size as had an increase in the size of the network at Time 2. Four had an increase in network size greater than one S. D. from the mean, while 6 (about onefourth) had a decrease in the size of the overall network which was more than one $S$. D. from the mean.

\section{Summary: Overall Social Networks}

Contrary to expectations, neither the stroke patients nor their partners reported declines in the size of their overall social networks as a result of the stroke when group data were considered. In fact, the caregivers reported a significant increase in the size of the overall network over 
time. On the other hand, examination of the distribution of change data indicated that a few caregivers and respondent stroke patients did experience substantial declines in the size of their overall networks over time, while two-thirds of the non-respondent patients had smaller networks at Time 2. About one-fourth of these patients reported significant declines in the size of their overall networks after the stroke.

Except for an increase in support from non-relatives during the early post-stroke period, caregivers' networks showed stability over time in terms of relationship composition, and a slight increase in the proportion of females in the network (from 598 to 628).

On the other hand, changes in both gender composition and relationship composition were observed within the patients' networks. These changes are summarized in Table XXV. If the stroke patients' networks are considered together, gender composition changed over time from predominantly male (53\%) at Time 1 to predominantly female 1538) at Time 2. This appeared to be the result of the addition of female network members at Time 2, rather than the result of any appreciable decline in male network members. Friends and work associates named as network members declined in both number and proportion over time, and other relatives and neighbors increased in number and as a percent of the total network. The proportion of relatives 
TABLE XXV

\section{CHANGESS IN THE OVERALL SOCIAL NETWORRS OF STRORE PATIENTS FROM TIME 1 TO TIME 2}

TIME 1

TOTAL \#\# $\underline{\text { \# }}$

234

260

Male
Gender Composition

Female

Relatioriship Composition *

Friends

Other Relatives

Neighbors

Work Assoc.

other

Total Relatives

Total Non-Rel.
192

80

42

22

4

234

260
39

16

9

4

1

47

53

TIME 2

TOTAL \#\#

287

53

254

47

53

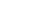

$\$$ 
to non-relatives increased from $47 \%$ at Time 1 to 538 at Time 2. Similar to the findings regarding gender composition, this shift was the result of kin being added to the network at Time 2 rather than the result of significant decline in the number of non-kin named. The importance of these observed changes in overall network composition for the patients will be explored in Chapter VI.

\section{SUBJECTS INTIMATE SOCIAL NETWORRS}

After network lists had been completed, subjects were asked to indicate which of the network members (up to a total of ten) they felt closest to, or were the most important to them. These network members became part of the individual's intimate, or close network. For those subjects who named ten or fewer persons in their overall networks, all were included as members of the intimate network. A few subjects who had named more than ten persons in their overall networks refused to choose ten for their close network. In these cases, the number of network members chosen determined the size of the intimate network.

Additional information was obtained about the close networks, including age of the network member, duration of the relationship, frequency of contact, geographical distance from the focal individual, who usually initiated the contact (symmetry), and which of the other network members they knew (density). All further analyses of the 
network data, including the types and amount of social support provided and the degree of reciprocity in the relationship were completed for the intimate networks only. The following sections of the chapter describe the intimate social networks of the subjects. The data for the stroke patients who could participate in the interview and for those who could not are included together.

\section{Caregivers' Intimate Social Networks}

Data sumarizing the characteristics of the caregivers' intimate social networks for Time 1 and Time 2 are found in Tables XXVI, XXVII, and XXVIII. Partners named a mean number of 8.82 persons in their close networks at Time 1, with essentially no change at Time 2 . They had known their network members for a long time, a mean of 28.09 years. Average age of network members was 49 years. There was a slight, but not significant drop in average length of time network members had been known at Time 2 , and no change in the average age of close network ties. These close networks of the caregivers contained a higher proportion of females (62\% and $64 \%$ at Time 1 and Time 2 respectively) than did their larger networks. In sum, there was little change in the gender, age, size, and duration of relationship characteristics of the caregivers' intimate networks over time. This parallels the stability found in their larger their larger networks. We had anticipated at least finding changes in network size, hypothesizing that networks would 
TABLE XXVI

CḦARACTERISTICS OF SUBJECTS'IATIMATE SOCIAI NETWORRS

TIME 1

$\underline{\bar{x}} \quad \underline{S . D}$.
TIME 2

$\overline{\mathrm{X}} \quad$ S.D.

\section{Stroke Patients}

$\begin{array}{lrrrr}\text { Number in NW } & 8.08 & 2.44 & 7.79 & 2.59 \\ \begin{array}{l}\text { Number of Years } \\ \text { Rnown }\end{array} & 29.18 & 11.05 & 25.76 & 11.78 \\ \text { Age of NW Members } & 50.46 & 11.80 & 49.19 & 12.00\end{array}$

Caregivers

$\begin{array}{lrrrr}\text { Number in NW } & 8.82 & 1.59 & 8.96 & 1.54 \\ \begin{array}{l}\text { Number of Years } \\ \text { Rnown }\end{array} & 28.09 & 9.92 & 26.53 & 9.91 \\ \text { Age of NW Members } & 48.93 & 10.77 & 48.96 & 9.02\end{array}$

* Number of valid cases for S.V. at Time $2=49$ 
TABLE XXVII

GENDER COMPOSITION OF SUBJECTS'INTIMATE SOCIAL NETWORKS

TIME 1

TIME 2

TOTAL

$\underline{8}$

TOTAL

$\underline{\text { z }}$

Stroke Patients

$\begin{array}{lllll}\text { Female } & 202 & 50 & 201 & 53 \\ \text { Male } & 203 & 50 & 181 & 47\end{array}$

\section{Caregivers}

$\begin{array}{lllll}\text { Female } & 277 & 62 & 285 & 64 \\ \text { Male } & 167 & 38 & 163 & 36\end{array}$

* Number of valid cases for $S$. V. at Time $2=49$ 
TABLE XXVIII

CAREGIVERS' INTIMATE SOCIAL NETWORR COMPOSITION BY TYPE OF RELATIONSHIP

TIME 1

RELATIONSHIP
CATEGORY
Friends
Children
Other Relatives
Spouse
Neighbors
Siblings
Work Friends
Other
Parents

Totals
TOTAL \#

120

103

91

41

32

31

9

9

8

444

TIME 2

TOTAL

124

28

97

22

93

21

33

7

42

38

(2)

9

1

1

9

2

$446 \quad 100$ 
become smaller over time as a result of the stroke. These results suggest, however, that at least during the first six to eight months following a stroke, the intimate and effective network zones (Boissevain, 1974) do not shrink for the group represented by this sample.

Likewise, few changes were found over time in the relationship composition of the partners' close networks (Table XXVIII and Figure 5). Close friendships are maintained at least to the degree that the number of friends mentioned as part of the close network does not decline. There is a hint in the data that neighbors and siblings may become more important in a chronic illness situation, in that they are included in the intimate network more frequently at Time 2. Fewer caregivers mentioned their partners as members of their close networks at Time 2 (41 at Time 1 versus 33 at Time 2 ).

\section{Stroke Patients' Intimate Social Networks}

Tables XXVI, XXVII, and XXIX and Figure 6 summarize the information about the patients' close networks. As was the case for the caregivers, there was little change in average network size or in mean age of network members over time. The average number of years network members had been known declined from 29.18 years at Time 1 to 25.76 at Time 2. This would suggest that, for some patients at least, new people moved into the closest network sector. The gender composition of the patients intimate networks changed in 


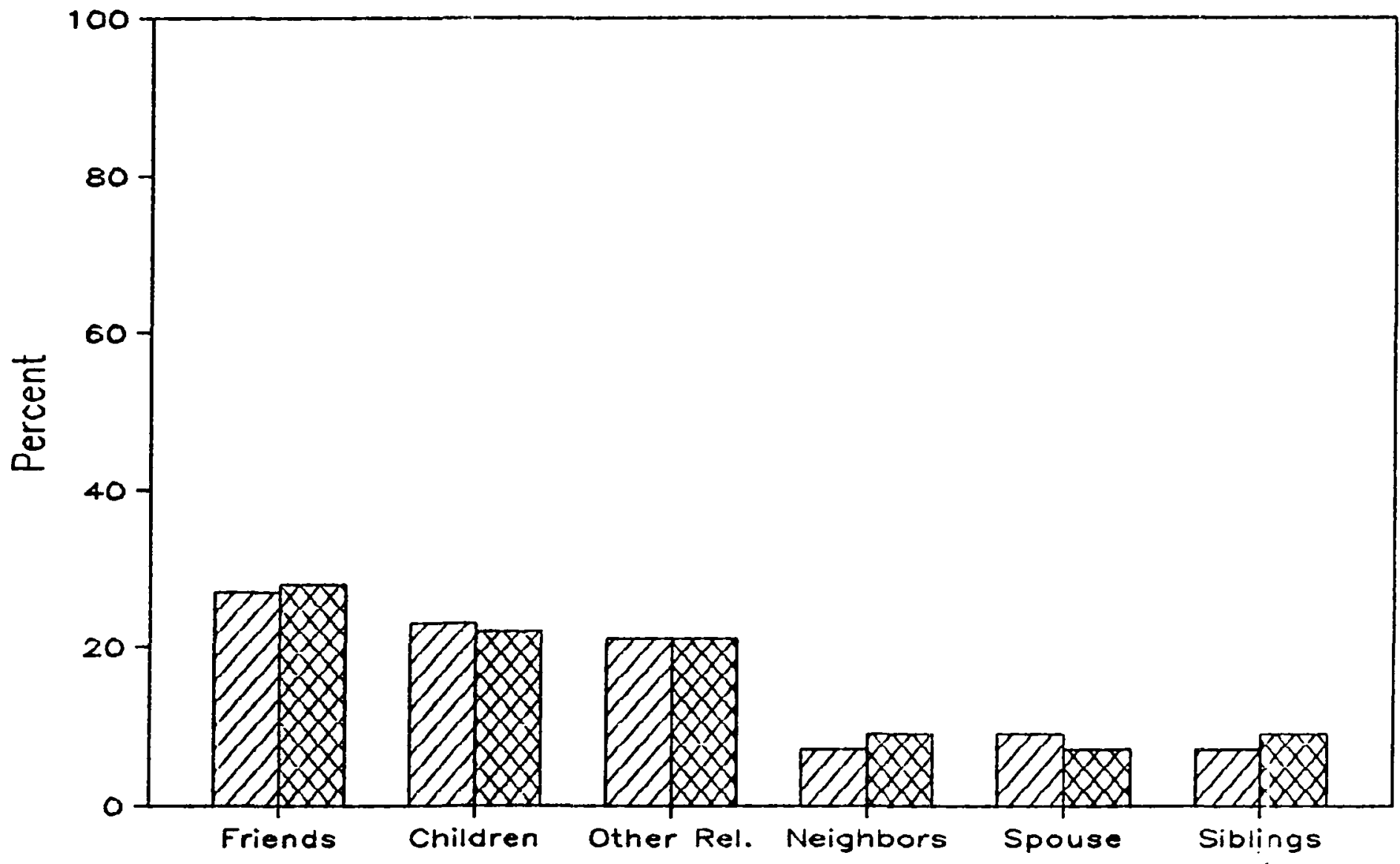

Q 7ї: $=1$
rime 2

Figure 5. Caregivers' intimate social networks by selected relationship categorfes for two points in time. 
TABLE XXIX

STRORE FATIENTS' INTIMATE SOCIAL NETHORK COMPOSITION

BY TYPE OF RELATIONSHIP

\begin{tabular}{|c|c|c|c|c|c|}
\hline & \multicolumn{2}{|c|}{ TIME 1} & \multicolumn{3}{|c|}{$\underline{\operatorname{TIME} 2}{ }^{*}$} \\
\hline $\begin{array}{l}\text { RELATIONSHIP } \\
\text { CATEGORY }\end{array}$ & TOTAL \# & $\underline{z}$ & & TOTAL \# & 9 \\
\hline Friends & 124 & 31 & & 96 & 25 \\
\hline Children & 88 & 22 & & 81 & 21 \\
\hline Other Relatives & 76 & 19 & & 85 & 22 \\
\hline Spouse & 44 & 11 & & 45 & 12 \\
\hline Siblings & 24 & 6 & & 21 & 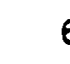 \\
\hline Neighbors & 19 & 5 & & 34 & 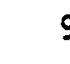 \\
\hline Work Friends & 18 & 4 & & 4 & \\
\hline Parents & 6 & 1 & & 8 & \\
\hline Other & 3 & 1 & & 8 & \\
\hline Totals & 402 & & 382 & 10 & \\
\hline
\end{tabular}

* Number of valid cases for S.V. at Time $2=49$ 


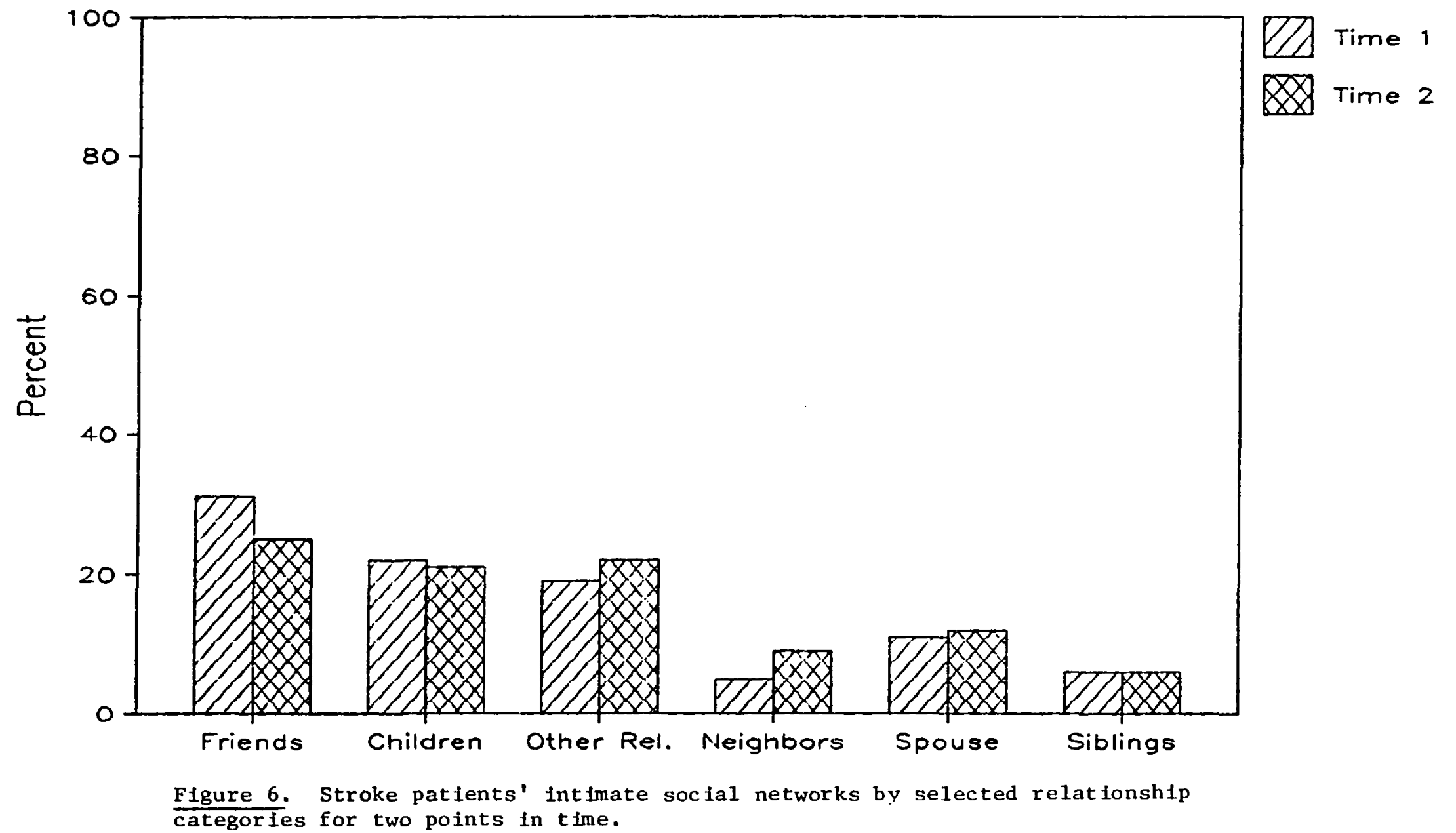


that the number of female network members remained constant, while the number of males declined. This may reflect in part the loss of work associates. On the other hand, few work associates were included in the close networks of the patients at Time 1.

Erequency of Network Contact

Frequency of contact with non-household network members was determined for face-to-face contacts, telephone and other types of contacts, and for combined contacts (a summed total of face-to-face and other interactions). Rather than ask respondents to recall the actual number of contacts with individual network members during a specified time period (e.g., the past month), a typical or "on the average" frequency of contact scale was utilized which ranged from $0=$ "never" to $8=$ "daily". Framing the frequency of contact question in this way allowed respondents to report more typical or representative information regarding frequency of social network interaction. Frequency of contact data are summarized in Tables XXX and XXXI.

Time 1 Frequency of Contact. At Time 1 , caregivers reported a total number of 398 members of the close network with whom they had face-to-face contact $(\bar{X}=7.96$, S. D. $=$ 1.70, range $=2-101$ and 387 with whom they had telephone and other types of contacts $(\bar{X}=7.74$, S. D. $=1.82$, range $=$ 2-10). These were not, however, mutually exclusive groups, 
TABLE XXX

\section{CAREGIVERS' FREQUENCY OF NETWORR CONTACTS, TIME 1 AND TIME 2}

$$
\frac{\text { Time } 1}{(N=50)} \quad \frac{\text { Time } 2}{(N=49)}
$$

Total \# of NW Members, Face-to-Face Contact

Average \# of NW Members, Face-to-Face Contact

Total \# of NW Members, Other Contacts

Average \# of NW Members, other Contacts

Average Frequency of NW Contacts:

Face-to-Face Contacts:

ONCE A MONTH OR LESS

2-3 TIMES A MONTH WEERLY

GREATER THAN WEERLY

Other Contacts:

ONCE A MONTH OR LESS 2-3 TIMES A MONTH WEERLY

GREATER THAN WEERLY

$\begin{array}{rlrr}15 & (30 \%) & 13 & (27 \%) \\ 18(368) & 18 & (37 \%) \\ 12(24 \%) & 13 & (27 \%) \\ 5(10 \%) & 5 & (10 \%)\end{array}$

$10 \quad(20 \%)$

$6(12 \%)$

$13(27 \%)$

$14(298)$

$13(26 \%)$

$10(20 \%)$

\section{All Contacts:}

ONCE A MONTH OR LESS 2-3 TIMES A MONTH WEERLY

GREATER THAN WEEKLY 
in that many network members engaged in both face-to-face and other contacts with respondents. Partners reported seeing their network members two to three times a month, on the average. They had other types of contacts with network members almost weekly, on the average. For all types of contacts, caregivers reported an average frequency of slightly more often than once a week. Nine respondents (18\%) reported average contact frequency (of all types) to be once a month or less, and these individuals might be viewed as having low frequency of contact with members of their networks. Five caregivers (10\%) reported high frequency of contact with network members, having contact several times a week, on the average.

Table XXXI summarizes the frequency of network contact data for the stroke patients. For the patients at Time 1 , there were 345 network members with whom they had face-toface contact $(\bar{x}=6.90, S . D .=2.59$, Range $=0-10)$. A total of 321 network members had other types of contacts with the patients $(\bar{X}=6.42, S . D .=2.75$, Range $=0-10)$. On the average, patients saw their network members two to three times a month, and talked with them slightly less often than that before the stroke. Combined contacts with network members occurred about two to three times a month, on the average. Fourteen patients (28\%) reported average network contact frequency to be only once a month or less, and five (20\%) had a high frequency of network contact on the average 
TABLE XXXI

PATIENTS ' EREQUENCY OF NETWORR CONTACTS,
TIME 1 AND TIME 2

$\frac{\text { Time } 1}{(N=50)} \quad\left(\frac{\text { Time } 2}{N=49)}\right.$

Total \# of NW Members,

Face-to-Face Contact

345

326

Average \# of NW Members,

Face-to-Face Contact

6.90

6.52

Total \# of NW Members,

Other Contacts

387

211

Average \# of NW Members,

other Contacts

6.42

4.22

Average Frequency of NW Contacts:

Face-to-Face Contacts:

ONCE A MONTH OR LESS

$13(26 \%)$

$13(26 \%)$

$19(38 \%)$

WEERLY

GREATER THAN WEEKLY

5 (10\%)

$16(33 \%)$

14 (29\%)

$15(31 \%)$

$4(8 \%)$

Other Contacts:

ONCE A MONTH OR LESS

17 (34\%)

$15(30 \%)$

9 (18\%)

WEERLY

GREATER THAN WEERLY

9 (18\%)

11 (29\%)

6 (16\%)

$14(37 \%)$

7 (18\%)

Combined Contacts:

ONCE A MONTH OR LESS

$14(288)$

$15(30 \%)$

$16(32 \%)$

WEEKLY

GREATER THAN WEERLY

5 (10\%)

$15(31 \%)$

$10(20 \%)$

$19(39 \%)$

$5(10 \%)$

* The number of valid cases for patients' other contacts at Time 2 was 38 . 
(more frequently than once a week).

Time 2 Frequency of Contact. At Time 2 , caregivers reported a total number of 393 network members with whom they had face-to-face contact $(\overline{\mathrm{X}}=7.86$, S. D. $=1.92$, range $=0-10$ ) and 328 persons with whom they had other types of interactions $(\bar{X}=6.56$, S. D. $=2.31$, range $=0-10)$. On the average, respondents saw their network members two to three times a month, and spoke with them on the telephone or had other types of contacts almost weekly. Combined contacts with network members, on the average, were greater than weekly. Eight respondents (16\%) reported average frequency of contacts of all types with network members to be monthly or less, and the same number reported average frequency of network contact to be several times per week.

Stroke patients at Time 2 had a total number of 326 network members with whom they had face-to-face contact $(\bar{X}=$ 6.52 , S. D. $=2.44$, range $=0-10)$. They talked with, or had other contact with a total of 211 network members $(\bar{x}=$ 4.22, S. D. $=3.39$, range $=0-101$. On the average, patients saw their network members two to three times a month after the stroke, and had other contact with the network slightly more frequently, but not as often as once a week. For combined contacts, frequency was two to three times per month, on the average. Again, certain respondents could be identified as having low contact lonce a month or less) or high frequency of contact (greater than weekly) 
with their networks. At Time 2, 15 respondents (318) had low frequency of contact and 5 (10\%) had high frequency of contact with non-household network members.

In sum, both caregivers and stroke patients had reasonably frequent contact with a high proportion of their intimate networks on the average, and this did not change appreciably at Time 2, except for a decrease in the number of individuals with whom they had telephone and letter contacts. Less than $20 \%$ of respondents reported low frequency of contact with their network members and 10 to 158 reported high degrees of network contact. These proportions remained about the same at Time 2 .

\section{Change in Network Contact Scores, Time 1 to Time 2}

Distribution of Time 1 to Time 2 frequency of contact change scores was examined in order to identify subgroups of patients and caregivers who had experienced significant increases or decreases in frequency of contact with members of their networks as a result of the stroke. Change data were available for 49 caregivers and 49 stroke patients. In the descriptive statistics reported below, a negative number indicates an increase in average network contact score, while a positive number indicates a decrease in average network contact score.

Face-to-Face Contacts. As a group, caregivers experienced minimal change in the average frequency of faceto-face contacts with network members between Time 1 and 
Time $2, \bar{x}=.025$, S. D. $=1.04$, range $=-2.33$ to 2.901 . In looking at the distribution of change scores for in-person network contacts, 28 of 49 caregivers (57\%) reported an increase in the average frequency of contacts, while 21 (43\%) reported a decrease in frequency of face-to-face interactions. Seven caregivers experienced decreases in average face-to-face contact frequency which were more than one S.D. from the mean, while five had change scores indicative of an increase which were more than one $S$. D. from the mean.

Stroke patients experienced a decrease in average frequency of face-to-face contacts $(\bar{x}=.137$, S. D. $=1.39$, range $=-3.94$ to 3.13 ). Examining the distribution of change scores, 18 patients (37\%) experienced an increase in the average frequency of these contacts, two showed no change, and $29(59 \%)$ showed a decrease in average frequency of face-to-face contacts. Eight patients (16\%) were more than one S. D. from the mean in experiencing a decrease in average network contact score, while 7 (14\%) had change scores indicating an increase in average contacts which were more than one S. D. from the mean.

Other Contacts. For other types of contacts, the partners reported a slight increase in average frequency score $(\bar{X}=-.192$, S. D. $=1.10$, range $=-2.57$ to 2.50$)$. Twenty-seven (55\%) experienced an increase in the average frequency of other than face-to-face contacts, 4 (8\%) 
experienced no change, and 18 (37\%) had a decrease in average frequency of other types of contacts. Eleven caregivers had an increase in average contact score which was more than one S.D. from the mean and seven had decreases which were more than one $S$. $D$ from the mean.

With regard to other types of contacts, patients experienced an increase in the average frequency of network contacts of .412 (S. D. $=1.88$, range $=-5.667$ to 5.00$)$. Half had change scores which indicated an average increase in other types of contacts, while $47 \%$ had change scores indicating a decrease on the average for these types of contacts. Only two stroke victims experienced decreases in average frequency of contacts which were more than one S. D. from the mean, while seven had change scores indicative of increases in average contacts which were more than one $S . D$. from the mean.

Combined Contacts. Caregivers' average frequency scores for all types of network contacts increased sightly over time $(\bar{x}=-.084$, S. D. $=.89$, range $=-2.24$ to 2.65$)$. Thirty respondents (61\%) had increased scores for average total network contacts, one had no change, and 18 (37\%) experienced a decrease over between Time 1 and Time 2 . Seven caregivers had change scores indicative of an increase in average contacts which were more than one $S$. D. from the mean and four had change scores more than one S. D. from the mean which showed a decrease in average frequency of all 
types of contacts.

The patients' change scores for arerage frequency of all types of network contacts indicated overall, a slight increase $(\bar{x}=-.107, \mathrm{~S} . \mathrm{D} .=1.38$, range $=-3.81$ to 3.38$)$ from Time 1 to Time 2. Twenty-four patients (49\%) had change scores which indicated an increase in average frequency of all network contacts, while for 25 respondents (51\%), change scores indicated an decrease in average frequency of combined contacts. Nine patients (18\%) had change scores indicative of increased network contact which were more than one S. D. from the mean and seven (14\%) had change scores indicating decreased combined network contacts which were more than one S. D. from the mean.

It seemed important to look separately at the distribution of change in average network contact frequency scores for those patients who were able to respond to the interview compared to those stroke victims who had communication problems which were severe enough to prevent them from participating in the interview. Non-respondent stroke patients did experience a greater mean change in average contact scores (Mean $=-.315, \mathrm{~S} . \mathrm{D} .=1.62$ ) than did respondent stroke patients (Mean $=.092$, S. D. $=1.09$ ), and a greater range of change scores, but change was not in the predicted direction of a decrease in average frequency of contact scores for those with more severe communication problems. The two groups had about the same number of 
individuals who showed increases or decreases in average contact frequency which were more than one S. D. from the mean.

Network Interconnectedness

This sample of elderly couples had highly interconnected networks, both within individual respondents' networks and across couples' networks. These data are summarized in Table XXXII. Mean density score for the caregivers at Time I was 83.7 , and for the patients was 82.9. Caregivers had an average number of within-network ties, a measure of degree of interconnectedness, of 6.3 , while the patients reported a mean number of within-network ties of 5.7 .

At Time 2, network interconnectedness had increased for both patients and caregivers, as can be seen from Table XXXII. With regard to network overlap, respondents had a mean of 3.58 network members in common at Time 1 , and this increased slightly to 3.76 at Time 2 . At Time $2,30 \%$ of the dyads had six or more network members in common, whereas $20 \%$ had reported this degree of network overlap at Time 1 .

SOCIAL SUPPORT

The concept of social support, as discussed in Chapters I - III, was considered separately from structural and interactional characteristics of the network. Respondents' reports of supportive exchanges across time in 
TABLE XXXII

INTERCONNECTEDNESS WITHIN AND BETWEEN RESPONDENTS' NETWORRS

Time 1

\section{Careqivers}

Mean Density

S. D.

Range

Mean Degree

S. D.

Range

Stroke Patients

Mean Density

$S$. D.

Range

Mean Degree

S.D.

Range
82.94

18.51

42-100

5.7

2.21

$1.0-9.0$
83.7

20.90

$31-100$

6.3

2.28

$.9-9.0$

Time 2

87.2

16.82

43-100

6.9

1.74

$3.0-9.0$

Frequency of Overlap Between Partners' Networks

$\begin{array}{rlll}0-2 & \text { NW MEMBERS } & 19(38 \%) & 20(40 \%) \\ 3-5 \text { NW MAEMBERS } & 21(42 \%) & 15(30 \%) \\ 6-10 \text { NW MEMBERS } & 10(20 \%) & 15(30 \%)\end{array}$

Deqree of Overlap

Mean \# of NW Members

$S$. D.

Range
3.58

2.33

$0-10$
86.92

16.14

36-100

5.9

2.12

$1.0-9.0$ 
four areas will be described in the following sections: instrumental support, emotional support, informational support, and socializing support. These data are summarized in Tables XXXIII and XXXIV. In addition, support provided during the stroke crisis period, marital support, overall satisfaction with support, and negative aspects of support will be discussed.

\section{Instrumental support}

Instrumental task support included assistance with household tasks and chores, and assistance during periods of illness. As can be seen in Table XXXIII, caregivers reported both giving and receiving less instrumental task assistance at Time 2 than at Time 1 . They identified a total number of 374 instances of available or actual tangible support from the close network at Time $1<\overline{\mathrm{X}}=$ $7.48)$, and 334 instances at Time $2(\bar{X}=6.68)$. Respondents reported potentially or actually providing a total number of 226 instances of instrumental support to members of their close network at Time $1(\bar{x}=4.52)$ and 176 instances at Time $2(\bar{x}=3.52)$. The amount of tangible support provided to members of the network may be underrepresented in these data, in that not all of the instrumental support eliciting questions were asked to elicit reciprocity (for example, assistance during periods of extended illness or recuperation was only asked in terms of who would provide support to the respondent). 
TABLE XXXIII

CAREGIVERS' SOCIAL SUPPORT EXCUANGES, TIME 1 AND TIME 2

Total $\underline{\bar{x}}$

Support Received

Instrumental Help

Confiding Help

Informational Help

Support Given

Instrumental Help

Confiding Help

Informational Help

Socializing support

Close Confidants

* Question not asked at Time 2 .
Time 1

Time 2

$\underline{\text { Total }} \underline{\bar{x}}$

$\begin{array}{rrrr}374 & 7.48 & 334 & 6.68 \\ 134 & 2.68 & 126 & 2.52 \\ 60 & 1.20 & 51 & 1.02\end{array}$

$\begin{array}{llll}226 & 4.52 & 176 & 3.52\end{array}$

$\begin{array}{llll}165 & 3.30 & 140 & 2.80\end{array}$

$\begin{array}{llll}100 & 2.00 & 91 & 1.82\end{array}$

$\begin{array}{llll}158 & 3.16 & 158 & 3.16\end{array}$

$145 \quad 2.90$ * 
Most caregivers named members of the close network who were providing at least some type of instrumental support. Six respondents at Time 1 and five at Time 2 named no one in the intimate network who was a source of instrumental support. All had someone to provide short-term illness support at Time 1, while three caregivers named no one to provide this type of support at Time 2. For long-term illness support, there was a more dramatic drop-off in perceived support at Time 2. Whereas at Time 1,13 caregivers saw no network member as available to provide support during a long illness, at Time 2,20 caregivers perceived themselves in this situation. This probably reflects the fact that several of the partners no longer see the stroke patient as a source of long-term illness support. Indeed, some of the concerns expressed by caregivers in responding to open-ended questions at the end of the interview expressed this theme.

Table XXXIV summarizes similar data on the amount of instrumental support received and provided by the respondent stroke patients. At Time 1, patients reported a total of 159 instances $(\bar{X}=6.12)$ of instrumental support available from the close network, and 83 instances $(\bar{x}=3.19)$ of instrumental support they would or did provide to network members. At Time 2, not unexpectedly, they perceived themselves as receiving more and providing less of this tangible type of support. They reported a total of 174 
TABLE XXXIV

PATIENTS' SOCIAL SUPPORT EXCHANGES, TIME 1 AND TIME 2 (RESPONDENT PATIENTS ONLY)

$\begin{array}{ll}\frac{\text { Time } 1}{(N=26)} & \frac{\text { Time } 2}{(N=24)} \\ \underline{N}=\underline{\text { Total }} & \text { Total } \underline{\bar{x}}\end{array}$

Support Received

Instrumental Help

Confiding Help

Informational Help
159

6.12

Support Given

Instrumental Help

Confiding Help

Informational Help

Socializing support

Close Confidants
55

25

2.12

.96

83

46

31

60

66

3.19

1.77

1.19

2. 31

70

70

66

42

74

59

13

13

7.25

2.46

.54

\section{$\bar{x}$}


instances of instrumental support received $(\bar{X}=7.25)$, and 70 instances of instrumental support provided by tinem $i \bar{x}=$ 2.92). Thus, six to eight months after the stroke, the less impaired patients still saw themselves as providing tangible assistance to close network members.

\section{Emotional Support}

Confiding support was used as the measure of emotional support over time in this study. As is summarized in Table XXXIII, caregivers perceived themselves as giving more confiding support than they received and as having about the same amount of confiding support available to them at Time 2 as at Time 1. They saw themselves, however, as providing less confiding support after the stroke compared to before. They reported a total of 134 network persons as sources of confiding support at Time $1(\bar{X}=2.68)$, and $126(\bar{X}=2.52)$ at Time 2. At Time 1 they saw themselves as providers of confiding support for 155 network members $(\bar{X}=3.30)$, whereas at Time 2 this had decreased to 140 network members $(\bar{X}=2.90)$. They named an average of 2.9 members of the close network whom they viewed as close confidants. A small number of the caregivers (8\%) reported no one in whom they could confide before the stroke and the same proportion saw themselves as not providing confiding help to any member of the network. These proportions of caregivers lacking confiding relationships did not change at Time 2 .

$$
\text { It is more difficult to interpret the confiding }
$$


relationships reported by the respondent stroke patients (see Table XXxIV). While the number of network members from whom they received confiding support remained approximately the same after the stroke, they saw themselves as providing more confiding support to their networks after the stroke compared to before. Whereas prior to the stroke they provided this type of support to 46 network members $(\bar{x}=$ 1.77), after the stroke they reported providing this type of support to 66 persons $(\bar{x}=2.75)$. This may be an artifact of the timing of the two interviews (one shortly after the stroke occurrencel, or may reflect the patients' actual perceptions of their role in providing confidant support. Only two patients reported no confiding relationships in their pre-stroke networks, and only one patient perceived himself as without any confiding relationships at Time 2 .

Patients reported an average of 2.54 network members whom they considered close confidants. Two patients named no close confidants, while almost half (46\%) had three or more close confidants.

\section{Informational support}

The provision or receipt of information and advice was not a frequently endorsed response. Both caregivers and patients reported that they provided information and advice to more persons than the number from whom they received such assistance. Caregivers reported providing slightly less informational help at Time 2 , while patients had an 
increased number of network members to whom they provided advice and information aŕter the stroke.

Socializing support

Caregivers named a total of 158 close network members with whom they socialized on a regular basis before the stroke $(\bar{x}=3.16)$, and this remained exactly the same at Time 2. Patients had 60 network members with whom they socialized regularly pre-stroke, and the total number of persons who provided this type of support increased slightly (from 60 to 70 ) at Time 2. Fifty-four percent of the caregivers at Time 1 and $58 \%$ at Time 2 named three or more persons with whom they regularly socialized. Forty-six percent of the patients at Time 1 and $38 \%$ at Time 2 had three or more network members who provided socializing support. Seven caregivers at Time 1 and eight at Time 2 socialized with none of their close network, while nine patients at Time 1 and only 2 patients at Time 2 reported that they socialized with none of their close network members.

\section{Support Reciprocity}

Four support questions involving task support, illness support, confiding, and information/advice asked about support the respondent provided to members of the network as well as support received from the network. From these data, the number of reciprocal exchanges with subjects' networks 
was determined. This information is summarized in Tables XXXV and XXXVI. In determining means and proportions of reciprocal exchanges, only the number of respondents who had reported a particular type of support received or given were considered.

Both partners and patients reported a greater number of reciprocal exchanges for short-term illness support than for other types of helping. For the caregivers, the number of reciprocal exchanges available or utilized decreased at Time 2 for all types of support. The patients reported a decreased number of reciprocal task exchanges at Time 2 , increased numbers of illness support exchanges and confiding exchanges, and the same number of informational support exchanges.

It was predicted that both caregivers and patients would experience a decrease in the degree of reciprocity within their supportive exchange networks as a result of the stroke. Further, it was anticipated that this shift in reciprocity would be in the direction of patients and caregivers being "overhelped" in the situation of coping with a chronic, disabling illness. That is, there would be fewer opportunities to reciprocate for assistance provided, as a result of the stroke. Caregivers would have less time to reciprocate in previous established ways, and patients would be less able to reciprocate. In order to explore this question of the direction of change in network reciprocity, 
TABLE XXXV

RECIPROCITY IN CAREGIVERS' HELPING NETWORRS

Time 1

Time 2

Task Support

Total \# reciprocal helps

84

56

* Mean * reciprocal helps

1.91

1.24

* Mean percent reciprocal helps

.50

.50

\section{Illness Support}

Total \# reciprocal helps

Mean * Ieciprocal helps

2.14

1.58

Mean percent reciprocal helps

.56

.47

\section{Confiding Support}

Total \# reciprocal helps

102

Mean reciprocal helps

2.17

1.88

Mean percent reciprocal helps

.53

.56

\section{Informational Support}

Total * reciprocal helps

32

25

Mean * reciprocal helps

.87

.66

Hean percent reciprocal helps

* Means and mean proportions were determined based on the number of respondents who had any instances of a particular type of support being received or provided. 
TABLE XXXVI

\section{RBCIPROCITY IN PATIBNTS' HELPING NETWORRS \\ (RESPONDENT PATIBNTS ONLY)}

Time 1

Task Support

Total *eciprocal helps

30

1.50

.48

* Mean percent reciprocal helps

\section{Illness Support}

Total *eciprocal helps

Mean \# reciprocal helps

1.70

.51

1.83

Mean percent reciprocal helpg

Time 2

\section{Confiding support}

Total * reciprocal helps

28

40

Mean \# reciprocal helps

1.17

1.74

Mean percent reciprocal helps

\section{Informational support}

Total reciprocal helps

Mean * reciprocal helps

Mean percent reciprocal helps

* Means and mean proportions were determined based on the number of respondents who had any instances of a particular type of support being received or provided. 
caregivers were grouped according to whether their networks reflected "underhelping" or "overhelping" on the part of the network, or whether there was balanced reciprocity for the different types of support. This was determined by dividing the total number of network nembers who provided a particular type of support by the total number of persons for whom the respondent provided that type of support. Thus, it is a measure of overall reciprocity, rather than reciprocity in specific dyadic relationships.

Table XXXVII summarizes this information for the caregivers. Respondents were more likely to be underhelped in the exchange of information and advice, and this persisted over time. There was an increase, however, (from 6\% to 20\%) in the proportion who reported reciprocity in the exchange of informational support at Time 2. Caregivers were more overhelped than underhelped in the provision of illness support at both Time 1 and Time 2 . The number and proportion of respondents who reported reciprocity in illness support remained about the same. About $20 \%$ of the partners reported reciprocity in their confiding relationships over time. Change in the direction of reciprocity in confiding relationships was seen, however. Whereas at Time $1,42 \%$ of caregivers reported being a confidant more than confiding in others, this had decreased to 26\% at Time 2. Thirty percent of respondents reported being overhelped in confiding relationships at Time 2 
TABLE XXXVII

DIRECTION OF RECIPROCITY IN CAREGIVERS' NETWORRS

Time 1 Time 2

Task support *

\# RECIPROCAL

\# UNDERHELPED

18

5

19

\# OVERHELPED

\# NO TASR HELP

21

6

20

5

Illness Support *

\# RECIPROCAL
\# UNDERHELPED
\# OVERHELPED
\# NO ILLNESS HELP

21

19

9

8

20

20

* NO ILLNESS HELP

0

3

Confiding support *

$\begin{array}{lrr}\text { \# RECIPROCAL } & 18 & 20 \\ \text { \# UNDERHELPED } & 21 & 13 \\ \text { \# OVERHELPED } & 8 & 15 \\ \text { \# NO CONFIDING HELP } & 3 & 2\end{array}$

\section{Informational support *}

$\begin{array}{lrl}\text { \# RECIPROCAL } & 3 & 10 \\ \text { \# UNDERHELPED } & 19 & 18 \\ \text { \# OVERHELPED } & 8 & 10 \\ \text { \# NO INFORMATIONAL HELP } & 20 & 12\end{array}$

Reciprocal helping = an equal number of network members give help to and receive help from subject.

Underhelped = subject provides more help than receives.

Overhelped $=$ subject receives more help than provides.

No helping = subject gives no and receives no help of the type specified from the close network. 
compared to $16 \%$ at Time 1 .

The patients reported little change in the diraction of reciprocity in their pre-stroke compared to their poststroke networks. This information is contained in Table XXXVIII. There was an increase in the reciprocity of confiding exchanges and a decrease in the number of patient subjects who reported overhelping in confiding relationships.

Social support During the Time of the stroke

Caregivers were asked to indicate which network members had provided specific types of stroke-related assistance during the acute hospitalization period, including tangible aid such as offering or providing rides to the hospital or assisting with household chores or errands; emotional support in the form of encouragement, empathy, and concern; and information or advice. Table XXXIX summarizes the types and amounts of support provided by the close network at the time of the stroke, as reported by the caregivers. Ninety percent identified persons who would help with transportation, although only half of the partners utilized others for transportation assistance. About 75\% of caregivers identified at least one person who was available to assist with household tasks, while only one-half named anyone who had actually provided this type of assistance. Sixty percent of the subjects reported receiving other types of tangible, or instrumental help. 
TABLE XXXVIII

DIRECTION OF RECIPROCITY IN PATIENTS' NETWORRS

$\frac{\text { Time } 1}{(N=26)} \quad\left(\frac{\text { Time } 2}{N=24)}\right.$

Task support

\# RECIPROCAL

\# UNDERHELPED

\# OVERHELPED

\# NO TASK HELP

$\begin{array}{rr}10 & 5 \\ 2 & 1 \\ 8 & 13 \\ 6 & 5\end{array}$

Illness Support

\# RECIPROCAL

\# UNDERHELPED

\# OVERHELPED

\# NO ILLNESS HELP

$\begin{array}{rr}9 & 10 \\ 2 & 1 \\ 12 & 12 \\ 3 & 1\end{array}$

Confiding support

\# RECIPROCAL

\# UNDERHELPED

\# OVERHELPED

\# NO CONFIDING HELP

$\begin{array}{rr}5 & 9 \\ 6 & 7 \\ 13 & 7 \\ 2 & 1\end{array}$

\section{Informational support}

$\begin{array}{lrr}\text { \# RECIPROCAL } & 2 & 2 \\ \text { \# UNDERHELPED } & 8 & 12 \\ \text { \# OVERHELPED } & 5 & 3 \\ \text { \# NO INFORMATIONAL HELP } & 11 & 7\end{array}$

*

Reciprocal helping = an equal number of network members give help to and receive help from subject.

Underhelped $=$ subject provides more help than receives.

Overhelped $=$ subject receives more help than provides.

No helping = subject gives no and receives no help of the type specified from the close network. 
TABLE XXXIX

SUPPORT DROVIDED DURING THE ACUTE STRORE PERIOD

(Reported by Partners, $N=50$ )

Type of support

Would provide transportation

Did provide transportation

Would assist with household tasks

Did assist with household tasks

Other types of help

Moral support

Appreciate the situation

Informational help
Total \# $\bar{x} \#$ \& of NW \# Naming

No One

$\begin{array}{rrrr}141 & 2.82 & .37 & 5 \\ 63 & 1.26 & .16 & 25 \\ 81 & 1.62 & .22 & 12 \\ 60 & 1.20 & .14 & 25 \\ 86 & 1.72 & .24 & 20 \\ 130 & 2.60 & .32 & 17 \\ 106 & 2.12 & .27 & 20 \\ 24 & .48 & .07 & 32\end{array}$


Two-thirds of respondents named at least one network member who had provided emotional aid in the form of "morzl support". Thirty-eight percent of partners reported that one-half or more of their close network had provided this type of assistance, although $34 \%$ identified no members of the close network who provided moral support. Similarly, $30 \%$ of the subjects indicated that at least half of their close network appreciated what they were going through, while $40 \%$ named no one who provided this type of emotional support.

Few members of the network provided stroke-related informational assistance or advice (a total of 24 persons, $\bar{x}$ $=.48)$. This may reflect the preference expressed by respondents in previous studies (Dunkel-schetter, 1984) to receive this type of information from health care professionals, rather than family and friends. It may also reflect that most members of the informal support network are not equipped to offer specific illness-related advice during a health crisis.

Marital support

The degree and quality of perceived support within the partner relationship itself were examined separately as an important aspect of overall social support.

Perceived Quality of the Marital Relationship. A 4item index was created which summed scores for subjects' perceptions of their overall marital happiness, the 
likelihood of confiding communication with their partners, and the relative quality of the relationship compared to other couples. Maximum score obtainable on the Marital Quality Index was 21 , and the minimum score was 4 .

overall, respondents expressed a high degree of satisfaction with their partner relationship. At Time 1 , caregivers obtained a mean score on the index of $15.89, \mathrm{~S}$. D. $=3.01$, range $=8-18$. Sixty-seven percent of the caregivers scored 17 or above. At Time 2 , the caregivers as a whole showed a slight decrease in their rating of the quality of the partner relationship, achieving a mean score of 15.22, S. D. $=3.03$, range $=7-18$ stroke victims $(N=24)$ likewise rated the quality of their partner relationship quite highly. Mean score on the Index at Time 1 for the patients was 16.45, S. D. $=1.93$, range $=11-18$. At Time 2, mean Index score was $16.54, \mathrm{~S} . \mathrm{D} .=1.98$, range $=$ 11-18.

Marital Disagreement. Subjects acknowledged little marital disagreement as it was measured in this study. They were asked to indicate the frequency of disagreement between themselves and their partner in five areas: finances, dealing with children and other relatives, making major decisions, sharing of household tasks, and leisure activities and interests. A maximum score of 15 on this 5item index would indicate frequent disagreement in all areas probed. A minimum score of 5 would indicate the subject 
felt that she or he and the partne rarely disagreed on any of the topics speciried.

At Time 1, caregivers had a mean score of 6.51 on the Marital Disagreement Index (S. D. = 1.73, range $=5-12$ ), with $38 \%$ receiving a minimum score of 5 . There was little change for the caregiver group as a whole at Time 2 . Mean Disagreement Index score was $6.09, \mathrm{~S} . \mathrm{D} .=1.62$, range $=5-$ 11. At Time 2, $54 \%$ had a minimum score of 5 on the Index.

$$
\text { Stroke victims similarly reported little marital }
$$

disagreement. Mean disagreement score at Time 1 was 6.12 (S. D. $=1.63$, range $=5-11)$ and at Time 2 was 6.13 (S. D. $=$ 1.58, range $=5-10)$. Fifty-four percent of the patients at Time 1 and $52 \%$ at Time 2 reported minimum marital disagreement scores. For the caregiver -patient partners, there was high degree of congruence in reporting of marital disagreement scores $(r=.7939, p=<.001)$.

Shared Marital Activity. Subjects were asked to indicate the frequency with which they engaged in joint activities or communication with their partner in five areas, and the number of organizations in which they had joint memberships. The resulting six-item index had a maximum possible score of 42 , and a minimum possible score of 0 . Both caregivers and patients reported a moderate degree of frequency of shared marital activity pre-stroke. The caregivers had a mean frequency score of 18.91 (S. D. = 5.18, range $=3-29$, and the patients reported a mean 
frequency score of 16.88 (S. D. $=6.22$, range $=1-27$ ) at Time 1. An equal number of partners (7) had scores on this index which were one S. D. above or below the mean. Four patients had scores at least one S. D. below the mean, and only 2 reported scores at least one $S$. D. above the mean.

At time 2, caregivers reported a reduced frequency of shared marital activity, while their partners noted an increased frequency of joint communication and activity. Mean score for the caregivers at Time 2 was 16.6 (S. D. = 6.20, range $=0-25$ ). For the patients, mean score on this index at Time 2 was 18.38 (S. D. $=5.59$, range $=7-27$ ). Ten caregivers (20\%) were at least one S. D. below the mean at Time 2. There was a strong correlation between caregiver and patient partners on this measure at Time $2(r=.7145$, p. $=\langle .001\rangle$, however, suggesting that those caregivers whose partners were unable to respond to the interview may have been the ones who accounted for the decrease in shared marital activity score for the caregivers at Time 2.

\section{Satisfaction with Network Support}

Subjects were asked to rate their overall satisfaction with both the amount and quality of social network contacts. As can be seen from the distribution of responses in Table XL, both caregivers and patients expressed high degrees of overall satisfaction with the amount and quality of their network relationships. At Time $1,74 \%$ of caregivers and $72 \%$ of stroke patients reported that they were very satisfied 
TABLE XI

RESPONDENTS' EXPRESSED SATISFACTION WITH OVERALL AMOUNT AND QUALITY OF SOCIAL NETWORK CONTACT, TIME 1 AND TIME 2

Time 1 Time 2

Satisfaction with Amount of Social Contact

Careqivers

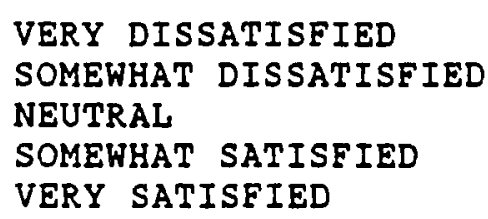

$0(0)$

$5(10)$

$3(6)$

$5(10)$

$37(74)$

$\begin{array}{rr}2 & (4) \\ 5 & (10) \\ 4 & (8) \\ 15 & (30) \\ 24 & (48)\end{array}$

Stroke Patients

VERY DISSATISEIED

SOMEWHAT DISSATISFIED

NEUTRAL

SOMEWHAT SATISFIED

VERY SATISFIED
$0(0)$

$0(0)$

$2(8)$

$5(20)$

$18(72)$
$0(0)$

$0(0)$

$4(17)$

$5(21)$

$15(62)$

\section{Satisfaction with Quality of Social contact}

Careqivers

VERY DISSATISFIED
SOMEWHAT DISSATISFIED
NEUTRAL
SOMEWHAT SATISEIED
VERY SATISFIED

VERY SATISFIED

Stroke Patients

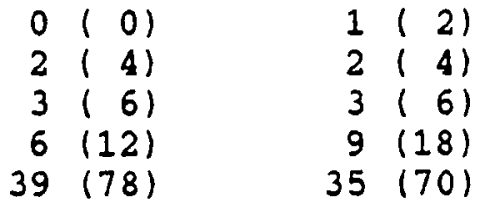

$0(0)$

$0(0)$

$2(8)$

$3(12)$

$20(80)$ $\begin{array}{rr}0 & (10) \\ 0 & (0) \\ 2 & (8) \\ 4 & (17) \\ 18 & (75)\end{array}$

* Numbers in parentheses equal percents. 
with the amount of pre-stroke contact with persons in their networks. Likewise, $78 \%$ of the caregivers and $80 \%$ of the patients were very satisfied with the quality of their social network interactions before the stroke.

At Time 2, fewer caregivers reported being very satisfied with the amount of social network contact (18\% compared to 748 at Time 1 ) or with the quality of network contact $170 \%$ versus $78 \%$ at Time 1 ). The stroke patients showed slight declines in the number and proportion who indicated that they were very satisfied with the amount and quality of network contacts since the stroke.

\section{Negative Aspects of Social Support}

Consistent with the high levels of satisfaction respondents expressed with their networks, few acknowledged any negative relationships with network members, especially at Time 1. In response to questions regarding persons who had been less help than expected, who made the situation worse, who appeared to be out to make life difficult for the respondent, or with whom the respondent didn't get along, caregivers named a total of 50 persons $(\bar{X}=1.0)$. Patients named even fewer persons, a total of 17 at Time $1(\bar{x}=.68)$. At Time 2 , caregivers named 66 negative network contacts $(\bar{x}$ $=1.32$ ) and patients reported 14 such contacts. It was perhaps difficult for the patients to acknowledge negative feelings about network relationships when they were dependent upon some of these individuals for continued and 
necessary support. Twenty-two percent of the caregivers, on the other hand, named at least one person who had been less help than they expected since the stroke had occurred.

\section{SUMMARY: INTIMATE NETWORK SOCIAL SUPPORT AND SOCIAL CONTACTS}

Overall, for this sample of patients and their partners, social support within their close or intimate networks was maintained at six to eight months following the stroke. Partners and stroke patients saw their network members about as frequently after the stroke as before. Caregivers did report receiving less instrumental support at Time 2, and slightly less confiding and informational support. They provided less confiding and instrumental support at Time 2 to members of their network. Socializing support was maintained.

Patients saw themselves as receiving increased instrumental help at Time 2 , and less informational help. They reported giving less tangible assistance to their networks, but more confiding and informational help. Socializing contacts were maintained, and even increased slightly. The patients reported fewer socializing contacts and fewer close confidants than did their partners, which is generally consistent with the available information on the social support systems of older men compared to older women (Stueve \& Fischer, 1978; Vaux, 1985).

Caregivers experienced decreased numbers of reciprocal 
support exchanges with their close networks at Time 2 across types of support, while patients reported slightly increased numbers of confiding exchanges and of reciprocal illness support. Partners also reported fewer confiding relationships in which they were the providers of confiding support. There are a number of possible explanations for these reported changes in the reciprocity characteristics of the caregivers' networks.

Respondents as a whole were very satisfied with the amount and quality of their pre-stroke social network support. For the caregivers, rating of network satisfaction decreased somewhat at time 2 , in that there were fewer who rated themselves very satisfied with the amount and quality of support available. Patients, for whatever reason, continued to see their close networks in a very positive light at Time 2. Both patients and partners reported high levels of satisfaction with their marital relationship, low levels of marital disagreement, and moderate levels of shared marital activity before the stroke. At Time 2, caregivers reported slightly less marital satisfaction and shared marital activity, while the patients reported slight increases in both aspects of their relationship. It must be kept in mind, however, that the patient group only included those who could respond to the interview questions.

Despite the fact that a descriptive analysis of the group data paints a generally optimistic picture of how this 
group of stroke patients and their partners were coping in terms of social support, analysis of score distributions indicated that there were subgroups of patients and their caregivers who were experiencing negative network changes. In Chapter VI, correlates of depressive symptoms and wellbeing at two points in time will be explored, including those related to social networks and social support. 
CORRELATES AND PREDICTORS OF DEPRESSION AND WELL-BEING:

TIME 1 AND TIME 2

This chapter will begin to test the model for this study which was presented in Chapter II. Correlates and predictors of depressive symptom scores and of positive and negative well-being will be examined, focusing on stroke severity measures, perceptions of the stroke, self-reported health, social networks and social support, and individual characteristics such as optimism and religiosity. These relationships as measured at a single point in time will be examined for both Time 1 and Time 2 . The outcome variables of depression and positive and negative well-being will be described first, for both caregivers and patients. Then, the relationship of the other variables to these outcome measures will be discussed.

Table XLI presents the means and standard deviations for the major variables at Time 1 and Time 2 for the caregivers, and Table XLII summarizes the same information for the respondent patients. Tables XLIV, XLV, XLVI, XLVII, and XLVIII present the correlations between the outcome variables and other major variables at Time 1. Following the discussion of the interrelationships among variables at Time 1, interrelationships among variables at Time 2 will be considered. Finally, the results of multiple regression analyses to assess predictors of depression and well-being 
in the caregivers at both points in time will be presented. Because the number of stroke patients who completed the outcome measures was small (26 at Time 1 and 24 at Time 2), regression analyses were not run on the patient dependent variables.

TIME 1

\section{Outcome Variables}

Depressive Symptoms: CES-D. A 28-item version of the CES-D was used in this study (Goldberg, 1981). Consequently, a cutoff score of 23 for indicating those at risk for depression was adopted rather than the usual cutoff score of 16 used with the 20-item version of the measure. At Time 1, caregivers had a mean depressive symptom score of 22.7 (S. D. $=13.32$ ) and a median score of 23 . Range of :scores was $0-55$. Twenty-two caregivers (48\%) scored above the cutoff score of 23 .

Respondent stroke patients had a mean CES-D score of 17.88 (S. D. $=11.91$ ) and a median score of 15.5. Range of scores was $0-48$. Six patients (25\%) scored above the cutoff score of 23 on the measure. It should be kept in mind that these were the least impaired stroke patients in terms of the ability to communicate.

These results for the caregivers are very similar to those obtained by williams (1985) in a recent study of physically ill elderly persons, in terms of both mean level 
TABLE XLI

MEAN VALUES FOR SELECTED CAREGIVER VARIABLES

(MAXIMUM VALUES IN PARENTHESES)

TIME 1

\section{MEAN}

CES-D 28 (84)

POSITIVE KB (9)

NEGATIVE WB (15)

BALANCE WB (7)

LOT SCORE (32)

RELIGIOSITY (14)

OBJECTIVE HEALTH

SUBJECTIVE HEALTH (5)

SEVERITY OF STRORE (5)

BARTHEL INDEX (100)

ASF SCORE (32)

CONCERN : ANOTRER CVA (5)

CONCERN: FUTURE CARE (5)

SOCIAL ACTIVITY (25)

SIZE OF OA SOCIAL NW

SIZE OF INTIMATE NW

NETWORR DEGREE (9.0)

MARITAL QUALTIY (18)

SHARED MARITAL ACTIVITY
22.70

S. D.

13.32

$4.77 \quad 2.59$

4.21

3.94

2.92

1.74

23.02

4.55

8.63

3.61

5.00

4.51

$2.76 \quad 1.21$

$3.32 \quad 1.04$

$81.81 \quad 18.81$

$13.00 \quad 6.05$

$3.86 \quad 1.40$

$3.21 \quad 1.61$

$11.02 \quad 3.26$

12.20

4.30

8.82

1.59

6.30

2.28

$15.89 \quad 3.01$

18.91

5.18

TIME 2

MEAN S. D.

$20.04 \quad 13.23$

$4.79 \quad 2.78$

$3.92 \quad 2.34$

$4.00 \quad 1.70$

$21.92 \quad 4.22$

$7.28 \quad 3.51$

$5.72 \quad 5.21$

$2.74 \quad 1.12$

$3.18 \quad .96$

$87.60 \quad 15.68$

$13.92 \quad 5.63$

$3.44 \quad 1.54$

$2.41 \quad 1.65$

$10.42 \quad 3.20$

$14.14 \quad 4.40$

$8.96 \quad 1.54$

$6.90 \quad 1.74$

$15.22 \quad 3.03$

$16.60 \quad 6.20$ 
TABLE XLII

MEAN VALUES FOR SELECTED PATIENT VARIABLES

(MAXIMUM VALUES IN PARENTHESES)

\begin{tabular}{|c|c|c|c|c|c|}
\hline & & & 1 & TIM & 2 \\
\hline & & MEAN & S. D. & MEAN & S. D. \\
\hline & CES-D $28 \quad(84)$ & 17.88 & 11.91 & 20.22 & 15.20 \\
\hline & POSITIVE WB (9) & 4.52 & 2.06 & 3.71 & 1.99 \\
\hline & NEGATIVE WB (15) & 3.48 & 2.54 & 3.67 & 3.23 \\
\hline & BALANCE WB (7) & 3.56 & 1.39 & 4.04 & 1.73 \\
\hline & LOT SCORE (32) & 20.72 & 5.10 & 19.22 & 5.80 \\
\hline & RELIGIOSITY (14) & 6.42 & 3.66 & 6.21 & 3.60 \\
\hline ઋ & OBJECTIVE HEALTH & 7.60 & 6.69 & 12.76 & 7.50 \\
\hline$\star$ & SUBJECTIVE HEALTH (5) & 2.94 & 1.13 & 3.31 & .98 \\
\hline & SEVERITY OF STRORE (5) & 2.69 & 1.05 & 2.42 & 1.02 \\
\hline & BARTHEL INDEX (100) & 92.50 & 10.11 & 95.46 & 6.89 \\
\hline & ASE SCORE (32) & 11.68 & 3.56 & 11.30 & 4.03 \\
\hline * & CONCERN: ANOTHER CVA (5) & 3.33 & 1.51 & 3.36 & 1.57 \\
\hline * & CONCERN: FUTURE CARE (5) & 2.83 & 1.63 & 2.42 & 1.74 \\
\hline * & SOCIAL ACTIVITY (25) & 10.37 & 3.97 & 9.53 & 3.78 \\
\hline & SIZE OF OA SOCIAL NW & 11.52 & 4.69 & 13.17 & 4.46 \\
\hline$\star$ & SIZE OF INTIMATE NW & 8.08 & 2.44 & 7.79 & 2.59 \\
\hline$\star$ & NETWORR DEGREE $(9.0)$ & 5.70 & 2.21 & 5.90 & 2.12 \\
\hline & MARITAL QUALITY (18) & 16.46 & 1.93 & 16.54 & 1.98 \\
\hline & SHARED MARITAL ACTIVITY & 16.88 & 6.22 & 18.38 & 5.60 \\
\hline
\end{tabular}

* Includes all stroke patients. 
of depressive symptoms and the percent of respondents who had scores indicating significant risk for depression. Williams (1985) reported a mean CES-D score for her sample of 22.5 for the 28 -item version, and that $47 \%$ had significantly high levels of depressive symptoms. The present sample of caregivers had a higher proportion which scored above the cutoff level for depression than Noh and his associates (1984) reported in a group of physically disabled individuals. Noh et al. found that $35 \%$ of their sample were at significant risk for depression. The mean depression score for this sample of caregivers was considerably higher at Time 1 than studies of large community samples of older persons have reported (Comstock and Helsing, 1976; Radloff, 1977). In a study of depressive symptoms in older women, Goldberg 11981; Goldberg et al., 1985) reported that slightly less than $10 \%$ of their sample reported high levels of depressive symptoms (above the cutoff score of 16 for the 20-item version of the CESD). The stroke patients had mean depression scores somewhat higher than those reported in large community studies, but lower than those reported by Noh and his colleagues for a sample of physically impaired individuals.

Thus, shortly after the occurrence of a stroke in their partners, we found levels of depressive symptomatology in this sample of generally physically healthy caregivers comparable to levels found in elderly individuals who had 
experienced a recent serious illness episode themselves (williams, 1985). Furthermore, they reported higher leveis of depressive symptoms than the stroke patients in the sample, although the two groups are not directly comparable because more severely communicatively impaired patients did not complete the depression measure.

Index of Psychological Well-Being (IPWB). As described in Chapter III, this is an 8-item self-report scale designed to measure mental health in adult populations. The scale is comprised of both positive and negative feeling-state items, and produces an index score (Balance Well-Being) which indicates the relative strength of positive and negative feelings. The index yields a score ranging from 1 to 7 , with 1 indicating a high level of psychological well-being. Because positive and negative well-being have been found in some respects to be relatively independent, especially when only frequency and not intensity of the feeling state is measured (Diener, 1984), positive and negative well-being scores as well as balance well-being scores are reported below, and considered separately in later analyses.

Caregivers had a mean Positive Well-Being (PSWB) score of 4.77 (S. D. $=2.59$ ) at Time 1 , with a median score of 3.37 and a range of 1-9. Mean Negative Well-Being (NGWB) score was 4.21 (S. D. $=2.92$ ), with a median of 3.81 and $a$ range of 0-11. For Balance Well-Being (BALWB), mean was 
3.94 (S.D. = 1.74 ) with a median of 4.0 and a range of $1-7$. Patients reported a mean 2 SAB score of $A .52$ (S. D. = 2.06), median $=4.38$ and range $=0-9$. Mean NGWB score was 3.48 (S. D. $=2.54$ ), with a median of 3.13 and a range of $0-$ 9. BALWB mean score for the patients was 3.56 (S. D. = 1.39), median $=3.67$ and range $=2-7$.

The overall, or BALWB scores reported by both the caregivers and patients are very similar to the results reported by Berkman (1971), who used the IPWB with a probability sample of 6,928 adults from the general population. Berkman reported a mean score for his sample of 3.77. Mean IPWB score for the caregivers in this sample was slightly higher (3.94) and for the patients slightly lower (3.56). The distribution of IPWB scores for the Berkman sample and for the caregivers and patients in this sample are compared in Table XLIII. Inspection of the table shows some differences among the samples in the distribution of scores. Caregivers in the stroke sample had a greater percentage of respondents with scores indicative of low well-being and patients had fewer respondents scoring in this range than did Berkman's sample.

\section{Level of Optimism (LOT)}

As described in Chapter III, The life orientation Test, or the LOT is an eight-item scale designed to measire dispositional optimism as a stable trait or chronic outcome expectancy. At this time, the only available norms on the 
TABLE XLIII

COMPARATIVE DISTRIBUTION OF SCORES ON THE INDEX OF PSYCHOLOGICAL WELL-BEING FOR A COMMUNITY

SAMPLE AND THE STRORE STUDY SAMPLE

\section{Percentage of Sample}

Scale

score

Berkman (1971)

Stroke Study

(Time 1)

Careqivers Patients

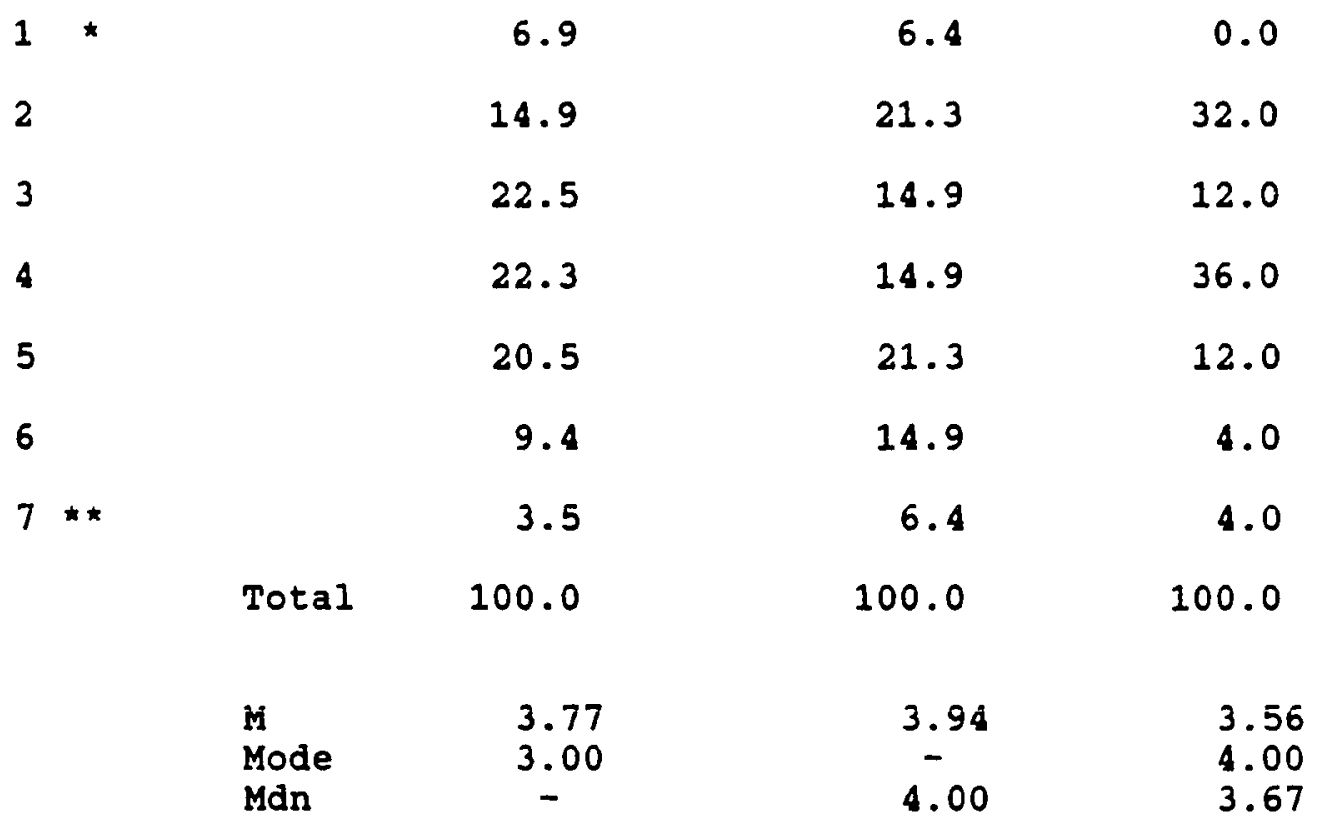

* High well-being.

* * Low well-being. 
LOT are those reported by Scheier and Carver (1983). Their studies of the instrument to date have used samples of undergraduate men and women. Scheier and Carver obtained mean LOT scores of 21.41 (S. D. $=5.22$ ) for female undergraduates and $21.03(\mathrm{~S} . \mathrm{D} .=4.56)$ for male undergraduates.

At Time 1, caregivers in this sample had a mean score on the LOT of 23.02 (S. D. $=4.55)$, a median score of 22.94 , and a range of 12 to 32 . Patients had a mean score of 20.72 (S. D. $=5.10$ ), with a median of 20.88 and a range of 13 to 30. Thus, this sample of older, primarily female caregivers scored somewhat higher and the older, primarily male stroke patients scored slightly lower on the LOT than their gender counterparts in scheier and Carver's undergraduate samples.

Distribution of scores on the LOT was also examined for the sample. Twenty percent of the caregivers scored more than one S. D. above the mean on the LOT, while $17 \%$ scored more than one S. D. below the mean. Twenty percent of the patients also scored more than one S. D. above the mean and an equal proportion scored more than one $S$. D. below the mean on the LOT.

Stroke-Related Measures

Barthel Index. The Barthel Index, previously described in Chapter III, served as a measure of reported independence in accomplishing basic activities of daily 
living, and only indirectly as a surrogate measure of the severity of the residual physical impairments resuiting from the stroke. This is, of course, because patients may perform self-care functions more or less independently than would be predicted by their level of physical disability. Caregivers and respondent patients independently rated the patient on this measure. A maximum score of 100 on the Barthel indicates independence in performing all of the specified activities of daily living.

Partners' mean rating of the patients on the Barthel at Time 1 was 81.81 (S. D. $=18.81$ ), median of 86.0 , range $=$ 45 to 100. Approximately one-third of the patients were rated 75 or below on the Index, and one-third were given maximum scores of 100 by the partners. The group of less impaired, respondent patients had a mean score of 92.5 (s. D. $=10.11$ ) on the Barthel with a median of 97.5 , range $=60$ to 100. Half of the patients gave themselves a maximum score of 100, while only one scored 75 or below.

These results were compared to Barthel Index scores for patients in the oregon and in the Three-state Community Hospital Stroke Program Surveys (Becker et al., in press). Patients in the Oregon CHSP survey, had a mean Barthel score of 65.3 (median $=75.0$ ) at the time of hospital discharge. For the three-state CHSP group, mean Barthel score at time of discharge was 61.8 (median $=65.0$ ). The patients in these surveys were older (see Chapter III) than the present 
sample of patients, however, and some had suffered more than one stroke. In a study reported by Granger, Greer, Liset, Coulombe, and O'Brien (1975), stroke patients who were discharged home from an acute care hospital had a median Barthel score of 95 , with $85 \%$ having a Barthel score greater than 60. For those who were initially transferred to a stroke rehabilitation unit and later discharged home, median Barthel score on discharge from the rehabilitation unit was 96, with a range of 47 to 100 . In conclusion, Barthel scores of this sample of patients, most of whom were living at home at the time of first interview, appear comparable to those of similar groups of patients who were discharged home either initially or after a period of rehabilitation.

Adaptive and Social Functioning Scale (ASF). This nine-item scale was adopted for use from the community Hospital Stroke Program studies (Becker et al., in press). It grossly measures some aspects of socializing, communication, and other living skills not tapped by the Barthel Index, which focuses on physical functioning. As with the Barthel, caregivers and respondent patients independently rated the patient on this scale. A minimum score of nine would indicate maximum independence in terms of the scale, while a maximum score of 32 would indicate inability to perform the scale functions since the stroke.

Caregivers gave the patients a mean rating of 13.00 (S. D. $=6.05)$, and a median score of 10.63 on the ASF. 
Range of scores was 9-29. Eight patients received a minimum score oi 9. Fatients' mean scora on this masura was 11.58 $(\mathrm{S} . \mathrm{D} .=3.56)$, median $=11.33$, range $=9-19$.

overall stroke Severity. In addition to the relatively objective measures of ADL functioning described above, patients and caregivers rated the overall severity of the stroke in terms of the amount of assistance required by the patient on a five-point scale with one indicating no problem and five indicating a very severe problem. Distribution of responses to this question were presented in Tables XVI and XVII, Chapter IV. At Time 1, Caregivers had a mean of 3.32 (S. $D=1.04$ ) on this measure, with a median score of 3.29. Patients had a mean of 2.69 (S. D. $=1.05$ ), with a median score of 2.63. Range of responses for both partners and patients was $1-5$.

In conclusion, this group of patients overall, as judged by the caregivers' responses to the Barthel Index, the Adaptive and Social Functioning Scale (ASF), and an overall severity rating scale, were less impaired and more functional than general community samples of stroke patients. They were comparable in level of ADL functioning to samples of stroke patients who eventually returned home (Granger et al., 1975).

\section{Health Status}

Objective Health Rating. Objective health rating was based on a four-item index which summed the reported number 
of pre-stroke disabilities, chronic health problems, physician visits within the past six months, and number of medications. Caregivers had a mean score of 5.0 on this index at Time 1 (S. D. = 4.51), and a median score of 3.38 . Scores ranged from 0 to 16 . Nine caregivers (18\%) had scores more than one S. D. above the mean, while six (12\%) had scores more than one $S$. D. below the mean.

Patients $(N=50)$ had a mean pre-stroke health rating of 7.60 (S. D. $=6.69)$, with a median score of 5.7 and $a$ range of $0-38$. Modal score for the patients was 11 . Five (10\%) had scores more than one S. D. above the mean, while only one had an index score more than one S. D. below the mean.

Subjective Health Rating. A single item, overall rating of health on a five-point scale from 1 = excellent to $5=$ poor was used as the measure of subjective health. Other aspects of subjective health, such as perceived changes in health status and future expectations for health status were reported in Chapter IV. The point of reference for Time 1 was pre-stroke health status.

Caregivers' self-reported health rating at Time 1 had a mean of 2.76 (S. D. = 1.21), with a median of 2.78 . Patients had an overall health rating of 2.94 (S. D. $=$ 1.13), with a median of 2.85. Distribution of scores on this item was described in Chapter IV. 
CORRELATES OF DEPRESSION AND WELL-BEING AT TIME 1

\section{Correlates of Depression}

Variables related to depression scores are presented in Table XLIV for the caregivers and in Table XLV for the patients. These variables will be discussed separately in terms of demographic variables; health-related variables; stroke-related factors; social activity, social network and social support factors; and marital relationship factors.

Demographic Factors. For the caregivers, age, income, residence variables, and pre-stroke employment status were not associated strongly with depression. Level of education was negatively correlated with depression score. Higher levels of education were associated with lower depressive symptom scores. No demographic variables were strongly correlated with depression for the respondent patients.

Other Individual Characteristics. Level of optimism was strongly and negatively related to depression score for the caregivers, and less strongly associated with depression for the patients, also in the expected direction. Interestingly, patients' level of optimism at Time 1 was correlated with caregivers' depression scores in the expected direction: higher levels of patient optimism were associated with lower caregiver depression scores. It is not an unexpected finding that these two variables would be strongly associated, with higher scores on the optimism 
TABLE XLIV

CORRELATIONS WITH CES-D FOR CAREGIVERS, TIME 1

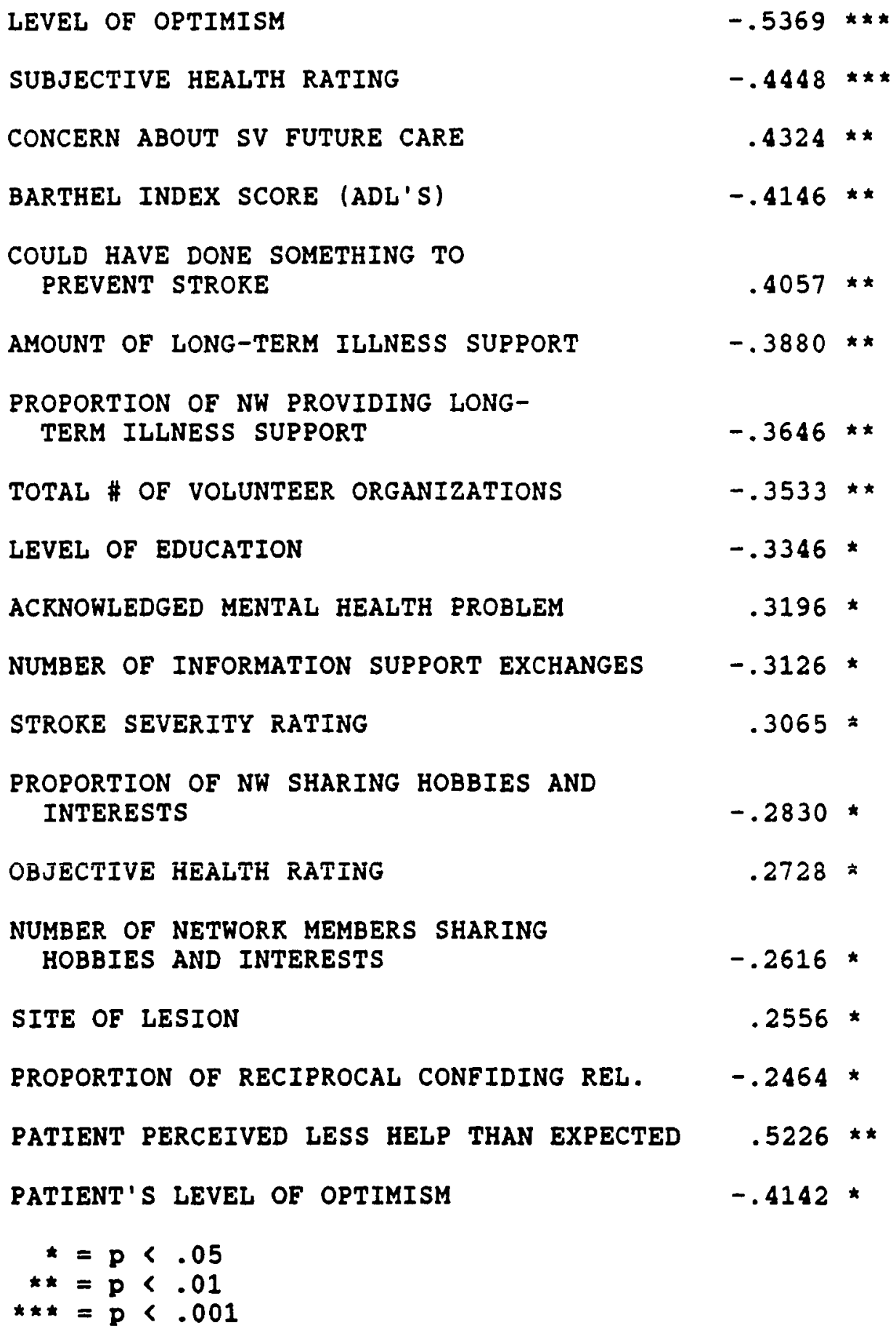


TABLE XLV

CORRELATIONS WITÄ CES-D FOR PATIENTS, TIME 1 $(N=26)$

Patient Variables

FREQUENCY OF PRE-STRORE MARITAL DISAGREEMENT

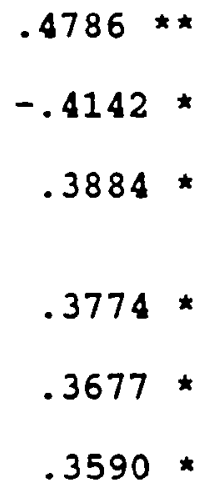

CONCERN ABOUT FUTURE CARE

PARTNER'S RATING OF MARITAL DISAGREEMENT FREQUENCY $.4072 *$

$\begin{aligned} \star & =p<.05 \\ \star \star & =p<.01\end{aligned}$ 
scale correlating significantly with lower depression scores. Degree of religiosity was not strongly related to depression scores for either patient or caregiver.

Health. Health status is frequently found to be related to levels of depression, especially in older persons (Williams, 1985). For this stroke study sample, both objective and subjective health ratings were associated with depression score. For the caregivers, subjective health rating was highly negatively correlated with depression $(\underline{\underline{r}}=$ -.44, $\mathrm{p}<.001)$. Objective health rating also correlated significantly and in the expected direction with depression. That is, higher scores on this index were associated with higher depression scores. Also as expected, acknowledgement of a pre-stroke mental health problem was associated with higher depression score.

For the patients, objective health rating as well as expectations for future health were positively associated with depression.

Stroke-Related Variables. Several stroke-related variables were associated with caregiver depression scores. Higher depression scores were related to greater concern about being able to care for the patient in the future, feeling that one could have done something to prevent the stroke, lower level of patient ADL functioning, higher stroke severity rating, and the patient having sustained other than a left hemisphere stroke. Higher caregiver 
depression levels were also associated with the patient perceiving that network members had been less helpful than expected during the stroke hospitalization and early recovery period. For the patients, feeling that one could have done something to prevent the stroke and greater concern regarding the caregiver's ability to provide care in the future were both associated with higher depression scores.

Social Activity and Social Support. Levels of depression were negatively associated for the caregivers with greater reported volunteer activity and with both the number and proportion of network members with whom they shared hobbies and interests. No pre-stroke structural network variables such as size of the network, interconnectedness, or frequency of contact were strongly associated with depression in this sample of caregivers. A number of social support variables were related to depression scores, however. Lower levels of depression were associated with a greater amount of pre-stroke perceived long-term illness support, a higher proportion of the network providing long-term illness support, a greater number of information support exchanges, and a higher proportion of reciprocal confiding relationships. Thus the amount and quality of social support were more highly associated with depression scores than were structural network variables for this sample of caregivers. No pre- 
stroke social network or support measures were significantly correlated with stroke victim depression at Time 1.

Marital Support. Marital support appeared to be more important for the patients than the caregivers in terms of depression levels. Higher levels of pre-stroke marital disagreement, both as perceived by the patient and the caregiver, were associated with higher levels of patient depression. Patients reporting a higher liklihood that their partners would share concerns and problems with them before the stroke also were more likely to report higher depression scores.

\section{Correlates of Positive Well-Being}

Table XLVI presents the correlations found with positive well-being at Time 1 for both caregivers and patients. No health-related factors, and few demographic and stroke-related factors were found to be associated with respondents' feelings of positive well-being. Rather, the important variables appeared to be aspects of overall support and marital support. These findings are discussed below.

Demographic and other Individual Characteristic Factors. As was true for depression, few strong relationships were found between demographic variables and positive well-being. For the caregivers, level of education was positively associated with positive well-being, as was age for the patients. Level of optimism, as would be 
TABLE XLVI

CORRELATIONS WITH DOSITIVE UELI-BEING FOR CAREGIVERS AND PATIENTS, TIME 1

\section{Correlations with Careqiver Variables}

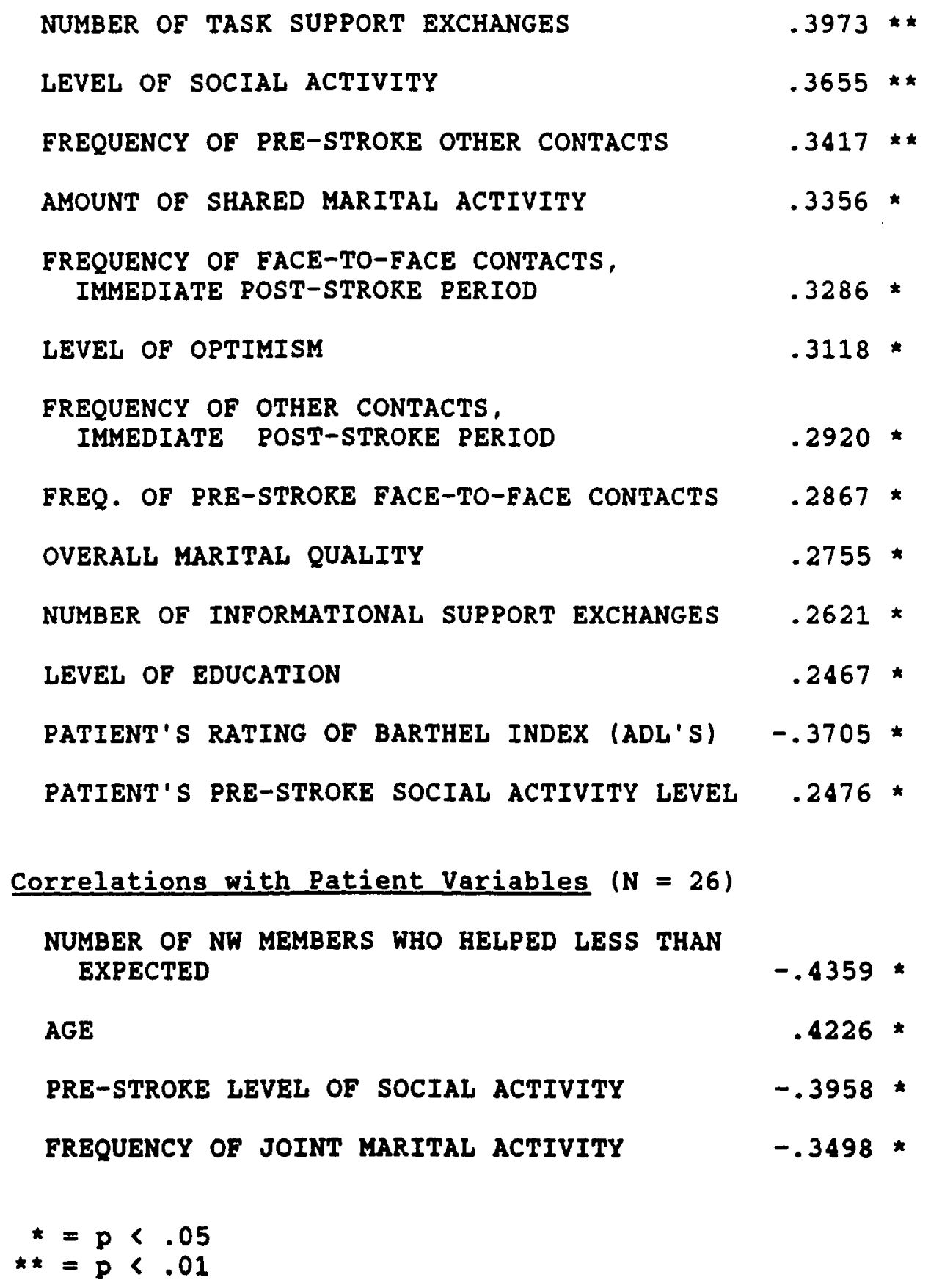


expected, was positively associated with positive well-being for the caregivers.

Stroke-Related Factors. Only one stroke-related variable was found to be strongly associated with positive well-being. Patients' ratings of their ADL's were correlated with their partners' levels of positive wellbeing, with higher levels of $A D L$ functioning reported by the patients being associated with lower PSWB scores for the caregivers. This unexpected relationship between high patient ratings and low caregiver positive well-being may reflect incongruence between patients' assessments of their level of functioning in providing self-care and their partners' assessments of their abilities.

Social Networks and Social Support. Level of prestroke social activity as well as the frequency of social network contacts both before the stroke and during the immediate post-stroke period were correlated significantly with feelings of positive well-being for the caregivers. Specific types of social support exchanges were also related to PSWB scores in the expected direction. Higher levels of task support exchanges and informationsal support exchanges in the pre-stroke network were related to higher positive well-being scores. For the patients, some negative aspects of social network support were seen. Patients who reported network members helping less than they expected during the time of early adjustment to the stroke had lower levels of 
positive well-being. Also, pre-stroke level of social activity was correlated negatively with positive well-being, suggesting that those who had been more active socially before the stroke were perhaps feeling more distress in the early post-stroke period.

Marital Support. Not unexpectedly, marital support was found to be an important correlate of positive wellbeing for both caregivers and patients. The strongest relationship to PSWB score for the caregivers was level of shared marital activity. Overall rating of marital quality also correlated significantly with positive well-being in the expected direction. For patients, greater frequency of joint marital activity before the stroke was associated with lower levels of positive well-being during the immediate post-stroke period.

\section{Correlates of Negative Well-Being}

A number of health, stroke-related, and social network and support factors were found to have a significant relationship with feelings of negative well-being. Several of these variables had similar degrees of association with depression levels. Tables XLVII and XLVIII present the correlations with negative well-being for caregivers and patients.

Demographic and other Individual Characteristics.

Higher levels of education were correlated with lower scores on the negative well-being scale for the partners. 
TABLE XLVII

CORREIATIONS HITY NEGATIVE WELL-BEING FOR CAREGIVERS, TIME :

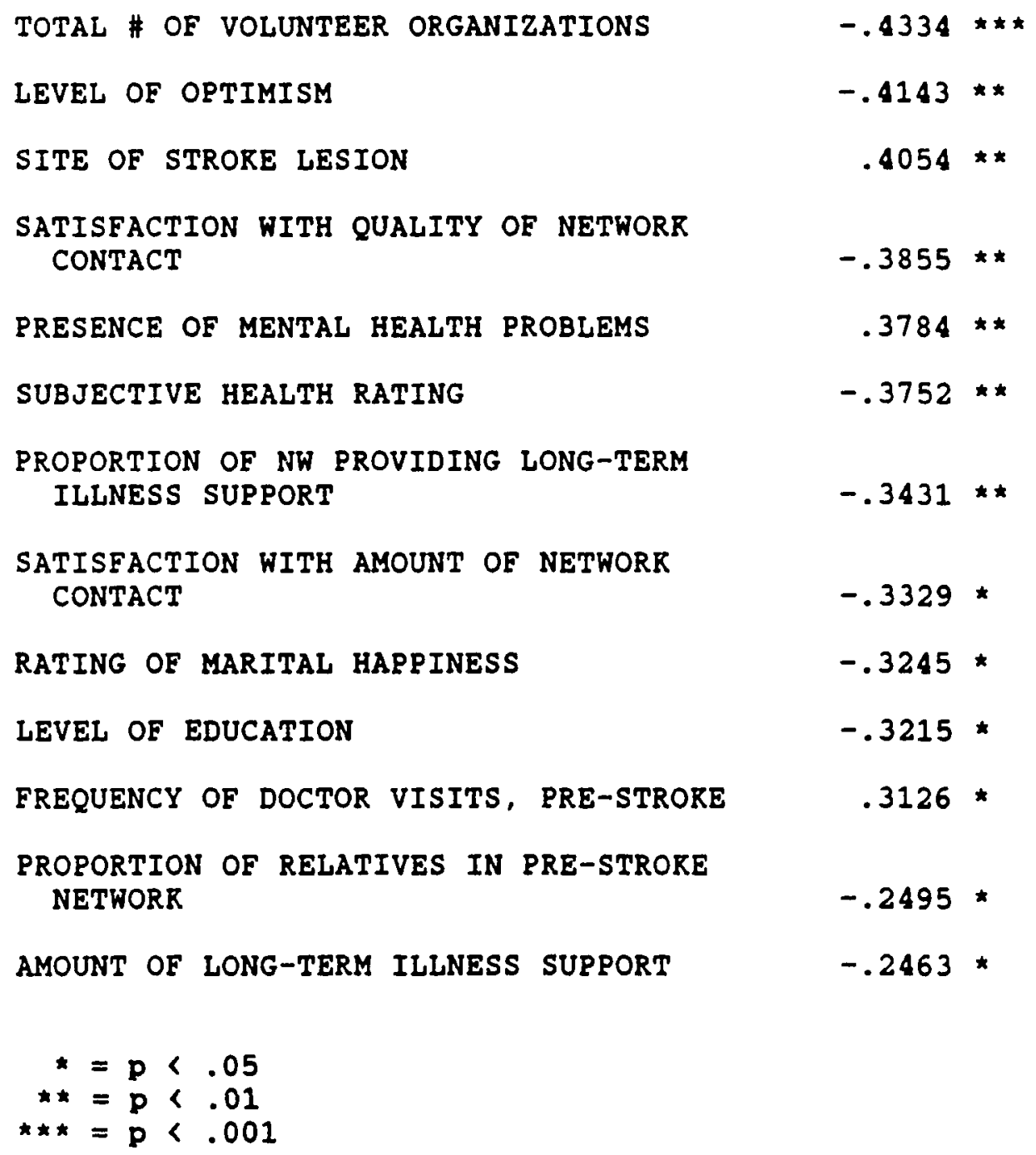


TABLE XLVIII

CORRELATIONS WITH NEGATIVE WELL-BEING FOR PATIENTS, TIME $1(N=26)$

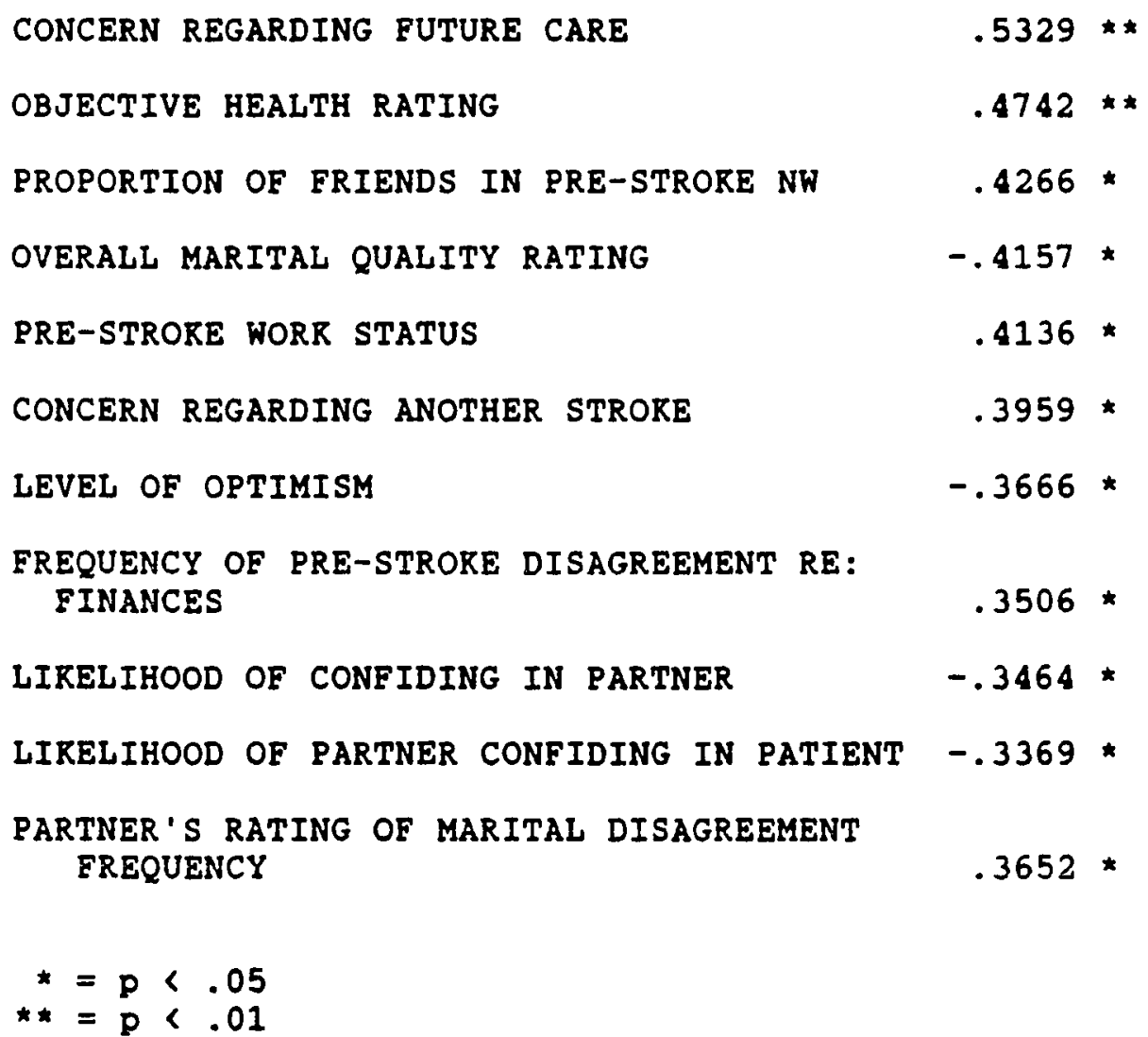

$\begin{aligned} * & =p<.05 \\ * * & =p<.01\end{aligned}$ 
Patients' pre-stroke work status was moderately related to negative well-being. Having been employed full or part-time before the stroke was associated with lower levels of negative well-being for patients. Level of optimism was strongly negatively correlated with NGWB score for the caregivers, as might be expected, and slightly less strongly for the patients.

Health. Health factors showed strong relationships with negative well-being, as they had with depression scores. Caregivers' pre-stroke subjective health ratings correlated negatively with NGWB scores (p<.01), while a higher number of pre-stroke physician visits correlated positively with negative well-being. The presence of mental health problems before the stroke also was strongly and positively related to negative well-being (p $<.01$ ). Patients' responses demonstrated a strong positive correlation between pre-stroke objective health rating and negative well-being $(p<.01)$. A greater number of disabilities, chronic health problems, physician visits, and medications before the stroke was associated with higher negative well-being scores. Thus, the strong relationship between health status and well-being described in the literature is confirmed in this study.

Stroke-Related Factors. Being more concerned about future stroke-related care and about the possibility of another stroke appeared to have negative effects on the 
patients' feelings of well-being. For the caregivers, if the site of the stroke lesion was other than in the left hemisphere, negative well-being scores were likely to be higher. One might speculate that even early in the recovery process, caregivers were aware of personality and behavioral changes in the patients with right hemisphere lesions.

Social Networks and Social Support. Again, strong associations were found between several aspects of social activity and social contact and well-being. For the partners, reporting greater amounts of time given to volunteer organizations was strongly associated with lower feelings of negative well-being $(\underline{\underline{r}}=-.43, p<.001)$, as was satisfaction with the quality $(p<.01)$ and amount $(p<.05)$ of social contact. Social support was also an important correlate of well-being. Caregivers who reported a greater amount of long-term illness support, a higher proportion of the network providing this type of tangible support, and a greater proportion of relatives in the pre-stroke network were more likely to have lower negative well-being scores. Perhaps all of these variables reflect network density, which appears to be important during an illness crisis such as a stroke. The literature confirms that it is primarily relatives who provide long-term illness support (Fischer, 1982; Litwak \& Szelenyi, 1969). With the patients, we again see the pattern of greater apparent social integration before the stroke being associated with lower levels of 
well-being in the early post-stroke period. Patients who reported a greater proportion of friends in the pre-stroke network had higher negative well-being scores at Time 1 I $\underline{\underline{r}}=$ $.43, p<.05)$

Marital Support. Marital support and the perceived quality of the relationship were strongly associated with negative well-being, in the expected direction. Caregivers who reported higher ratings of overall marital happiness were more likely to have lower NGWB scores $(\underline{\underline{r}}=-.32, \mathrm{p}<$ .05). For the patients, higher overall marital quality rating was associated with lower NGWB score $(\underline{I}=-.42, p<$ .05). The perceived likelihood of mutual confiding was associated with less negative well-being for the patients as we11. Finally, partners' perceptions of greater frequency of pre-stroke disagreement within the relationship and patients' reporting greater disagreement over finances before the stroke were both associated with higher negative well-being for the patient.

\section{Interrelationships Among Outcome Variables at Time 1}

The interrelationships between depression and various aspects of well-being, as well as the association between level of optimism and the major outcome variables were explored. Although level of optimism is viewed by some researchers as a stable coping trait (Scheier Carver, 1983), and considered as such in the model of adjustment to a stroke presented in chapter II, it seemed important to 
test this empirically in the context of a disabling illness. In addition, correlations between ceregiver well-being and patient well-being were examined in order to determine whether the partner's mood state and feeling of well-being would be seen to influence those factors in the other member of the dyad. Table XLIX summarizes the intercorrelations for the outcome measures at Time 1.

As expected, depression scores were strongly related to negative well-being and to balance well-being, the IPWB index score, for both patients and caregivers. Positive well-being, on the other hand, was not strongly related to depression score, confirming that it is a relatively independent construct.

The three well-being measures were intercorrelated as predicted. Both positive and negative well-being were strongly correlated with balance well-being, as the index score is composed of both positive and negative scores. No significant relationship was found between positive and negative well-being scores, indicating that when measured at one point in time they are tapping into different aspects of well-being.

Level of optimism was significantly related to positive well-being score at Time 1 for the caregivers but not for the patients. It was strongly related to depression and to negative well being in the expected direction. Finally, no significant relationships were found between 
TABLE XLIX

IMTERCORRELATIONS AMONG OUTCOME YARIABLES AT TIME 1

\begin{tabular}{|c|c|c|c|c|c|c|c|c|c|c|}
\hline YARIABLE & CES-D & POSITIVE & $\underset{\text { WB }}{\text { NEGATIVE }}$ & $\underset{\mathbf{W B}}{\text { BALANCE }}$ & LOT & $\begin{array}{l}\text { PATIENTS' } \\
\text { CES-D }\end{array}$ & $\begin{array}{l}\text { PATIENTS' } \\
\text { POSITIVE } \\
\text { WB }\end{array}$ & $\begin{array}{l}\text { PATIENTS' } \\
\text { MEGATIVE } \\
\text { WB }\end{array}$ & $\begin{array}{l}\text { PATIENTS' } \\
\text { BALANCE } \\
\text { WB }\end{array}$ & $\begin{array}{l}\text { PATIENTS' } \\
\text { LOT } \\
\text { SCORE }\end{array}$ \\
\hline CES-D & 1.00 & & & & & & & & & \\
\hline $\begin{array}{l}\text { POSITIVE } \\
\text { HELL-BEING }\end{array}$ & -.1727 & 1.00 & & & & & & & & \\
\hline $\begin{array}{l}\text { MEGATIVE } \\
\text { MELL-BEING }\end{array}$ & .5821 tה & $-.2694 \star$ & 1.00 & & & & & & & \\
\hline $\begin{array}{l}\text { BALANCE } \\
\text { WELL-BEING }\end{array}$ & $.4120 \star \star$ & $-.7477 \pi t *$ & $.7659 \star \Delta \star$ & 1.00 & & & & & & \\
\hline LOT SCORE & $-.5369 t$ & $.3118^{\star}$ & $-.4143 t \star$ & $-.3853 * x$ & 1.00 & & & & & \\
\hline $\begin{array}{l}\text { PATIENTS' } \\
\text { CES-D }\end{array}$ & 0967 & -.1211 & .1524 & .2043 & .0232 & 1.00 & & & & \\
\hline $\begin{array}{l}\text { PATIENTS' } \\
\text { POSITIVE WB }\end{array}$ & .0884 & .1836 & .1496 & -.0276 & -.0288 & -.2285 & 1.00 & & & \\
\hline $\begin{array}{l}\text { PATIENTS' } \\
\text { MEGATIVE WB }\end{array}$ & .1079 & .0823 & ו11. & -.0254 & -.1464 & $.5327 \star \star$ & .1414 & 1.00 & & \\
\hline $\begin{array}{l}\text { PATIENTS' } \\
\text { BALANCE WB }\end{array}$ & .0074 & -.1142 & .1662 & .1132 & -.1017 & $.6421 * \hbar$ & $-.5427 \hbar \hbar$ & $.6788^{\star x t \star}$ & 1.00 & \\
\hline $\begin{array}{l}\text { PATIENTS' } \\
\text { LOT SCORE }\end{array}$ & -.2888 & -.1083 & -.1106 & .0171 & .3837* & $-.4142 \star$ & .1412 & $-.3666^{\star}$ & $-.3602 \star$ & 1.00 \\
\hline
\end{tabular}


patient and caregiver outcome measures, although the relationship between caregiver and patient positive weilbeing approached significance $(\underline{r}=.31, p=.075)$.

\section{Effects of Confounding Variables.}

Gender. Because a small number of partners were male (9) and a small number of patients were female, the effect of gender was of concern. Especially with regard to social network relationships and social support, researchers have reported gender differences (Fischer, 1982, Stueve \& Fisher, 1978, Depner \& Ingersoll-Dayton, 1985). Numerous bivariate correlations revealed no significant relationships between any of the outcome measures and the gender variable. Because the number of male caregivers and female patients were too small to carry out meaningful intergroup comparisons, the regression analyses described in the next section of the chapter were run both including and excluding the male caregivers. The effects of excluding the male caregivers from the regression analyses will be discussed in the section describing regression analysis results.

other stressful Life Events. Fifty percent of the caregivers reported at least one major negative life event in the year before the stroke, and $25 \%$ reported at least one positive life event during that period. Forty percent of the patients had experienced at least one negative life event, and $10 \%$ had experienced one major positive life event. Neither positive nor negative life events were 
associated significantly with any of the Time 1 outcome measures, however. It may be that the occurrence of a major health crisis such as a stroke diminishes the possible effects of other life events.

jocial Desirability. In survey research, especially with an elderly cohort and in face-to-face interviews, one must be concerned about the possibility of subjects giving what they perceive as socially desirable responses. Furthermore, many of the sensitive questions in this research protocol might be viewed as having a high probability of eliciting socially desirable responses. For this reason, a six-item Social Desirability scale adopted from that of Crowne and Marlowe (1960) was included in the interview protocol as a checklist item which subjects completed on their own. Subjects responded to each statement as to whether it was true or false in describing them. A maximum score of 6 on the scale would indicate a high level of socially desirable responding. Investigation of the internal consistency of the scale with this sample yielded unacceptably low alpha levels. Caregivers had a mean score of 3.23 on the scale at Time 1 , with an S. D. of 1.54. At Time 2 , the mean score was $3.33, \mathrm{~S} . \mathrm{D} .=1.73$. Patients had a mean score of 3.00 (S. D. $=1.51$ ) at Time 1 and 3.26 (S. D. $=1.32$ ) at Time 2. Correlational associations of the Social Desirability scale with the outcome measures were minimal. Because of problems with its 
internal consistency and its generally weak relationships to the outcome measuras, the Social Desirability gcale yas not included in further analyses.

\section{REGRESSION ANALYSES: TIME 1}

Combined Effect of Selected Factors on Depression

Hiexarchical regression analyses, both with and without male caregivers included, were carried out, regressing depression score on selected caregiver variables. Variables selected for inclusion in the regression equation were chosen on the basis of their potential importance to the model presented in Chapter II as well as the strength of their relationship to depression score. A further consideration was to enter those variables with possible confounding effects in interpreting the contributions of social network and social support variables into the regression equation first, thus controlling for the effects of these variables. For example, subjective health rating and Barthel Index score were entered into the equation before the social support variables. Hierarchical, rather than step-wise regression was selected because the direction of causality was assumed to be in the direction of health, stroke severity, and pre-stroke social support influencing depression score during the early post-stroke period.

Prior to conducting the regression analyses, intercorrelations among the independent variables were 
examined to check for the presence of significant co-linearity. Variables selected for inclusion in the regression equation with depression score were subjective health rating, Barthel Index score, concern regarding future care, proportion of the network providing instrumental support before the stroke, and the proportion of reciprocal confiding relationships. These variables were only weakly or modestly correlated with each other. The strongest correlation was between subjective health rating and concern regarding future care of the patient $(\underline{\underline{x}}=-.33)$. Variables were entered into the regression equation in the order specified above.

Hierarchical Regression Results: All Careqivers. Table L presents the results of the hierarchical regression analysis when all caregivers were included. The independent variables together resulted in a regression equation with $\underline{R}$ $=69$, accounting for slightly less than half of the variance in depression score $\left(\underline{R}^{2}=.47\right)$. Pre-stroke subjective health rating accounted for almost half of the explained variance with an $\underline{R}^{2}$ Change of .21. Barthel score accounted for an additional $13.9 \%$ of the variance. Concern regarding future ability to care for the patient explained 5.7\% of the variance in depression score, while the two social support measures explained an additional $6.7 \%$ of the variance. Pre-stroke instrumental support accounted for $3.2 \%$ and the proportion of reciprocal confiding 
Table L

HIERARCHICAL REGRESSION FOR CES-D, TIME 1: ALL CAREGIVERS INCLUDED

Dependent Variable CES-D

\begin{tabular}{|c|c|c|c|c|c|}
\hline & Multiple R & $\mathrm{R}^{2}$ & $\mathbf{R}^{2}$ Change & S1mple R & Beta \\
\hline Subjective Health Rating & .455 & .207 & .207 & -.455 & -0.265 \\
\hline Barthel Index Score & .588 & .346 & .139 & -.426 & -0.397 \\
\hline Concern regarding future care & .635 & .403 & .057 & .432 & 0.202 \\
\hline $\begin{array}{l}\text { Proportion Instrumental } \\
\text { Task Assistance }\end{array}$ & .659 & .435 & .032 & -.314 & -0.165 \\
\hline Proportion Reciprocal Confiding & .685 & .470 & .035 & -.211 & -0.194 \\
\hline
\end{tabular}


relationships for $3.5 \%$ of the variance in depression score. in summary, for this group of caregivers, their subjective assessment of their own health prior to the stroke together with their assessment of the patient's level of functional independence in activities of daily living were the most important factors in accounting for depression score levels. Concern about ability to care for the patient in the future and social support elements of the pre-stroke network contributed about equally to explaining the level of depressive symptomatology. A more positive assessment of one's health together with a more positive assessment of the patient's abilities to manage his self-care would work together to decrease the concern about ability to care for the patient in the future. Caregivers' expectations that instrumental support and confiding support would be available in the future because they were present in the pre-stroke network might further reduce the likelihood of depression in the early weeks of coping with the occurrence of a stroke.

Hierarchical Regression Results: Female Caregivers. When male caregivers were excluded from the regression analysis, an equation with $\underline{R}=.74$ resulted, with $54.9 \%$ of the variance in depression scores explained. For the female caregivers, subjective health accounted for $21.6 \%$ of the variance in depression, Barthel score accounted for 14.7\%, concern about being able to care for the patient in the 
future explained 7.98, and social support factors $10.6 \%$. These data are summarized in Table LI. Social support factors accounted for most of the increased variance explained when only female caregivers were considered. This result is consistent with the literature on the relatively greater importance placed upon the wider network by older women as compared to older men, who tend to depend more on their spouses for support.

\section{Combined Effect of Selected Factors on Positive Well-Being}

The same procedures as those described for depression were used for establishing the variables to be included in hierarchical regression analyses in which positive wellbeing was the dependent variable. The variables selected for inclusion and their order of entry into the regression equation were as follows: The patient's Barthel Index score, level of pre-stroke social activity, reported quality of the marital relationship, level of pre-stroke shared marital activity, and the total number of task exchanges in the pre-stroke network. Inspection of the intercorrelations between these variables revealed that some were moderately correlated. The strongest correlation was between instrumental task exchanges and level of social activity ( $\underline{\underline{r}}$ $=.581$. Correlation between level of shared marital activity and rating of marital quality was $\underline{r}=.46$. Hierarchical Regression Results: All Careqivers. Together the variables resulted in a regression equation 
Table LI

HIERARCHICAL REGRESSION FOR CES-D, TIME 1: FEMALE CAREGIVERS ONLY

Dependent Varlable CES-D

\begin{tabular}{lccccc} 
& Multiple R & $\mathrm{R}^{2}$ & $\mathrm{R}^{2}$ Change & S1mple R & Beta \\
\cline { 2 - 6 } & .464 & .216 & .216 & -.464 & -0.247 \\
Subjective Health Rating & .602 & .362 & .147 & -.440 & -0.397 \\
Barthel Index Score & .664 & .442 & .079 & .518 & 0.216 \\
Concern regarding future care & & & & -.370 & -0.199 \\
$\begin{array}{l}\text { Proportion Instrumental } \\
\quad \text { Task Assistance }\end{array}$ & .704 & .496 & .055 & -.302 & -0.246 \\
Proportion Reciprocal Confiding & .741 & .549 & .052 & -.302
\end{tabular}


with $\underline{R}=.61$, accounting for $36.8 \%$ of the variance. Data are presented in Table III. Patients' functional ADL abilities score explained almost none of the variance in caregiver positive well-being. Level of pre-stroke social activity accounted for $15.7 \%$ of the variability in PSWB score, and was the strongest early predictor of partner well-being. Rating of overall quality of the marital relationship accounted for less of the variance in positive well-being (2.1\%) than did the amount of shared marital activity before the stroke (9.8\%) or the number of prestroke network task exchanges (9.3\%). Overall, the measures of the partner relationship and of social activity and support explained almost $40 \%$ of the variance in well-being level. These results appear to confirm that a sense of well-being is strongly related to level of sorial integration and social support.

Hierarchical Regression Results: Female Caregivers. Exclusion of male caregivers from the regression analysis resulted in a regression equation with slightly less predictive power, $\underline{R}=.60$, accounting for $35.6 \%$ of the variance in positive well-being. These data are summarized in Table LIII. Compared to regression results when male caregivers were included, level of pre-stroke social activity becomes slightly less important and level of shared marital activity as well as the amount of pre-stroke task support become slightly more important. 
TABLE LII

HIERARCHICAL REGRESSION FOR POSITIVE WEILL-BEING, TIME 1: ALL CAREGIVERS

\begin{tabular}{|c|c|c|c|c|c|}
\hline \multirow[t]{2}{*}{ Dependent Varlable } & \multirow[b]{2}{*}{ MULTIPLE $\mathrm{R}$} & \multirow[b]{2}{*}{$\mathrm{R}^{2}$} & \multirow[b]{2}{*}{ CHANGE } & \multirow[b]{2}{*}{ SIMPLE $R$} & \multirow[b]{2}{*}{ BETA } \\
\hline & & & & & \\
\hline Barthel Index & .001 & .000 & .000 & -.001 & -0.115 \\
\hline $\begin{array}{l}\text { Level of Social Activity } \\
\text { (Pre-Stroke) }\end{array}$ & .397 & .157 & .157 & .393 & 0.049 \\
\hline $\begin{array}{l}\text { Rat1ng of Marital Quality } \\
\text { (Pre-Stroke) }\end{array}$ & .422 & .178 & .021 & .276 & 0.127 \\
\hline $\begin{array}{l}\text { Level of Shared Marital Activity } \\
\text { (Pre-Stroke) }\end{array}$ & .525 & .276 & .098 & .465 & 0.353 \\
\hline $\begin{array}{l}\text { Number of NW Task Exchanges } \\
\text { (Pre-Stroke) }\end{array}$ & .607 & .368 & .093 & .430 & 0.349 \\
\hline
\end{tabular}


TABLE LIII

HIERARCHICAL REGRESSION FOR POSITIVE WELL-BEING, TIME 1: FEMALE CAREGIVERS ONLY

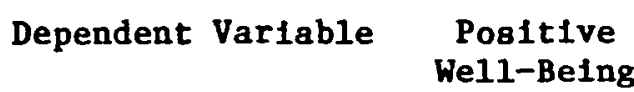

We11-Being

Barthe1 Index

Level of Social Activity (Pre-Stroke)

Rating of Marital Quality (Pre-Stroke)

Level of Shared Marital Activity (Pre-Stroke)

.502

.597

.252

.356

$\mathrm{R}^{2}$ CHANGE

SIMPLE R

BETA

Number of NW Task Exchanges

(Pre-Stroke)

$\begin{array}{lllll}.017 & .000 & .000 & -.017 & 0.131 \\ .322 & .104 & .103 & .320 & 0.004 \\ .359 & .129 & .025 & .244 & 0.137 \\ .502 & .252 & .123 & .440 & 0.354 \\ .597 & .356 & .105 & .463 & 0.375\end{array}$


Combined Effect of Selected Factors on Negative Well-Being

$$
\text { Variables selected to be included in the regression }
$$
equation with negative well-being were: subjective health rating, self-reported marital happiness, satisfaction with the quality of social network contacts, and the proportion of network members providing instrumental support help. All of these variables referred to the situation before the stroke. In addition, site of the stroke lesion was entered into the regression as a dummy variable at the second step. None of these variables correlated very substantially with each other, with the exception of the dummy variables for left and right hemisphere lesion site. These correlated very highly with each other, in a negative direction $(\underline{\underline{r}}=$ -.82). Consequently, the dummy variable for right hemisphere site of lesion was not entered into the regression equation. The strongest other correlation was between subjective health rating and instrumental support, $\underline{r}$ $=.351$.

Hierarchical Regression: All Caregivers. The combined variables resulted in a regression equation with $\underline{R}=.68$. accounting for $45.6 \%$ of the variance in negative well-being. Table LIV presents the summary data for this regression equation. Subjective health explained $17.3 \%$ of the variance in negative well-being, with the existence of a left hemisphere lesion explaining almost as much, 15.8\%. The relationship of left hemisphere site of lesion to negative 
TABLE LIV

HIERARCHICAL REGRESSION FOR NEGATIVE WELL-BEING, TIME 1: ALL CAREGIVERS INCLUDED

Dependent Variable $\begin{gathered}\text { Negative } \\ \text { Well-Being }\end{gathered}$

Subjective Health Rating (Pre-Stroke)

Left Hemisphere Lesion $\left(D_{1}\right)$

Other than RIght or Left

Hemisphere Lesion ( $\left.D_{3}\right)$

Rating of Marital Happiness (Pre-Stroke)

Satisfaction with Quality of NW Contacts (Pre-Stroke)

Instrumental Help-Proportion of NW (Pre-Stroke)

\begin{tabular}{ccccc} 
MULTIPLE R & $\mathrm{R}^{2}$ & $\mathrm{R}^{2}$ CHANGE & SIMPLE R & BETA \\
\hline .417 & .173 & .173 & -.417 & -0.222 \\
.576 & .331 & .158 & -.523 & -0.333 \\
.576 & .331 & .000 & .212 & -0.073 \\
.609 & .370 & .039 & -.324 & -0.236 \\
.675 & .456 & .086 & -.391 & -0.317 \\
.676 & .456 & .000 & -.245 & -0.020
\end{tabular}


well-being was a negative one, however. These results appear to suggest that the existence of a right hemisphere lesion correlates positively with negative well-being for the caregiver, since all types of strokes other than right or left hemisphere (total $=5$ ) were included in dummy variable 3 (D3), which accounted for none of the variance in negative well-being. Subjective marital happiness explained an additional 3.98 of the variance, while satisfaction with the quality of network contacts explained 8.6\%. The degree of instrumental task assistance within the network accounted for almost none of the variance in negative well-being.

\section{Hierarchical Regression: Female careqivers. The} regression equation for only female caregivers resulted in an improvement in prediction of negative well-being, $\underline{R}=$ $.72, \underline{R}^{2}=.51$. A summary of these regression results is presented in Table LV. Satisfaction with quality of social network contact before the stroke accounted for most of the improvement in explaining the variance in negative wellbeing for the female caregivers. It accounted for $14.1 \%$ of the variance compared to $8.6 \%$ when all caregivers were included. This again is consistent with the notion that network contacts may be more important to feelings of wellbeing for older women compared to older men. 
TABLE LV

HIERARCHICAL REGRESSION FOR NEGATIVE WELL-BEING, TIME 1: FEMALE CAREGIVERS ONLY

\section{Dependent Varlable Negative We11-Being}

Subjective Health Rating (Pre-Stroke)

Left Hemisphere Lesion $\left(D_{1}\right)$

Other than Right or Left

Hemisphere Lesion $\left(D_{3}\right)$

Rating of Marital Happiness (Pre-Stroke)

Satisfaction with Quality of NW Contacts (Pre-Stroke)

\begin{tabular}{ccccc} 
MULTIPLE R & $\mathrm{R}^{2}$ & $\mathrm{R}^{2}$ CHANGE & SIMPLE R & BETA \\
\hline .416 & .173 & .173 & -.416 & -0.253 \\
.579 & .335 & .162 & -.534 & -0.258 \\
.579 & .335 & .000 & .178 & -0.022 \\
.608 & .370 & .034 & -.291 & -0.274 \\
& & & & \\
.715 & .511 & .141 & -.466 & -0.411
\end{tabular}


TIME 2

\section{Outcome Variables}

Depressive symptoms: CES-D. At Time 2, Caregivers had a mean score of 20.04 on the CES-D, S. D. $=13.23$. Median score was 18.25 and range was 0-54. Twenty-one caregivers (45\%) were above the cutoff score of 23 . Patients had a mean score of 20.22 , S. D. $=15.20$, with a median score of 16.25 , range $=3-68$. Twenty-three percent scored above the cutoff of 23. Caregivers' depression scores decreased overall, while patients' CES-D scores increased. Distribution in CES-D change scores over time will be considered in Chapter VII.

IPWB. Positive well-being scores did not change for the caregivers as a group. Mean PSWB score at Time 2 was 4.79, S. D. = 2.78. Negative well-being scores decreased slightly, the mean at Time 2 being $3.92, S . D .=2.34$. Oyerall IPWB score remained about the same; the mean at Time 2 was 4.00, S. D. $=1.70$.

For the patients, the mean PSWB score was 3.71 , S. D. $=1.99$ and the mean NGWB score was $3.67, S . D .=3.23$. IRWB score had a mean value of 4.04, S. D. $=1.73$. Thus, for the patients at Time 2 positive well-being score overall decreased, while negative well-being and IPWB balance scores increased. The distribution of the IPWB change scores will be examined in Chapter VII. 
Level of Optimism

Both the caregivers and patients' scores on the ior declined slightly at Time 2. The mean LOT score for the caregivers was $21.92, \mathrm{~s} . \mathrm{d} .=\mathbf{4 2 2}$. For the patients, the mean LOT score was 19.22 , S. D. $=5.80$. Distribution of change in LOT scores will be discussed in Chapter VII.

\section{Stroke-Related Measures}

Barthel Index. At Time 2, caregivers gave their partners a mean Barthel Index rating of 87.6, S. D. $=15.68$. Patients had a mean Barthel rating of $95.46, \mathrm{~S} . \mathrm{D} .=6.89$. Caregivers saw improvement overall in the ADL functioning of their spouses over time, and the less communicatively impaired patients saw themselves as improved in functional independence with time. Forty percent of the patients received maximum ratings of 100 on the Barthel from the caregivers at Time 2. These improvements with time in ADL functioning are consistent with reports from other stroke studies (Becker et al., in press; Granger et al., 1975).

Adaptive and Social Functioning Scale (ASE). Patients had a mean score on the ASF of 11.30 at Time $2, S . D .=$ 4.03. Caregivers gave the patients a mean score of 13.92 , S. D. $=5.63$. The respondent patients saw themselves as slightly improved at Time 2 on this measure, while the mean score caregivers gave the patients changed slightly in the direction of less functional performance.

Overall stroke Severity. At Time 2, caregivers had a 
mean score of $3.18, S . D_{0}=.96$ in rating the severity of the patient's physical problems. Respondent patients had a mean rating of $2.42, S . D .=1.02$. These perceptions of the stroke-related physical problems being less severe at Time 2 are consistent with the overall higher ratings on the Barthel Index.

Caregiver Burden Scale (CBS). This 14-item scale was administered only to the caregivers, and only at Time 2. Maximum score on the scale was 56, with each item being scored from 0 to 4, being indicative of a high degree of perceived burden. Partners had an overall mean score on the CBS of 15.92 , S. D. $=9.67$. Median score was 14.5, with a range of 1-39. Caregivers were divided equally into those who appeared to have minimal feelings of burden, those experiencing moderate feelings of burden, and those whose scores indicated high levels of perceived burden. One-third scored more than one S. D. below the mean, and one-third scored more than one S. D. above the mean.

Personality and Behavioral Changes in the Patients. At Time 2, caregivers also completed a 19-item checklist which contained a number of behaviors and personality characteristics which the literature indicates are associated with having had a stroke. The list contained statements with positive as well as negative connotations, in order to reduce response bias. Six items had a positive connotation, and thirteen negative. Caregivers indicated 
whether the frequency of occurrence of each behavior or characteristic was less than, more than, or about the same as before the stroke. Caregivers reported a mean of $\mathbf{4 . 3 2}$ (S. D. = 3.24) negative behaviors occurring more frequently since the stroke and a mean of 2.09 (S. D. $=2.76$ ) negative behaviors occurring less frequently since the stroke. The frequency raige in each case was $0-11$. They reported a mean of .81 positive behaviors occurring more frequently (S. D. $=1.38$ ) and 1.79 positive behaviors occurring less frequently (S. D. $=1.65$ ). The frequercy range was $0-6$ in each case. Finally, the caregivers reported a mean of 10.11 behaviors or characteristics which occurred with about the same frequency as before the stroke $(\mathrm{S}$. D. $=4.53)$. Range was 0-19. Some of these behavioral change variables correlated significantly with the outcome measures, and will be discussed in the sections on correlates of depression and well-being at Time 2 .

\section{Health Status}

Objective Health. Mean score for caregivers on the objective health scale at Time 2 was $5.72, \mathrm{~S} . \mathrm{D} .=5.21$. Median was 4.33 , with a range of $0-20$. This represents a slight increase in negative health indicators for the caregivers.

Patients as a group had a mean score on the objective health index at Time 2 of 12.76 , S. D. $=7.50$. Median score was 11.5 , and range was 3-38. This was, not surprisingly, a 
significant increase in negative health indicators for the patients $(\underline{t}=-4.18, p \leq .001$, two-tailed).

Subjective Health. Overall subjective health rating for the caregivers at Time 2 had a mean of $2.74, S . D .=$ 1.12. Patients as a group rated their overall health as worse, mean $=3.31, \mathrm{~S} . \mathrm{D} .=.98$. While caregivers reported no change in their health over time, the change in overall health rating by the patients just failed to reach significance $(\underline{t}=-1.89, p=.065$, two-tailed).

CORRELATES OF DEPRESSION AND WELL-BEING AT TIME 2

\section{Correlates of Depression}

Table LVI and Table LVII present the correlates of depression at Time 2 for the caregivers and patients respectively. Demographic factors and other individual characteristics, health variables, stroke-related factors, social network and support, and marital relationship factors will be discussed separately in terms of their relationship to depression.

Demographic and other Individual Characteristics. Several related demographic factors correlated significantly with depression scores for the patients and the caregivers at Time 2. Being younger and having experienced a change in employment status as a result of the stroke were associated with higher depression scores. As reported in chapter IV, a number of patients who had been employed full time before 
TABLE LVI

CORRELATIONS WITU CES-D FOR CAREGIVERS, TIME 2

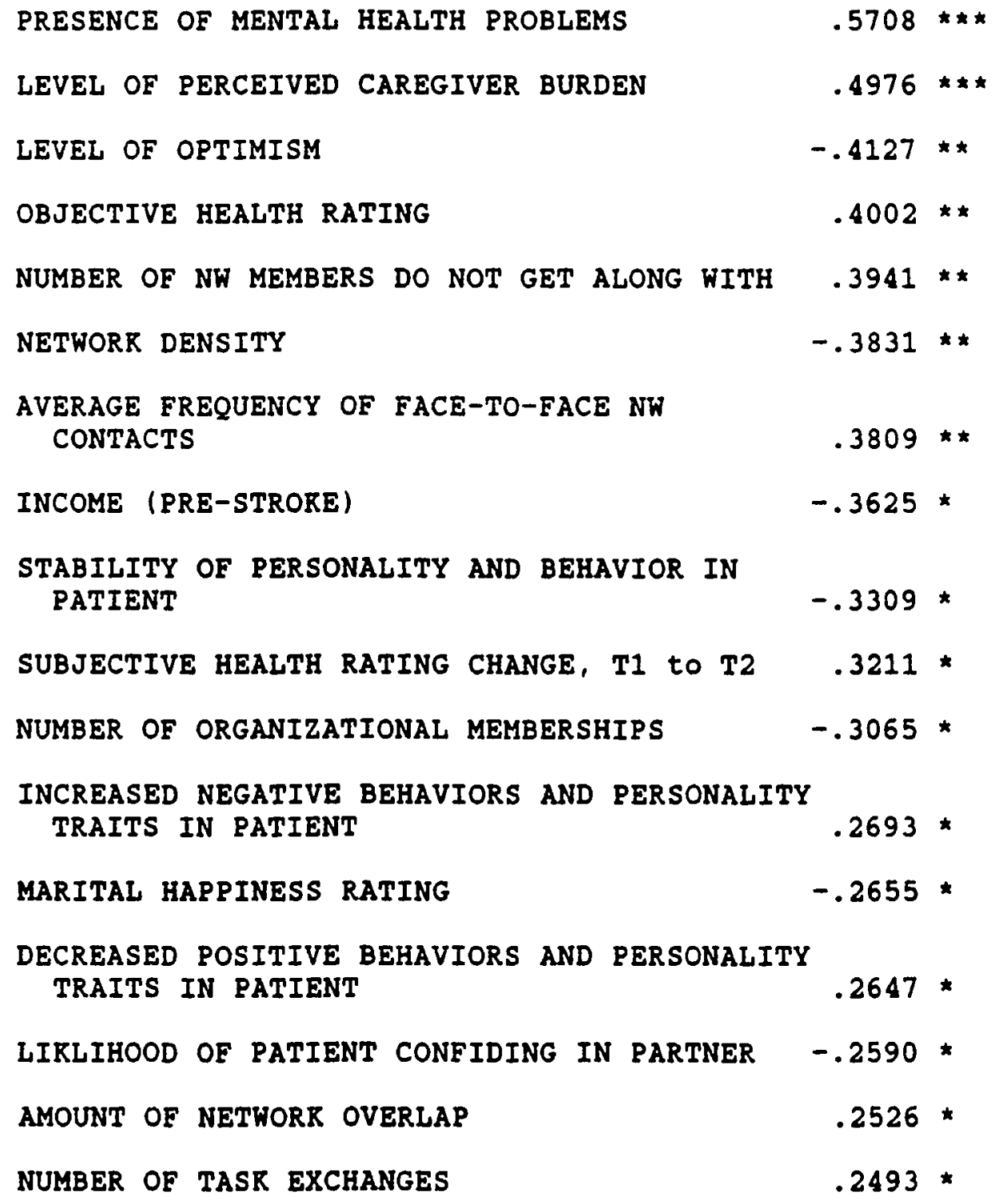


TABLE LVII

CORRELATIONS EITH CES-D FOR RATIENTS, TIME 2

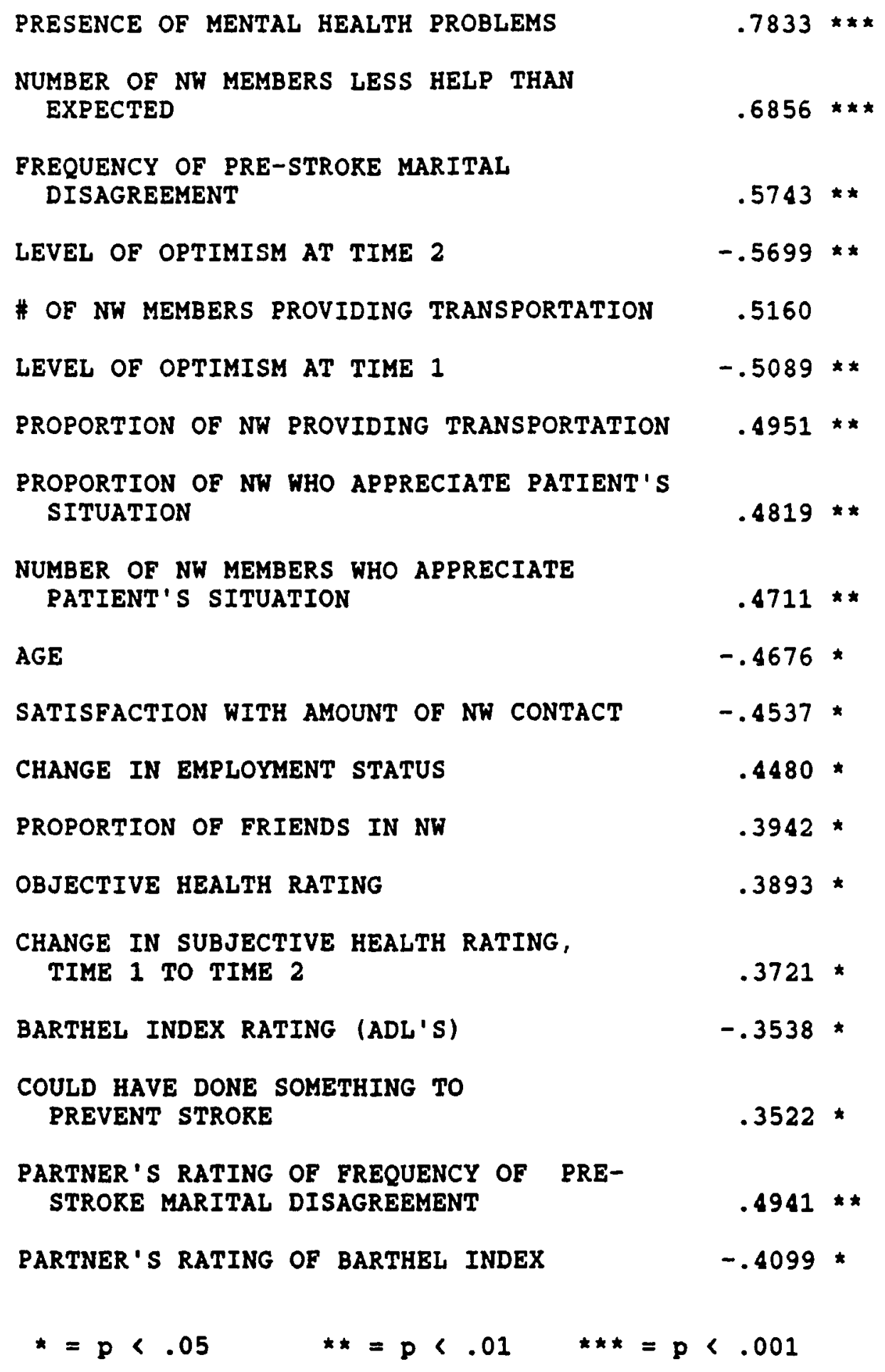


the stroke had retired or were otherwise unemployed at Time 2. Likewise, there was a decrease in the number of patients who were employed even part-time at Time 2. Clearly, the employment changes measured at Time 2 were negative ones for many patients, and it is not surprising to find a positive relationship between employment change and depression for this sample of patients. Having less income was related to higher depression scores for the caregivers Time 2 .

Level of optimism maintained its strong negative relationship to depression at Time 2 for caregivers and for patients. In addition, the patients' level of optimism at Time 1 had a strong relationship to Time 2 depression level. Health Factors. Health factors continued to be important correlates of depression at Time 2. For the caregivers, the presence of a mental health problem, and objective health rating correlated strongly and positively with depression. Patients who had sought help for a mental health problem, had higher objective health problem ratings, and who had a negative change in subjective health rating at Time 2 were more likely to be depressed. Caregivers were also more likely to have high depression scores if they reported a poorer subjective health rating at Time 2 compared to Time 1 . It is not surprising that the presence of a mental health problem correlated so highly with depression, as the two items may both be measuring depression. It is also consistent with other research that 
poorer physical health status continued to correlate substantially with depression for both patients and caregivers.

Stroke-Related Variables. Level of perceived caregiver burden correlated highly $(\underline{\underline{r}}=.50, \mathrm{p}(.001)$ with the depression score. In addition, a number of patient personality and behavior change variables were significantly related to caregiver depression levels. Less reported stability of behavioral characteristics in the patient, increased negative personality and behavior traits, and decreased positive patient behaviors all correlated positively with caregiver depression score.

For the patients, Barthel Index Rating as well as the partner's Barthel Index rating of the patient correlated negatively with patients' depression scores. Patients who continued to feel that they could have done something to prevent the stroke were more likely to have higher depression scores. In sum, for the caregivers level of perceived burden, as well as the perception of negative behavior and personality changes in the patient were more related to depression level than were more direct measures of stroke severity. For the patients, ADL level and feelings of guilt over the stroke having occurred continued to be moderately correlated with depression.

Social Networks and Social Support. Several aspects of social contact and social support continued to be related 
to depression for the caregivers. Network density was negativaly correlated with depression, and the number of network members the caregiver reported not getting along with was positively correlated with depression score. Unexpectedly, the average frequency of face-to-face network contacts correlated positively and fairly strongly with depression score for the caregivers $(\underline{\underline{x}}=.38, \mathrm{p}<.01)$. In addition, the number of task exchanges reported was positively related to depressive symptoms. A higher number of organizational memberships was negatively related to depression, while a greater amount of overlap between the caregiver's and patient's networks was positively related to depression score. These relationships between social network and support factors and depressive symptom level for the caregivers are interesting and complex. Greater network density may provide some buffering against depression in the circumstance of chronic illness, perhaps because more support is mobilized when network members know one another. At the same time, high frequency of face-toface contacts together with a high level of network overlap may create strains within the network and for the caregiver. It was not uncommon at Time 2 for caregivers to name relatives when indicating persons with whom they didn't get along. The occurrence of a stroke may make it more difficult for caregivers to avoid negative network relationships. 
For the patients at Time 2 , a number of negative aspects of network support are seen in the relationship between these variables and depression scores. The number and proportion of network members providing transportation, as well as the number and proportion of network members who reportedly appreciate the patient's situation were all positively related to depression level. This was also true regarding the proportion of friends in the network. The number of network members who were perceived to help less than expected was highly positively related to patient depression score $(\underline{x}=.69, p<.001)$. It may be that depressed patients see their network in less positive terms. It may also be the case that greater need for assistance with transportation and other types of instrumental support is indicative of stroke severity level, which is also related to depression score. Nevertheless, it is equally plausible that the persons around the patient may respond inappropriately to his or her needs, and that the presence of friends after one has suffered a stroke is a strain as well as a source of support. On the other hand, patients who reported high levels of satisfaction with the amount of network contact they had were more likely to have lower depression scores.

Marital support. A number of aspects of the partner relationship continued to be important correlates of depression. For the caregivers, a higher overall narital 
happiness rating and increased liklihood of the partner confiding in them were negatively associated with depression. For the patients, greater frequency of prestroke marital disagreement as perceived by both the patient and the caregiver was related to higher depression levels at Time 2 .

\section{Correlates of Positive Well-Being}

Table LVIII summarizes the correlations between positive well-being and other variables at Time 2 . For the caregivers, a higher level of optimism, greater frequency of social contacts, higher subjective health rating, and more positive anticipated future health change were all associated with higher levels of positive well-being. Also, a number of aspects of social support and marital support were correlated with PSWB score. Higher marital happiness rating, a greater likelihood of the partner confiding in the caregiver, more confiding exchanges, and higher satisfaction with the quality of network contacts were all related to higher levels of positive well-being for the caregivers.

For the patients, few correlations of other variables with positive well-being were significant. Frequency of shared marital activity since the stroke was highly and positively correlated with patient well-being $(\underline{x}=.53, \mathrm{p}<$ $.01)$. 
TABLE LVIII

CORRELATIOHS WITH ROSITIVE WELI-BEING, TIME 2

$\begin{array}{lr}\text { Correlates of Careqiver Positive Well-Being } \\ \text { LEVEL OF OPTIMISM } & .4630 \star \star \star \\ \text { LEVEL OF SOCIAL ACTIVITY } & .4594 \star \star \star \\ \text { RATING OF MARITAL HAPPINESS } & .3291 \star \\ \text { LIRLIHOOD OF PATIENT CONFIDING IN PARTNER } & .3234 \star \\ \text { SUBJECTIVE HEALTH RATING } & .2914 \star \\ \text { NUMBER OF CONFIDING EXXCHANGES } & .2779 \star \\ \text { SATISFACTION WITH AMOUNT OF SOCIAL CONTACT } & .2718 \star \\ \text { ANTICIPATED FUTURE HEALTH CHANGE } & -.2699 \star \\ \text { LEVEL OF OPTIMISM AT TIME } 1 & .2588 \star \\ \text { SATISFACTION WITH QUALITY OF NW CONTACT } & .2468 \star\end{array}$

Correlates of Patients' Positive Well-Being $(N=24)$

FREQUENCY OF SHARED MARITAL ACTIVITY .5250 **

EDUCATION $\quad .4347$ *

NUMBER OF ORGANIZATIONAL MEMBERSHIPS .4024 *

COULD HAVE DONE SOMETHING TO PREVENT

THE STRORE $\quad .3437$ *

$\begin{aligned} \star & =p<.05 \\ \star * & =p<.01 \\ \star \star * & =p<.001\end{aligned}$ 
Correlates of Negative Well-Being

Tables IIX and IX summarize the correlations with negative well-being at Time 2 for the major caregiver and patient variables. stroke-related factors and social network/social support factors correlated more strongly with caregiver negative well-being than did demographic or individual characteristic factors. Aspects of the partner relationship continued to be important as well.

Health Factors. For patients, subjective health rating and the number of chronic health problems correlated moderately with negative well-being. Perceived better health and fewer chronic health problems were associated with lower negative well-being. A perceived negative change in overall health status was strongly related to higher negative well-being scores $(\underline{\underline{r}}=.63, \mathrm{p}<.001)$. The only health variable which correlated significantly with negative well-being at Time 2 for the caregivers, as it did with depression score, was the presence or acknowledgement of a mental health problem, an expected positive relationship.

Stroke-Related Factors. Perceived caregiver burden was strongly related to caregiver negative well-being $(\underline{x}=$ .43, $\mathrm{p}<.001)$, as it was to depression score. Higher stroke severity rating, greater concern about another stroke, and noting more behavioral and personality changes in the patient after the stroke were all related moderately to higher negative well-being scores for the partners. The 
TABLE LIX

CORREIATIONS WITH NEGATIVE WELL-BEING FOR CAREGIVERS, TIME 2

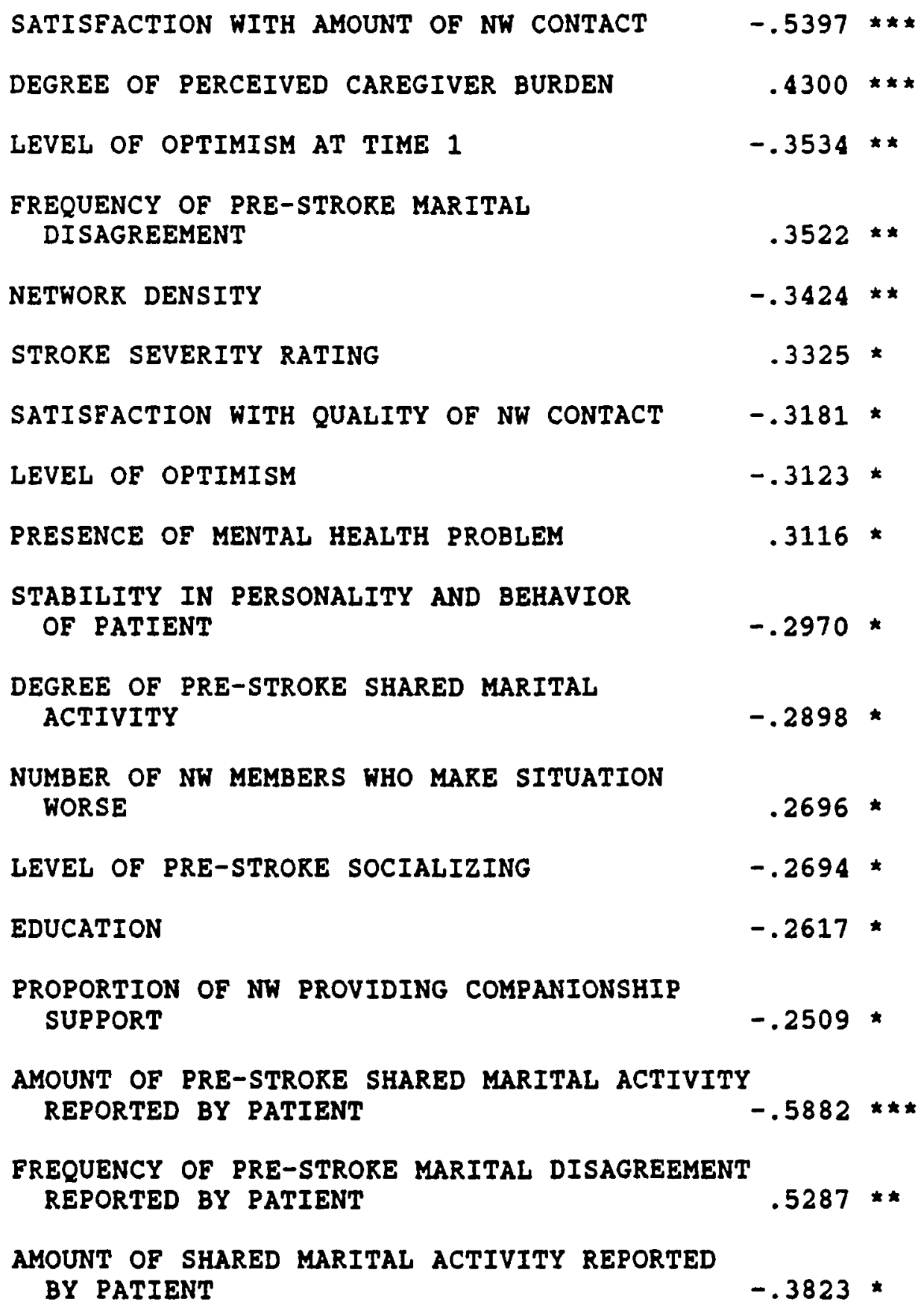

FREQUENCY OF PRE-STRORE MARITAL DISAGREEMENT REPORTED BY PATIENT

AMOUNT OF SHARED MARITAL ACTIVITY REPORTED BY PATIENT 
TABLE LX

CORRELATIONS WITH NEGATIVE WELL-BEING FOR PATIENTS, TIME $2(N=24)$

\begin{tabular}{|c|c|c|}
\hline $\begin{array}{l}\text { CHANGE IN HEALTH STATUS, TIME } 1 \text { TO } \\
\text { TIME } 2\end{array}$ & .6277 & $\star \star \star \star$ \\
\hline LEVEL OF OPTIMISM AT TIME 1 & -.5243 & $\star \star \star$ \\
\hline $\begin{array}{l}\text { NUMBER OF NW MEMBERS WHO PROVIDE TRANS- } \\
\text { PORTATION }\end{array}$ & .5139 & $\star \star$ \\
\hline SHARED OR DELEGATED CONTROL OF FINANCES & .4958 & $\star \star$ \\
\hline NETWORR DENSITY & -.4769 & $\star \star$ \\
\hline SATISFACTION WITH AMOUNT OF NW CONTACT & -.4729 & $\star \star *$ \\
\hline NUMBER OF CHRONIC HEALTH PROBLEMS & .4593 & * \\
\hline CONCERN ABOUT FUTURE CARE & .4590 & $\star$ \\
\hline $\begin{array}{l}\text { PROPORTION OF NW WHO PROVIDE } \\
\text { TRANSPORTATION }\end{array}$ & .4398 & $\star$ \\
\hline AGE & -.4340 & $\star$ \\
\hline LEVEL OF OPTIMISM & -.4325 & * \\
\hline SUBJECTIVE HEALTH RATING & -.4310 & $\star$ \\
\hline NETWORR DEGREE (OF INTERCONNECTEDNESS) &.- .213 & * \\
\hline LEVEL OF SOCIAL ACTIVITY & .3886 & * \\
\hline PROPORTION OF FRIENDS IN NH & .3810 & * \\
\hline $\begin{array}{l}\text { NUMBER OF NW MEMBERS LESS HELP THAN } \\
\text { EXPECTED }\end{array}$ & .3721 & * \\
\hline FREQUENCY OE DISAGREEMENT OVER FINANCES & .3715 & $\star$ \\
\hline INCOME & .3374 & \\
\hline $\begin{aligned} \star & =p<.05 \\
\star * & =p<.01 \\
* \star * & =p<.001\end{aligned}$ & & \\
\hline
\end{tabular}


only stroke-related factor which correlated significantly with negative well-being at Time 2 for the patients was concern about future care. Greater concern in this area was related to higher NGWB scores $(\underline{r}=.46, \mathrm{p}<.05)$.

Social Networks and Social Support. Many of the same network variables and aspects of social support which correlated with depression also showed a similar relationship to negative well-being. This is not a surprising finding, given the strong relationship between the two outcome measures. This was true of several of the social support measures for the patients, including the number and proportion of network members who provided transportation support and the number and proportion of network members who were perceived as appreciating what the patient was going through. There was also a significant positive relationship between the amount of post-stroke social activity and patient negative well-being score. Thus again with the negative well-being measures, we see evidence of both positive and negative effects of social network interaction for the patients. These findings are congruent with Hyman's (1971) notion of the "stigna" of stroke.

In the case of the caregivers, satisfaction with the amount of social network contact was associated with lower negative well-being scores $(\underline{x}=-.54, p<.001)$. Satisfaction with the quality of network interactions was similarly related to negative well-being, but not as 
strongly $(\underline{r}=-.32, p<.05)$. Congruent with these findings, a greater proportion of socializing support available within the close network was related to lower levels of negative well-being. Indications of possible strain within the networks of the caregivers was seen in the positive correlations between transportation support, and frequency of contact and negative well-being score. Perhaps satisfaction with the amount and quality of support, and socialization support imply more choice of interaction with network members, while instrumental assistance and frequency of contact imply at least some elements of constraint in choice of network association (Fisher et al., 1977).

Marital Support. For the caregivers at Time 2, various aspects of the quality of the partner interaction had stronger relationships to negative well-being than was true at Time 1. Perceiving the relationship more positively compared to other couples, and both caregiver and patient reporting more shared marital activity were associated with lower negative well-being scores. In contrast, reporting more pre-stroke disagreement within the relationship was related to higher negative well-being for the caregivers. Patients who reported higher levels of disagreement over finances and who were less involved in making financial decisions since the stroke reported higher levels of negative well-being.

Intercorrelations Among Outcome Variables at Time 2 
Table LXI presents the intercorrelations among the outcome variables at Time 2. For the most part, outcoma measures for the caregivers and patients were much more strongly related at Time 2 . This suggests that over the six months between interviews, subjects' depressive symptom levels and feelings of well-being were either influencing those feeling and mood states in the partner, that both members of the dyad were experiencing similar positive and negative influences on their well-being related to the stroke experience, or that both factors were operating. This was true for depression score, as well as positive, negative, and overall well-being levels. The one exception to this pattern was that levels of optimism for caregiver and patient became less strongly correlated at Time 2 . This finding has implications for intervention strategies which will be discussed in Chapter VIII.

\section{REGRESSION ANALYSES: TIME 2}

Hierarchical regression analyses were carried out for the major outcome variables of depression, positive wellbeing, and negative well-being at Time 2, following procedures employed at Time 1 . The model proposed in Chapter II as well as the strength of a particular variable's relationship to the outcome measure under consideration guided the choice of Time 2 predictor variables as had been the case at Time 1 . Regression 
TABLE LXI

INTERCORRELATIONS AMONG OUTCOME VARIABLES AT TIME 2

\begin{tabular}{|c|c|c|c|c|c|c|c|c|c|c|}
\hline YARIABLE & CES-D & $\underset{\text { MB }}{\text { POSITIVE }}$ & $\underset{\text { MB }}{\text { MEGATIVE }}$ & $\underset{\text { BB }}{\text { BALANCE }}$ & LOT & $\begin{array}{l}\text { PATIENTS' } \\
\text { CES-D }\end{array}$ & $\begin{array}{l}\text { PATIENTS' } \\
\text { POSITIVE } \\
\text { HB }\end{array}$ & $\begin{array}{l}\text { PATIENTS' } \\
\text { MEGATIVE } \\
\text { WB }\end{array}$ & $\begin{array}{l}\text { PATIENTS' } \\
\text { BALANCE } \\
\text { WB }\end{array}$ & $\begin{array}{l}\text { PATIENTS' } \\
\text { LOT } \\
\text { SCORE }\end{array}$ \\
\hline CES-D & 1.00 & & & & & & & & & \\
\hline $\begin{array}{l}\text { POSITIVE } \\
\text { WEL BEING }\end{array}$ & $-.3129 \star$ & 1.00 & & & & & & & & \\
\hline $\begin{array}{l}\text { MEGATIVE } \\
\text { HELL BEING }\end{array}$ & $.4819 \star \star$ & -.1697 & 1.00 & & & & & & & \\
\hline $\begin{array}{l}\text { BALAIXE } \\
\text { WELL BEING }\end{array}$ & $.5384 \star \star$ & $-.7482 t t t$ & $.7051 \star \star \star$ & 1.00 & & & & & & \\
\hline LOT SCORE & $-.4127 \star$ & $.4630 \star \star \star$ & $-.3123^{\star}$ & $-.4645 \star \star$ & 1.00 & & & & & \\
\hline PATIENTS' CES-D & $.3682 \star$ & .0325 & $.5396 \star$ & .2856 & -.0912 & 1.00 & & & & \\
\hline $\begin{array}{l}\text { PATIENTS' } \\
\text { POSITIVE MB }\end{array}$ & $-.4037 \star$ & .3145 & -.2833 & $-.4627 \star$ & -.1280 & $-.219\}$ & 1.00 & & & \\
\hline $\begin{array}{l}\text { PATIENTS' } \\
\text { MEGATIVE WB }\end{array}$ & $.5096 \star \star$ & $-.4088 \star$ & $.4182 \star$ & $.5130 \star \star$ & -.3279 & $.5404 \star \star t$ & -.1717 & 1.00 & & \\
\hline $\begin{array}{l}\text { PATIENTS' } \\
\text { BALANCE WB }\end{array}$ & $.4582 \star$ & $-.5582 \star t$ & .2719 & $.6084 \star \star \star$ & -.2817 & $.3928 \star$ & $-.5393^{\star \star t}$ & $.7265 \star$ & 1.00 & \\
\hline $\begin{array}{l}\text { PATI ENTS' } \\
\text { LOT SCORE }\end{array}$ & -.2457 & -.0157 & -.3376 & -.1685 & .1973 & $-.5699 \star \star$ & .2884 & $-.4325 \star$ & $-.4250 \star$ & 1.00 \\
\hline
\end{tabular}


analyses were again carried out with all caregivers included and with only iemaie caregivers included.

Combined Effect of Selected Factors on Time 2 Depression

Predictor variables on which depression score was regressed at Time 2 , and their order of entry into the regression equation were: objective health rating, perceived caregiver burden, marital happiness rating, density of the social network, and the proportion of the network providing instrumental help. Intercorrelations among these variables were modest, the highest being that between caregiver burden and marital happiness rating l $\underline{r}=-$ .42). Network density was negatively correlated with perceived burden $(\underline{\underline{r}}=.32)$, and positively related to marital happiness rating $(\underline{\underline{r}}=.30)$. Table LXII summarizes results of the regression analysis for Time 2 CES-D score when all caregivers were included. Slightly more of the variance in depression score was accounted for compared to Time 1 results, with $\underline{R}=.70$ and $\underline{R}=.49$. Objective health rating accounted for $16.2 \%$ of the vartance in depression score, while perceived level of caregiver burden accounted for $28.3 \%$ of the variance in depression, even after controlling for objective health factors. Network density accounted for an additional $4.3 \%$ of the variability in in depression score, while marital happiness rating and proportion of network instrumental help each contributed less than 18 to explaining level of depresssive symptoms. 
TABLE IXII

HIERARCHICAL REGRESSION FOR CES-D,

TIME 2: ALL CAREGIVERS INCLUDED

Dependent Variable CES-D

\begin{tabular}{lccccc} 
& MULTIPLE R & $\mathrm{R}^{2}$ & $\mathrm{R}^{2}$ CHANGE & SIMPLE R & BETA \\
\cline { 2 - 6 } Objective Health Rating & .403 & .162 & .162 & .403 & 0.446 \\
Perceived Caregiver Burden & .667 & .445 & .283 & .502 & 0.454 \\
Marital Happiness Rating & .670 & .449 & .004 & -.281 & -0.020 \\
Density of Network & .702 & .493 & .043 & -.360 & -0.231 \\
$\begin{array}{l}\text { Proportion of NW Providing } \\
\text { Instrumental Help }\end{array}$ & .703 & .494 & .001 & -.081 & 0.030
\end{tabular}


Table LXIII presents the results of the regression analysis when only female caregivers were considered. Although almost exactly the same amount of variance in depression was explained $\left(\underline{R}=.71, \underline{R}^{2}=.50\right)$, objective health rating now explained $21.3 \%$ and caregiver burden $23 \%$ of the variance in depression scores. Proportion of instrumental support exchanges dropped out of the regression equation when male caregivers were excluded.

Combined Effect of Selected Factors on Time 2 Positive Wel1Being

Variables which were included as predictors of positive well-being at Time 2 included subjective health rating, stroke severity rating, overall marital happiness, satisfaction with amount of social contact, and number of confiding exchanges. There was not a high degree of intercorrelation between any of variables. stroke severity rating was negatively correlated with satisfaction with amount of network contact $(\underline{\underline{x}}=.36)$ and positively with marital happiness rating $(\underline{\underline{x}}=.35)$. These were the strongest intercorrelations between any pairs of predictor variables.

Table LXIV presents the results of the hierarchical regression for positive well-being. The regression equation resulted in a Multiple $\underline{R}$ of .54 , accounting for $29 \%$ of the variance in PSWB score. At Time 2, considerably less of the variability in positive well-being score was explained by 
TABLE IXIII

HIERARCHICAL REGRESSION FOR CES-D, TIME 2: FEMALE CAREGIVERS ONLY

Dependent Varlable CES-D

ObjectIve Health Rating

MULTIPLE R

$\mathbf{R}^{2}$

$\mathrm{R}^{2}$ CHANGE

SIMPLE $R$

BETA

$\begin{array}{lcccc}.462 & .213 & .213 & .462 & 0.476 \\ .666 & .443 & .230 & .571 & 0.383\end{array}$

Percelved Careglver Burden

.666

.669

.443

.571

0.383

Marital Happiness Rating

.448

.005

$-.276$

$-0.356$

Density of Network

.706

.498

.050

$-.348$

$-0.242$ 
TABLE LXIV

HIERARCHICAL REGRESSION FOR POSITIVE

WELL-BEING, TIME 2: ALL CAREGIVERS INCLUDED

\begin{tabular}{|c|c|c|c|c|c|}
\hline \multirow[t]{2}{*}{ Dependent Variable Positive } & \multirow[b]{2}{*}{ Multiple R } & \multirow[b]{2}{*}{$\mathrm{R}^{2}$} & \multirow[b]{2}{*}{ Change } & \multirow[b]{2}{*}{ S1mple R } & \multirow[b]{2}{*}{ Beta } \\
\hline & & & & & \\
\hline SubjectIve Health Rating & .285 & .081 & .081 & .285 & 0.204 \\
\hline Stroke Severity & .330 & .109 & .028 & -.203 & -0.041 \\
\hline Marital Happiness Rating & .434 & .189 & .080 & .329 & 0.306 \\
\hline $\begin{array}{l}\text { Satisfaction with Amount } \\
\text { of Soctal Contact }\end{array}$ & .443 & .196 & .007 & .247 & 0.038 \\
\hline Number of Confiding Exchanges & .538 & .290 & .094 & .345 & 0.329 \\
\hline
\end{tabular}


the selected predictor variables than had been the case at Time 1. Even after subjective health factors and stroke severity were considered, the number of confiding exchanges accounted for the greatest amount of explained variance in positive well-being, 9.4\%. Subjective health rating and marital happiness score each accounted for about $8 \%$ of the variability in positive well-being, and stroke severity accounted for 2.8\%. Satisfaction with amount of social contact accounted for less than $1 \%$ of the variance in positive well-being. These results are consistent with other studies which have demonstrated the importance of confiding relationships to well-being lGoldberg et al., 1985; Lowenthal \& Haven, 1968).

Slightly less of the variability in PSWB score was explained by the regression equation which resulted when male caregivers were excluded. This information is contained in Table LXV. A regression equation with $\underline{R}=.52$ accounted for $26.6 \%$ of positive well-being variance. Marital happiness rating became the strongest predictor of positive well-being for female caregivers, explaining $10.5 \%$ of the variance after controlling for subjective health and stroke severity. Number of confiding exchanges accounted for $6.4 \%$ of the variance in positive well-being, and stroke severity rating explained less than $1 \%$ for the female caregivers. 
TABLE IXV

HIERARCHICAL REGRESSION FOR POSITIVE

WELL-BEING, TIME 2: FEMALE CAREGIVERS ONLY

Dependent Variable Positive

Well-Being

\begin{tabular}{|c|c|c|c|c|c|}
\hline & MULTIPLE R & $\mathrm{R}^{2}$ & $\mathrm{R}^{2}$ CHANGE & SIMPLE R & BETA \\
\hline Subjective Health Rating & .292 & .085 & .085 & .292 & 0.228 \\
\hline Stroke Severity Rating & .301 & .091 & .005 & -.114 & 0.034 \\
\hline Marital Happlness Rating & .442 & .195 & .105 & .370 & 0.338 \\
\hline $\begin{array}{l}\text { Satisfaction with Amount of } \\
\text { Soclal NW Contact }\end{array}$ & .449 & .201 & .006 & .193 & 0.023 \\
\hline Number of Confiding Exchanges & .516 & .266 & .064 & .275 & 0.269 \\
\hline
\end{tabular}


Combined Effects of Selected Factors on Time 2 Negative Hell-Being
Negative well-being was regressed on stroke severity, perceived caregiver burden, amount of shared marital activity, satisfaction with amount of network contact, and network density at Time 2, with the variables entered into the regression in that order. Highest intercorrelation between any of the variables was the negative relationship between perceived burden and satisfaction with amount of network contact $(\underline{r}=-.56)$. Relationship between stroke severity rating and satisfaction with amount of network contact was moderate $(\underline{\underline{x}}=-.43)$, while the correlation between network density and perceived burden was $\underline{\underline{r}}=-.36$.

Table LxVI presents the results of the hierarchical regression for negative well-being at Time 2 for all caregivers. The regression equation had a Multiple $\underline{R}$ of .591 and an $\underline{\underline{R}}$ of .350. Stroke severity accounted for $13.2 \%$ of the variance in negative well-being, and perceived burden 7.6\% of the variability in NGWB score. After controlling for stroke severity and degree of perceived burden, satisfaction with amount of network contact explained an additional $11.3 \%$ of negative well-being variance and network density about $3 \%$. Level of shared marital activity explained less than $1 \%$ of the variability in negative wellbeing level.

When female caregivers only mere included in the 
TABLE LXVI

HIERARCHICAL REGRESSION FOR NEGATIVE

WELL-BEING, TIME 2: ALL CAREGIVERS INCLUDED

Dependent Variable $\begin{array}{r}\text { Negative } \\ \text { Well-Belng }\end{array}$

\begin{tabular}{|c|c|c|c|c|c|}
\hline & MULTIPLE $R$ & $\mathrm{R}^{2}$ & $\mathrm{R}^{2}$ CHANGE & SIMPLE $\mathrm{R}$ & BETA \\
\hline Stroke Severity & .363 & .132 & .132 & .363 & 0.092 \\
\hline Percelved Caregiver Burden & .456 & .208 & .076 & .431 & 0.089 \\
\hline Amount of Shared Marital Activity & .456 & .208 & .000 & -.055 & -0.020 \\
\hline $\begin{array}{l}\text { Satisfaction with Amount of NW } \\
\text { Contact }\end{array}$ & .567 & .321 & .113 & -.538 & -0.397 \\
\hline Network Density & .591 & .350 & .029 & -.342 & -0.185 \\
\hline
\end{tabular}


regression analysis, the amount of variance in negative weil-being which was accounted for increased to $39 \%$. This information is summarized in Table LXVII. For the female caregiver sample, stroke severity predicted 298 of the variability in negative well-being, or $74 \%$ of the explained variability. The contribution of perceived burden decreased to $2.9 \%$ from 7.6\%. Satisfaction with amount of network contact explained $5.7 \%$ of the variance in negative wellbeing for the female caregivers, while the contributions of network density and the level of shared marital activity remained small.

SUMMARY: CORRELATES AND PREDICTORS OF WELL-BEING, TIME 1 AND TIME 2

At both Time 1 and Time 2, health factors, strokerelated variables, and a number of aspects of network and marital support were related to depression and well-being. The direction of relationship vetween instrumental support measures and the outcome variables and between some interactional measures of network contact and well-being provided evidence of the complex and multidimensional nature of support. Both negative and positive effects of perceived support were seen for patients as well as caregivers.

When all partners were included, the strongest predictor of depression in the caregiver group at Time 1 was pre-stroke subjective health rating. At Time 2, the greatest amount of variance in depression was explained by 
TABLE LXVII

HIERARCHICAL REGRESSION FOR NEGATIVE

WELL-BEING, TIME 2: FEMALE CAREGIVERS ONLY

Dependent Variable Negative

We11-Being

\begin{tabular}{lccccc} 
& MULTIPLE $\mathrm{R}$ & $\mathrm{R}^{2}$ & $\mathrm{R}^{2}$ CHANGE & SIMPLE R & BETA \\
\cline { 2 - 6 } Stroke Severity & .538 & .290 & .290 & .538 & 0.265 \\
Percelved Caregiver Burden & .565 & .319 & .029 & .457 & 0.037 \\
Amount of Shared Marital Activity & .566 & .320 & .001 & -.104 & -0.068 \\
$\begin{array}{l}\text { Satisfaction with Amount of NW } \\
\quad \text { Contact }\end{array}$ & .614 & .378 & .057 & -.548 & -0.335 \\
Density of Network & .625 & .390 & .013 & -.316 & -0.127
\end{tabular}


level of perceived caregiver burden. For positive wellbeing at Time 1 , the strongest predictor was the patient's level of functional ADL independence, while at Time 2 it was subjective health rating and marital happiness rating. Negative well-being score was best predicted by subjective health rating at Time 1 and by stroke severity rating at Time 2. Some differences were observed both in the amount of variance accounted for and in the relative explanatory power of the predictor variables when only female caregivers were considered in the hierarchical regression analyses, but these differences in general were small.

Chapter VII will explore the distribution of change in depression and well-being scores over time, and predictors of change in these outcome measures over time. 
CHAPTER VII

CHANGES IN DEPRESSION AND WELL-BEING OVER TIME

This chapter will describe the distribution of change in depression and well-being scores over time for patients and caregivers, as well as changes in other variables over time. Finally, results of regression analyses of predictors of change over time which were carried out for the caregiver outcome variables will be reported.

\section{DESCRIPTIVB AND CORRELATIONAL ANALYSIS \\ OF CHANGE OVER TIME}

\section{Change in Depression Scores Over Time - CES-D}

For the caregivers, average change score on the CES-D between Time 1 and Time 2 was 1.73 (S. D. $=12.61$ ), the positive number indicating an overall decrease in depression score over time. For the most part, depression scores remained quite stable, although individual change scores fluctuated as much as 31 points in either direction (range = -30 to +31 ). Three caregivers experienced a decrease in depression score which was more than one $S$. D. from the mean, and 7 caregivers (16\%) had an increase in depression score which was more than one $S$. D. from the mean change score. The rest of the partners had change scores which fluctuated between -10 (reflecting an increase in depression score at Time 2 ) and +11. Seventy-five percent of the caregivers had Time 2 depression scores which were within 
ten points of their Time 1 score, and $50 \%$ had depression change scores of six points or less. Correlation between Time 1 and Time 2 depression scores for the caregivers was $\underline{r}$ $=.55, p<.001$.

Patients had a mean depression change score of -1.77 (S. D. = 13.05), indicating an overall increase in depression score over time. Range of change scores for the patients was from -35 (indicating an increase in depression score) to +14 . Forty-five percent of the patients for whom depression scores were available for both interview times had an increase in depressive symptoms over time, and four experienced an increase which was more than one $S$. D. from the mean change score. Fifty percent of the patients reported a decrease in depression score, but only two patients had depression score decreases which were more than one S. D. from the mean. Fifty-five percent of the patients had Time 2 depression scores which were within ten points of their Time 1 depression score, while $33 \%$ had a CES-D change score of six points or less. Correlation between the Time 1 and Time 2 depression scores for the patients was $\underline{\underline{r}}=.56$, $p$ $<.01$.

Thus, for the subjects in this sample, there was a scattered distribution of change scores for the CES-D, with very few respondents experiencing changes in depressive symptoms which were more than one S. D. in either direction from the mean change score. Slightly less than $20 \%$ of the 
patients and 168 of the partners had significant increases in depression score over time.

\section{Change in Positive Well-Being Over Time}

The mean change in caregiver positive well-being score over time was very small, .04 (S. D. = 2.30). Range of change scores was from -6 to +5 . Fourteen caregivers (30\%) had no change in positive well-being score over time, while about one-third experienced an increase and one-third had a decrease in PSWB score. Four partners had a positive change score (higher PSWB score at Time 2) which was more than one S. D. Erom the mean, and six (13\%) had a negative change score (lower PSWB score at Time 2) which was more than one S. D. from the mean change score. The correlation between Time 1 and Time 2 PSWB scores for the caregivers was $\underline{r}=$ $.63, p<.001$.

Patients had a moderate degree of change in positive mell-being scores over time, mean $=.74, \mathrm{~S} . \mathrm{D} .=2.86$. This reflected an overall decrease in positive well-being over time. Range of change scores was -4 to +8 . Eight patients experienced an increase in PSWB score, with the increase for three subjects being more than one $S$. D. from the mean change score. Thirteen patients had a decrease in PSWB score over time, six having a decrease which was more than one S. D. from the mean change score. Thus about 25\% of the respondent patients had a significant decrease in reported positive well-being at Time 2 . For the patients, 
correlation between Time 1 and Time 2 PSWB scores was minimal, $\underline{r}=.02$. This would suggest that those patients who felt more positive at Time 1 were not necessarily the same individuals who viewed their situation in relatively positive terms at Time 2 .

\section{Change in Negative Well-Being Over Time}

Caregivers were about equally divided among those who showed an increase in negative well-being, those who showed no change, and those who had a decrease in negative wellbeing over time. The mean change score for negative wellbeing was .26, S. D. $=2.17$. Change scores ranged from -4 to +6. Five caregivers (11\%) showed an increase in negative well-being which was more than one $S . D$. from the mean, and six (13\%) had a decrease in NGWB score which was more than one S. D. from the mean. The correlation between NGWB scores at Time 1 and Time 2 for the caregivers was $.69, \mathrm{p}$ < .001 .

The patients' pattern of change scores on the negative well-being measure was similar to that of the caregivers, although the mean change score was in the opposite direction. Mean change score was -.30 (S. D. $=2.38$ ), indicating an overall increase in reported negative wellbeing over time. Change scores for the patients ranged from -5 to +5 . Of the elght patients who showed an increase in negative well-being over time, five (22\%) had change scores which were more than one $S$. D. from the mean change score. 
Of the seven patients who showed a decrease in NGWB score over time, three (13x) had change scores which were more than one S. D. from the mean. The correlation between patients' Time 1 and Time 2 NGWB scores was $\underline{x}=.69$, with $p<.001$.

In summary, changes in IPWB scores for the patients were in the expected direction of lower positive well-being and higher negative well-being and balance well-being scores at Time 2, but for most of the patients these changes were not significant. The caregivers as a group had stable positive and balance well-being scores, and slightly improved negative well-being scores.

Change in Optimism Over Time - LOT

The notion that optimism is a stable personality trait was tested for this sample by examining the distribution of change scores over time for the Life Orientation Test (LOT). Caregivers had a mean change score on the LOT of 1.39, S. D. $=4.40$, with the change scores ranging from -8 (indicating an increase in optimism score at Time 2) to +13. Only three caregivers showed no change in optimism scores. Twenty six careglvers (59\%) had change scores indicating decreased optimism, with 11 respondents (448) showing a decrease in optimism which was more than one $S$. D. from the mean. Fifteen partners had change scores which indicated an increase in optimism at Time 2, with four showing an increase which was more than one S. D. from the mean. 
Correlation between the two Lot scores for the caregivers sas $\underline{r}=.49, p<.001$. A t-test indicated a significant decline in optimism score for the caregivers at Time 2 ( $\underline{t}=$ $2.09, p(.05)$.

Patients also showed an overall decrease in optimism at Time 2. Mean change score for the LOT for the patient group was 1.13, S. D. $=5.95$, range $=-9$ to +18 . The same number of patients (10) showed an increase in optimism score as showed a decrease. Two patients showed a significant (more than one S. D. from the mean) increase and two showed a significant decrease in level of optimism at Time 2 . Correlation between the patients' Time 1 and Time 2 LOT scores was $\underline{\underline{r}}=.44, p<.05$. The overall difference in the Time 1-Time 2 mean LOT scores for the patient group was not significant.

For the caregivers in this sample, then, level of optimism did show significant negative change. This would suggest that in the context of a chronic, disabling illness, level of optimism should be viewed as a dependent, rather than an independent variable. The strains and "daily hassles" of caring for a disabled individual, and of coping with negative behavioral and personality changes in one's partner may make it very difficult to sustain an optimistic attitude.

Change in other Variables over Time

A number of $t$-test comparisons between Time 1 and Time 
2 scores were conducted on the major variables employed in this study. Significant resuits of these analyses will be discussed briefly in the following sections. All reported ttest results utilized two-tailed tests of significance. Results of these change-based t-tests should be interpreted with caution, however, because of the possibility of regression toward the mean effects and because of the increased likelihood of Type 1 errors when change-based, multiple t-tests are used.

Activity Level. For the caregivers, both overall level of religious activity and attendance at religious functions declined at Time 2 . For religious activity, the $\underline{t}$ value was $2.38, p<.05$. For attendance at religious functions, the $t$ value was $2.42, p<.05$. Caregivers' scores on the religiosity index declined at Time $2(\underline{t}=$ 3.53, $p=.001)$. Patients reported some decline overall in their level of socializing at Time 2 , but this difference did not reach significance.

Health Factors. Significant changes in self-reported health measures were found only for the patients. The patients had higher objective health problem ratings at Time $2(\underline{t}=-4.16, p<.001)$ and less optimistic expectations for their future health $(\underline{t}=-2.84, p<.01)$. Distribution of change scores on the subjective health rating measure was examined in order to determine whether there was a subgroup of caregivers who reported significant 
perceived changes in their health after the stroke. Mean change in overall haalth rating mas $.02, \mathrm{~S} . \mathrm{D} .=.92$. Twenty-four (48\%) of the caregivers had no change in their health rating score at Time 2 . Eleven (22\%) reported their health to be worse by one scale score (on a five point scale), and two reported their health to be worse by two scale scores. Ten caregivers gave themselves an overall health rating which was one scale score better at Time 2 , and three had an overall health rating which was two scale scores better at Time 2. Thus, $75 \%$ of the caregivers rated their own health as the same or somewhat better six to eight months after the stroke.

Stroke-Related Factors. The caregivers' mean rating of the patients on the Barthel Index increased significantly at Time $2(\underline{t}=-3.70, p=.001)$, a finding consistent with other studies of stroke outcome. Not unexpectedly, the group of caregivers as a whole were significantly less concerned about the possibility of another stroke at Time 2 . It should be kept in mind, however, that the partners expressed fairly high levels of concern about the possibility of another stroke at both Time 1 and Time 2 , and that their responses to open-ended questions at the end of the interview indicated that concern about another stroke was the greatest stroke-related worry or concern of a substantial number of both caregivers and patients.

Social Networks and Social Support. Caregivers 
reported significantly more people in their overall networks at Time $2(\underline{t}=-2.77, p<.01)$. They reported significantly fewer reciprocal task exchanges $(\underline{t}=2.33, p<.05)$ and $a$ decreased proportion of the close network providing instrumental help at Time $2(\underline{t}=2.86, p<.01)$.

Marital support. Few significant Time 1-Time 2 differences based on t-test comparisons were found for the various marital support measures. Patients reported a significantly increased likelihood that they would confide in their partner at Time $2(\underline{t}=-2.08, p<.05)$, while the caregiver group reported a decreased likelihood that they would confide in their partner at Time $2(\underline{t}=2.75, p<$ $.01)$. Caregivers also indicated a decreased frequency of marital disagreement at Time $2(\underline{t}=2.65, p=.011)$. Whether this is because the actual frequency of disagreement declined related to increased marital harmony or because the caregivers were more reluctant after the stroke to express opinions which were divergent from the patients' views is not known.

\section{REGRESSION ANALYSES}

\section{Change-Focused Rearession Analysis}

A number of changed-focused hierarchical regression analyses were completed as the final step in the analysis of the data generated by this study. The purpose of these regression analyses was to investigate the relative 
contributions of key variables over time to the prediction of the dependent variables, caregiver depression and wellbeing scores at rime 2 . The change-focused regression analysis approach adopted for this research was described by Cronbach and Furby (1970) and has been utilized in other longitudinal studies (Palmore \& Rivett, 1977). Variables are entered into the regression equation in a specified order, such that the effects of a particular variable at Time 1 are accounted for immediately before the effects of that variable at Time 2 are measured. Utilizing this method of assessing the predictive value of key Time 1 and Time 2 variables permits an estimate of the relative influence of residual change in a variable over time in explaining the variance in the dependent variable. Residual change is the change that results independently of the change that would be predicted on the basis of initial score on the variable (Palmore \& Rivett, 1977). The order in which variables are entered into the regression equation has the effect of controlling for the initial levels of the predictor variables when looking at the effects of changes in those variables over time. For example, if subjective health rating at Time 1 is entered into the equation first, followed by subjective health rating at Time 2 , any additional variance in the dependent variable explained by Time 2 subjective health ( $\underline{R}^{2}$ Change) is the result of change in subjective health over time. 


\section{Contributors over Time to Time 2 Depression Score}

Time 2 depression score was regressed on a number oi key predictor variables, including Time 1 depression score, subjective health rating at Time 1 and Time 2 , the patient's level of ADL functioning at both points in time, and the proportion of reciprocal confiding relationships before and six monsths after the stroke. Results of the regression analysis when all caregivers were included are presented in Table LXVIII. The regression equation had a multiple $\underline{R}$ of .78 and an $R$ of .64. Depression score at Time 1 was entered first into the equation, accounting for $30.5 \%$ of the variance in Time 2 depression level and almost $40 \%$ of the explained variance. Subjective health rating at Time 1 was entered next into the regression equation, followed by subjective health rating at Time 2 in order to control for perceived health factors, and to determine whether changes in perceived health over time helped to explain the variance in depression scores. These variables contributed little to the explained variance in Time 2 depression score, with each accounting for less than 18 of the remaining variance. The high degree of stability in the caregivers' health ratings over time together with the strong relationship between subjective health and Time 1 depression level probably account for the small amount of variance in Time 2 depression explained by subjective health factors. The patients' Time 1 level of ADL functioning was the next 
TABLE LXVIII

HIERARCHICAL REGRESSION FOR CES-D, TIME 1 AND TIME 2: ALL CAREGIVERS

Dependent Varlable CES-D TIme 2

CES-D, $T_{1}$

Subjective Health Rating, $T_{1}$

Subjective Health Rating, $T_{2}$

Barthel Index, $\mathrm{T}_{1}$

Barthel Index, $\mathrm{T}_{2}$

Proportion Reciprocal Confiding, $\mathrm{T}_{1}$

Proportion Reciprocal Confiding, $\mathrm{T}_{2}$

Percelved Caregiver Burden

\begin{tabular}{ccccc} 
MULTIPLE R & $\mathrm{R}^{2}$ & $\mathrm{R}^{2}$ CHANGE & SIMPLE R & BETA \\
\hline .552 & .305 & .305 & .552 & 0.499 \\
.554 & .307 & .002 & -.204 & -0.040 \\
.559 & .313 & .006 & -.185 & 0.072 \\
.596 & .355 & .043 & -.051 & 0.345 \\
.607 & .369 & .013 & -.088 & -0.039 \\
.618 & .382 & .012 & -.334 & -0.141 \\
.672 & .452 & .070 & -.341 & -0.251 \\
.784 & .614 & .162 & .496 & 0.454
\end{tabular}


variable entered, and it contributed an additional 4.38 of the explained variance in Time 2 depression score. Barthel Index score at Time 2 only accounted for an additional 1.38 of the variance in depression at Time 2. Thus, change in ADL functioning for the patient did not appear to be an important predictor of caregiver depression score at Time 2. Change in the proportion of reciprocal confiding relationships reported at Time 2 explained $7 \%$ of the remaining variance in Time 2 depression level, after controlling for the proportion of reciprocal confiding relationships in the pre-stroke network. Entered last into the regression equation was perceived caregiver burden, a Time 2 measure. This variable proved to be a strong predictor of caregiver depression, accounting for an additional $16.2 \%$ of the explained variance in Time 2 depression level.

In summary, the depression score at Time 1 accounted for almost $40 \%$ of the explained variance in Time 2 depression level for the caregivers. Even controlling for a number of other factors such as perceived health status and the patient's level of ADL independence, the degree of perceived caregiver burden was a powerful predictor of caregiver depression scores at Time 2. Change in the proportion of reciprocal confiding relationships at Time 2 accounted for $7 \%$ of the variance in depression score at Time 2. This is congruent with results obtained by Goldberg and 
her colleagues (1985), who found high levels of depressive symptomatology in older women with low levels of intimacy within their networks.

Results of the regression analysis when only female caregivers were considered were similar. This information is included in Table LXIX. Excluding the male caregivers resulted in a regression equation with $\underline{R}=.80$, accounting for 63.7\% of the variance in Time 2 depression score. Depression score at Time 1 accounted for slightly less of the explained variance in Time 2 depression score, $32.3 \%$. Change in subjective health rating at Time 2 explained only an additional $1 \%$ of the variability in Time 2 depression level after controlling for baseline subjective health. Again, the strong relationship between Time 1 depression level and subjective health resulted in Time 1 subjective health explaining none of the variance in Time 2 depression score for the female caregivers. The proportion of confiding exchanges in the pre-stroke network explained more of the variance (6\%) than did change in the proportion of confiding exchanges over time, which accounted for $2.9 \%$ of the variance in depression level after pre-stroke level of network intimacy was accounted for. Perceived level of caregiver burden was only slightly less important in predicting Time 2 depression score for the female caregivers, accounting for $13.2 \%$ of the explained variance in the dependent measure. 
TABLE LXIX

HIERARCHICAL REGRESSION FOR CES-D, TIME 1 AND TIME 2: FEMALE CAREGIVERS ONLY

Dependent Variable CES-D, Time 2

\begin{tabular}{ccccc} 
MULTIPLE R & $\mathrm{R}^{2}$ & $\mathrm{R}^{2}$ CHANGE & SIMPLE R & BETA \\
\hline .569 & .323 & .323 & .569 & 0.510 \\
.569 & .323 & .000 & -.253 & -0.136 \\
.578 & .334 & .010 & -.253 & 0.149 \\
.617 & .380 & .046 & -.055 & 0.401 \\
.646 & .417 & .037 & -.169 & -0.121 \\
.691 & .477 & .060 & -.509 & -0.181 \\
.711 & .506 & .029 & -.293 & -0.223 \\
.798 & .637 & .132 & .470 & 0.414
\end{tabular}


Contributors over Time to Positive We11-Being at Time 2

Hierarchical regression analyses were carried out for Time 2 positive well-being, with Time 1 positive well-being entered first into the regression equation. Other variables included in the regression were Barthel index score at Time 1, followed by Time 2 Barthel score, marital happiness rating at Time 1 , followed by Time 2 marital happiness rating, and the total number of confiding exchanges at Time 1 and Time 2. Results of the regression analysis for positive well-being are summarized in Table LXX for all caregivers and in Table LXXI for female caregivers only.

When all caregivers were included, the prediction of Time 2 positive well-being resulted in a regression equation with $\underline{R}=.74$ and $\underline{R}=.54$. PSWB score at Time 1 was the best predictor of positive well-being at Time 2, explaining $33.8 \%$ of the total variance and $62 \%$ of the variance accounted for by the regression equation. The next best predictor of positive well-being at Time 2 was the number of confiding exchanges in the pre-stroke network, accounting for $7 \%$ of the variance, followed by the patient's Barthel Index score at Time 1 as rated by the caregiver $14.3 \%$ of the variance). Change in the number of confiding exchanges over time accounted for an additional $1.1 \%$ of the variance in Time 2 positive well-being, and change in marital happiness rating at Time 2 explained $3.9 \%$ of the variability in positive well-being. Time 2 Barthel Index score explained 


\section{TABLE LXX}

HIERARCHICAL REGRESSION FOR POSITIVE WELL-BEING, TIME 1 AND TIME 2: ALL CAREGIVERS

\section{Dependent Variable Positive}

Wel1-Being, Time 2

\begin{tabular}{|c|c|c|c|c|c|}
\hline & MULTIPLE $\mathrm{R}$ & $\mathrm{R}^{2}$ & $\mathrm{R}^{2}$ CHANGE & SIMPLE R & BETA \\
\hline Positive Well-Being, $\mathrm{T}_{1}$ & .582 & .338 & .338 & .582 & 0.430 \\
\hline Barthel Index, $T_{1}$ & .617 & .381 & .043 & .167 & 0.238 \\
\hline Barthel Index, $\mathrm{T}_{2}$ & .623 & .389 & .008 & -.100 & -0.095 \\
\hline Marital Happiness Rating, $T_{1}$ & .627 & .393 & .005 & .137 & -0.204 \\
\hline Marital Happiness Rating, $T_{2}$ & .657 & .432 & .039 & .304 & 0.325 \\
\hline $\begin{array}{l}\text { Total Number of Confiding } \\
\text { Exchanges, } \mathrm{T}_{1}\end{array}$ & .709 & .502 & .070 & .406 & 0.162 \\
\hline $\begin{array}{l}\text { Total Number of Confiding } \\
\text { Exchanges, } \mathrm{T}_{2}\end{array}$ & .737 & .543 & .041 & .406 & 0.264 \\
\hline
\end{tabular}


TABLE LXXI

HIERARCHICAL REGRESSION FOR POSITIVE WELL-BEING,

TIME 1 AND TIME 2: FEMALE CAREGIVERS ONLY

Dependent Variable Positive

Well-Being, Time 2

\begin{tabular}{|c|c|c|c|c|c|}
\hline & MULTIPLE R & $\mathrm{R}^{2}$ & $\mathrm{R}^{2}$ CHANGE & SIMPLE R & BETA \\
\hline Positive We11-Belng, $\mathrm{T}_{1}$ & .517 & .267 & .267 & .517 & 0.367 \\
\hline Barthel Index, $T_{1}$ & .584 & .341 & .074 & .241 & 0.406 \\
\hline Barthel Index, $T_{2}$ & .620 & .384 & .043 & -.104 & -0.231 \\
\hline Marital Happiness Rating, $\mathrm{T}_{1}$ & .634 & .403 & .018 & .129 & -0.182 \\
\hline Marital Happiness Rating, $\mathrm{T}_{2}$ & .681 & .464 & .061 & .361 & 0.370 \\
\hline $\begin{array}{l}\text { Total Number of Confiding } \\
\text { Exchanges, } T_{1}\end{array}$ & .696 & .484 & .020 & .274 & 0.090 \\
\hline Exchanges, $\mathrm{T}_{2}$ & .715 & .511 & .027 & .311 & 0.208 \\
\hline
\end{tabular}


little of the variance in well-being at Time 2 after the patient's initial level of hDL indepandence was taken into account. Conversely, pre-stroke marital happiness accounted for less of the variability in Time 2 PSWB score than did change in the perception of marital happiness over time. Thus we see that while positive change in the assessment of marital happiness appeared to contribute to positive wellbeing in the caregivers at Time 2, both the pre-stroke number of confiding exchanges in the network and changes in the number of confiding exchanges over time were important for caregiver positive well-being.

When only female caregivers were considered, slightly less of the variability in positive well-being at Time 2 was explained, with $\underline{R}=.72$ and $\underline{R}^{2}=.51$. Time 1 PSWB score accounted for somewhat less of the variance in positive well-being at Time 2, 26.7\%. Barthel Index score at Time 1 explained $7.4 \%$ of the variance in Time 2 positive wellbeing. Interestingly, change in the patient's Barthel Index score over time accounted for an additional $4.3 \%$ of the variance in PSWB score at Time 2 , but the relationship was a negative one. Marital happiness rating also had more predictive power for the female caregivers, with change in marital happiness explaining an additional $6.1 \%$ of the variance in Time 2 PSWB score after controlling for prestroke marital happiness. While the total number of confiding exchanges continued to explain some of the 
variability in positive well-being, they were less powerful predictors for the female caregivers after the effects of other variables were controlled. The total number of confiding exchanges in the pre-stroke network explained $2 \%$ of the PSWB score variance, while change in the number of confiding relationships reported over time accounted for an additional 2.7 percent of the variance in Time 2 positive we11-being.

\section{Contributors over Time to Neqative Nell-Being at Time 2}

The hierarchical regression equation for negative well-being at Time 2 included negative well-being score at Time 1 as the first predictor variable. Subjective health rating at Time 1 and Time 2 , stroke severity rating at Time 1 and Time 2, satisfaction with the quality of network contacts at Time 1 and Time 2, perceived caregiver burden, and site of lesion as a dummy variable were the other variables included. They were entered in the order specified above. Left hemisphere site of lesion was entered before right hemisphere site of lesion.

Results of the regression analysis for all caregivers are presented in Table LXXII. The regression equation had a multiple $\underline{R}$ of .80 and an $\underline{R}$ of .64 . As expected, negative well-being score at Time 1 was the strongest predictor of Time 2 negative well-being level, accounting for $46.2 \%$ of the variance in negative well-being at Time 2 , and $72 \%$ of the total explained variance. Subjective health rating 
TABLE LXXII

HIERARCHICAL REGRESSION FOR NEGATIVE WELL-BEING, TIME 1 AND TIME 2: ALL CAREGIVERS

Dependent Varlable Negative

Well-Belng, Time 2

Negative We11-BeIng, $\mathrm{T}_{1}$

$\begin{array}{ccccc}\text { MULTIPLE R } & \mathrm{R}^{2} & \mathrm{R}^{2} \text { CHANGE } & \text { SIMPLE } \mathrm{R} & \text { BETA } \\ .680 & .462 & .462 & .680 & 0.380 \\ .680 & .462 & .000 & -.250 & -0.084 \\ .680 & .463 & .001 & -.105 & 0.251 \\ .697 & .486 & .023 & .237 & -0.035 \\ .726 & .527 & .041 & .348 & 0.094 \\ .729 & .532 & .005 & -.421 & -0.034 \\ .731 & .534 & .003 & & -.365 \\ .745 & .556 & .021 & .414 & 0.325 \\ .797 & .635 & .0040 & .379 & -0.110 \\ .799 & .639 & .004 & -0.462\end{array}$


contributed less than 18 to explaining the variance in negative well-being at Time 2 , once Time 1 NGHB score yas considered. This is not surprising, given that subjective health rating was the strongest predictor of negative wellbeing at Time 1 , and that the caregivers' subjective health ratings remained quite stable over time.

A left hemisphere site of lesion was the next best predictor of negative well-being at Time 2 , explaining $8 \%$ of the variance in caregiver NGWB score. This relationship between left hemisphere site of lesion and caregiver negative well-being was a negative one, as previously noted. In other words, patients with right hemisphere or other types of lesions were more likely to have partners with high negative well-being scores. Stroke severity at Time 2 accounted for $4.1 \%$ of the variance in caregiver negative well-being score, after controlling for stroke severity rating at Time 1 . This finding suggests that those caregivers who saw less positive change in their partners over time in terms of what the patient could do for himself or herself experienced higher levels of negative well-being over time, regardless of initial perceived severity of the stroke-related problems. Perceived caregiver burden explained an additional $2.1 \%$ of the variability in caregiver negative well-being at Time 2 , after controlling for initial negative well-being score, caregiver subjective health and perceived stroke severity. The social support measure, 
satisfaction with quality of network contacts before the stroke, explained less than $1 \%$ of the variability in negative well-being at Time 2 . Change in satisfaction with the quality of network contacts after the stroke was not a strong predictor of negative well-being.

For the female caregivers, the regression analysis resulted in a Multiple $\underline{R}$ of .84 , accounting for $71.2 \%$ of the variance. This information is summarized in Table LXXIII. Negative well-being score at Time 1 accounted for slightly less of the variance in Time 2 NGWB score for the female caregivers, 44.3\%. Stroke severity rating at Time 1 and at Time 2 independently accounted for more variability in caregiver negative well-being, $6.1 \%$ and $7 \%$ respectively. Thus we see an even stronger effect on well-being for the female caregivers of a perceived lack of improvement in the patient's ability to function independently at Time 2. A left hemisphere site of lesion continued to contribute significantly to explaining the variance in Time 2 negative well-being for the caregivers, accounting for $10.1 \%$ of the variance in the outcome measure. This relationship between left hemisphere site of lesion and lower negative well-being score was seen consistently both at Time 1 and Time 2 . Caregiver level of burden explained an additional $1.8 \%$ of the variance. Neither baseline levels of subjective health and satisfaction with the quality of network contacts, nor changes in these variables over time contributed to 
TABLE LXXIII

HIERARCHICAL REGRESSION FOR NEGATIVE WELL-BEING,

TIME 1 AND TIME 2: FEMALE CAREGIVERS ONLY

Dependent Variable Negative

Well-Being, Time 2

\begin{tabular}{|c|c|c|c|c|c|}
\hline & MULTIPLE R & $\mathbf{R}^{2}$ & $\mathrm{R}^{2}$ CHANGE & SIMPLE R & BETA \\
\hline Negative Well-Being, $T_{1}$ & .666 & .443 & .443 & .666 & 0.274 \\
\hline Subjective Health Rating, $T_{1}$ & .666 & .443 & .000 & -.258 & -0.158 \\
\hline Subjective Health Rating, $\mathrm{T}_{2}$ & .668 & .446 & .002 & -.091 & 0.315 \\
\hline Stroke Severity Rating, $T_{1}$ & .712 & .506 & .061 & .339 & -0.009 \\
\hline Stroke Severity Rating, $\mathrm{T}_{2}$ & .759 & .576 & .070 & .491 & 0.223 \\
\hline $\begin{array}{l}\text { Satisfaction with Quality of } \\
\text { NW Contact, } T_{1}\end{array}$ & .766 & .587 & .010 & -.454 & -0.088 \\
\hline $\begin{array}{l}\text { Satisfaction with Quality of } \\
\text { NW Contact, } T_{2}\end{array}$ & .766 & .587 & .000 & -.390 & -0.025 \\
\hline Percelved Caregiver Burden & .779 & .605 & .018 & .452 & 0.302 \\
\hline Site of Lesion (Left Hemis.) & .840 & .706 & .101 & -.540 & $-0.51 \%$ \\
\hline Site of Lesion (Rt. Hemis.) & .844 & .712 & .005 & .411 & -0.134 \\
\hline
\end{tabular}


predicting the variability in Time 2 negative well-being.

\section{SUMMARY: CHANGE OVER TIME}

Analysis of the distribution of change scores for the outcome measures revealed that except for a few caregivers and patients, depression and well-being scores remained relatively stable over time. The small numbers of subjects in the change groups did not permit meaningful statistical analyses of the correlates of change in depression and wellbeing levels in these subgroups of patients and caregivers. Scores on some of the independent variables did change significantly over time. For the patients, objective health factors and expectations for future health showed negative changes. Caregivers saw significant improvement in the ADL functioning of the patients over time, and became less concerned as a group about the possibility of another stroke. Their overall networks increased in size at Time 2 , but they reported fewer reciprocal task exchanges and a decreased proportion of instrumental help from the close network. Patients indicated an increased likelihood of confiding in their partners at Time 2, while the caregivers reported a decreased likelihood that they would confide in their partner after the stroke.

The best predictor of Time 2 depression level for the caregivers was Time 1 depression score. Perceived caregiver burden also contributed substantially to explaining the 
variance in Time 2 depression. The proportion of reciprocal confiding relationships within the close network before the stroke was less predictive of depression score than the degree of change in this variable over time. Positive wellbeing score at Time 1 explained most of the variance in Time 2 caregiver positive well-being. Other predictors of positive well-being over time were the patient's initial level of ADL functioning, the number of confiding relationships in the pre-stroke network, the caregiver still reporting a relatively high level of marital happiness after the stroke, and change in the number of confiding relationships reported over time. As was true for the other outcome measures, the level of negative well-being experienced at Time 2 was best predicted by the negative mell-being score at Time 1. Site of lesion being in the left hemisphere, stroke severity rating, and perceived caregiver burden also made contributions to explaining the level of negative well-being at Time 2 .

Chapter VIII will discuss the general findings of the study as well as their implications for intervention strategies and future research. 
CHAPTER VIII

DISCUSSION AND IMPLICATIONS OF THE STUDY

DISCUSSION

The model of adaptation to a stroke which was presented in Chapter II included characteristics of the individual, characteristics of the stroke disability, perceptions of the stroke, social network and support factors, and elements of the partner relationship as potentially important predictors of adaptive functioning and well-being in stroke patients and their partner-caregivers.

Based on previous investigations, the model predicted that the severity of the stroke and related functional levels of independence in activities of daily living would have an influence on both partners' well-being. It was also predicted that higher levels of depressive symptomatology would be associated with increased severity of the stroke and with lower levels of functional ADL independence. Site of the stroke lesion (right or left hemisphere) was expected to influence depression and well-being to the degree that the ability to interact in socially appropriate ways was altered. Left hemisphere site of lesion, with its associated effects on the patient's ability to verbally communicate, was expected to result in poorer caregiver outcomes. Feelings of guilt or blame regarding the stroke's occurrence, greater concern about having another stroke or 
about the availability of assistance in the future, and the pexception of the stroke ag seyerely disabling were expected to be associated with higher depression leveis, lower positive well-being, and higher negative well-being. For the caregivers, greater feelings of burden related to caring for the stroke patient were predicted to be related to higher depressive symptom level and less positive wellbeing, as were perceived negative behavior and personality changes in the patient.

Better perceived health status, a lower objective health problem score, a greater level of optimism, and higher degree of religiosity were individual characteristics which were expected to be predictive of better mood and coping for both patients and caregivers.

Previous research on coping with a chronic health condition led to the prediction in the model for this study that pre-stroke levels of social activity and social network integration would be related to depression and well-being outcomes. That is, higher pre-morbid levels of social integration would be associated with lower levels of depression and greater positive well-being.

Based on descriptive studies of the impacts of stroke as well as the investigator's personal experience in working with stroke patients and their families, negative impacts on the social networks of the subjects in this study were expected. It was predicted that social networks would 
diminish in size, and that frequency of contact with network members would be decreased compared to pre-stroke levels. The networks of patients and caregivers were expected to become more dense, or interconnected. Some change in the relationship compositon of the networks in the direction of fewer friends and more relatives was predicted.

Furthermore, based on exchange and reciprocity theory, it was hypothesized that the level of reciprocity within the exchange networks of both patients and caregivers would be reduced after the stroke, and that decreases in reciprocity would be associated with higher negative well-being and depression scores. Based on previous stroke research (McLeroy et al., 1985), research related to other illnesses and disabilities such as cancer (Dunkel-schetter, 1984), recent social network studies (Fiore et al., 1983; Rook, 1984), and on reciprocity theory, some negative impacts of social support and social network interaction were predicted. These predictions were related to expectations that, following a stroke, some network support attempts would be inappropriate, that expectations of support might not always be congruent with actual provisions of support, and that assistance which can no longer be reciprocated might result in negative feelings of well-being.

Aspects of the patient-caregiver relationship which were expected to be related to outcomes for both partners were their individual perceptions of the overall 
relationship quality, the level of perceived communication within the relationship, the amount of shared partner activity, and the reported frequency of marital disagreement. Other factors related to the couple's relationship included the degree of overlap between their social networks, the congruence of their perceptions regarding the stroke, and the impact of one partner's affect, level of optimism, and feeling of well-being on the other member of the dyad. All of these associations were predicted to be in the expected direction. Greater network overlap was predicted to be associated with higher depression and negative well-being levels, and lower positive well-being.

Finally, it was expected that overall the patients would show higher levels of depressive symptoms and of negative well-being, and lower levels of positive well-being than general community samples. For the caregivers, no clear prediction about overall levels of depression and well-being was made.

To explore these questions and test these predictions, data were gathered from 50 partners of recent stroke victims and from the patients themselves when possible. The partners provided information about those patients who were unable to respond. Information was obtained at two points in time, shortly after the stroke occurrence and six months later. This permitted the investigation of changes in all 
of the major variables over time.

\section{Descriptive Results}

First, descriptive analyses of demographic, social network, and social support factors were completed. The results of these descriptive analyses were reported in detail in Chapters IV and V. Few demographic changes were found over time except for the expected decline in the number of patients who were employed after the stroke. About $40 \%$ of the subjects felt that their economic circumstances had declined since the stroke. Significant declines in the reported frequency of religious activity and attendance were found for the caregivers at Time 2, although the overall level of social activity reported did not decrease significantly for either caregivers or patients. overall, the self-reported health status of the caregivers remained stable over time, while that of the patients declined, not unexpectedly. Caregivers became less concerned about the possibility of another stroke at Time 2 . overall, the patients improved significantly in their functional ADL status as rated by their spouses.

This study focused particularly on social network and social support changes related to a first, disabling stroke. Table LXXIV contains a summary of the various social network and social support measures obtained in the study. Some predictions regarding negative changes in the structural and interactional aspects of the social networks 
TABLE LXXIV

SUMMARY OF SOCIAL NETWORK AND SOCIAL SUPPORT MEASURES OBTAINED IN THE STUDY, TIME 1 AND TIME 2

\section{Structural Network Measures}

Size of the Overall Network (up to 20 persons)

Size of the Intimate Network (up to 10 persons)

Gender, Age, and Relational Composition

Network Density and Degree

Network Overlap (between partners' networks)

Geographical Distance of NW Members

Interactional Network Measures

Frequency of Face-to-Face Contacts

Frequency of Other Types of Contacts

\section{Social Integration Measures}

Level of Social Activity

Organizational Memberships

Volunteer Organization Involvement

\section{Social Support Measures}

Amount and Proportion of Instrumental Task Assistance

Amount and Proportion of Illness Help

Amount and Proportion of Coñ̂iding Support

Amount and Proportion of Informational Support

Amount and Proportion of Socializing Support

Total Number and Proportion of Reciprocal Support

Exchanges, by Type of Support

Specific, stroke-Related support

\section{Perceived Quality of Network Support}

Satisfaction with Overall Amount of Support Satisfaction with Overall Quality of support

\section{Neqative Aspects of Support}

Number of Persons Helping Less than Expected

Number of Persons Who Make Things Worse

Number of Persons who Don't Get Along With 
were confirmed by the data, while others were not. The expected declines in oyerall network size after the stroke were not found for the caregivers or for the stroke patients. In fact, the number of network members in the larger network increased significantly for the caregivers. Likewise, data analysis did not reveal any significant declines in the frequency of face-to-face contacts with network members, as had been predicted, although frequency of other types of contacts did decline at Time 2 for both patients and caregivers. Minimal changes over time were seen in the relationship composition of the partners' networks. For the patients, however, the predicted decline in the number and proportion of friends in the network and increase in the number and proportion of relatives in the network was observed at Time 2. Network density and overlap did increase at Time 2 , as expected, but the magnitude of the increase was small.

Some of the predicted changes regarding network social support were confirmed. Caregivers reported both giving and receiving less support from their close network at Time 2 , across types of support. Patients reported receiving more instrumental help from the close network after the stroke, and slightly more confiding help, but less informational assistance. Interestingly, they saw themselves at Time 2 as providing less instrumental aid to members of the network, but more confiding support and informational help. The 
patients in this study appeared to maintain a sense of reciprocity within their networks bi viating themselves as providing more confiding and informational support to network members after the stroke, when they were less able to offer instrumental or task-oriented assistance to others. The patients did not show the expected decline in socializing support at Time 2 , nor did the caregivers. The expected decline in the number and proportion of reciprocal support exchanges did not occur for this sample. Some changes in the direction of support were seen, however. Caregivers saw themselves as providing less confiding support to members of their network at Time 2 , and there was an increase from 16 to $30 \%$ in the proportion of the sample who reported being overhelped in the exchange of confiding support.

With regard to the prevalence of depressive symptoms in the sample, as expected, a fairly high proportion of the group of less communicatively impaired stroke patients had scores indicative of being at high risk for depression. What was not expected was that almost half of the caregivers (48\%) scored above the cutoff level on the CES-D at Time 1. These percentages decreased slightly at Time 2 (to 2j\% for the patients and $45 \%$ for the partners), but remained considerably higher than the proportion of individuals at risk for depression among the general population of elderly persons. Results of this study, then, 
confirmed what previous researchers have suggested: that a chronic, disabling illness has serious negative impacts on the psychological status of the primary caregiver, particularly on the spouse.

Consistent with expectations, the patients' positive well-being scores decreased over time, while their negative well-being scores increased slightly. Caregivers maintained their level of positive well-being at Time 2, while their reported level of negative well-being decreased slightly. Both patients and partners reported scores on the IPWB which were similar to those reported for community samples. The caregivers as well as the patients reported fairly high levels of optimism at Time 1 . Optimism score (LOT) decreased significantly for the caregivers at Time 2 , but not for the patients.

\section{Correlational Results}

Numerous Pearson and Rendall correlation coefficients were computed in order to investigate whether this study confirmed predicted relationships between outcome variables and other factors.

Depression. Health status, both objective and perceived, was expected to be strongly and negatively related to depression score. In fact, results of the study confirmed the frequently found negative relationship between health status and depression. For the caregivers, subjective health rating was the most strongly correlated 
with depression, with objective health score also a correlate of depression level. These associations were 211 in the expected direction of poorer health rating being correlated with a higher depression score. For the patients, a higher objective health index score and less optimistic expectations for future health were both correlated with a higher level of depression.

Several of the predictions made in Chapter II involved stroke-related variables. These proved to be strong correlates of depression. For the caregivers, a higher depression score was associated with greater concern about caring for the patient in the future, a lower level of patient ADL functioning, feeling that they could have done something to prevent the stroke, and a higher overall stroke severity rating. These findings are consistent with those of Unks (1983), who found the perceived severity of the stroke patient's disabilities to be the strongest predictor of spouse well-being.

The patients' depression scores were positively related to feeling that they could have done something to prevent the stroke, and concern about their partner's ability to care for them in the future.

At Time 2, stroke-related variables continued to be related significantly to depression score. For the caregivers, a greater level of perceived burden, along with increased frequency of negative behaviors and personality 
traits in the patient and decreased frequency of positive patient behaviors were correlated with higher depression scores. Stability of personality and behavior characteristics in the patient was related to lower levels of depressive symptoms. Several writers (Binder, 1983; Lezak, 1978a, 1978b; Stein et al.. 1985) have suggested that coping with negative personality and behavior changes in the stroke patient is more stressful than the necessity to provide physical assistance. Both the patients' and the caregivers' ratings of the patient's functional independence were negatively related to the patients' depression scores. Patients who continued at Time 2 to feel that they could have done something to prevent the stroke were more likely to have higher depression scores. Thus, the prediction that stroke severity rating would be related to depression score was generally upheld.

For the caregivers, previous levels of social activity and social support were negatively correlated, as expected, with Time 1 depression level. For the patients, no network support measures were significantly associated with depression at Time 1 , but several aspects of conjugal support, both positive and negative, were correlated with depression score. Frequency of marital disagreement was positively related to depression level. If the partners reported they were more likely to share concerns with the patient before the stroke, the patients were more likely to 
have higher depression scores at Time 1 .

At Time 2, both positive and negative aspects of network relationships were reflected in the correlations of these variables with depression. The number of network members who were difficult to get along with, the amount of overlap between the patient's and caregiver's network, the frequency of face-to-face contacts, and the number of task exchanges were positively correlated with the caregivers' depression scores. These relationships confirmed predictions about the potential negative impacts of the network in the situation of coping with a chronic, disabling iliness. On the other hand, greater network density was associated with lower depression levels at Time 2 for the caregivers, when the predicted relationship was a positive one between density and depression score. Apparently, as other researchers have suggested (Hirsch, 1980), tightly knit networks may serve a necessary support function for older persons, and in the context of a chronic illness, whereas less dense networks may be more beneficial to those experiencing life transitions which are off-time events or which require new sources of information and advice.

Similar results were seen in the pattern of correlations between support measures and patient depression scores. Higher levels of some types of instrumental help. such as transportation assistance, were associated with higher depression scores for the patients. While it is 
possible that this relationship between levels of instrumental support and depression may simply be indicative of the relationship of both higher levels of support and depression score to severity of the stroke disability (Dunkel-schetter, 1984), it is also possible that inappropriate levels of support are responsible for the correlation between support levels and depression. Furthermore, we had predicted that those patients who had previously been more socially integrated and who retained more friends in their network at Time 2 would be functioning better psychologically after the stroke. What was found, however, was that indicators of social connectedness such as the proportion of friends in the network were, in fact, associated with higher depression levels. Possible reasons for these seemingly contradictory findings were discussed in Chapter VI, including the notion of stigma attached to a stroke. As Hyman (1971) found, those stroke patients who had been more socially active prior to their stroke reported more feelings of being stigmatized by their stroke-related problems early in their rehabilitation program, and had poorer rehabilitation outcomes, despite initial functional levels comparable to patients who did not express feelings of being stigmatized. A possible explanation for the fact that those patients in the present study who retained a greater proportion of friends in their networks at Time 2 also reported higher depression scores is that contacts with 
friends reinforced feelings of being stigmatized. This could be because of patient self-perceptions (that is social comparison processes involving the present self compared to friends and former self) or because of reactions of friends to the changed status of the patient (Dunkel-schetter \& Wortman, 1982; Wortman \& Dunkel-Schetter, 1979). Some support for these interpretations is found in the fact that overall satisfaction with the amount network contacts was associated with lower depression scores for the patients. That is, satisfaction with the amount of network contact appeared to be independent of the actual level of social connectedness with friends following the stroke.

\section{We11-Being}

Examination of the correlations between the major independent variables and well-being scores were similar to the findings regarding depression scores. Positive wellbeing for the caregivers was related in the expected direction to a number of measures of social activity and social support, especially at Time 1, including the level of overall social activity before the stroke. This was an expected outcome, and consistent with research findings on the correlates of morale and well-being in older persons. Frequency of network contacts in the immediate post-stroke period was also positively associated with caregiver wellbeing. For the patients, a different picture emerged. Levels of pre-stroke social activity and shared marital 
activity were associated at Time 1 with lower patient positive well-being.

With regard to negative well-being, correlational associations were in the expected direction for health and stroke-related factors except for the relationship between site of lesion and negative well-being. Whereas it had been predicted that a left nemisphere lesion would be related to more negative caregiver well-being, because of the greater incidence of significant communication problems in persons with left hemisphere strokes, just the opposite was found. The patient having had other than a left hemisphere stroke was strongly related to higher negative well-being. Apparently, the affective and behavioral changes frequently associated with right hemisphere strokes were stronger correlates of caregiver well-being than were stroke-related communication problems. As discussed in Chapter 1, the literature on the impacts of stroke has reported contradictory findings concerning the effect on the caregiver of the site of the stroke lesion. Artes and Hoops (1976), Fengler and Goodrich (1979), and Kinsella and Duffy (1979, 1980) reported the spouses of left-hemisphere damaged patients with aphasia to be doing less well psychosocially than the wives of right-hemisphere damaged patients without aphasia. On the other hand, Coughlan and Humphrey (1982) found that the partners of individuals with right hemisphere strokes and hemiplegia were more likely to report decreased 
enjoyment of life. The psychological impacts on the spouse were not measured in the same way in any of these studies, and none of them used standardized measures of psychological well-being, as was done in the present study, which may partially explain the differences in results. Also, the severity of the communication problem and the presence or absence of hemiplegia may have been factors which influenced results.

Stroke-related concerns and objective health problems were more strongly related to negative well-being for the patients than were network or conjugal support variables, although support was also important. As was seen for depression and for positive well-being, having been more socially integrated before the stroke was associated with higher negative well-being for the patients at Time 1 .

At Time 2, health, network support, and marital support factors were all significantly associated in the expected direction with positive well-being for the caregivers. For the patients, maintaining a high level of shared marital activity after the stroke was associated with higher positive well-being. Correlates of negative wellbeing for the caregivers, all in the expected direction, were stroke-related factors, especially degree of perceived burden, satisfaction with the amount and quality of network contact, network density, and several other measures of social integration and support. Several aspects of the 
marital relationship were also predictably related to the caregivers'negative weli-being scores. Clearly, the level of pre-stroke social connectedness that was maintained over time had positive effects on the caregivers' well-being. These results confirm the findings of previous studies regarding the impacts of perceived burden (zarit et al., 1980; Zarit et al, in press), the importance of the perceived adequacy of network support (Cohen et al., 1984; Turner et al., 1983), and the importance of socializing contacts (Fiore et al, 1983; Rook, 1984).

Again, the opposite pattern was seen for the patients. At Time 2, as had been observed at Time 1, patients who reported higher levels of social activity and who had a greater proportion of friends in their network were more likely to have higher negative well-being scores. On the other hand, greater network density, and satisfaction with the amount of network contact were correlated with lower negative well-being scores. Health factors were importantly related to negative well-being for the patients, especially a perceived negative change in health status from Time 1 to Time 2.

To summarize the correlational results, predicted relationships were found between health status and the outcome measures, and between the stroke-related variables and the outcome measures. Some of the expected relationships between social network and support factors and 
well-being and depression were confirmed, while others were not. A number of negative network impacts on the paitients and the caregivers were suggested by the correlational results.

\section{Results of Regression Analyses}

For the caregivers, a series of regression analyses explored the best predictors of depression and well-being at Time 1 and Time 2, and over time. These analyses were carried out with all of the caregivers included as well as with only the female caregivers included.

Time 1. As has been found in many previous studies, the best predictor of caregiver depression score at Time 1 was subjective health rating. Subjective health status is gobal construct which has repeatedly been shown to be predictive of and related to psychological well-being in the general population. The patient's level of ADL functioning, concern about being able to care for the patient in the future, and the proportions of instrumental task assistance and reciprocal confiding within the pre-stroke network were also independently predictive of depression score. These results are consistent with the hypotheses stated in Chapter II.

Positive well-being was best predicted by the patient's level of functioning in activities of daily living, together with a number social network and support factors: the level of pre-stroke social activity, the 
degree of shared marital activity, the number of task exchanges in the pre-stroke network, and the overail rating of the quality of the partner relationship before the stroke.

The best predictor of negative well-being at Time 1 was subjective health rating, followed by the site of lesion being in the right hemisphere, satisfaction with the quality of network contacts, and overall rating of marital happiness.

Time 2. The strongest predictor of depression score at Time 2 was the degree of perceived caregiver burden. objective health rating, and network density also added to the explained variance in depression score.

The number of confiding exchanges with network members, subjective health rating, marital happiness rating, and perceived severity of the stroke were the best predictors of positive well-being at Time 2, although together they only accounted for $29 \%$ of the variance in PSWB score.

Negative well-being at Time 2 for the caregivers was best predicted by the perceived severity of the stroke, followed by satisfaction with the amount of network contact, perceived caregiver burden, and network density.

Predictors over Time. The level of depressive symptomatology at Time 1 was the best predictor of Time 2 depression score, as was expected. After controlling for 
subjective health and the patient's level of ADL functioning at Time 1 as well as changes in these factors over time, perceived caregiver burden at Time 2 was a strong predictor of depression score. The proportion of reciprocal confiding exchanges in the network at Time 2, after accounting for the level of pre-stroke network confiding, also explained a substantial amount of the variance in Time 2 depression. The patient's level of ADL functioning at Time 1 contributed to the variance in Time 2 depression as well.

Time 2 positive well-being score was best predicted by Time 1 positive well-being score, followed by the number of confiding exchanges at Time 1, Barthel Index score at Time 1. change in the number of confiding exchanges over time, and changes in perceived marital happiness over time.

Similarly, negative well-being score at Time 1 accounted for most of the explained variance in Time 2 negative well-being score. After controlling for subjective health rating, stroke severity rating, satisfaction with the quality of network contacts, and degree of perceived caregiver burden, a left hemisphere site of lesion accounted for $8 \%$ of the variance in negative well-being. As previously discussed, a left hemisphere stroke was was predictive of a lower negative well-being score. Change in perceived stroke severity at Time 2, stroke severity at Time 1. and perceived burden also added to the prediction of caregiver negative well-being score at Time 2 . 
In conclusion, results of the regression analyses confirmed the importance of some social network and social support factors in explaining adaptation to a chronic, disabling illness. The results also supported the importance of subjective health factors, perceptions of stroke severity, the degree of felt burden, and partner support in predicting depression and well-being in the caregivers over time.

\section{IMPLICATIONS OF THE STUDY}

The findings of this study have implications for those health care practitioners dealing directly with stroke patients and their families, and for those planning and developing intervention programs. First, this study clearly confirms that depression and negative feelings of well-being are not infrequent or isolated occurrences even in a sample of relatively independently functioning stroke patients who are living at home. While a number of recent studies have suggested that depressive symptoms are neither recognized nor treated as frequently as they should be in people who have suffered strokes (Robinson, 1986), this information needs to be more widely disseminated. Furthermore, depression scores in this sample of caregivers and patients fluctuated as much as 30 points in either direction over time. Initial assessment that the patient and caregiver are doing well or poorly may not be predictive of how they will 
be coping in six months or a year's time. Subtantial changes in depression scores over time in both positive and negative directions were observed for about one quarter of the sample. The need for a periodic or continuing contact with these families is underlined by the results of this study .

The fact that almost half of the caregivers remained at high risk for depression at six to eight months after the stroke points up the need for treating the partner as well as the patient. Caregiver support groups have become increasingly available in recent years, but the need for specific outreach to distressed caregivers by health care professionals should be recognized. Of the caregivers in this study, very few were aware of stroke support groups in their area at Time 2 , and almost none had attended any support group or stroke club meetings. Yet these informal support groups exist throughout the metropolitan area. Referral for individual counseling and mental health assistance for those caregivers at risk for depression should occur more frequently than it appears to happen at present.

Specific outcomes of the study have important implications for the content of counseling with stroke families. Basic information about stroke and the causes of stroke may not alleviate the feelings of guilt or blame that a number of the patients and some caregivers expressed, but 
these feelings need to be dealt with throughout the course of rehabilitation and in follow-up visits. Basic information regarding preventive measures and medications to prevent the occurrence of another stroke, repeated as often as necessary, may help to alleviate the concerns of caregivers and patients. Medical advances in the treatment and prevention of the causes of stroke need to be communicated to these families. A high proportion of respondents at Time 2 indicated that the fear of another stroke was their greatest worry or concern.

While there is a tendency to view patients and caregivers with large support systems and a high level of social activity as coping well after a stroke, the possible incongruity between the partner's need to maintain social contacts and the apparent distress that some patients feel in social interaction situations should be explored. Including close friends and family members other than the spouse in counseling may help them to understand that seemingly supportive interactions with the patient and caregiver can have negative effects as well as positive ones. More positive ways of interacting and supporting the stroke patient could be taught and modelled for these patients.

One of the important findings of the study related to social network support was that the caregivers perceived a decline in reciprocity in their network exchanges after the 
stroke. In addition, change in the proportion of reciprocal confiding exchanges was found to be an important predictor of caregiver depression score at Time 2. Many researchers have emphasized the importance of maintaining reciprocal helping relationships to an individual's sense of wellbeing. One of the aspects of caregiver support that needs to be emphasized in intervention models is the maintenance of a sense of reciprocity for the caregiver, and the fostering of substitute confiding relationships if the stroke patient is no longer able to fulfill that role within the marriage. Indeed, caregiver support groups themselves may at least partially serve this function.

Finally, the families in this study were not frequent users of community assistance and other formalized support programs either before or after the stroke. In response to open-ended questions at the end o: the Time 2 interview, however, they did mention several types of services which they had found difficult to obtain and which would be helpful. Most frequently mentioned were the need for occasional in-home assistance, and respite for the caregivers. Assistance with transportation was another important unmet need. Results of the correlational analysis suggested that continually depending on the close network for transportation help was in some ways stressful for both the patients and the partners. Other services mentioned as desirable and needed were more recreation opportunities for 
the patients, and some sort of continued therapy contacts, even if just for periodic re-evaluations.

Current Medicare and other third party reimbursement policies are structured so that acute stroke care and early, intensive physical rehabilitation are supported, but followup and maintenance therapy are not reimbursed. Yet, one of the more effective ways of assisting the stroke patient to develop more positive social comparisons and to deal with feelings of stigma may be ongoing therapy groups. The nature of stroke-related psychosocial adjustment problems, and the impacts of these problems on both the patient and the caregiver need to be reflected in health insurance reimbursement policies. Stroke is a chronic illness which demands ongoing follow-up and long-term supportive interventions.

In conclusion, this sample of community dwelling stroke patients and their partner-caregivers were, on the whole, coping reasonably well at six months to eight months after the occurrence of a disabling stroke. Nevertheless, a substantial proportion remained at risk for depression and about one-third of the caregivers reported high levels of perceived burden related to the stroke. While the, overall social networks and amount of social support appeared to remain intact, the results of this study indicated qualitative changes in some subjects' interactions with their support systems. Finally, the generally optimistic 
picture suggested by the findings of this study were not true for a subgroup of about 20 to $25 \%$ of the couples, and should not be generalized to more impaired stroke samples, or patients without partner support. Future research on the process of adaptation to a stroke over time for those stroke patients without partner support, or who are not dwelling in the community would be a logical extension of this study. Specific intervention studies involving patients, partners, and members of the close network and incorporating control groups are needed. To date, descriptions of stroke intervention programs have lacked both data to substantiate claims of positive outcomes and the incorporation of control groups. Such intervention studies, if focused on the caregivers, might work on enhancing the utilization of available support, on increasing the opportunities for reciprocal support exchanges for the patient and the caregiver, and on providing basic information to alleviate stroke-related anxieties and concerns. Patient-focused interventions might concentrate on specific strategies for encouraging more appropriate social comparisons, for reducing feelings of stigma, and for utilizing the network more effectively in order to maintain network support. 
Abbey, A., Abramis, D. J., \& Caplan, R. D. (1985). Effects of different sources of social support and social conflict on emotional well-being. Basic and Applied Social Psychology, $6,111-129$.

Ahlsio, B., Britton, M., Murray, V., \& Theorell, T. (1984). Disablement and quality of life after stroke. Stroke, 15. 886-890.

Anderson, E., Anderson, T. P. \& \& Rottke, F. J. (1977). Stroke rehabilitation: Maintenance of achieved gains. Archives of Physical Medicine and Rehabilitation, $\underline{58}$, 345-352.

Anderson, T. P., \& Kottke, F. J. (1978). Stroke rehabilitation: A reconsideration of some common attitudes. Archives of Physical Medicine and Rehabilitation, 59, 175-181.

Andrews, R., \& Stewart, J. (1979). Stroke recovery: He can but does he? Rheumatology and Rehabilitation, 18, 43-48.

Antonovsky, A. (1979). Health, stress, and coping. San Francisco: Jossey-Bass.

Antonucci, T. C., \& Depner, C. E. (1982). Social support and informal helping relationships. In T. A. Wills (Ed.), Basic processes in helping relationships. New York: Academic Press.

Arling. G. (1976). The elderly widow and her family, neighbors, and friends. Journal of Marriage and the Family, 38, 757-768.

Artes, R., \& Hoops, R. (1976). Problems of aphasic and nonaphasic stroke patients as identified and evaluated by patients' wives. In Y. Lebrun and R. Hoops (Eds.). Recovery in aphasics. Amsterdam: Swets and Zeitlinger B. V.

Barrera, M. (1981). Social support in the adjustment of pregnant adolescents: Assessment issues. In $B$. $H$. Gottlieb (Ed.), Social networks and social support. Beverly Hills, CA: Sage Publications.

Baum, H. M. (1982). Stroke prevalence: An analysis of data from the 1977 National Health Interview Survey. Public Health Reports, 97, 24-30. 
Baum, H. M., \& Robins, M. (1981). The National Survey of Stroke: Survival and prevalence. Stroke, 12 (Part 2, SuppI.1), I-59-I-6े.

Beck, A. T. (1967). Depression: Clinical, experimental, and theoretical aspects. New York: Harper \& Row, 1967 .

Beck, A. T., Ward, C. H., Mendelson, M., Mock, J., \& Erbaugh, J. (1961). An inventory for measuring depression. Archives of General Psychiatry, 4. 561571 .

Beck, A. T., Weissman, A., Lester, D., \& Trexler, L. (1974). The measurement of pessimism: The hopelessness scale. Journal of Consulting and Clinical Psychology, 42, 861-865.

Becker, C., Howard, G., McLeroy, R. R., Yatsu, F. M., Toole, J. F., Coull, B., Feibel, J., \& Walker, M. D. (in press). Community hospital-based stroke programs: North Carolina, Oregon and New York. II. Description of study population. Stroke.

Belcher, S. A., Clowers, M. R., \& Cabanayan, A. C. (1978). Independent living rehabilitation needs of postdischarge stroke persons: A pilot study. Archives of Physical Medicine and Rehabilitation, 59 . $404-409$.

Belcher, S. A., Clowers, M. R., Cabanayan, A. C., \& Fordyce, W. E. (1982). Activity patterns of married and single individuals after stroke. Archives of Physical Medicine and Rehabilitation, $63,308-312$.

Belle, D. (1982). The stress of caring: Women as providers of social support. In L. Goldberger \& $S$. Breznitz (Eds.), Handbook of stress. New York: The Free Press.

Berkman, L. F., \& Syme, S. L. (1979). Social networks, host resistance, and mortality: A nine year follow-up study of Alameda County residents. American Journal of Epidemiology, 109, 186-204.

Berkman, P. (1971). Measurement of mental health in a general population survey. American Journal of Epidemiology, 94, 105-111. 
Berle, B. B., Pinsky, R. H., Wolf, S., \& Wolff, H. G. (1952). A clinical guide to prognosis in stress disease. Journal of the American ifedical Association, 149, 1624-1628.

Binder, L. M. (1983). Emotional problems after stroke. Current Concepts of Cerebrovascular Disease: stroke, 18, 17-21.

Blazer, D. G. (1982). Social support and mortality in an elderly community population. American Journal of Epidemiology, 115, 684-694.

Bloom, J. R., \& Spiegel, D. (1984). The relationship of two dimensions of social support to the psychological well-being and social functioning of women with advanced breast cancer. Social Science and Medicine, 19. $831-837$.

Boissevain, J. (1974). Friends of friends. New Yort:: St. Martin's Press.

Bott, E. (1957). Family and social network. London: Tavistock Publications.

Bradburn, N. M. (1969). The structure of psychological well-being. Chicago: Aldine.

Bradburn, N., \& Caplovitz, D. (1965). Reports on happiness. Chicago: Aldine.

Brocklehurst, J. C., Morris, P., Andrews, K., Richards, B., \& Laycock, P. (1981). Social effects of stroke. Socizl Science and Medicine, 15A, 35-39.

Brody, E. L. (1977). Long-term care of older people: A practical quide. New York: Human Sciences Press.

Brown, G. W., \& Harris, T. (1978). Social origins of depression: A study of psychiatric disorder in women. New York: Free Press.

Brown, J. S., Rawlinson, M. E., \& Harden, D. (1982). Family functioning and health status. Journal of Family Issues, $3,111-132$.

Buck, M. (1968). Dysphasia: Professional quidance for Eamily and patients. Englewood Cliffs, NJ: PrenticeHall.

Burish, T. G., \& Bradley, L. A. (Eds.). (1983). Coping with chronic disease. New York: Academic Press. 
Campbell, A. (1981). The sense of well-being in America. New York: McGraw-Hill.

Caplan, G. (1974). Support systems and community mental health. New York: Behavioral Publications.

Cassel, J. (1976). The contribution of the social environment to host resistance. American Journal of Epidemiology, 104, 107-123.

Cassel, J. (1974). Psychosocial processes and "stress": Theoretical formulation. International Journal of Health Services, 4, 471-482.

Cassem, N. H. \& Hackett, T. P. (1971). Psychiatric consultation in a coronary care unit. Annals of Internal Medicine, 75, 9-14.

Cassileth, B., Lusk, E., Strouse, T., Miller, D., Brown, L., Cross, P., \& Tenaglia, A. (1984). Psychosocial status in chronic illness, a comparative analysis of six diagnostic groups. The New England Journal of Medicine, 311, 506-511.

Cay, E., Vetter, N., Philip, A., \& Dugard, P. (1972). Psychological status during recovery from an acute heart attack. Journal of Psychosomatic Research, 16, 425-435.

Chapman, N. J., Pancoast, D. L., \& Parker, P. (1983). Supporting caregivers of the elderly. Final report to the Northwest Area Foundation. Regional Research Institute for Human Services, Portland State University, Portland, Oregon.

Chwat, S., Chapey, R., Gurland, G., \& Pieras, G. (1980). Environmental impact of aphasia: The child's perspective. In $R$. Brookshire (Ed.), Clinical Aphasiology Conference Proceedings, 1980. Minneapolis, MN: BRR Publishers.

Cobb. S. (1976). Social support as a moderator of life stress. Psychosomatic Medicine, 38, 300-314.

Cohen, C. (1978). Three-year follow-up study of stroke patients at the Medical College of Virginia. Southern Medical Journal, 71, 930-932. 
Cohen, L. H., McGowan, J., Fooskas, S., \& Rose, S. (1984). Positive life events and social support and the relationship between life stress and psychological disorder. American Journal of Community Psychology. 12. 567-587.

Cohen, S., Kamarck, T., \& Mermelstein, R. (1983). A global measure of perceived stress. Journal of Health and Social Behavior, 24, 385-396.

Cohen, S.. \& Wills, T. A. (1985). Stress, social support, and the buffering hypothesis. Psychological Bulletin, 98, 310-357.

Comstock, G. W., \& Helsing, R. J. (1976). Symptoms of depression in two communities. Psychological Medicine, 6, 551-563.

Connor, K. A., Powers, E. A., \& Bultena, G. L. (1979). Social interaction and life satisfaction: An empirical assessment of late life patterns. Journal of Gerontology, 34, 116-121.

Coughlan, A. R., \& Humphrey, M. (1982). Presenile stroke: Long-term outcome for patients and their families. Rheumatology and Rehabilitation, 21, 115-122.

Cronbach, L. J. (1951). Coefficient alpha and the internal structure of tests. Psychometrika, 16, 297-334.

Cronbach, L. J., \& Furby, L. (1970). How should we measure "change" - or should we? Psychological Bulletin, 74. $68-80$.

Croog, S. H., \& Levine, S. (1982). Life after a heart attack. New York: Human Sciences Press.

Croog, S. H., Lipson, A., \& Levine, S. (1972). Help patterns in severe illness: The roles of kin network, non-family resources, and institutions. Journal of Marriage and the Family, 34, 32-41.

Crowne, D., \& Marlowe, D. (1960). A new scale of social desirability independent of psychopathology. Journal of Consulting Psychology, 24, 349-354.

Crowne, D. P., \& Marlowe, D. (1964). The approval motive: Studies in evaluative dependence. New York: Wiley. 
Cutler, M. E., \& Harootyan, R. A. (1975). Demography of the aged. In D. S. Woodruff \& J. E. Birren (Eds.), Aging: Scientific parspectives and social issues. New York: Van Nostrand.

Davidson, T. N., Bowden, M. L. \& Feller, I. (1981). Social support and post-burn adjustment. Archives of Physical Medicine and Rehabilitation, 62, 274-278.

Dean, A., \& Lin, N. (1977). The stress-buffering role of social support. Journal of Nervous and Mental Disease, 65, 403-417.

DeJong, G., \& Branch, L. G. (1982). Predicting the stroke patient's ability to live independently. Stroke, 13 , 648-655.

Depner, C., \& Ingersoll-Dayton, B. (1985). Conjugal social support: Patterns in later life. Journal of Gerontology, 40, 761-766.

Dhooper, S. S. (1984). Social networks and support during the crisis of heart attack. Health and Social Work, g. 294-303.

Diener, E. (1984). Subjective well-being. Psychological Bulletin, 95, 542-575.

DiMatteo, M. R., \& Hays, R. (1981). Social support and serious illness. In B. H. Gottlieb (Ed.), Social networks and social support. Beverly Hills, CA: Sage Publications.

Dimond, M. (1979). Social support and adaptation to chronic illness: The case of maintenance hemodialysis. Research in Nursing and Health, $2,101-108$.

Doehrman, S. (1977). Psycho-social aspects of recovery from coronary heart disease: A review. Social science and Medicine, 11, 199-218.

Dohrenwend, B. S., \& Dohrenwend, B. P., (1981). Stressful life events and their contexts. New York: Prodist.

Donaldson, S. W. Wagner, C. C. \& Gresham, G. E. (1973). A unified ADL evaluation form. Archives of Physical Medicine and Rehabilitation, 54, 175-179; 185 .

Dunke1-Schetter, C. (1984). Social support and cancer findings based on patient interviews and their implications. Journal of Social Issues, 40(4), 77-98. 
Dunkel-Schetter, C., \& Wortman, C. B. (1981). Dilemmas of social support: Parallels between victimization and aging. In S. B. Kiesler, J. M. Morgan, \& V. R. Oppenheimer (Eds.), Aging: Social change. New York: Academic Press.

Dunkel-Schetter, C., \& Wortman, C. B. (1982). The interpersonal dynamics of cancer: Problems in social relationships and their impact on the patient. In $H$. S. Friedman \& M. R. DiMatteo (Eds.), Interpersonal issues in health care. New York: Academic Press.

Durkheim, E. (1951). Suicide (J. A. Spaulding \& G. Simpson, Trans.). New York: The Free Press. (Original work published 1897).

Eckenrode, J., \& Gore, S. (1981). Stressful events and social supports: The significance of context. In $B$. H. Gottlieb (Ed.), Social networks and social support. Beverly Hills, CA: Sage Publications.

Evans, R. L., \& Northwood, L. R. (1979). The utility of natural help relationships. Social science and Medicine, 13A, 789-795.

Feibel, J. H., \& Springer, C. J. (1982). Depression and failure to resume social activities after stroke. Archives of Physical Medicine and Rehabilitation, 63. 276-278.

Feldman, D. (1974). Chronic disabling illness: A holistic view. Journal of Chronic Disease, 27, 287-291.

Fengler, A. P., \& Goodrich, N. (1979). Wives of elderly disabled men: The hidden patients. The Gerontologist, $19,175-183$.

Fenigstein, A., Scheier, M. F., \& Buss, A. H. (1975). Public and private self-consciousness: Assessment and theory. Journal of Consulting and Clinical Psychology, 43, 522-527.

Finlayson, A. (1976). Social networks as coping resources: Lay help and consultation patterns used by women in husbands' post-infarction career. Social science and Medicine, 10, 97-103.

Fiore, J., Becker, J., \& Coppel, D. B. (1983). Social network interactions: A buffer or a stress. American Journal of Community Esychology, 11, 423-439. 
Fischer, C. (1982). To dwell among friends. Chicago: University of Chicago Press.

Frankel, G. \& \& Turner, R. J. (1983). Psychological adjustment in chronic disability: The role of social support in the case of the hearing impaired. Canadian Journal of Sociology, 8, 273-291.

Freese, A. S. (1980). Stroke: The new hope and the new help. New York: Random House.

Funch, D. P., \& Marshall, J. (1983). The role of stress, social support, and age in survival from breast cancer. Journal of Psychosomatic Research, 27, 77-83.

Funch, D. P., \& Mettlin, C. (1982). The role of support in relation to recovery from breast surgery. Social Science and Medicine, 16, 91-98.

Garraway, M. (1985). stroke rehabilitation units: Concepts, evaluation, and unresolved issues. Stroke, 16, 178-181.

Garraway, พ. M., Whisnant, J. P. \& Drury, I. (1983). The continuing decline in the incidence of stroke. Mayo Clinic Proceedings, 58, 520-523.

Garraway, W. M., Whisnant, J. P., Kurland, L. T., \& O'Falion, พ. M. (1979). Changing pattern of cerebral infarction, 1945-1974. Stroke, 10, 657-663.

Gilleard, C. J. (1984). Problems posed for supporting relatives of geriatric and psychogeriatric day patients. Acta Psychiatrica Scandinavia, 70, 198-208.

Goldberg, E. (1981, October). Health effects of becoming widowed. Paper presented at a symposium: The changing risk of disease in women. Baltimore, MD: Johns Hopkins University.

Goldberg, E. L., Vannatta, P., \& Comstock, G. W. (1985). Depressive symptoms, social networks and social support of elderly women. American Journal of Epidemiology, 121, 448-456.

Gordon, W. A., \& Diller, L. (1983). Stroke: Coping with a cognitive deficit. In T. G. Burish \& L. A. Bradley (Eds.), Coping with chronic disease. New York: Academic Press. 
Gore, S. (1981). Stress-buffering functions of social supports: An appraisal and clarification of research methods. In B. S. Donrenwend $\dot{\&} B$. $P$. Donrenwend (Eds.), Stressful life events and their contexts. New York: Prodist.

Gottlieb, B. (1981). Social support strategies. Beverly Hills, CA: Sage Publications.

Gouldner, A. W. (1960). The norm of reciprocity: A preliminary statement. American Sociological Review, 25, 161-178.

Granger, C. V., Greer, D. S., Liset, E., Coulombe, J.. \& O'Brien, E. (1975). Measurement of outcomes of care for stroke patients. Stroke, $\underline{6}, 34-41$.

Gresham, G. E., Fitzpatrick, T. E., Wolf, P. A., McNamara, P. M., Rannel, W. B., \& Dawber, T. R. (1975). Residual disability in survivors of stroke - the Framingham study. The New England Journal of Medicine, 293, 954-956.

Griffith, J. (1985). Social support providers: Who are they? Where are they met? and the relationship of network characteristics to psychological distress. Basic and Applied Social Psychology, 6 , 41-60.

Hall, A., \& Hellman, B. (1985). Social structure, social networks, and social support. In $S$. Cohen \& S. L. Syme (Eds.), Social support and health. New York: Academic Press.

Heitzmann, C. A. \& Raplan, R. M. (1984). Interaction between sex and social support in the control of type II diabetes mellitus. Journal of Consulting and Clinical Psychology, 52, 1087-1089.

Heller, R. (1979). The effects of social support: Prevention and treatment implications. In A. P. Goldstein \& F. H. Ranfer (Eds.), Maximizing treatment gains: Transfer enhancement in psychotherapy. New York: Academic Press.

Henderson, S., \& Byrne, D. (1977). Towards a method for assessing social support systems. Mental Health and Society, $4,164-170$.

Hirsch, B. J. (1980). Natural support systems and coping with major life changes. American Journal of Community Psychology, 8, 159-172. 
Hobfoll, S. E., \& Walfisch, S. (1984). Coping with a threat to life: A longitudinal study of self-concept, social support, and psychological distress. American Journal of Community Psychology, 12, 87-100.

Holbrook, M. (1982). Stroke: Social and emotional outcome. Journal of the Royal College of Physicians of London. 16, 100-104.

Holmes, T. H., \& Rahe, R.H. (1967). The Social Readjustment Rating Scale. Journal of Psychosomatic Research, 11, 213-218.

Horenstein, S. (1970). Effects of cerebrovascular disease on personality and emotionality. In A. L. Benton (Ed.), Behavioral Change in Cerebrovascular Disease. New York: Harper and Row.

House, J. S., \& Rahn, R. L. (1985). Measures and concepts of social support. In $S$. Cohen \& S. L. Syme (Eds.), Social support and health. New York: Academic Press.

House, J. S., Robbins, C., \& Metzner, H. L. (1982). The association of social relationships and activities with mortality: Prospective evidence from the Tecumseh Community Health Study. American Journal of Epidemiology, 116, 123-140.

Hul1, C. H., \& Nie, N. H. (1981). SPSS update 7-9. New York: MCGraw-Hill.

Hyman, M. D. (1972). Social psychological determinants of patients ' performance in stroke rehabilitation. Archives of Physical Medicine and Rehabilitation, 53. 217-226.

Hyman, M. D. (1971). The stigma of stroke. Geriatrics, 26, 132-141.

Isaacs, B. (1982). The continuing needs of stroke patients. In F. C. Rose (Ed.), Advances in stroke therapy. New York: Raven Press.

Isaacs, B., Neville, Y., \& Rushford, I. (1976). The stricken: The social consequences of stroke. Age and Ageing, 5, 188-192.

Rahn, R. L., \& Antonucci, T. C. (1980). Convoys over the life course: Attachment, roles, and social support. In P. B. Baltes \&O. G. Brim (Eds.), Life-span development and behavior (vol. 3). New York: Academic Press. 
Kazak, A. E., \& Wilcox, B. L. (1984). The structure and function of social support networks in families with handicapped children. American Journal of Community Psychology, 12, 645-661.

Ressler, R. C., \& Mcleod, J. D. (1985). Social support and mental health in community samples. In $S$. Cohen \& $S$. L. Syme (Eds.), Social support and health. New York: Adademic Press.

Rinsella, G. J., \& Duffy, F. D. (1980). Attitudes towards disability expressed by spouses of stroke patients. Scandinavian Journal of Rehabilitation Medicine, 12, 73-76.

Rinsella, G. J., \& Duffy, F.D. (1979). Psychosocial readjustment in the spouses of aphasic patients. Scandinavian Journal of Rehabilitation Medicine, 11, 129-132.

Kinsella, G. J., \& Duffy, F. D. (1978). The spouse of the aphasic patient. In $Y$. Lebrun \& R. Hoops (Eds.), The management of aphasia. Amsterdam: Swets and Zeitlinger B. V.

Rlein, R. F., Dean, A., \& Bogdonoff, M. D. (1967). The impact of illness upon the spouse. Journal of Chronic Disease, 20, 241-248.

Rrantz, D., \& Schulz, R. (1980). A model of life crisis, control, and health outcomes: Cardiac rehabilitation and relocation of the elderly. In A. Baum \& J. E. Singer (Eds.), Advances in environmental psychology (vol. 2). Hillsdale, NJ: Erlbaur.

Ruller, L. H. (1978). Epidemiology of stroke. Advances in Neurology, 19, 281-311.

Labi, M. L. C., Phillips, T. F., \& Gresham, G. E. (1980). Psychosocial disability in physically restored longterm stroke survivors. Archives of Physical Medicine and Rehabilitation, 61, 561-565.

Lambert, V. A. (1984). Relationships among the factors psychological well-being, social support, functional status, and demographic characteristics of rheumatoid arthritic women. Nursing Research, 33, 50-51 (Abstract).

Langner, T. S. (1962). A twenty-two item screening score of psychiatric symptoms indicating impairment. Journal of Health and Human Behavior, $\underline{3}, 269-276$. 
Langaer, T. S., \& Michael, S. T. (1963). Life stress and mental health. New York: Macmillan.

LaRocco, J., House, J. S., \& French Jr., J. (1980). Social support, occupational stress, and health. Journal of Health and Social Behavior, 21, 202-219.

Lawrence, L., \& Christie, D. (1979). Quality of life after stroke: A three-year follow-up. Age and Ageing, $\underline{8}$, 167-172.

Lezak, M. (1978a). Living with the characterologically altered brain injured patient. Journal of Clinical Psychiatry, 39, 592-598.

Lezak, M. D. (1978b). Subtle sequelae of brain damage. American Journal of Physical Medicine, 57, 9-15.

Litwak, E., \& Szelenyi, I. (1969). Primary group structures and their functions: Rin, neighbors, and friends. American Sociological Review, 34, 465-481.

Lowenthal, M. F., \& Haven, C. (1968). Interaction and adaptation: Intimacy as a critical variable. American Sociological Review, 33, 20-30.

Lubin, B. (1967). Manual for the depression adjective check lists. San Diego, CA: Educational and Industrial Testing Service.

Mackay, A., \& Nias, B. C. (1979). Strokes in the young and middle aged: Consequences to the family and to society. Journal of the Royal college of Physicians of London, 13, 106-112.

Maddi, S. R., Robasa, S. C., \& Hoover, M. (1979). An alienation test. Humanistic Psychology, 19, 73-76.

Mahoney, F. I., \& Barthel, D. W. (1965). Functional evaluation: The Barthel Index. Maryland State Medical Journal, 14, 61-65.

Maida, C. A., Wolcott, D. L., Ratz, A. H. \& Nissenson, A. R. (1983, February). The contribution of social networks to psychosocial adaptation of CAPD patients. Paper presented at the third annual Social Networks Conference, San Diego, CA.

Malone, R. L. (1969). Expressed attitudes of families of aphasics. Journal of Speech and Hearing disorders, 34, 146-151. 
Marmot, M. \& \& Syme, S. L. (1976). Acculturation and coronary heart disease in Japanese-Americans. American Journal of Epidemiology, 104, 225-246.

Matarazzo, J. D. (1982). Behavioral health's challenge to academic, scientific, and professional psychology. American Psychologist, 37, 1-14.

MeIvor, G. P., Riklan, M., \& Reznikoff, M. (1984). Depression in multiple sclerosis as a function of length and severity of illness, age, remissions, and perceived social support. Journal of Clinical Psychology, 40, 1028-1033.

Mcleroy, R. R., DeVellis, R., DeVellis, B., Raplan, B., \& Toole, J. (1984). Social support and physical recovery in a stroke population. Journal of Social and Personal Relationships, 1, 395-413.

Medalie, J. H. \& Goldbourt, U. (1976). Angina pectoris among 10,000 men: II. Psychosocial and other risk factors as evidenced by a multivariate analysis of a five year incidence study. The American Journal of Medicine, 60, 910-921.

Miller, J. F. (1983). Coping with chronic illness: Overcoming powerlessness. Philadelphia: F. A. Davis.

Minkler, M. \& Biller, R. P. (1979). Role shock: A tool for conceptualizing stresses accompanying disruptive role transitions. Human Relations, $\underline{32}, 125-140$.

Mitchell, J. C. (1969). The concept and use of social networks. In J. C. Mitchell (Ed.), Social networks in urban situations. Manchester, England: University of Manchester Press.

Mitchel1, R. E., \& E. J. Trickett (1980). Task force report: Social networks as mediators of social support. Community Mental Health Journal, 16, 27-44.

Moos, R., \& Tsu, V. D. (1977). The crisis of physical illness: An oveiview. In R. H. Moos (Ed.), Coping with physical illness. New York: Plenum.

Morgan, M., Patrick, D. L., \& Charlton, J. R. (1984). Social networks and psychosocial support among disabled people. Social Science and Medicine, 19, 489-497. 
National Decision Systems. (1982). 1980-U. S. census population and housing characteristics, Vol. 1. San Diego, CA: Author.

New, P. R.. Ruscio, A. T., Priest, R. P., Petritsi, D., \& George, L. A. (1968). The support structure of heart and stroke patients. Social Science and Medicine, $\underline{2}$, 185-200.

Nie, N. H., Hull, C. H., Jenkins, J. G., Steinbrenner, R., \& Bent, D. H. (1975). Statistical package for the social sciences (second ed.). New York: McGraw-Hill.

Noh, S., Wood, D. W., \& Turner, R. J. (1984, November). Depression among the physically disabled: Somatic and psychological contributions. Paper presented at 112 th annual meeting of the American Public Health Association, Anaheim, CA.

Nuckolls, R. B., Cassel, J., \& Raplan, B. H. (1972). Psychosocial assets, life crisis, and the prognosis of pregnancy. American Journal of Epidemiology, 95, 431441 .

Overs, R. P., \& Healy, J. R. (1973). Stroke patients: Their spouses, families and the community. In A. B. Cobb (Ed.), Medical and psychological aspects of disability. Springfield, IL: Charles C. Thomas.

Palmore, E.. \& Rivett, V. (1977). Change in life satisfaction: A longitudinal study of persons aged 46-70. Journal of Gerontology, 32, 311-316.

Pancoast, D. (1984). The contribution of social support to the successful functioning of men with epilepsy (Doctoral dissertation, Portland state University, 1984). Dissertation Abstracts International, 45, SECA, 1888 .

Pless, I. B., \& Satterwhite, B. (1973). A measure of family functioning and its applications. Social science and Medicine, I, 613-621.

Porrit, D. (1979). Sociai support in crisis: Quantity or quality. Social Science and Medicine, 13A, 715-721.

Rabkin J. G., \& Struening, E. L. (1976). Life events, stress, and illness. Science, 194, 1013-1020. 
Radloff, L. S. (1977). The CES-D Scale: A self-report depression scale for research in the general population. Applied Psychological Measurement, 1 , $385-401$.

Robertson, E. R., \& Suinn, R. M. (1968). The determination of rate of progress of stroke patients through empathy measures of patient and family. Journal of Psychosomatic Research, 12, 189-191.

Roberto, K. A., \& Scott, J. P. (1984). Friendship patterns among older women. International Journal of Aging and Human Development, 19, 1-10.

Robins, M., \& Baum, H. M. (1981). The National survey of Stroke. Incidence. Stroke, 12 (Part 2, Supp1. 1), I45 to I-57.

Robinson, R. G. (1986, April 15). Post-stroke mood disorders. Hospital Practice, pp. 83-89.

Robinson, R. G., Bolduc, P. L., Rubos, K. L., Starr, I. B., \& Price, T. R. (1985). Social functioning assessment in stroke patients. Archives of Physical Medicine and Rehabilitation, 66, 496-500.

Robinson, R. G. \& Szetela, B. (1981). Mood change following left hemispheric brain injury. Annals of Neurology, 9, 447-453.

Rook, K. S. (1984). The negative side of social interaction: Impact on psychological well-being. Journal of Personality and Social Psychology, 46 , 1097-1108.

Rosenberg, M. (1965). Society and the adolescent selfimage. Princeton, NJ: Princeton University Press.

Rotter, J. B. (1966). Generalized expectancies for internal versus external control of reinforcement. Psychological Monographs, 80 (Serial No. 609).

Rubenstein, E. , \& Federman, D. D. (Eds.). 1982. Scientific American medicine. New York: Scientic American, Inc.

Sacco, R. L., Wolf, P. A., Kannel, W. B., \& McNamara, P. M. (1982). Survival and recurrence following stroke: The Framingham Study. Stroke, 13, 290-295. 
Sandler, I. N., \& Barrera, M. Jr. (1984). Toward a multimethod approach to assessing the effects of social support. American Journal of Community Psychology, $\underline{12}, 37-52$.

Schaefer, C., Coyne, J. C., \& Lazarus, R. S. (1981). The health-related functions of social support. Journal of Behavioral Medicine, 4, 381-407.

Scheier, M. F.. \& Carver, C. S. (1983). Optimism: Assessment and implications of chronic outcome expectancies. Unpublished manuscript, Carnegie-Mellon University, Pittsburgh, PA.

Schulz, R., \& Decker, S. (1985). Long-term adjustment to physical disability: The role of social support, perceived control, and self-blame. Journal of Personality and Social Psychology, 48, $1162-1172$.

Schulz, R., \& Decker, S. (1982, November). Social support and well-being in middle-aged and elderly spinal cord injured persons: A social psychological analysis. Final Report submitted to the NRTA/AARP Andrus Foundation.

Schulz, R., \& Rau, M. T. (1985). Social support through the life course. In S. Cohen \& S. L. Syme (Eds.), Social support and health. New York: Academic Press.

Sexton, D. L. (1984). Wives of COPD patients. Cast in the role of caretaker. Connecticut Medicine, 48, 37-40.

Shanas, E. (1979). The family as a social support system in old age. The Gerontologist, 19, 169-174.

Shumaker, S. A., \& Brownell, A. (1984). Toward a theory of social support: Closing conceptual gaps. Journal of Social Issues, $40(4), 11-36$.

Silliman, R. A. (1984, March). Family careqivers and their frail elderly. Paper presented at the annual meeting of the American Geriatric Society, Denver, Colorado.

Silver, R. L.. \& Kortman, C. B. (1980). Coping with undesirable life events. In J. Garber \& M. E. P. Seligman (Eds.), Human Helplessness. New York: Academic Press. 
Soltero, I., Liu, R., Cooper, R., Stamler, J., \& Garside, D. (1978). Trends in mortality from cerebrovascular disease in the United States, 1960-1975. Stroke, 9 , 549-555.

Stachnik, T., Stoffelmayr, B., \& Hoppe, R. B. (1983). Prevention, behavior change, and chronic disease. In T. G. Burish \& L. A. Bradley (Eds.), Coping with chronic disease. New York: Academic Press.

Stallones, R. A., Dyken, M. L., Fang, H. C. H., Heyman, A., Seltser, R., \& Stamler, J. (1972). Report of the Joint Committee for Stroke Facilities I. Epidemiology for stroke facilities planning. Stroke, $\underline{3}, 360-371$.

Stein, R. W., Hier, D. B., \& Caplan, L. R. (1985). Cognitive and behavioral deficits after right hemisphere stroke. Current Concepts of Cerebrovascular Disease - Stroke, 20, 1-5.

Strain, J. (1979). Psychological reactions to chronic medical illness. Psychiatric Quarterly, 51, 173-183.

Strauss, A. L. \& Glaser, B. G. (1975). Chronic illness and the quality of life. St. Louis: c. V. Mosby.

Stueve, A., \& Fischer, C. S. (1978, September). Social networks and older women. Working Paper 292. Prepared for the Workshop on Older Women, Washington, DC. National Institute on Aging, National Institutes of Health.

Thoits, P. A. (1982). Conceptual, methodological, and theoretical problems in studying social support as a buffer against life stress. Journal of Health and social Behavior, 23, 145-159.

Toennies, F. (1957). Community and Society (C. P. Loomis, Trans.). East Lansing, MI: Michigan state University Press. (Original work published 1887).

Tolsdorf, C. (1976). Social networks, support, and coping: An exploratory study. Family Process, 15, 407-417.

Trudel, L., Fabia, J., \& Bouchard, J. P. (1984). Quality of life in 50 carotid endarterectomy survivors: A longterm follow-up study. Archives of Physical Medicine and Rehabilitation, 65, 310-312. 
Turner, R. J., Frankel, B. G., \& Levin, D. M. (1983). Social support: Conceptualization, measurement, and impiications for mental health. Research in Community and Mental Health, 3, 67-111.

Unks, R. P. (1983). The relative influence of social, physical, and psychological factors on the morale and life satisfaction of elderly wives of stroke patients: A descriptive exploratory study. (Doctoral dissertation, University of Washington, 1983). Dissertation Abstracts International, 44, SECA, 2585.

U. S. Bureau of the Census. (1983a). 1980 census of the population: Characteristics of the population, United States summary. (Dept. of Commerce Publication No. PC80-1-C1). Washington, DC: U.S. Government Printing office.

U. S. Bureau of the Census. (1983b). 1980 census of the population: General population characteristics, Oregon. (Dept. of Commerce Publication No. PC80 -1B39). Washington, DC: U.S. Government Printing Office.

Vaux, A. (1985). Variations in social support associated with gender, ethnicity, and age. Journal of Social Issues, 41 (1), 89-110.

Veroff, J., Douvan, E., \& Rulka, R. (1981). The inner American. New York: Basic Books.

Vinokur, A.. \& Selzer, M. L. (1975). Desirable versus undesirable life events: Their relationship to stress and mental distress. Journal of Personality and Social Psychology, $32,329-337$.

Walker, R. N., MacBride, A., \& Vachon, M. L. S. (1977). Social support networks and the crisis of bereavement. Social Science and Medicine, 11, 35-41.

Wallston, B. L., Alagna, S. W., DeVellis, B. M. \& DeVellis, R. F. (1983). Social support and physical health. Health Psychology, 2 , 367-391.

Ward, R. A., Sherman, S. R. \& Lagory, M. (1984). Subjective network assessments and subjective wellbeing. Journal of Gerontology, 39, 93-101.

Webster, E. J. (1980). Home and family: Problems and payoffs. In R. Brookshire (Ed.), Clinical Aphasiology Conference Proceedings, 1980. Minneapolis, MN: BRK Publishers. 
Webster, E. J.. \& Newhoff, M. (1981). Intervention with families of communicatively impaired adults. In $D . S$. Beasley \& G. A. Davis (Eds.), Agina: Communication processes and disorders. New York: Grune and stratton.

Weinfeld, F. D. (Ed.) (1981). The National Survey of stroke. Stroke, 12 (Part 2, Suppl. 1).

Weiss, R. S. (1974). The provisions of social relationships. In $\mathrm{Z}$. Rubin (Ed.), Doing unto others. Englewood Cliffs, NJ: Prentice-Hall.

Wellman, B. (1981). Applying network analysis to the study of support. In B. H. Gottlieb (Ed.), Social networks and social support. Beverly Hills, CA: Sage Publications.

Wellman, B. (1985). From social support to social network. In I. G. Sarason \& B. R. Sarason (Eds.), Social support: Theory, research and applications. Dordrecht, The Netherlands: Martinus Nijhoff.

Wentowski, G. J. (1981). Reciprocity and the coping strategies of older people: Cultural dimensions of network building. The Gerontologist, 21, 600-609.

Wilcox, B. I. (1981), Social support, life stress, and psychological adjustment: A test of the buffering hypothesis. American Journal of Community Psychology. g. 371-386.

Williams, A. R. (1985). Physical illness and depression: Changes over time in middle aged and elderly persons (Doctoral dissertation, Portland state University, 1985). Dissertation Abstracts International,

Wirsching, M., Druner, H. U., \& Herrmann, G. (1975). Results of psychosocial adjustment to long-term colostomy. Psychotherapy and Psychosomatics. 26, 245256 .

Wirth, L. (1938). Urbanism as a way of life. American Journal of Sociology, 44, 3-24.

Wood, V., \& Robertson, J. F. (1978). Friendship and kinship interaction: Differential effect on the morale of the elderly. Journal of Marriage and the Family, 40, 367-375. 
Wortman, C. B. (1984). Social support and the cancer patient. Conceptual and methodologic issues. Cancer, 53 (10) (Suppl.), 2339-2362.

Wortman, C. B., \& Conway, T. L. (1985). The role of social support in adaptation and recovery from illness. In S. Cohen \& S. L. Syme (Eds.), social support and health. New York: Academic Press.

Wortman, C. B., \& Dunkel-Schetter, C.. (1979). Interpersonal relationships and cancer: A theoretical analysis. Journal of Social Issues, 35 (1), 120-155.

Wylie, C. M. (1967). Measuring end results of rehabilitation of patients with stroke. Public Health Reports, 82 (16), 893-898.

Zarit, S. H., Reever, R. E. \& \& Bach-Peterson, J. (1980). Relatives of the impaired elderly: Correlates of feelings of burden. The Gerontologist, 20, 649-655.

Zarit, S. H., Todd, P. A., \& Zarit, J. M. (in press). Subjective burden of husbands and wives as caregivers. The Gerontologist.

Zung, w. W. R., \& Durham, N. C. (1965). A self-rating depression scale. Archives of General Psychiatry, 12, 63-70. 
APPENDIX A

INITIAL INTERVIEW SCHEDULES 
STROKE SURVEY: SOCIAL SUPPORT AND WELL-BEING

INITIAL INTERVIEW SCHEDULE:

PRIMARY INFORMANT

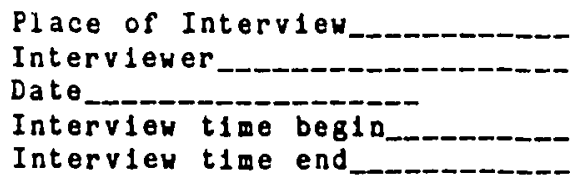

Dyad I.D.

Interviewer

Intervieu time begin

Respondent Code

$1=P I$

$2=S V$

Interviey time end

$3=P I$ re: SV

First, let we thank you for taking the time to talk with us. We are very interested in learning more about stroke patients and their famliles and frlends. I have a number of questions to ask you, so let we know if you need to rest, to take a break, or want further explanation. Let me reassure you that all information you provide will be kept confidential.

The first set of questions will cover general background Inf ormation.

PI-SV RELATIONSHIP:

1. What is your relationship to $?$

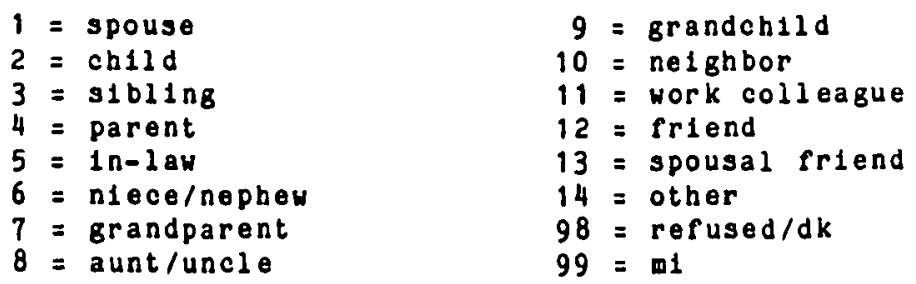

2. How long have you known

$?$

\section{DEMOG RAPHICS :}

3. What is your date of birth?

4. SeX (INTERVIEHER CODE)

$1=$ female
$2=$ male

5. What is your ethnic background? (ASK ONLY IF NOT OBVIOUS)

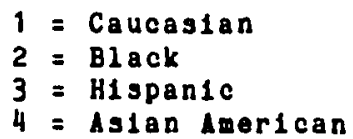

$5=$ Mmerican Indian

6 = other

$B=$ refused/dk

$9=m 1$ 
6. What is your marital status? (HAND CARD TO R)

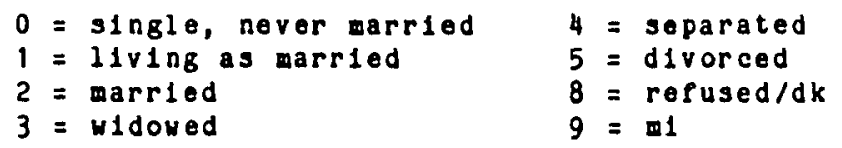

7. How long have you been years/montbs

8. Do you have any chlldren, stepchildren, or adopted children? $0=$ no

Total of children:

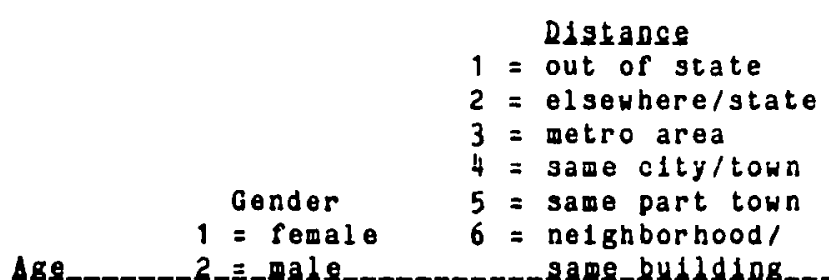

$1=$ remale 6 = nol ghbor hoodl
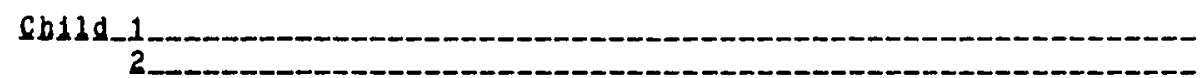

3

4

5

(Use other side if > 5 children.) 
9. Makeup of Household:

I'd like to ask some questions about the makeup of your household, about the people who live with you (and _-_._. ). What are the first names of all the other persons who live with you on a regular basis? (LIST BELOW)

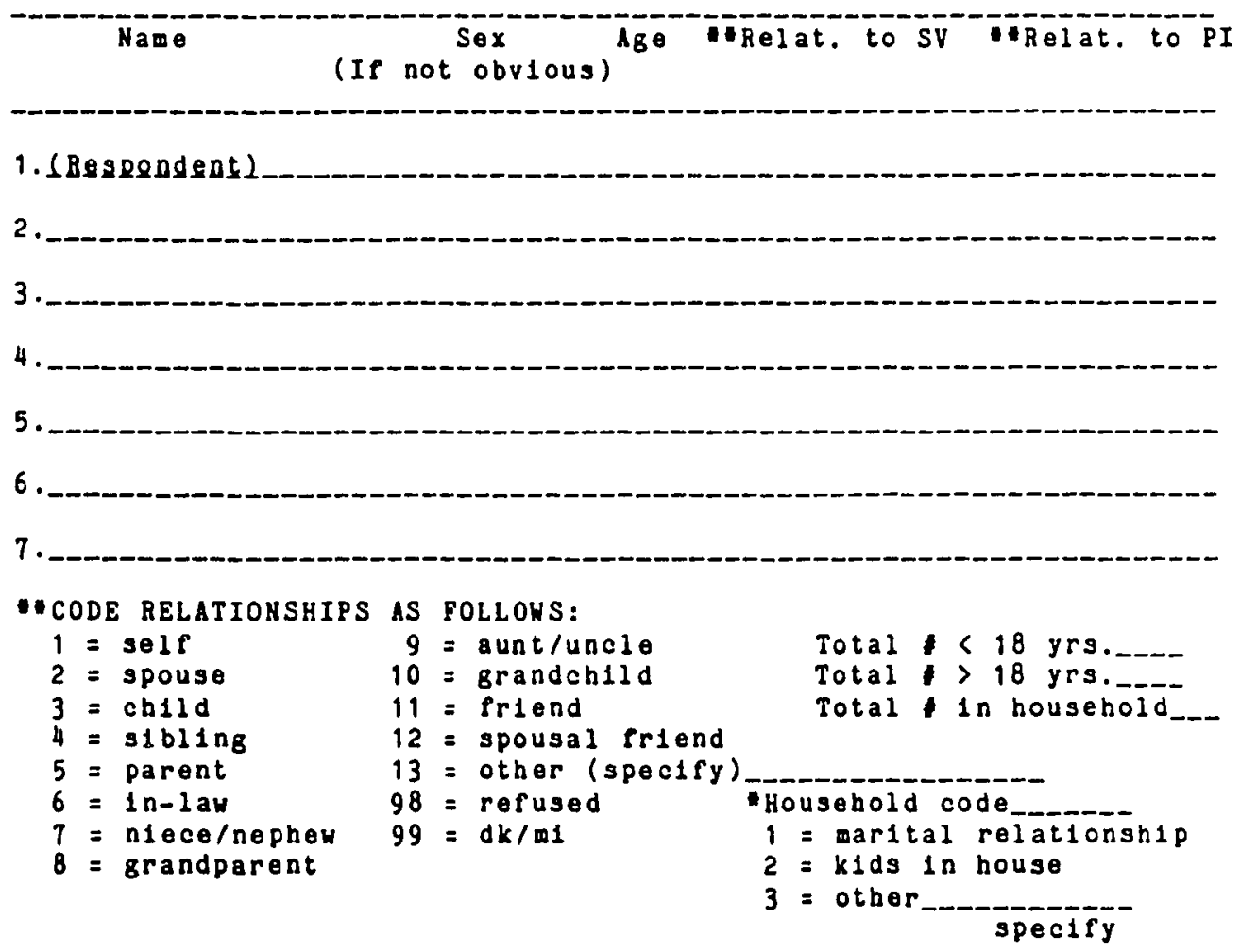

10. What is the highest level of education you have completed?

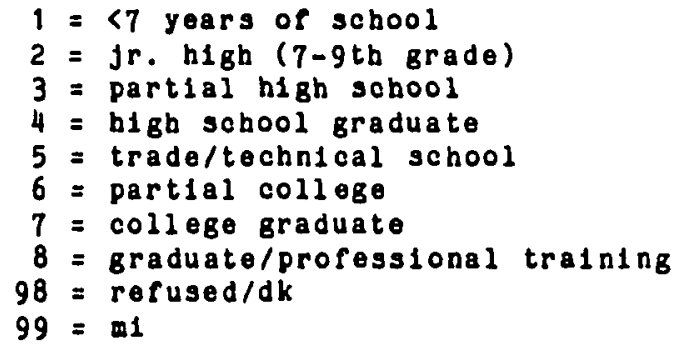



history.

Now, I'd like to ask you some questions about your employment

11. At the time of the stroke, what was your employment status? ( $H A N D$ CARD TO R)

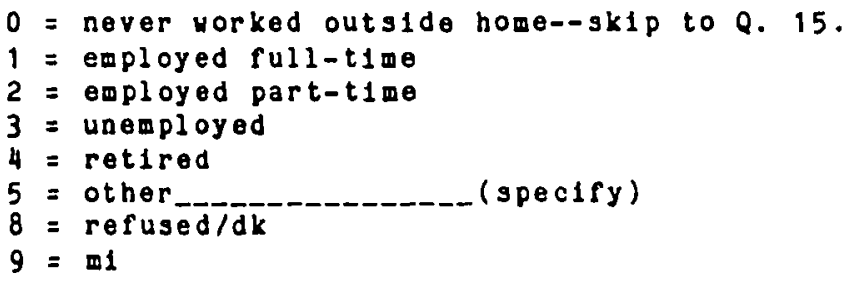

12. How long have you been (were you) $?$

- - - - - years (months)

13. What 1s (was) your occupation called?

(PROBE: self employed/own business)

EOB_ANSHEB_1__2:

14. In general, how satisfled are you (have you been) with being a $?$ (HAND CARD TO R)

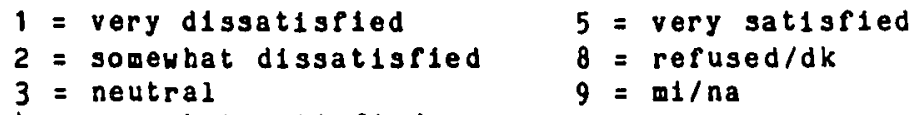

15. In which of the following categorles did your last year's total beysebeld income fall? (HAND CARD TO R)

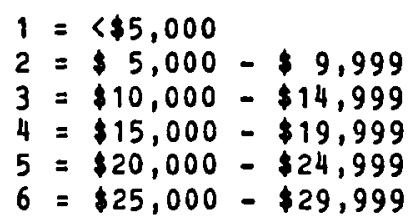

The next set of questions will be asking you about your ifing arrangements prior to the stroke.

16. How long have you lived at your current residence? -__-__nears (months)

17. Residence setting? (INTERVIEWER CODE)
1 = urban
$9=m 1$
2 = rural
3 = suburban 
18. In what type of residence do you I1ve? (INTERVIEHER CODE)
1 = slngle ramlly
$2=$ duplex
3 = apartment
$4=$ condo
5 = subsidized housing

19. Do you own or rent your residence?
$1=$ own
$8=$ refused $/ d k$
$2=r e n t$
$9=m$

20. How long have you lived in the area?

-

21. What forms of transportation do you ut1lize; how do you run errands, visit friends and relatives, etc.? (CIRCLE ALL THAT APPLY)
1 = publ1c
2 = friends
$3=$ relatives
$4=\operatorname{tax} 1$

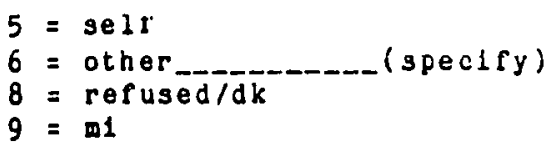

22. Do you bave any pets in your residence? (CIRCLE ALL THAT APPLY)

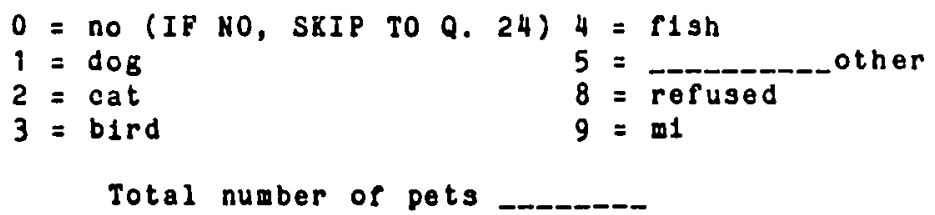

23. How attachod to your pet(s) do you consider yoursely? (HAND CARD TO R)

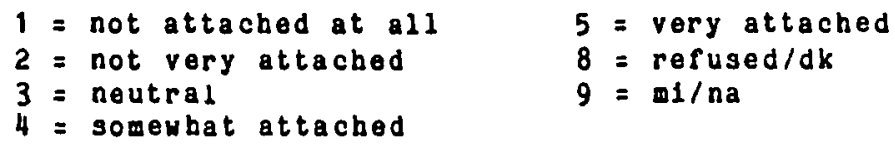

ORGANIZATIONAL INVOLVEMENT/ACTIVITIES:

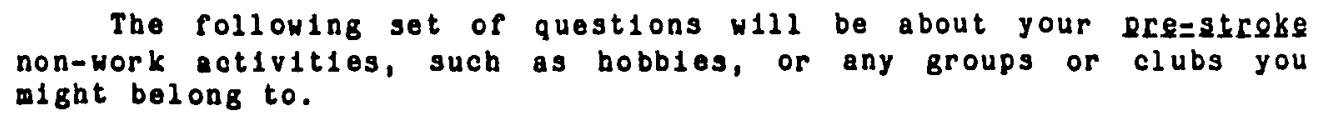


24. Hom important is rel1gion In your l1So? (HAND CARD TO R)

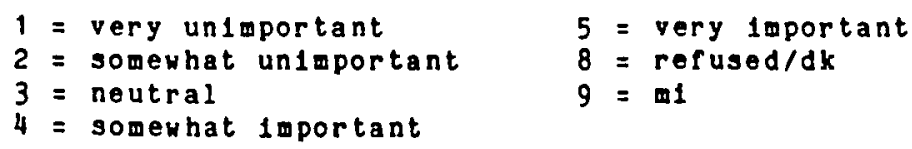

25. What is your rel1810us prérerence?

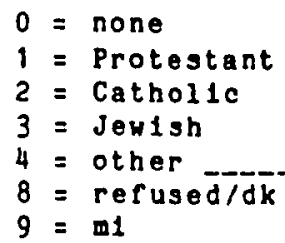

26. Before the stroke, how active were you in your religious organization? (READ RESPONSES)

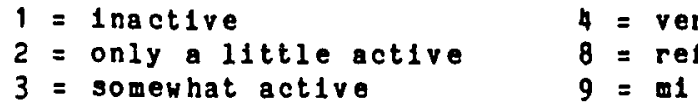

27. How often did you attend religlous services?

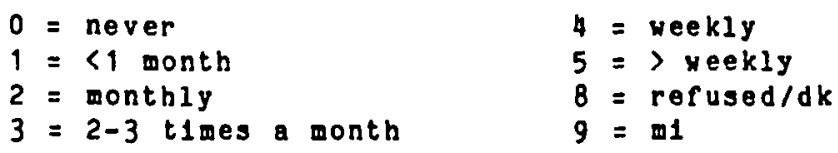


28. The next set of questions w111 be about your membership in clubs, groups, or organizations. Flrst, can you tell we what organizations you presently belong to? (LIST BELOW) Now I bave a few questions to ask about your membership in each of these organizations.

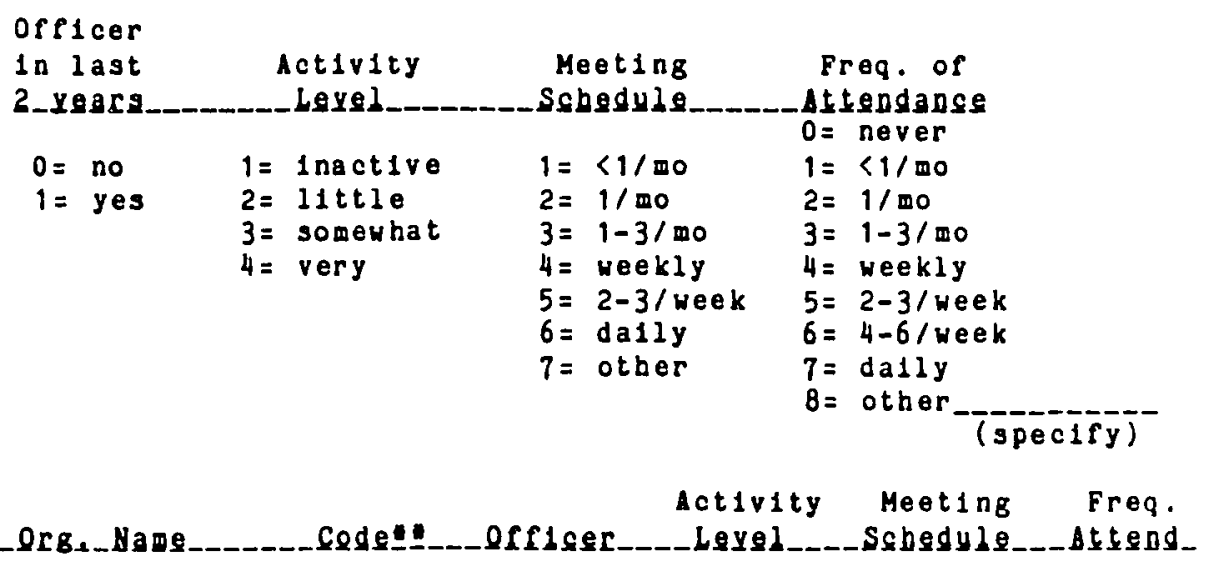

\section{- ORGANIZATIOH CODE}
$0=$ none
1 = business, prof or tech
2 = church connected
$3=\operatorname{char} 1 \operatorname{tablo}$
$4=$ c1v1c, community or ne1ghborhood $10=$ other
5 = eraternal or veterans $\quad 99=m 1$
$6=$ political

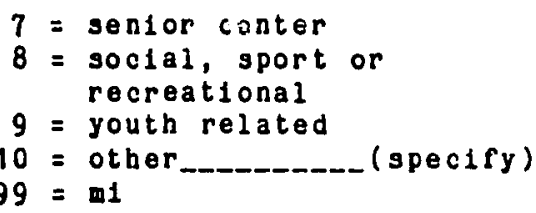

7 = senior conter

$8=$ social, sport or recreational

10 other
$9=\mathrm{m} 1$ $-(\operatorname{specify})$ 
29. Do you give your time to other volunteer work?

$0=$ no--1f no, sk1p to Q. 30

$1=$ yes

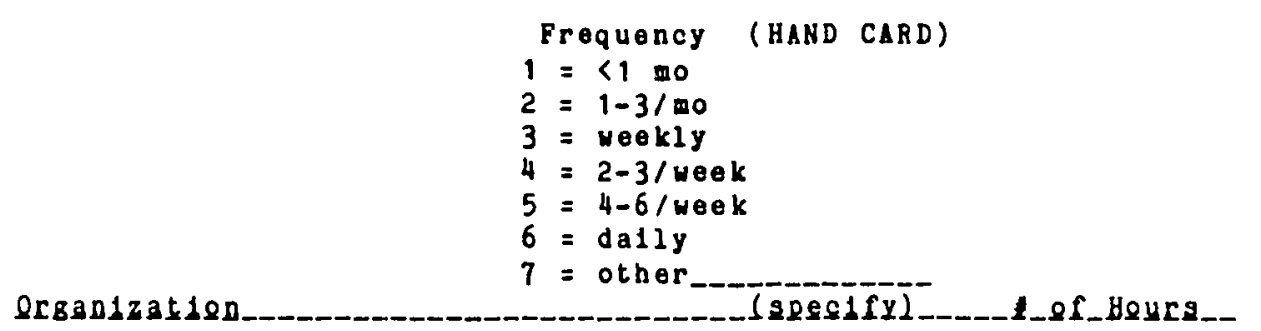

30. Do you bave any special interests, pursuits, or hobbles? (PROBE: What do you do witb your spare time?)

$$
0=N_{0}, \text { notbing }
$$

List - Besponse

\section{Erequency (HAND CARD)}

$1=\langle 1$ mo

$2=1-3 / \infty 0$

$3=$ weekly

$4=2-3 /$ week

$5=4-6 /$ week

$6=d a i l y$

$7=$ other (gResify) 
Code

31. Have you used community services and/or agencies in the past year? ( $P$ ROBE)

Frequency
(see above for
Seryise_Name_responge_set)

- SERVICE CODE

$0=$ none, no

1 = Meals on Wheels

$2=\mathrm{VNA}$

$3=\mathrm{Sr} . \operatorname{Ctr}$. (Loaves/Ftshes)

4 = Food Stamps

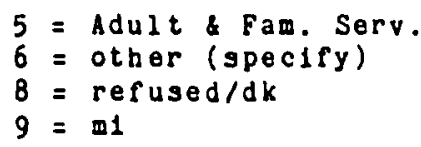

Total of Services

Sum of Frequency.

HEALTH/WELL-BEIHG :

In the following sections are questions regarding your health and well-being.

32. Were there any 1oportant good or bad events that happened to you in the year before the stroke? (FOR EXAMPLE: Death of someone close, birth of grandchild, retirement, serlous accident, etc.)

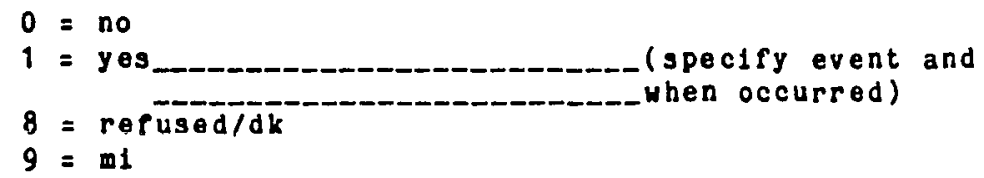

33. Did you bave any physical disabilities that limited your work or social activities before the stroke?

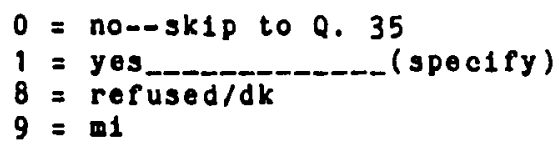

34. Does th1s _._._. require the uge of special services, alds or equipment? (PROBE: For example, braces, vision or hearing aids, band rails, bospltal beds, etc.)

$$
\begin{array}{ll}
0=\text { no--1f no, probe } & 8=\text { refused } / \mathrm{dk} \\
1=\text { yes_n_ } & 9=\mathrm{m} 1 / \mathrm{na}
\end{array}
$$


35. How would you rate your overall bealtb at the present time? (HAND CARD TO R)

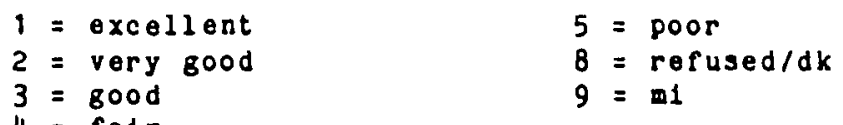

36. Is your bealth now better, about the same or worse than it was six wonths ago?

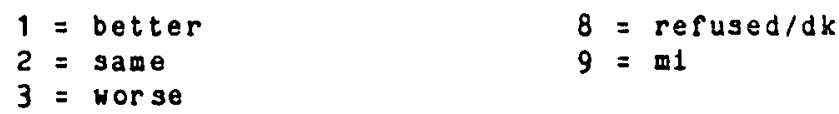

37. Do you feel that within the next six months your overall health is likely to (READ RESPONSES)

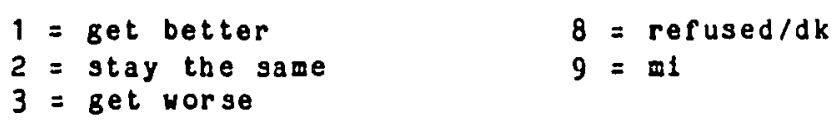

38. Do you have any chronic health problems? (IF NO ANSWER, PROBE: e.8., Diabetes, heart condition, bigh blood pressure)

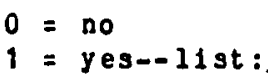

39. Have you been bospltalized in the one year before the stroke?

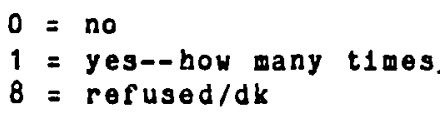

lengtb of hospitalization (days) for each - - $9=n a$

40. In the six months prior to the stroke, how many times have you visited the doctor?

Total (if 0 , ask Q. 41)

41. When was the last time you saw a doctor? 
Intilal Intervien

Code

42. Prior to the stroke, what medications were you taking on a regular basis?
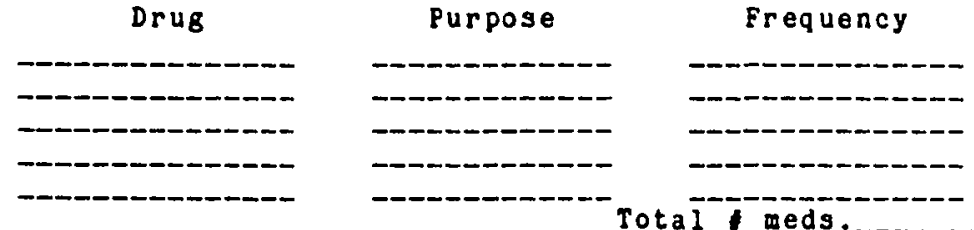

43. Are you taking any medicines now that you were not taking prior to the stroke?

\begin{tabular}{ccc} 
Drug & Purpose & Frequency \\
\hdashline
\end{tabular}

44. In the six months before the stroke, did you have an emotional problem for which you wanted help?

$$
\begin{aligned}
& 0=\text { no } \quad 8=\text { refused } / \mathrm{dk} \\
& 1=\text { yes (IF YES, ASR Q. 45) } 9=\text { m1 }
\end{aligned}
$$

45. Did you recelve help for this concern?

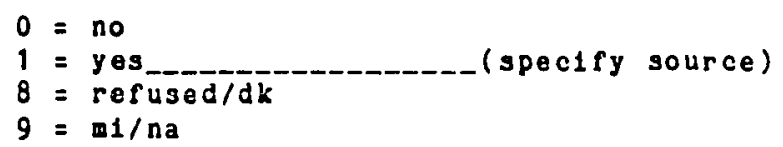

46. In the six months before the stroke, which of the following choices best describes your appetite? (HAND CARD TO R)

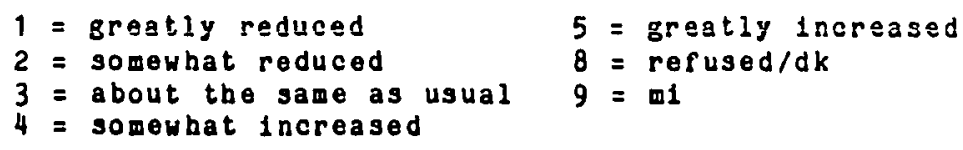

47. Did you experience any change in your sieeping habits in the six months before the stroke?

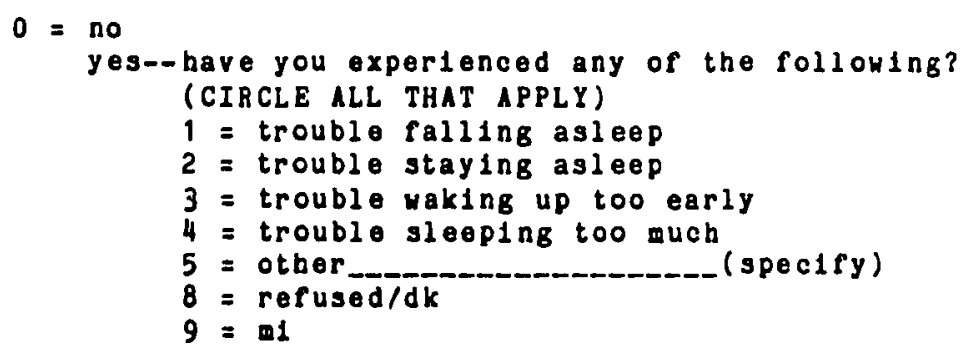


Intial Interview

53. Up to now, bow satigfled are you with the health care received for the stroke? (HAND CARD TO $R$ )

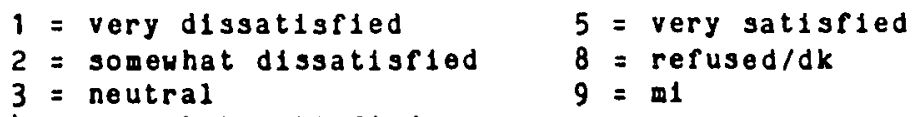

54. Have you known anyone else who has bad a stroke?

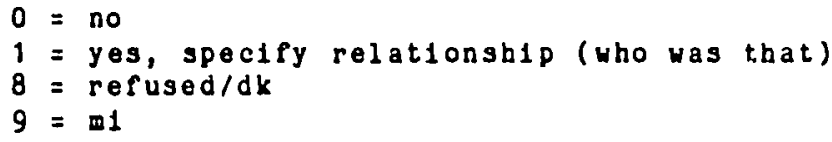

55. Who or what do you blame mest for the stroke happening?

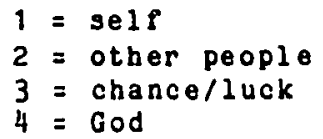

(Q. 56-67 TO BE ASKED ONLY IF SY/PI HAVE SPOUSAL/MARITAL RELATIONSHIP)

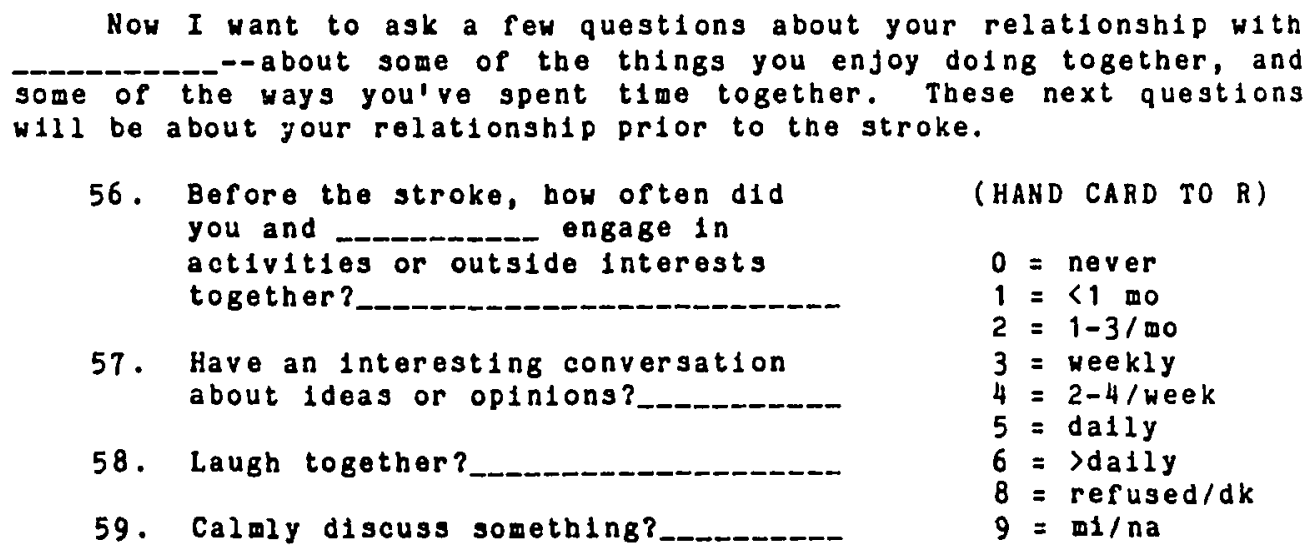

60. Work together on a project? 


Initial Interview
61 . Before the stroke, did you and
the same organizations? (SPECIFY NAME AND CODE BY TYPE OF
ORGAIZAIION):
Name of
Organization Type or

- organization code

$0=$ none

1 = business, prof or tech

- of organizat.

2 = church connected

- X requency

$3=\operatorname{charitable}$

$4=$ civ1c, community or nelghborhood

5 = fraternal or veterans

$6=$ political

7 = sentor center

$8=$ social, sport or recreational

$9=$ youth related

$10=$ other (specify)

$98=$ refused $/ d k$

$99=\mathrm{ml} / \mathrm{na}$

62. In describing your relationship with over the years, please tell me how often the two of you would be likely to disagree on the follouing:

handling (family) finances

ways of dealing with oh1ldren, In-laws, or relatives

$1=$ rarely/almost never disagree

maklng ajor dectsions

2 = scmetimes disagree

household tasks

3 = freq. disagree

bousehold tasks

$8=$ refused $/ d k$

lelsure time interests or

$g=m 1 / n a$ activities. 
63. In your famlly, who bas beld the primary responsibility for:

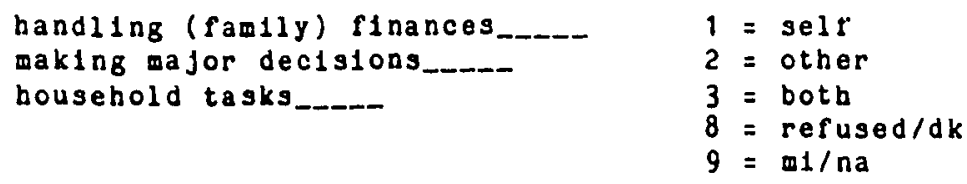

64. Think about your relationsbip with _...... Before the stroke, if you had a very personal and serlous problem, how likely would you be to discuss it with (HAND CARD TO R)

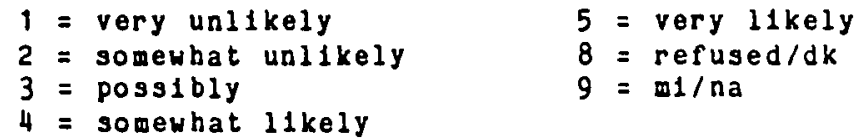

65. Aga1n, before the stroke, if personal and serlous problem, how $11 \mathrm{kely}$ would he/she be to discuss it with you? Would you say it was (HAND CARD TO R)

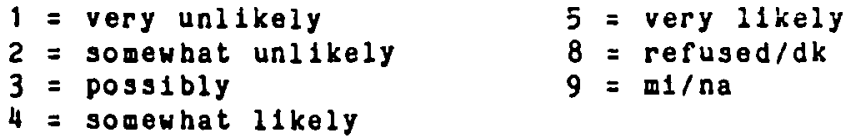

66. In general, how happy are you with your marriage (relationship)? (HAND CABD TO R)

1 = yery unhappy

2 = somewhat unhappy

3 = neutral

4 = somewhat happy

67. Compared to other couples you have known, overall, would you rate your marriage (relationship) as worse, about the same as, or better than other couples?

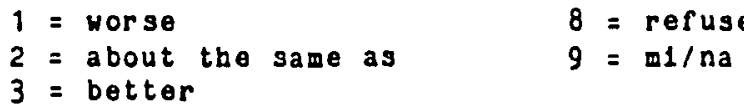

SOCIAL NETWORR-SOCIAL SUPPORT DATA: BEFORE THE STROKE:

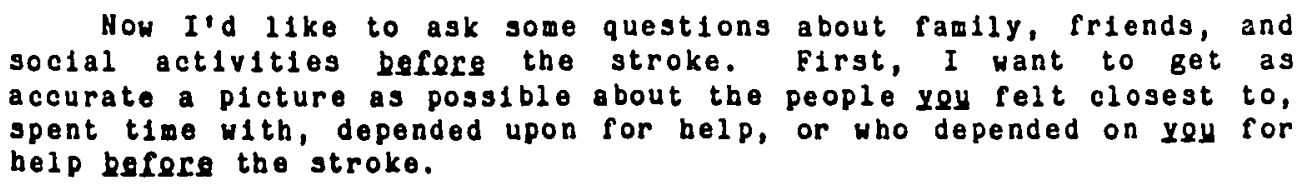


68. In the past six months, about how often have you:

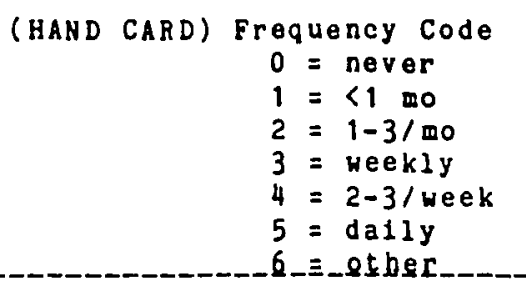

(1) Had someone to your home for lunch or dinner

(2) Gone to someone's home for lunch or dinner

(3) Had someone come by your house to visit

(4) Gone over to someone's house for a visit

(5) Gone out with someone (to a restaurant, movie, etc.) -

69. Sometimes friends, nelghbors, or family members perform helpful tasks for each other, such as watching the house when one is out of town, plcking up the mall, helping with olnor hougehold repalrs or chores, etc. Are there people who would help you in these way 3 ?

$$
\begin{aligned}
& 0=\text { no } \\
& 1=\text { yes }
\end{aligned}
$$

(IF YES, ASK FOR FIRST MAMES AND RECORD ON NAME LIST I. FOLLOW SAME FORMAT AND RECORD NAMES FOR Q.'S 70-83.) ways?

70. Are there people who would ask yol to help them in these

71. Thinking back to the six months before the stroke, do you have any friends, nelghbors, or family members who would help you if you were sick for a short time? That 1s, for a few days up to a week?

72. How about people who would ask you to help then if they were sick for a short time? Do you have any friends, neighbors, or famlly members who would ask for your help in this way?

73. When you are concerned about a personal matter, for example, about someone you are close to, or something you are worried about, how often do you talk about it with someone? Would you say, 
74. Think about the last six months, especlaliy the time before the stroke, when you did talk with someone about personal matters, whom did you talk with?

75. Are there people who would come to you to talk about something they were worried about, or about a personal matter? Remember, it can be a family member, friend, or nelghbor.

76. Often, people ask advice, or rely on the judgment of someone they know in making important decisions about their lives--for example, about their family or thelr work. Is there anyone whose opinion, in the six months before the stroke, you considered seriously in making important decisions? May I have their first names?

77. Are there people who would seek your opinion or advice about things?

78. Sometimes people get together with close friends, neighbors, family or others to do something enjoyable together, or just to socialize. May I bave the first names of the persons you've done (or do) these things with op_a_regular_bagls?

79. Sometimes people get together with others to talk about hobbles or spare-time interests they have in common. Do you ever do this? H1th whom?

80. If, in the time before 's stroke, you had been 111 , or needed help for longer than just a rew days, say weeks or months, is there someone, or more than one person, you could call on to help, come and stay ytth you if needed, or take you into the1r home?

81. Sometimes, people bave one person, or a few people they feel closest to--people they can confide in, or share personal worries and concerns with. Who are the people you feel closest to in this way?

82. (IF SPOUSE IS NOT NAMED, ASK ABOUT SPOUSE) WOuld you say that you feel closer to this person (these people) than your spouse?

\section{3. (PRESENT NAME LIST I)}

Are there any other people who were important to you before the stroke whose names are not on th1s 11 st? For example, friends, relatives, ne1ghbors, co-workers, or members of the same organization? 
(ADD ANY ADDITIONAL NAMES TO NAHE LIST I)

SOCIAL NETWORK DATA: PRIMARY INFORMANT'S SOCIAL SUPPORT TIME OF STROKE:

Now, I want to ask you about the situation as it's been these past few weeks, or slnce _-____-'s stroke. I'd like to find out a little about the people and kinds of support that bave been helpful to you.

88. If you needed a ride to the bospital to visit are there people you could call on to help?

$$
\begin{aligned}
& 0=\text { no } \\
& 1=\text { yes }
\end{aligned}
$$

Could you give we the first names of the people you would ask? (RECORD NAMES ON NAME LIST II. USE SAME FORMAT FOR Q.'S 89-99.)

89. Have there been some people who've actually helped in this way, that 1s, provided transportation help during 11 iness?

90. If you needed someone to keep an eye on the house, take in a package, or prepare a meal while you spent time at the hospital, are there people you would ask to belp in this way?

91. Have there been people who actually helped in this yay since 's 1liness, that 1s, kept an eye on the house or helped with some chores?

92. Are there people who've been to visit you, to offer you "moral support"?

93. About how many people bave called to find out how and you were dolng? (PROBE: Would you say more or fewer than 15?)

94. Are there people who've been helpful in other ways through tbis time of 111 ness, for example, who've come to stay with you or had you stay with them, or offered to run errands for you? 
95. Sometimes friends, nolghors, and famlly members can be helpful in providing information/advice when we need 1t. Are there any of these people who've given you belpful information or advice in the last few weeks about stroke, ways to deal with a stroke, or about resources to belp stroke patients and thelr fam1lies?

96. Are there any people you know who appreclate or understand what you are going througb right now as the _._._._. of a stroke patient?

97. Are there any other ways in which people you feel close to have helped, or been there to provide support since stroke? Who has provided this belp?

98. Sometimes, in these c1rcumstances, we rind that there are people who help more than we expect them to, or people who are helprul when we didn't expect them to help at all. Are there people who have belped more than you expected, or who have helped when you didn't expect them to help at all?

$$
\begin{aligned}
& \text { Helped/didn't expect } \\
& \text { or more than expected. }
\end{aligned}
$$

\section{9. (PRESENT NAME LIST II)}

Are there any other people who were important to you during this time of the stroke whose names are not on this 11st? For example, friends, relatives, nelghbors, co-workers, or members of the same organization?

(ADD AHY ADDITIOHAL MAMES TO NAME LIST II)

SOCIAL RETHORE DATA - NEGATIVE ASPECTS:

In situations like this, sometines we find that there are people who don't help as much as we expect them to, or who don't help at all when we expected them to. 
Intial Interviou

20

Code

101. Are thore people who have belped during this time of 111 ness, but not as wuch as you expected they would, or who haven't helped at all when you thought they would?

Hay I bave thoir pirst names? (RECORD NAMES AND OTHER INFORHATION BELOW)

Relationship Helped less
Name

- Legs help than expected

or no help when expected

102. We all know people who try to help, but end up waklng the situation worse. Are there any people you reel have been a hinarance to _-____- 's recovery thus far eyen though they try to be helpful?

$0=$ no

$1=$ yes

What are their names? (RECORD NAMES AND OTHER INFO BELOW)

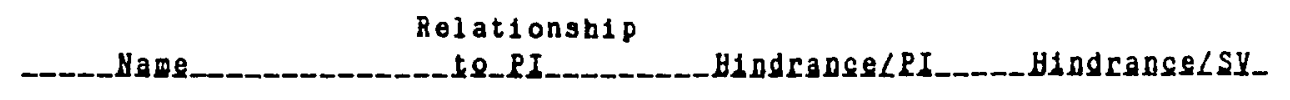

- Hindrance 
103. Sometimes we run 1 nto people who seem to be out to make life difficult. Are there people lixe that who have made problems for you?

$$
\begin{aligned}
& 0=\text { no } \\
& 1=\text { yes }
\end{aligned}
$$

Who would they be? (RECORD NAMES, RELATIONSHIPS BELOH)

104. We all know people we find it easy to get along with, and others we don't get along with. Are there people you don't get along with?

$$
\begin{aligned}
& 0=\text { no } \\
& 1=\text { yes }
\end{aligned}
$$

May I bave their first names? (RECORD NAMES AND RELATIONSHIPS BELOW) Name Belat100sh1D_to_PI 
Code

INSTRUCTIONS FOR COMPLETING NAME LISTS, CODING SHEETS, AND MATRIX:

1. FOR NAME LIST I, RECORD INFORMATION REGARDING RELATIONSHIP, AGE, SEY, AND CLOSENESS ON NAHE LIST I FORM.

2. REPEAT FOR NAME LISTS II AND A.

3. ON CODING SHEET, RECORD NAMES OF UP TO 10 PERSONS IN APPROPRIATE BOXES ACROSS THE TOP, BASED UPON THE FOLLOWING CRITERIA :

a. THOSE WHOM RESPONDENT INDICATES THEY (OR SV) ARE ESPECIALLY CLOSE TO.

D. THOSE RESPONDENT INDICATES AS MOST IMPORTANT PROVIDERS OF SUPPORT, IF NOT ALREADY SELECTED.

4. IF 10 OR FEWER NW MEMBERS, ASK QUESTIONS ON CODING SHEET ABOUT ALL OF THOSE MAMED.

5. COMPLETE INFORMATION ON CODING SHEETS, USING APPROPRIATE SHEETS FOR PI AND SV.

6. CIRCLE haMes THAT aPPEAR ON BOTH SV AND PI CODING SHEETS.

7. COMPLETE INFORMATION ON NH MATRIX SHEET.

105. In general, how satisfied are you with the amount of social contact you baye with otbers? (HAND CARD TO R)

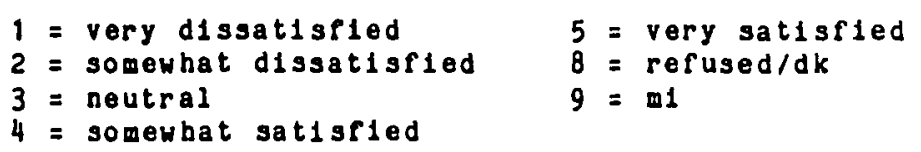

106. In general, how satisfled are you with the quality or closeness of the social contact you have with others? (HAND CARD TO R)

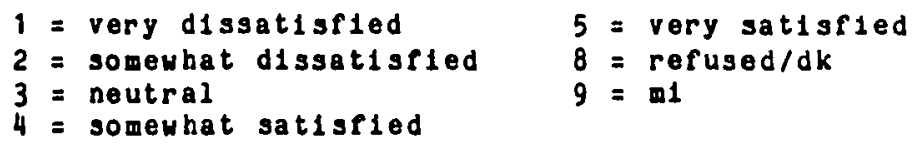


Inltial Interview

Code

107. As you can see, I've asked you many different kinds of questions about you and _....... But everyone is different and I might bave left out something that is important to you. Can you think of anything that I should have asked about you and _...... and the stroke but didn't? 
Intial Intervieu

Current Residence (SV only)

$1=$ hospltal

2 = rebabilitation facility

$3=$ nursing bome

$4=$ home

$5=$ other

$9=\sin \sin$

Respondent's degree of cooperation

$1=$ excellent

$2=800 d$

$3=f a 1 r$

$4=$ poor
24

Code

Did respondent mention

wanting summaryl

final report?

$0=$ no

$1=$ yes

Was anyone elso present during this interview?

$0=$ no

1 = yes--specify: approximate age of persons, sex and relationship to respondent.

How long was this person present?

D1d this person participate in interview responses?

How well do you feel the respondent urderatood the items of this Intervien?

1 = understood all itoms with no trouble

2 = understood most 1 tems, but had trouble with a few

3 = understood very few 1 tems, had trouble with most

4 = bad trouble understanding all items

Note below any other unusual problems in this interview 
STRORE SURYEY: SOCIAL SUPRORT AHD HELL- DEIAG

INITIAL INTERVIEH SCHEDULE:

STROKE VICTIH

Place of Interview

Intervieuer

Date

Interview time begin

Interview time end
Dyad I.D.

Respondent Code

$1=\mathrm{PI}$

$2=S V$

$3=P I r \theta: S V$

First, let we thank you for taking the time to talk with us. We are very linterested in learning more about stroke patients and their famllies and friends. I have a number of questions to ask you, so let me know if you need to rest, to take a break, or want further explanation. Let me reassure you that all information you provide will be kept confidential.

The first set of questions will cover general background information.

\section{DEMOGRAPHICS:}

3. What ig your date of birth?

4. SEX (INTERYIEWER CODE)

$1=$ female

$2=$ male

5. What is your ethnic background? (ASK ONLY IF NOT OBVIOUS)
1 = Caucasian
2 = Black
3 = Hispanic
4 = Asian American
5 = Amer1can Indian
6 = other
$8=\mathrm{refused} / \mathrm{dk}$
$9=m 1$

6. What is your marital status? (HAND CARD TO R)
$0=$ single, never married
$1=11 v \operatorname{lng}$ as married
4 = separated
$2=\operatorname{marr} 1 \mathrm{ed}$
$3=$ widowed
$5=d 1$ vorced
$8=$ refused $/ d k$
$9=m 1$

7. How long have you been $-?$ years/months 
8. Do you have any chlldren, stepchildren, or adopted children? $0=$ no

Total of children:

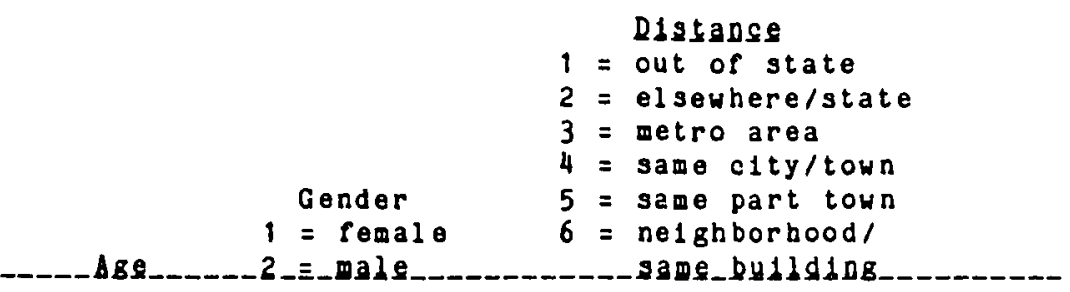

Cold 1

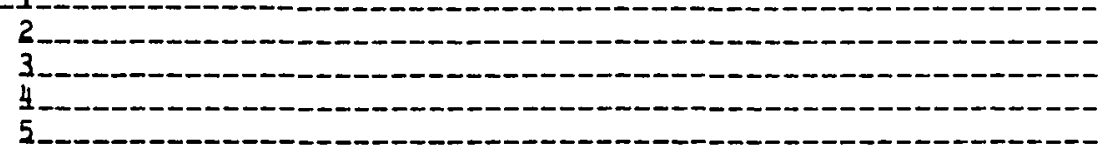

(USE OTHER SIDE IF > 5 CHILDREN)

9. Hakeup of Housebold:

I'd like to ask some questions about the makeup of your housebold, about the people who live with you. What are the first names of all the other persons who live with you on a regular basis? (LIST BELOH) Name

Sex Age

- Relat. to SV

\section{1. (Hespendent)} (If not obvious)

2.

3.

4.

5.

6.

7 .

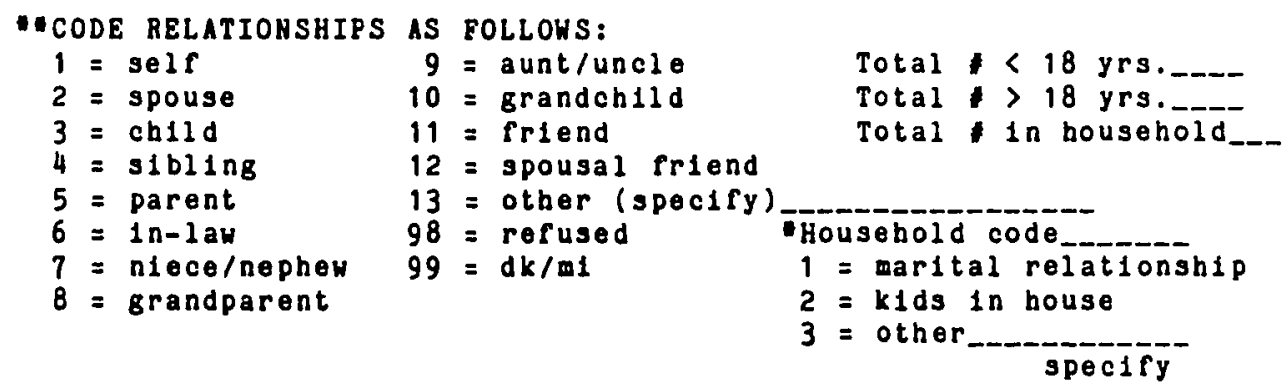


Code

10. What 1 s the bighest level of educstion you have coopleted?

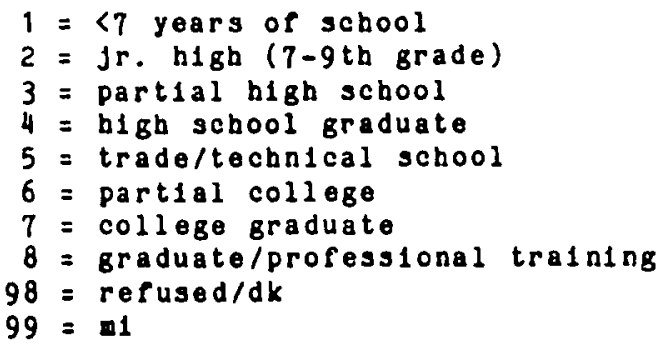

Now, I'd like to ask you some questions about your exployment

11. At the time of the stroke, what was your employment status? (HAND CARD TO R)

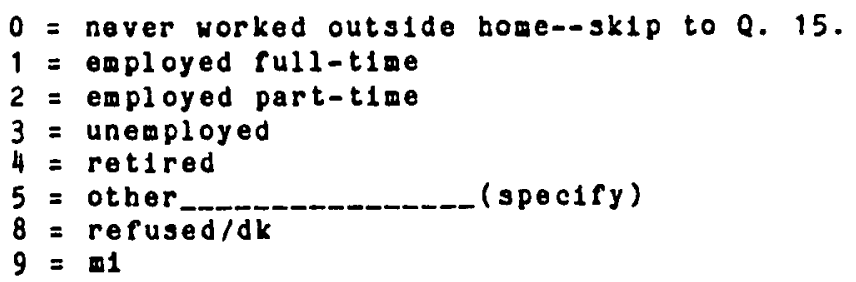

12. How long bave you been (were you) $-?$ years (zonths)

13. What is (was) your occupation called? (PROBE: self employed/own business)

EOB_ANSHEB_1__2 :

14. In Beneral, how satisfied are you (bave you been) with being a $?$ (HAHD CARD TO R)

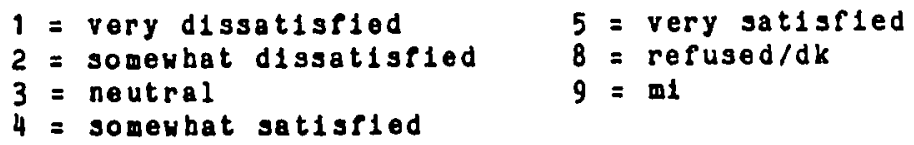

15. In which of tho following categories did your last year's total bousebeld income fall? (HAND CARD TO $R$ )

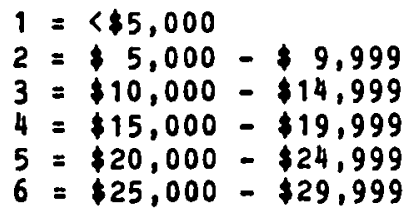

$7=\$ 30,000-\$ 39,999$

$8=\$ 40,000-\$ 49,999$

$9=>\$ 50,000$

$98=$ refused/dk

$99=m 1$ 
The next set of questions will be asking you about your living arrangements prior to the stroke. (ASK $16-20$ OF SV ONLY IF NOT ALREADY DETERMINED.)

16. How long have you lived at your current. residence?

- - - - - years (months)

17. Residence setting? (INTERVIEWER CODE)

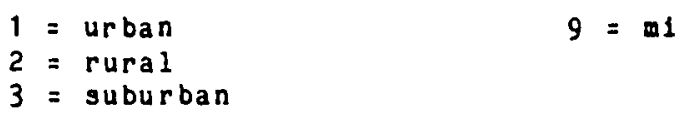

18. In what type of residence do (did) you live? (INTERVIEWER

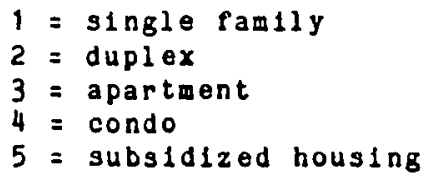

19. Do (d1d) you own or rent your residence?
$1=$ own
$8=$ refused/dk
$2=$ rent
$9=m 1$

20. How long bave you lived in the area? years (months)

21. What forms of transportation did you utilize; how did you run errands, visit friends and relatives, etc.? (CIRCLE ALL THAT APPLY)

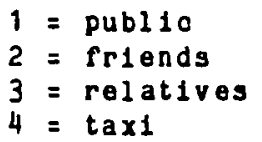

22. Do you have any pets in your residence? (CIRCLE ALL THAT APPLY)

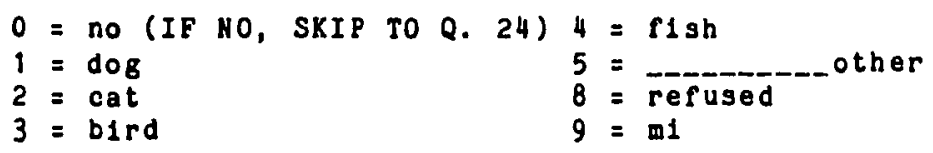

Total number of pets 
28. The next set of questions w11l be about your dembership in clubs, Broups, or organizations. First, can you tell me what organizations you presently belong to? (LIST BELOW) Now I have a few questions to ask about your membership in each of these organizations.

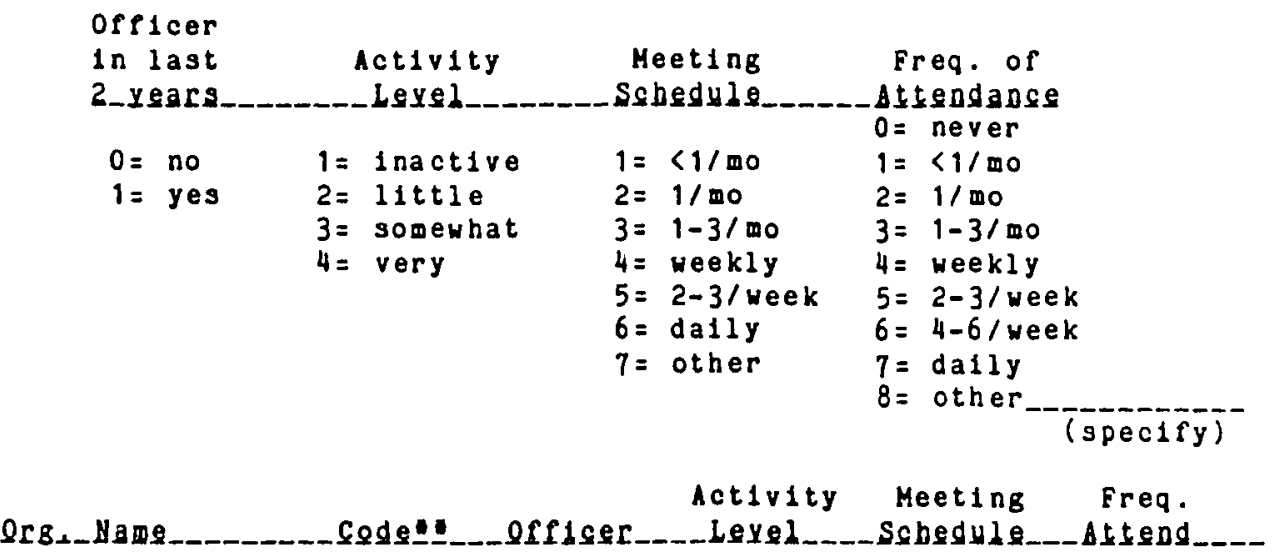

\footnotetext{
- "organization CODE

$0=$ none

1 = business, prof or tech

2 = church connected

$3=$ charitable

$4=$ civic, community or nelghborhood 10 other._._..._(specify)

5 = fraternal or veterans $99=01$

$6=$ political

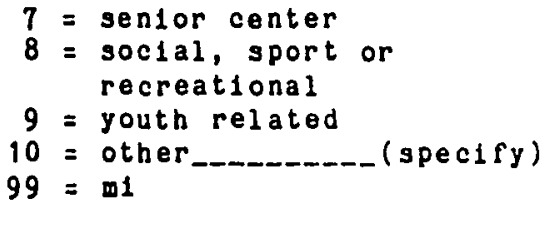

$7=$ sentor center social, sport or ecreat 1 ona 1
} 
29. Do you give your t1me to other volunteer work?

$0=$ no--1f no, skip to Q. 30

$i=$ yes

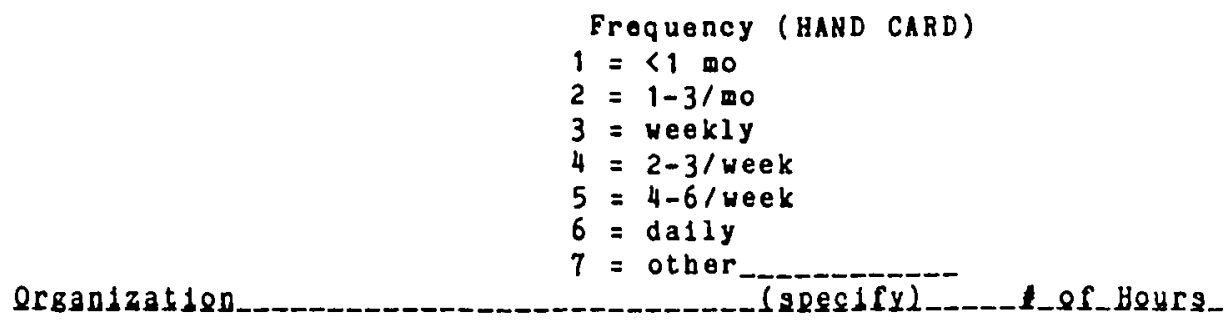

30. Do you have any special interests, pursuits, or hobbies? (PROBE: What do you do with your spare time?)

$0 \begin{array}{ll}\text { Frequency (HAND CARD) } \\ \text { no, nothing } & 1=\langle 1 \text { mo }\end{array}$

$2=1-3 / \mathrm{mo}$

$3=$ weekly

$4=2-3 /$ week

$5=4-6 /$ week

List

$6=$ dally

esponse

$7=$ other

Total hobbies

sum of hobbies freq. 

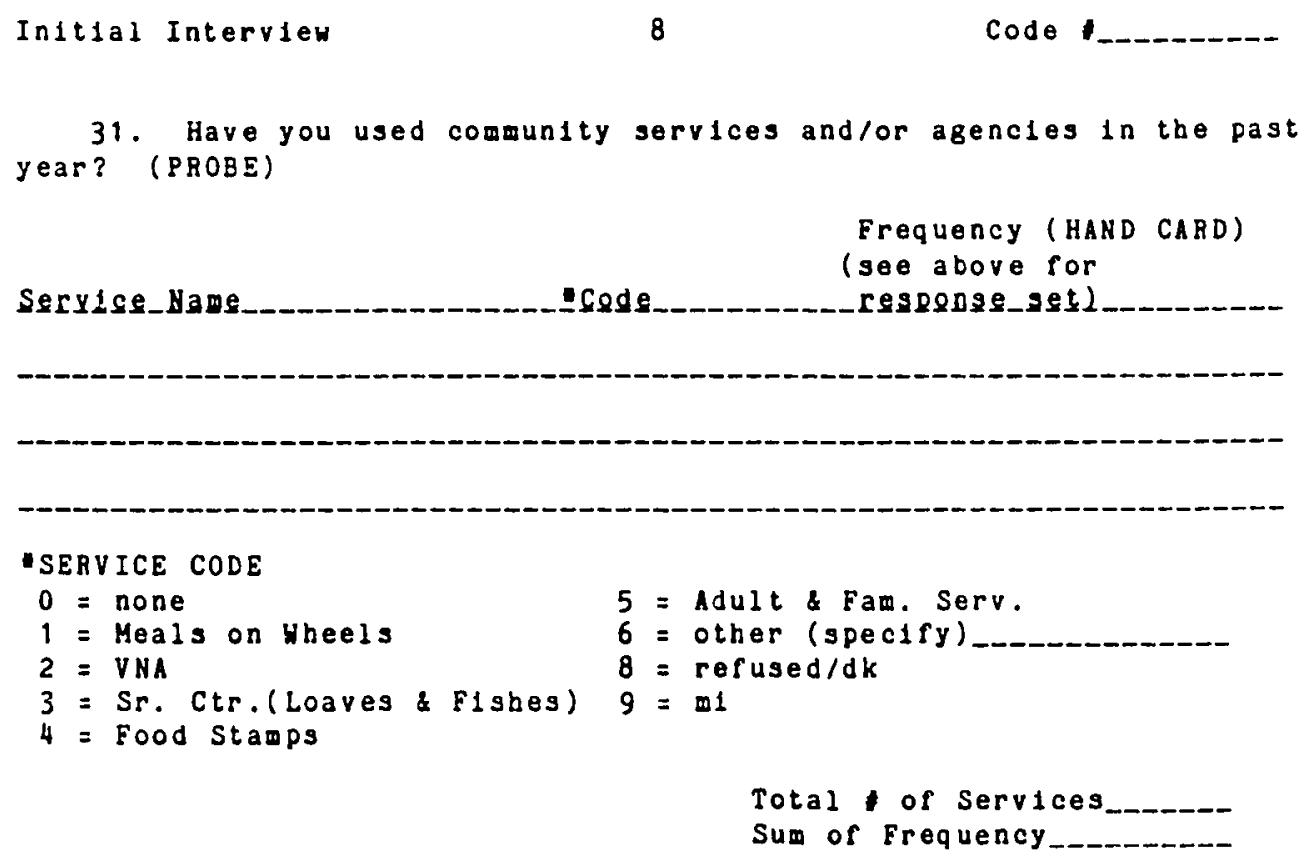

HEALTH/HELL-BEING :

In the following sections are questions regarding your health and well-being.

32. Here there any 1mportant good or bad events that happened to you in the year before the stroke? (FOR EXAMPLE: Death of someone close, birth of grandchild, retirement, serlous accident, etc.)

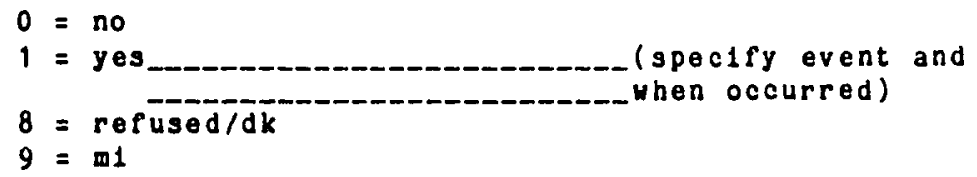

33. Did you have any physical disabilities that limited your work or social activities before the stroke?

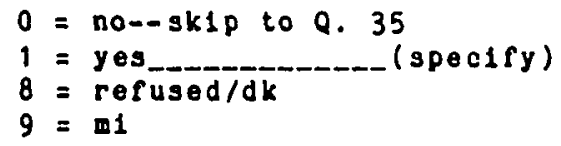

34. Does this _._._. require the use of special services, alds or equipment? (PROBE: For example, braces, vision or hearing a1ds, hand rails, hospltal beds, etc.)

$$
\begin{array}{ll}
0=\text { no--if no, probe } & 8=\text { refused } / d k \\
1=\text { yes_on } & 9=1 / n a
\end{array}
$$


35. How would you have rated your overall health before the stroke? (HAND CARD TO R)

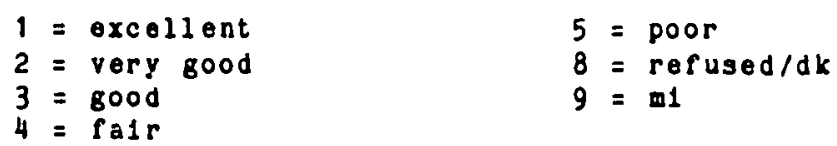

37. Do you feel that within the next six montbs your overall health is likely to (READ RESPONSES)

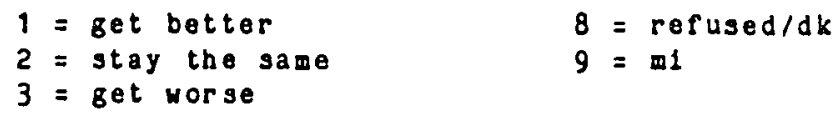

38. Do you bave any chronic bealth problems? (IF NO ANSWER, PROBE: e.g., Diabetes, beart condltion, high blood pressure)

$0=n_{0}$

$1=$ yes--11st:

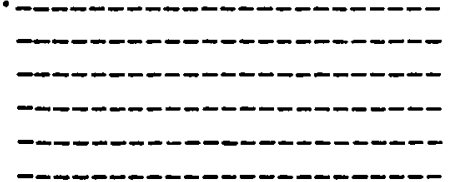

39. Other than for the stroke, bave you been hospitalized in the one year before the stroke?

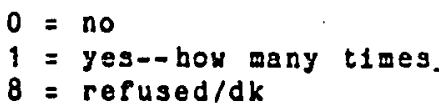

40. In the six months prior to the stroke, how many times have you visited the doctor?

Total _.._. (if 0 , ask Q. 41)

41. Prior to the stroke, when was the last time you saw a doctor?

42. Prior to the stroke, what medications were you taking on a regular basis?

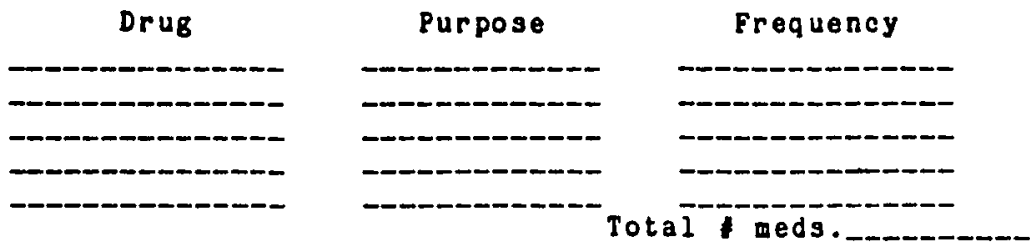


43. Are you taking any medicines now that you were not taking prior to the stroke?

\begin{tabular}{ccc} 
Drug & Purfoge & Frequency \\
\hdashline & & - \\
\hline
\end{tabular}

44. In the six months before the stroke, did you have an emotional problem for which you wanted help?

$$
\begin{aligned}
& 0=\text { no } \quad 8=\text { refused/dk } \\
& 1=\operatorname{yes}(\text { IF YES, ASK Q. 45) } 9=\text { mi }
\end{aligned}
$$

45. Did you recelve help for this concern?

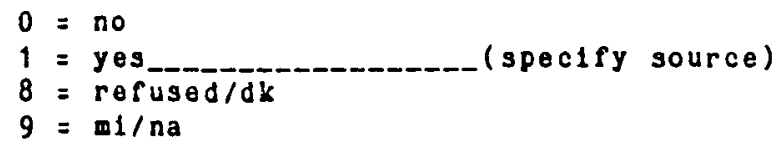

46. In the six months before the stroke, which of the following cholces best describes your appetite? (HAND CARD TO R)

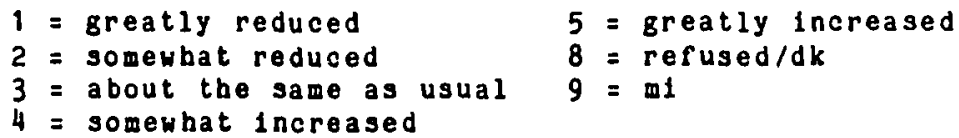

47. Did you experience any change in your sleeping habtts in the six months before the stroke?

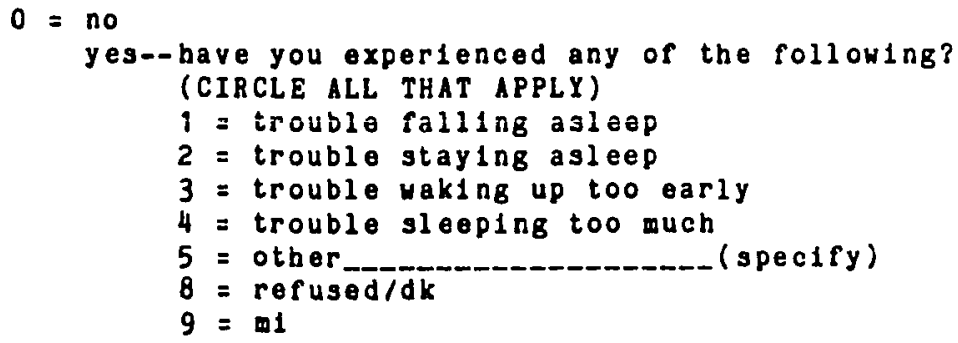

Now I'd like to ask you some questions about the stroke that you have recently experienced. 
48. Thinking back, can you think of anything you might have done to prevent the stroke?

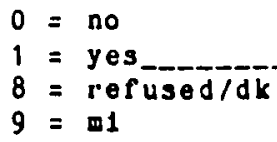

$1=$ yes

$8=r e f u s e d / d k$

$9= \pm 1$

49. How concerned are you about the possibility of another stroke? (HAND CARD TO R)

$$
\begin{array}{ll}
1=\text { very unconcerned } & 5=\text { very concerned } \\
2=\text { sowewhat unconcerned } & 8=\text { refused } / d x \\
3=\text { neutral } & 9=\text { m } 1 \\
4=\text { somewhat concerned } &
\end{array}
$$

50. In your view, how severe are your physlcal health problems nOW? (HAND CARD TO R)

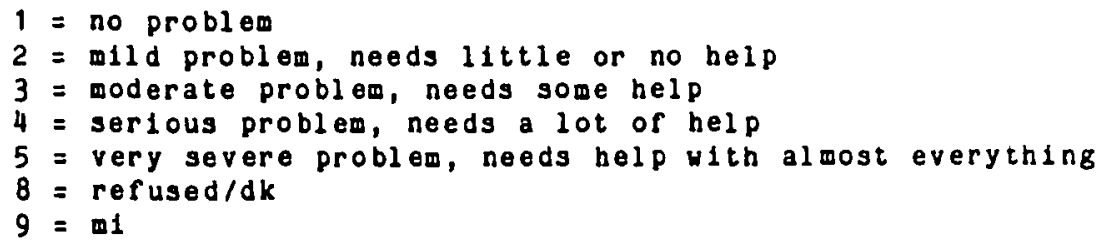

51. How concerned are you that care for you in the future? (HAND CARD TO $R$ )

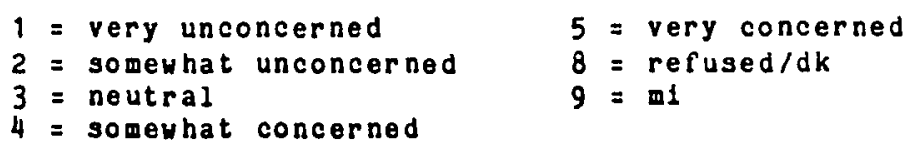

52. Who or what, so far, has been your greatest source of strength in helping you adjust to the stroke? (UP TO THREE SOURCES) (HAND CARD TO R)

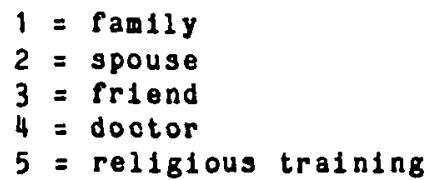


Intial Interview

Code

54. Have you known anyone else who has had a stroke?

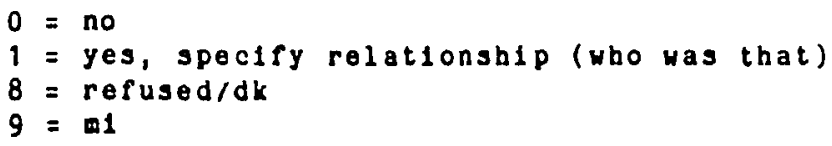

55. Who or what do you blame mest for the stroke happening?

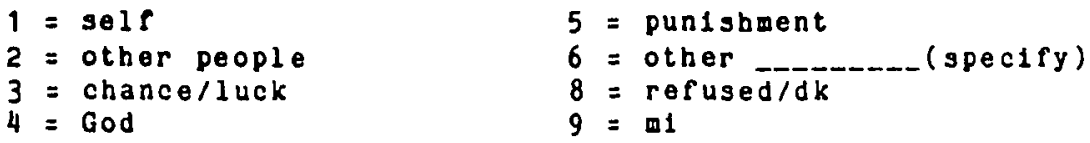

SPOUSAL/MARITAL RELATIONSHIP:

(Q. 56-67 TO BE ASRED ONLY IF SV/PI HAVE SPOUSAL/MARITAL RELATIONSHIP)

Now I want to ask a few questions about your relationship with --about some of the things you enjoy dolng together, and some of the ways you've spent tioe together. These next questions w111 be about your relationship prior to the stroke.

56. Before the stroke, how of ten did you and _........ engage in activities or outside interests together?

(HAND CARD TO R)

Have an Interesting conversation about ideas or opinions?

58. Laugh together?

59. Calmly discuss something? $0=$ never

$1=<1$ mo

$2=1-3 /$ mo

$3=$ weekly

$4=2-4 /$ week

$5=d a 1 l y$

$6=>d a t l y$

$8=$ refused/dk

$9=m 1 / n a$

60. Work together on a project? 
61. Before the stroke, did you and belong to any of tho same organizations? (SPECIFY NAME AND CODE BY TYPE OE ORG AN IZATION ) :

$\begin{array}{ccc}\text { Name of } & \text { EType of } & \text { Freq. of Jolnt } \\ \text { Organization } & \text { Organization } & \text { Attendancelper wk. basts }\end{array}$

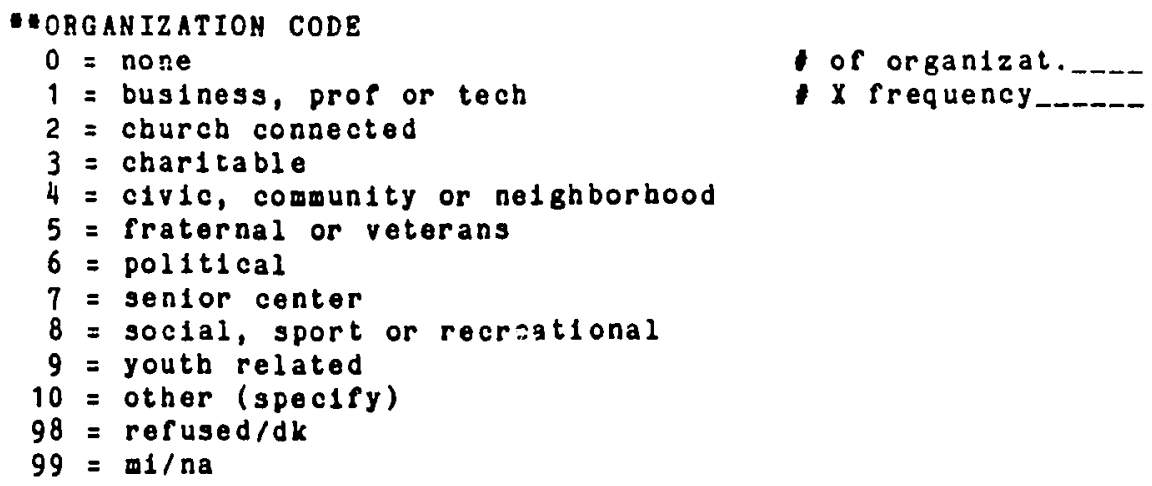

62. In describing your relationsh1p with _..... over the years, please tell we how often the two of you would be $11 \mathrm{kely}$ to disagree on the following:
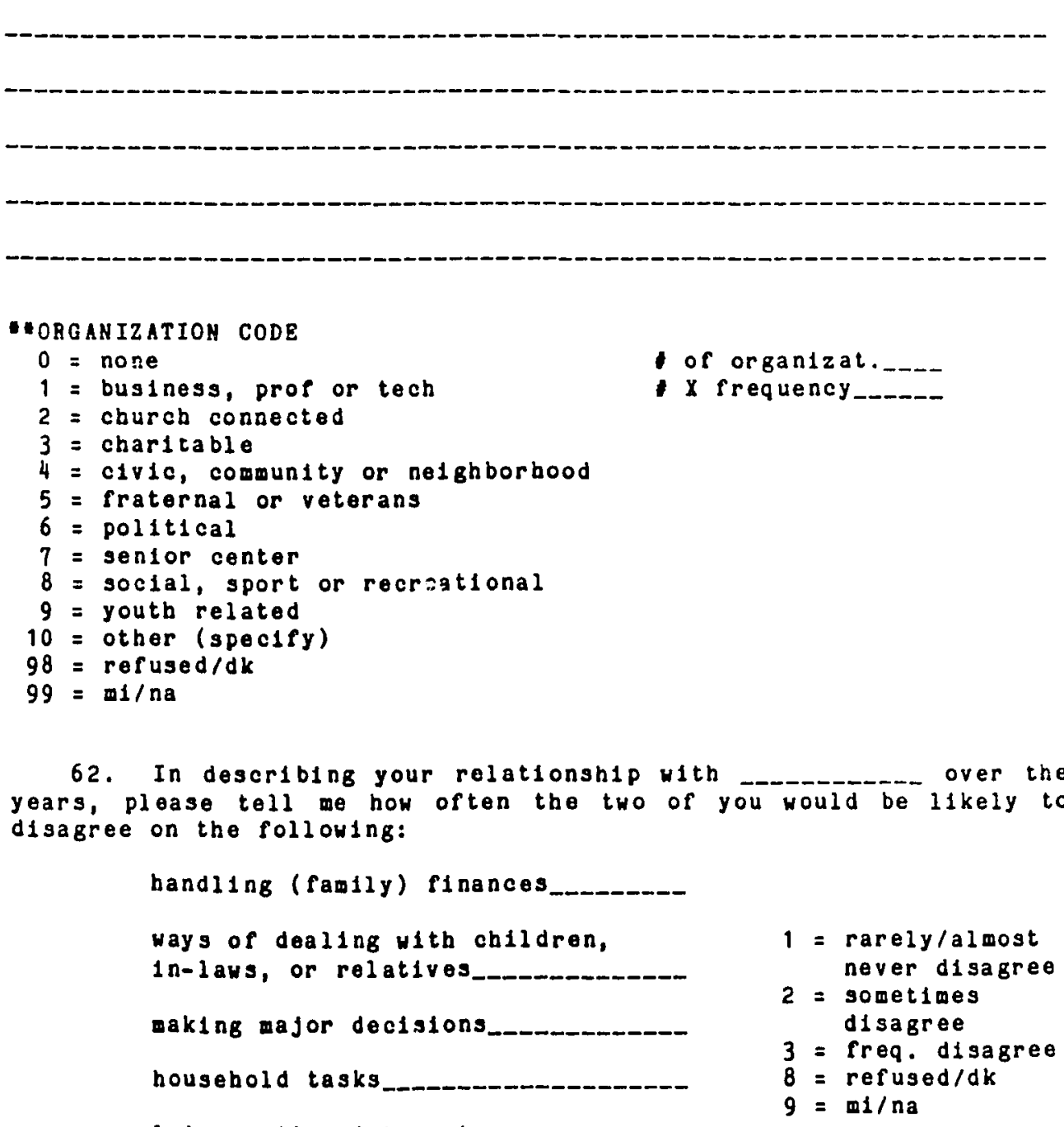
63. In your family, who has beld tibe primary respongibliliy for:

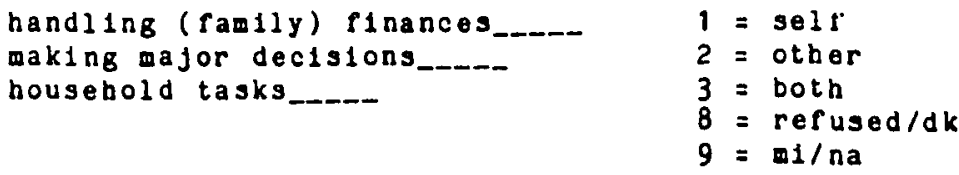

64. Think about your relationghip with

Before the stroke, if you had a very personal and serlous problem, how likely would you be to discuss it with (HAND CARD TO R)

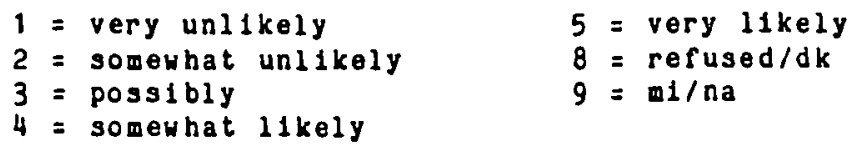

65. Agaln, before the stroke, if _...... had a very personal and serlous problem, how likely would he/she be to discuss it with you? Would you say it was (HAND CARD TO R)

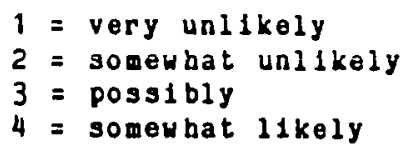

$5=$ very $11 \mathrm{kely}$

$8=\mathrm{refused} / \mathrm{dk}$

$9=m 1 / n a$

66. In general, how happy are you with your marriage (relationship)? (HAND CARD TO R)

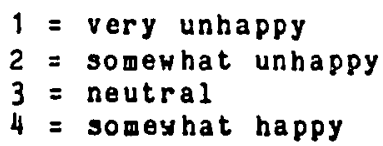

67. Compared to other couples you have known, overall, would you rate your marriage (relationship) as worse, about the same as, or better than other couples?

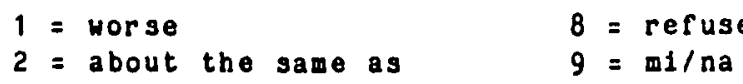

SOCIAL NETWORR-SOCIAL SUPPORT DATA: BEFORE THE STRORE:

Now I'd like to ask some questions about family, friends, and social activities before the stroke. First, I want to get as accurate a picture as possible about the people you felt closest to, spent time with, depended upon for help, or who depended on yoy for belp before the stroke. 
68. In the past six montbs, about how often have you:

(HAND CARD) Frequency Code

$$
\begin{aligned}
& 0=\text { never } \\
& 1=\langle 1 \text { mo } \\
& 2=1-3 / \text { mo } \\
& 3=\text { weekly } \\
& 4=2-3 / \text { week } \\
& 5=\text { dally }
\end{aligned}
$$

$15 \pm 1 \times 1 \pm 1$

(1) Had someone to your home for lunch or dinner

(2) Gone to someone's howe for lunch or dinner

(3) Had someone come by your house to visit

(4) Gone over to someone's house for a visit

(5) Gone out with someone (to a restaurant,

Gone out with someone (to a restaurant, movie, etc.)

69. Sometimes friends, nelghbors, or famlly members perfora belpful tasks for each other, such as watching the house when one is out of town, plcking up the mall, helping with minor household repalrs or chores, etc. Are there people who would help you in these ways?

$$
\begin{aligned}
& 0=\text { no } \\
& 1=\text { yes }
\end{aligned}
$$

(IF YES, ASK FOR FIRST NAMES AND RECORD ON NAME LIST I. FOLLOW SAME FORMAT AND RECORD NAMES FOR Q.'S 70-83.) ways?

70. Are there people who would ask yoy to help them in these

71. Thinking back to the six montbs tefore the stroke, do you have any friends, nelghbors, or ramily members who would help you if you were sick for a short time? That $1 \mathrm{~s}$, for a few days up to a week?

72. How about people who would ask you to help them if they were sick for a short t1me? Do you have any friends, nelghbors, or family members who would ask for your help in this way?

73. When you are concerned about a personal matter, for example, about someone you are close to, or something you are worried about, bow often do you talk about it with someone? Would you say, 
74. Ttist aboit tho last 31z moaths, aspacialij tine tide berore the stroke, when you did talk with someone about personal matters, whow did you talk with?

75. Are there people who would come to you to talk about something they were worried atout, or about a personal matter? iemember, it can be a family member, friend, or nelghbor.

76. Often, people ask advice, or rely on the judgment of someone they know in making important decisions about thelr lives--for example, about thelr family or their work. Is there anyone whose opinion, in the six months before the stroke, you considered seriously in making important decisions?

77. Are there people who would seek your oplnion or advice about things?

78. Sometimes people get together with close friends, neighbors, fawlly or others to do something enjoyable together, or just to soclalize. May I have the first names of the persong you've done (or do) these things with on a regular basis?

79. Sometimes peoplo get together with others to talk about hobbles or spare-time interests they have in common. Do you ever do this? With whom?

80. If, In the t1me before your stroke, you had been 1ll, or needed help for longer than just a few days, say weeks or months, $1 \mathrm{~s}$ there someone, or more than one person, you could call on to help, come and stay with you if needed, or take you into their home?

81. Sometimes, people have one person, or a few people they feel closest to-mpople they can confide in, or share personal worries and concerns with. Who are the people you feel closest to in this way?

82. (IF SPOUSE IS HOT NAMED, ASK ABOUT SPOUSE) WOUId you say that you feel closer to this person (these people) than your spouse?

$$
\begin{aligned}
& 0=\text { no } \\
& 1=\text { yes }
\end{aligned}
$$


Code

83. (PRESENT NAME LIST I)

Are there any other people who were important to you before the stroke whose names are not on thls list? For example, friends, relatives, netghbors, co-workers, or members of the same organization?

(ADD ANY ADDITIONAL NAMES TO NAME LIST I)

SOCIAL NETWORK DATA: STRORE VICTIH'S SUPPOHT-TIME OF STROKE:

Now I'd like to ask about those friends, co-workers, nelghbors or famlly members who have been helpful to you since the stroke. This can be practical help, moral support, encouragement, etc.

100. Who are the people who've been important and helpful to you during this time of the stroke? (RECORD ON NAME LIST A)

\section{SOCIAL NETHORK DATA - NEgative aspects:}

In situations like th1s, sometimes we find that there are people who don't help as wuch as we expect them to, or who don't help at all when we expected them to.

101. Are there people who have helped during this time of 11 ness, but not as wuch as you expected they would, or who haven't helped at all when you thought they would?

May I have their f1rst names? (RECORD NAMES AND OTHER INFORMATION BELOW )

Relationsip Helped less
tonsy


102. We all know people who try to belp, but end up aaking tbe situation worse. Are there any peoplo you feel bave been a bindrance to your recovery thus far even though they try to be belpful?

$$
\begin{aligned}
& 0=\text { no } \\
& 1=\text { yes }
\end{aligned}
$$

What are the1r names? (RECORD MAMES AND OTHER INFO BELOW)

Relationsh1p

Nage____

103. Sometimes we run 1 into peoplo who seen to be out to make life difflcult. Are there people like that who have made problems for you?

$0=$ no
$1=$ yes

Who would they be? (RECORD NAMES, RELATIONSHIPS BELOW)

yame

Belationsb10_to_Sy 
Initial Intorview
Code

104. We all know peoplo we find 1 teasy to get along with, and others we don't get along with. Are there people you don't get along with?

$$
\begin{aligned}
& 0=\text { no } \\
& 1=\text { yes }
\end{aligned}
$$

May I bave their first names? (RECORD HAMES AND RELATIONSHIPS BELOW)

INSTRUCTIONS FOR COMPLETING YAME LISTS, CODING SHEETS, AND MATRIX:

1. FOR NAME LIST I, RECORD INFORMATION REGARDING RELATIONSHIP, AGE, SEX, AHD CLOSENESS ON NAME LIST I FORM.

2. REPEAT FOR NAHE LISTS II AND A.

3. ON CODING SHEET, RECORD NAMES OF UP TO 10 PERSONS IN APPROPRIATE BOXES ICROSS THE TOP, BASED UPON THE FOLLOWING CRITERIA :

a. THOSE WHOM RESPONDENT INDICATES THEY (OR SV) ARE ESPECIALLY CLOSE TO.

b. THOSE RESPONDENT INDICATES AS MOST IMPORTANT PROVIDERS OF SUPPORT, IF NOT ALREADY SELECTED.

4. IF 10 OR FEHER HW MEMBERS, ASK QUESTIONS ON CODING SHEET ABOUT ALL OF THOSE NAMED.

5. COMPLETE IHPORMATION ON CODING SHEETS, USING APPROPRIATE SHEETS FOR PI AND SV.

6. CIRCLE MAMES THAT IPPEAR ON BOTH SV AND PI CODING SHEETS.

7. COMPLETE INFORHATION ON HH MATRIX SHEET. 
105. In general, how satisfled are you with the gmeunt or social contact you bave with otbers? (HAND CARD TO R)

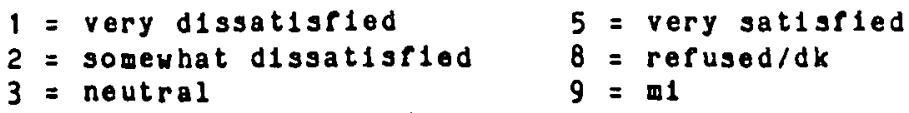

106. In general, how satisfied are you witb the quality or closeness of the social contact you have with others? (HAND CARD TO R)

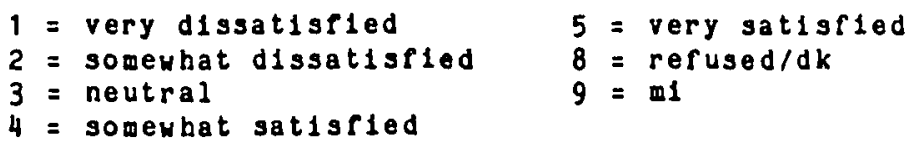

107. As you can seo, I've asked you many different kinds of questions about you. But everyone 13 different and I might have left out something that is important to you. Can you think of anytbing that I should bave asked about you and the stroke but didn't? 
Current Residence (SV only)

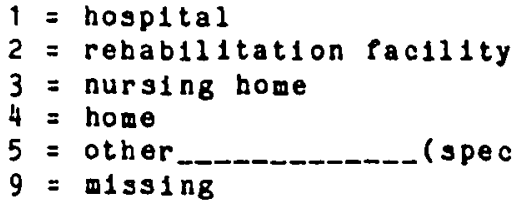

Was anyone else present during tbis interview?

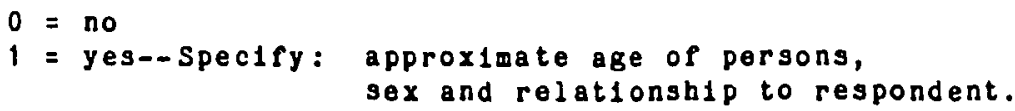

How long was this person present?

Did this person participate in interviow responses?

How well do you feel the respondent understood the litens of this interview?

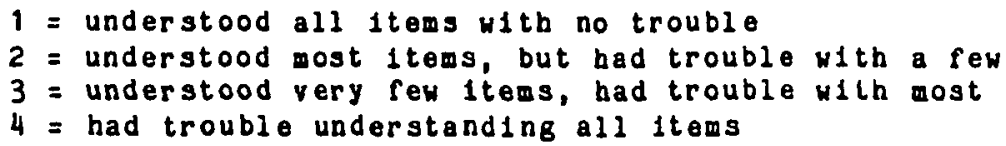

Note below any otber unusual problems in th1s interview: 
STROKE SURVEY: SOCIAL SUPPORT AND WELL-BEIHG

INITIAL INTERVIEW SCHEDULE:

PRIMARY INFORMANT FOR STROKE VICTIM

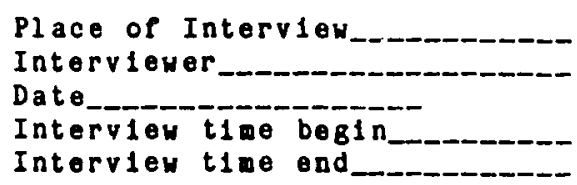

The first set of questions will cover general background

DEMOGRAPHICS:

3. What is his/her date of birth?

4. SEX (INTERVIEWER CODE)

$1=\operatorname{tenale}$

$2=\operatorname{mal} \theta$

5. What is his/her ethnic background? (ASK ONLY IF NOT OBV IOUS )

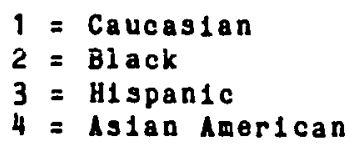

\author{
.
}


8. Does have any children, stepchildren, or adopted ch1ldren? $0=$ no Total of children:

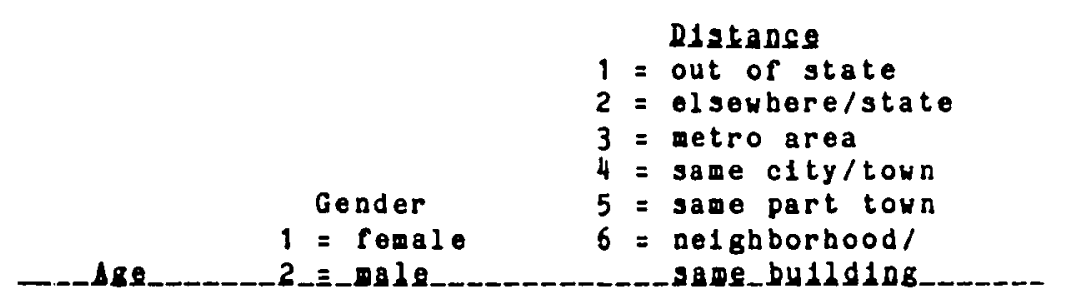

chlld_1

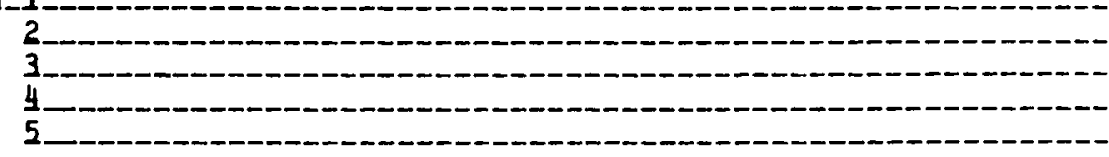

(USE OTHER SIDE IF > 5 CHILDREN)

9. Makeup of Household:

I'd like to ask sowe questions about the makeup of -_-_- 's housebold, about the people who live with bio/ber. What are the first names of all the otber persons who live with regular basis? (LIST BELOH) Name Sex (If_Det_obyloys)

Age *relat. to SV ERelat. to PI

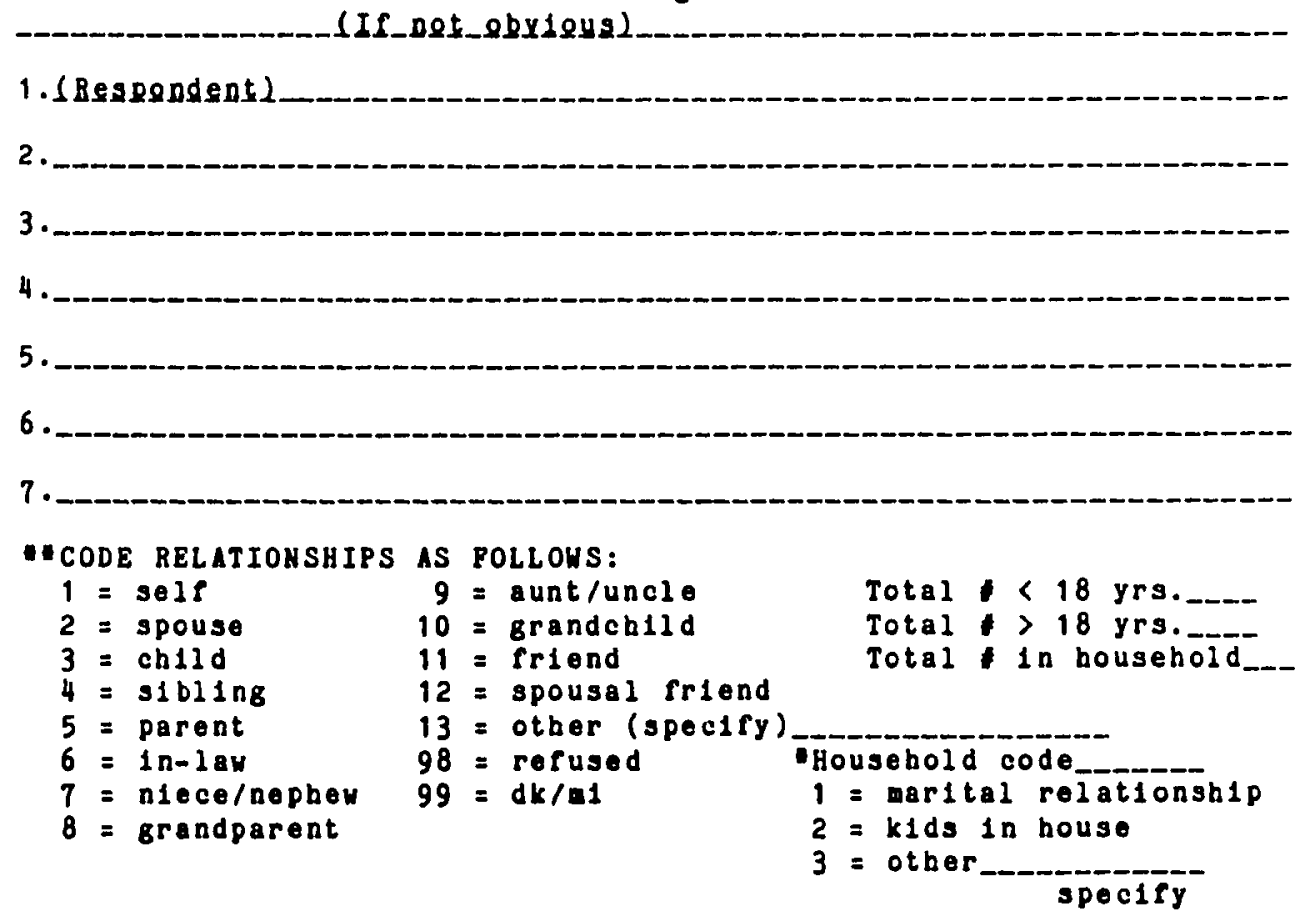


10. What 1s the highest level of education _._._. has completed?

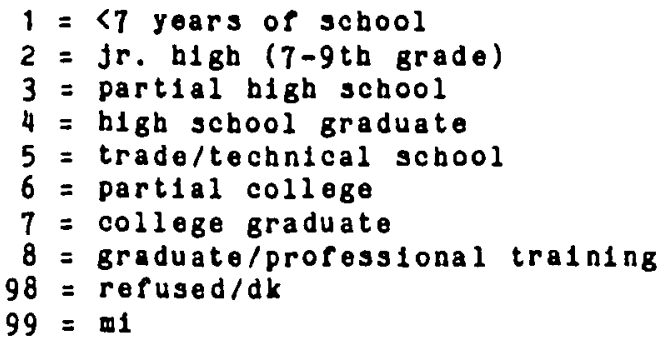

Now, I'd like to ask you some questions about _._._._. 's employment history.

11. At the t1we of the stroke, what was his/ber employment status? (HAND CARD TO R)

$0=$ never worked outside home--sk1p to $Q .15$.

1 = employed rull-time

$2=e m p l o y e d$ part-time

3 = unemployed

$4=$ retired

$5=$ other

$8=$ refused/dk

$9=m 1$

12. How Iong has been 8 years (months)

13. What 1s (was) _______'s occupation called? (PROBE: self employed/own business)

15. In which of the following categories did year's total beysebeld income fall? (HAND CARD TO R)

$$
\begin{aligned}
& 1=\$ 5,000 \\
& 2=\$ 5,000-\$ 9,999 \\
& 3=\$ 10,000-\$ 14,999 \\
& 4=\$ 15,000-\$ 19,999 \\
& 5=\$ 20,000-\$ 24,999 \\
& 6=\$ 25,000-\$ 29,999
\end{aligned}
$$

$$
\begin{aligned}
7 & =\$ 30,000-\$ 39,999 \\
8 & =\$ 40,000-\$ 49,999 \\
9 & =>\$ 50,000 \\
98 & =\text { refused/dk } \\
99 & =\text { m1 }
\end{aligned}
$$

The next set of questions w1ll be asking you about I1ving arrangements prior to the stroke. (ASK 16-20 OF SV ONLY IF NOT ALREADY DETERMIHED.)

16. How long has lived at bis/her current residence? 
Code

17. Is (was) bis/ber residence in an urban, rural or suburban sotting? (INTERVIEWER CODE)

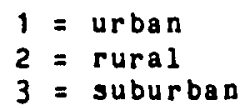

18. In wat type of residence do (d1d) _tive? (INTERVIEWER CODE)

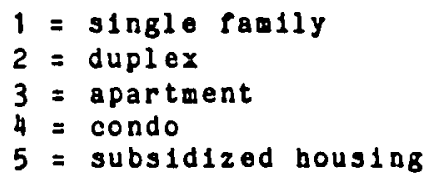

19. Do (d1d) own or rent the residence?
$1=$ own
$8=$ rerused $/ d k$
$2=$ rent
$9=m 1$

20. How Iong has Iived in the area?

-

21. What forms of transportation did____ utilize; bow did he/she run errands, visit friends and relatives, etc.? (CIRCLE ALL THAT APPLY)

$$
\begin{aligned}
& i=\text { public } \\
& 2=\text { sriends } \\
& 3=\text { relatives } \\
& 4=\operatorname{tax} 1
\end{aligned}
$$

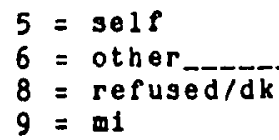

22. Does have an ALL THAT APPLY)

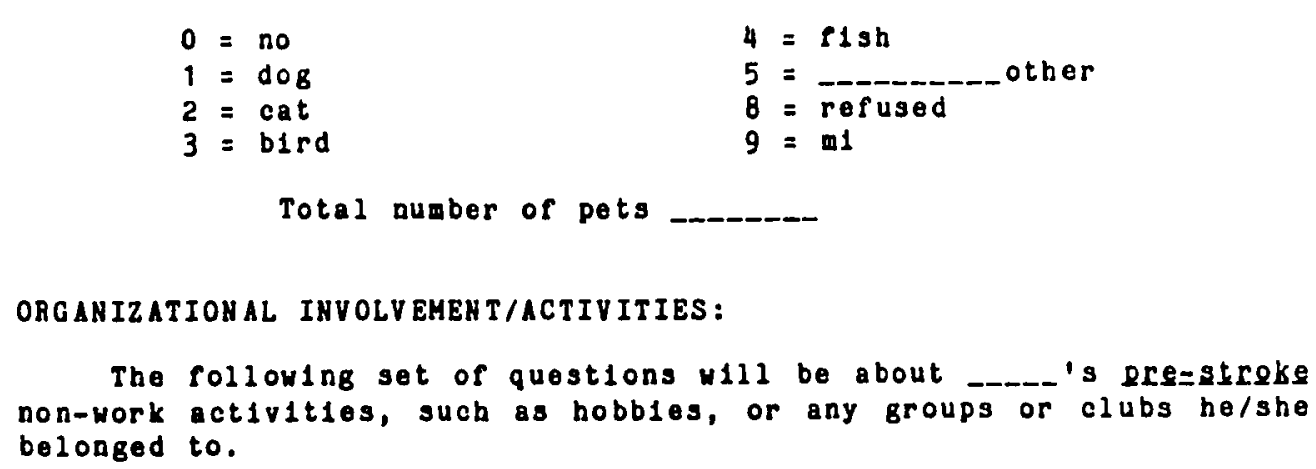

The following set of questions w1ll be about _-_-'s pre=stroke non-work activities, such as hobbles, or any groups or clubs he/she belonged to. 
25. What is 's rel1g1ous proference?

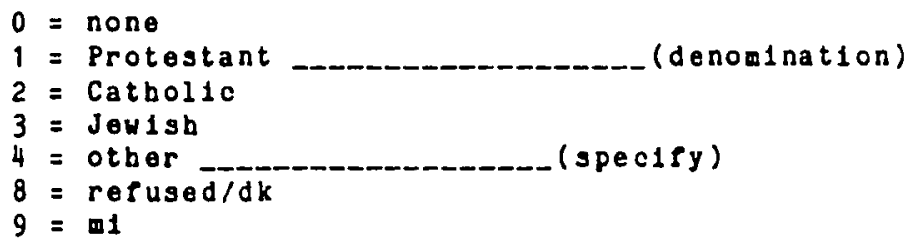

26. Before the stroke, how active was _._._. In bis/her religlous organization? (READ RESPONSES)

1 = Inactive

2 = only a little active

3 = somewbat active

$$
\begin{aligned}
& 4=\text { very act } 1 v e \\
& 8=\text { refused } / d k \\
& 9=m 1
\end{aligned}
$$

27. How often did attend religlous services?

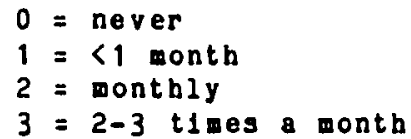


Inttal Interview
6
Code

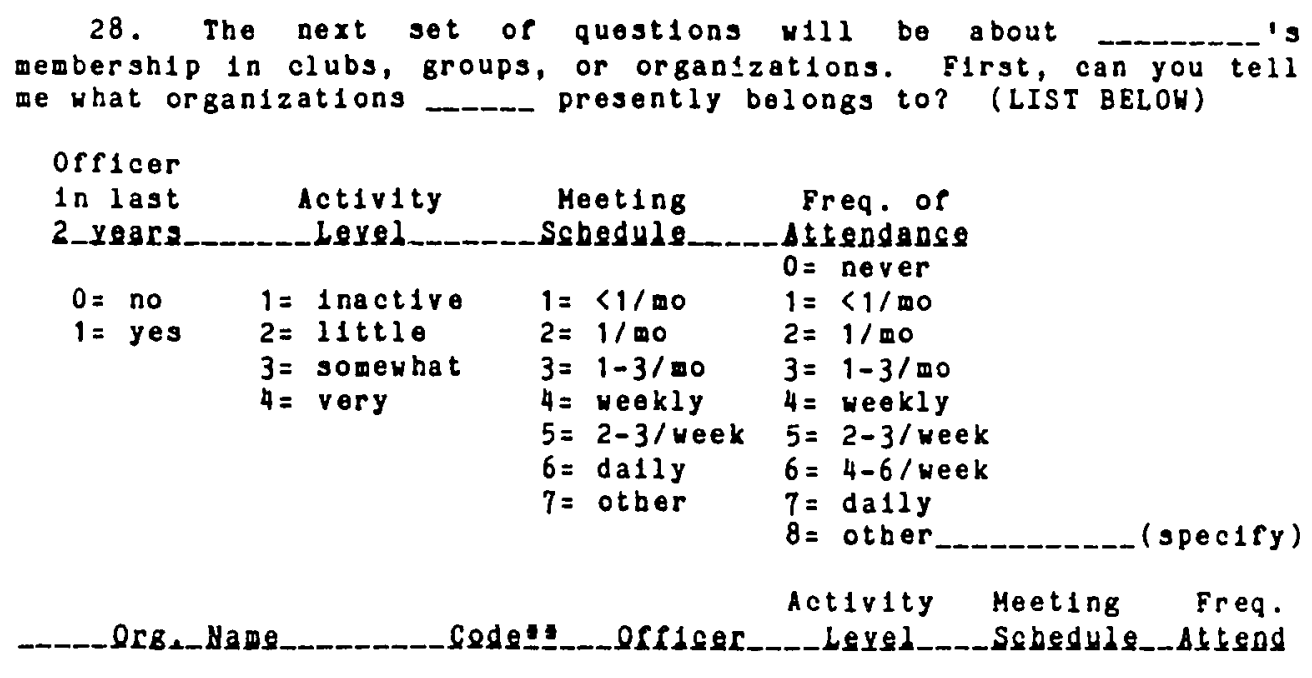

28. The next set of questions w1ll be about _-_-_'s membersh1p in clubs, groups, or organizations. First, can you tell me what organizations presently belongs to? (LIST BELOH)

officer in last

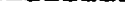

$0=$ no
$1=1$ nact 1 re

$2=11 \mathrm{ttl} \theta$

$2=1 / \mathrm{mo}^{\circ}$

$3=1-3 /=0$

$=$ weekly

$7=$ otber Leyel

$0=$ never

$=\left\langle 1 / \mathrm{mo}^{\circ}\right.$

$2=1 / \mathrm{mo}$

$3=1-3 / \mathrm{m}$

$=$ weekly

$7=d a 11 y$

$8=$ otber

Meeting Freq.

OCB__dame Code:__oft1eer . 
Intital Interview

29. Does g1ve h1s/ber tiae to other volunteer work?

$0=$ no--if no, skip to $Q .30$

$1=$ yes

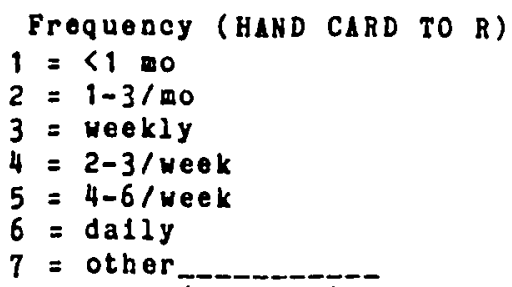

ersandzation__.

30. Does _._._ have any special laterests, pursuits, or hobbles? (PROBE: What does he/she do with their spare time?)

$\begin{array}{ll}0=\text { no, nothing } & \text { Erequency (HAND CARD TO R) } \\ 1 & =31 \text { mo } \\ 2 & =1-3 / \text { mo } \\ 3 & =\text { weekly } \\ 4 & =2-3 / \text { week } \\ 5 & =4-6 / \text { week } \\ 6 & =\text { datly } \\ 7 & =0 \text { ther }\end{array}$


31. Has the past year? (PROBE)

used communty sorvices and/or agencies in

Erequency (HAND CARD)
(see above for
Serydeg_Name_esgenge_set)

- SERVICE CODE

$0=$ none, no

1 = Meals on Wheels

$2=$ VNA

$3=\operatorname{Sr}$. Ctr. (Loaves \& Fishas)

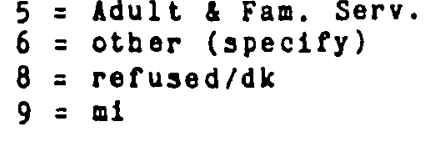

Total or Services

sum of frequency

HEALTH/WELL-BEING :

In tbe following sections are questions regarding is health and well-belng.

32. Here thore any Important good or bad ovents that happened to someone close, birth of gradichlld, retirement, serlous accident, etc.)

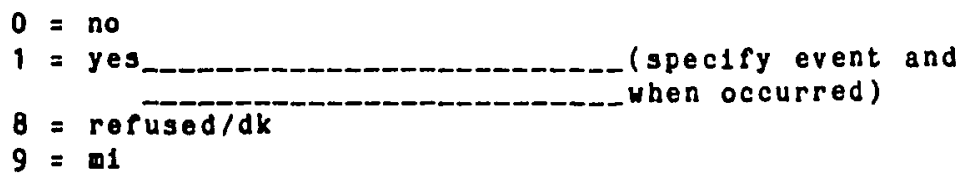

33. D1d _._. bave any physical disabilities that limited work or social activities before the stroke?

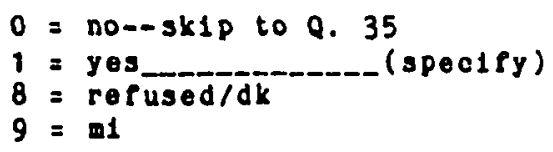

34. Did this _._._. require the use of special services, alds or equipment? (PROBE: For example, braces, vision or hearing alds, hand ralls, hospital beds, etc.)
0 a no-nif no, probe
$1=$ yes (spocify)
$8=\operatorname{refused} / d k$
$9=a 1 / n a$ 
Code

35. How would you rate 's overall health prior to the stroke? (HAND CARD IN R)

$\begin{array}{ll}1=\text { excellont } & 5=\text { poor } \\ 2=\text { rory good } & 8=\text { refused } / d k \\ 3=\text { good } & 9=01\end{array}$

38. Did _. have any chronic bealth problems previous to this siroke? (IF NO ANSWER, PROBE: e.8., Diabetea, beart condition, high blood pressure)
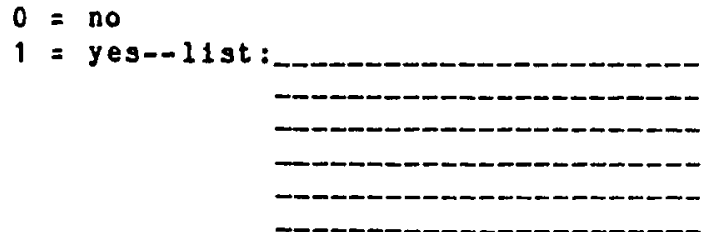

39. Otber than for tho stroke, bas been hospitalized in tho year before the stroke?

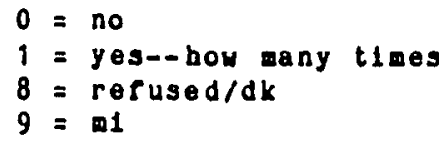

length of hospitalization (days) for each

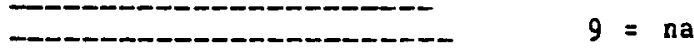

40. In the six months prior to the stroke, how wany times has visited the doctor?

Total (18 0, ask Q. 41)

41. Prior to the stroke, when was the last time saw a doctor for bis/her oun bealth?

42. Prior to the stroke, what medications was taking on a regular basis?

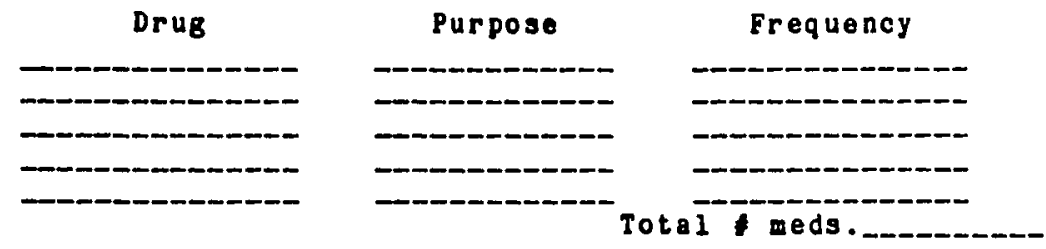


45. In the six montbs before the stroke, did bave an emotional problem for whicb he/she sought bolp?

$$
\begin{array}{ll}
0=\text { no } & 8=\operatorname{rerused} / d k \\
1=\text { yes (specify source) } & 9=\operatorname{ml} / \mathrm{na}
\end{array}
$$

46. In the six montbs before the stroke, which of the following cholces best describes 's appetite? (HAND CARD TO R)

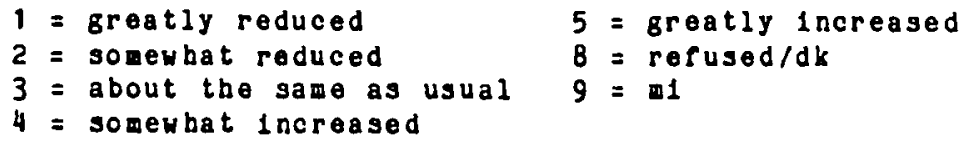

47. D1d experience any change in sleeping habits in the six wonths before the stroke?

$0=$ no yes-- bave you experlenced any of the following? (CIRCLE ALL THAT APPLY)

1 = trouble falling asleep

2 = trouble staylng asleop

3 = trouble waking up too early

4 = trouble sleeping too much

$5=$ other (specify)

$8=$ refused/dk

$9=m 1$

STROKE--PERCEPTIONS \& FEELINGS:

Now I'd like to ask some questions about the stroke.

48. Thinking back, can you think of anything _._. olght bave done to prevent the stroke?

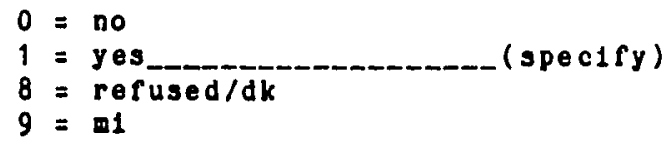

49. How concerned do you think _._. 1 s about the possibility of another stroke? (HAND CARD TO R)
1 = very unconcerned
$5=$ very concerned
2 = somewhat unconcerned
3 = neutral
$8=\mathrm{refused} / \mathrm{dk}$
$4=$ sonewbat concerned
$9=m 1$ 
54. Has known anyone else who bas had a stroke?

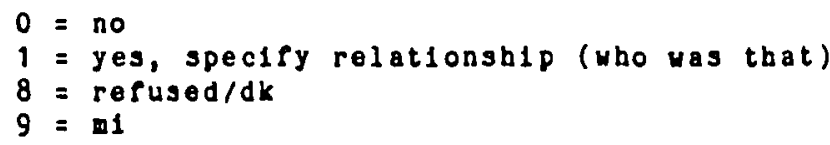

SOCIAL NETWORK--SV BEFORE STRORE:

How, Lew questions about the people, relationships, and activities that were important to _._._._- before the stroke.

68. In the past s1x months, about how often did

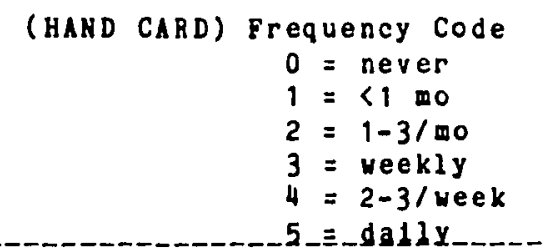

(1) Have someone to bls/ber bowe for luncb or dinner

(2) Go to someone's home for lunch or dinner

(3) Have someone come by h1s/her house to visit

(4) Go over to someone's house for a v1sit.

(5) Go out with soneone (to a restaurant, movie, etc.)

84. Lre there people - regularly spent time socializing with, visiting, sharing hobbles or lnterests?

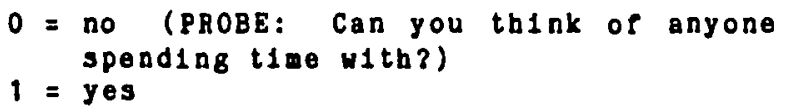

May I have the first nawes of these people? (ADD NAMES TO HAME LIST 1)

(FOLLON SAME FORHAT AND RECORD NAMES FOR 0.'S 85-86)

85. If be/she talk to? had serlous concern or problem, whom would

86. (PRESENT MAHE LIST A)

Are there any other people who were important to before the stroke whose names are not on this list? For example, frlends, relatives, nelgbbors, oo-workers, or menbers of the same organizat1 on? 
Code

87. Lre tbere poople

didn't get along with? Who would they be?

Name

Belat1enghid_to_sy

SOCIAL NETWORK DATA, VICTIM--POST STROKE:

100. Who are the people who have been important and helpful to during this time of experiencing a stroke?

(ADD ANY ADDITIONAL NAMES TO MAME LIST A)

INSTRUCTIONS FOR COHPLETING WAHE LISTS, CODING SHEETS, AND MATRIX:

1. FOR NAME LIST I, RECORD INFORMATION REGARDING RELATIONSHIP, AGE, SEX, AND CLOSENESS ON HAME LIST I FORM.

2. REPEAT FOR NAME LISTS II AHD $A$.

3. ON CODING SHEET, RECORD HAMES OF UP TO 10 PERSONS IN APPROPRIATE BOXES ACROSS THE TOP, BASED UPON THE FOLLOHING CRITERIA :

a. THOSE WHOM RESPONDENT INDICATES THEY (OR SV) ARE ESPECIALLY CLOSE TO.

b. THOSE RESPOHDEYT IHDICATES AS MOST IMPORTANT PROYIDERS OF SUPPORT, IF NOT ALREADY SELECTED.

4. IF 10 OR FEUER NW MEMBERS, ASK QUESTIONS ON CODING SHEET ABOUT ILL OE THOSE NAMED.

5. COMPLETE IHPORMATION ON CODING SHEETS, USING APPROPRIATE SHEETS FOR PI AHD SV.

6. CIRCLE naMES that LPPBAR ON BOTH SV AND PI CODING SHEETS.

7. COMPLETB IHFORHATION ON NH MATRIX SHEET. 
1.1). No

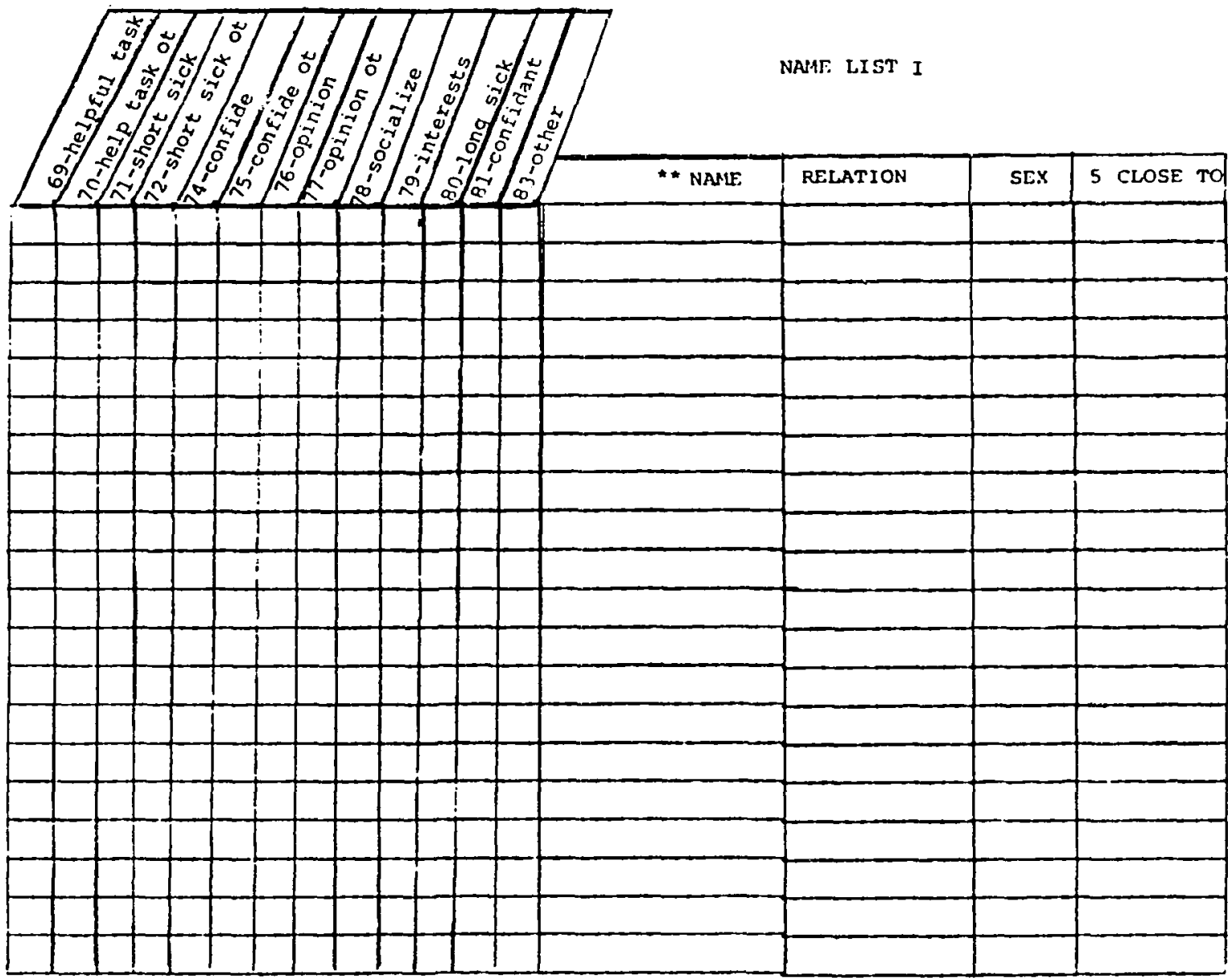

Relationship Code 0 -other

1-spouse

2-child

3-sibling

4 -parent

5-other relative

6-neighbor

7-work associate

8 -friend

Sex code

1 -female

2 -male

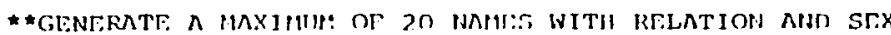
INFORMATION UN FAC?I 
I.D. No.

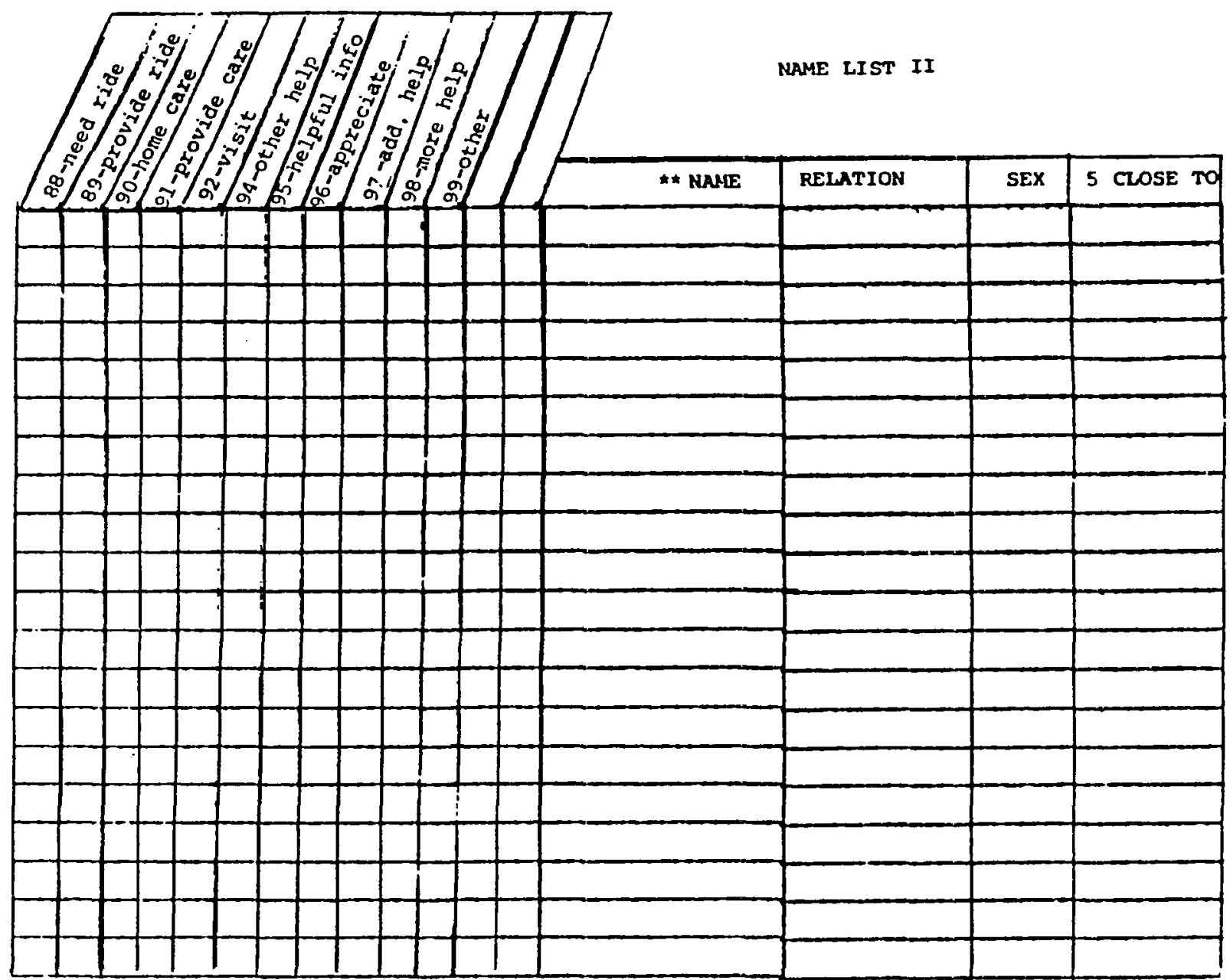

Relationship code

o-other

1 -spouse

2-child

3-sibling

4-parent

5-other relative

6-neighbor

7 -work associate

8-friend

Sex code

1-female

2-male

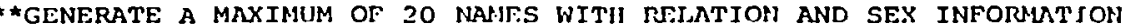
ON EACH 
I.D. No

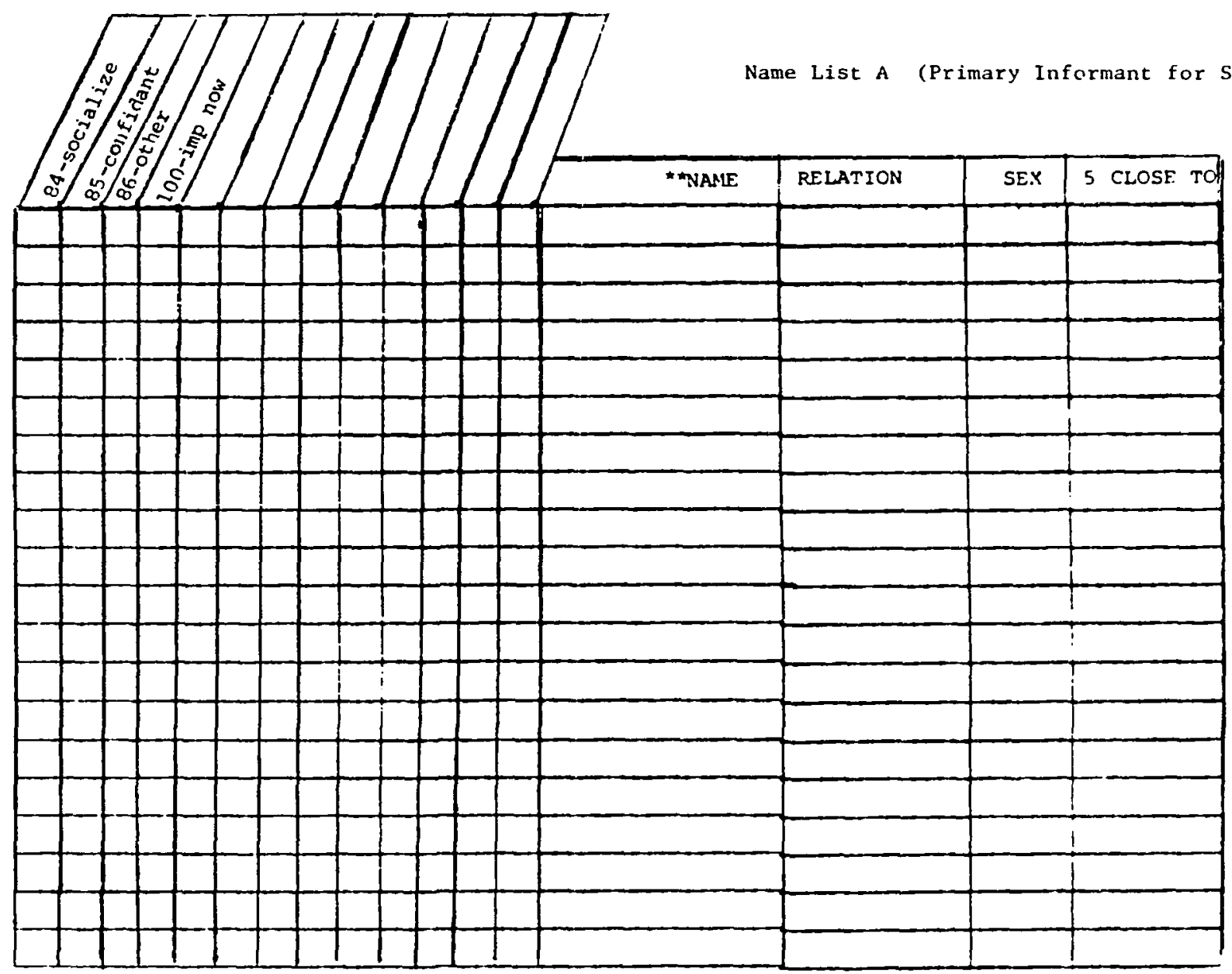

Relationship Code 0 orther

1 -spouse

$2-$ child

3-sibling

4-parent

5 -other relative

6-neighbor

7-work associdte

8-friend

Sex code

1-female

2-male

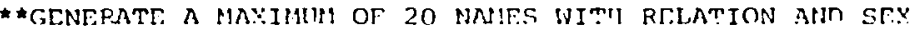
INFORIATION ORI EACH 
(Circle all names that appear on both SV and PI coding sheets)

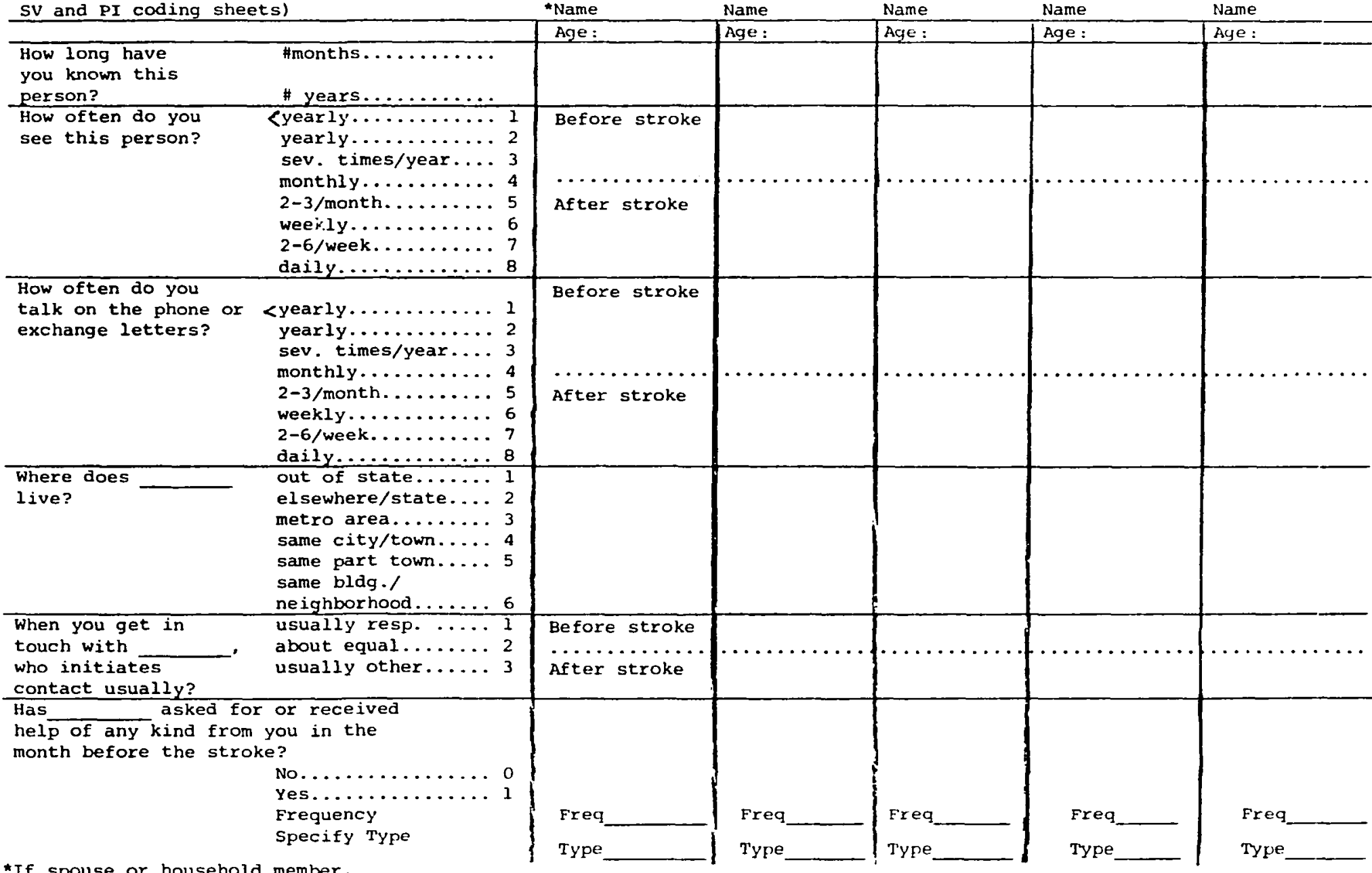

"If spouse or household member,

delete column 


\section{NETHORK MATRIX FORM}

LIST THE 10 SELECIED NAMES DOHN THE COLUMM;LIST THE FIRST 9 OF THEM ACROSS IHE TOP, III THE SAME ORDER. III THE SPACES PROVIDED, ASK ABOUT ALL RELATIOIISIIIPS IN COLUMI 1, TIEN ABOUT ALL RELATIONSIIPS IN COLUMH 2, ETC.

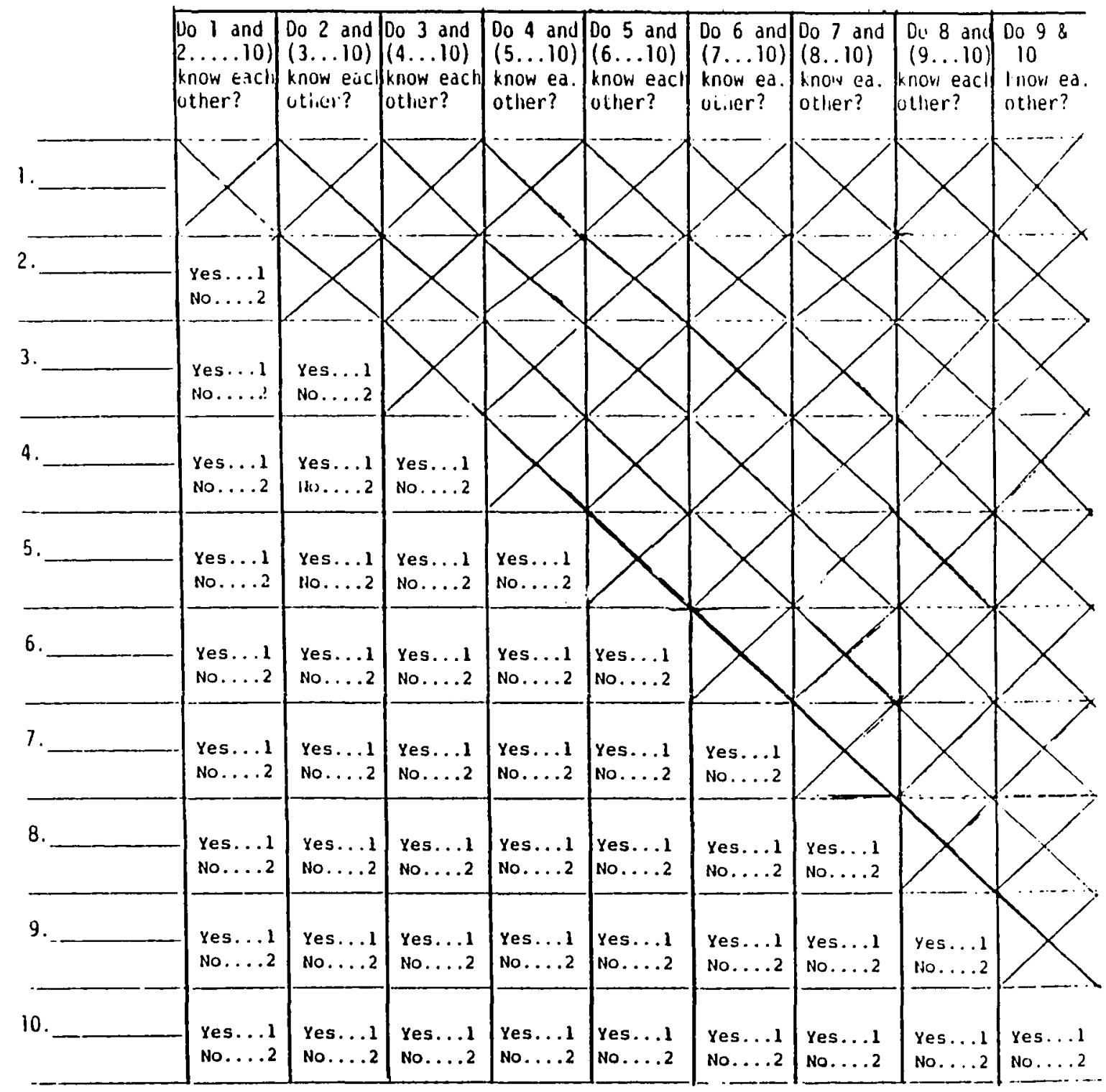

* INTERVIEWER ASK: Are there any of these acquaintances that have come about as a result of the stroke? (CIRCLE ALL SUCII RELATIONSIIIPS) 
Referring hospital code

Date of Stroke

Handedness :
$1=$ right
$2=1$ eft
$3=$ ambidextrous

Iype_of Stroke :

1 = heworrhagic

$2=$ thrombo-embol $1 \mathrm{c}$

3 = lacunar

( $s p \in c i f y)$
Name

iddress

Tele.
Language Baskgceund:

1 = primary language English

2 = flrst language other than English (specify)
Site of lesien:

$1=$ left cerebral hem 1 sphere

2 = right cerebral hem isphere

$3=$ brain stea

4 = other
Seyrce of Stroke-

Medical records

C.T. scan

Bra1n scan
Anglogram

Eac111ty Chronolegy:

Name

Admit Date

$D / C \quad D a t g$

Theraples Recelved: P.T. O.T. S.T. Other (specify)

curcent Therap1es:

P.T. O.T. S.T. other other Provider:

Therapy_codes :

Referr1ng Hospltal_codeg :

$0=n_{0}$

1 = Good Samaritan

$2=$ V.A.

$5=S t$. Vincent

$1=$ yes

$3=$ Emanuel

4 = Salem Mem. /General
$6=$ OHSU

$7=$ Providence 
APPENDIX B

FOLLOW-UP INTERVIEW SCHEDULES 
STRORE SURVEY: SOCIAL SUPPORT AND HELL-BEING

FOLLOH- OP INTERVIEH SCHEDULE:

PRIMARY INPORMANT/STROKE VICTIM

Place of Interviow

Interviewer

Date.

Interview time begin

Interviow time end

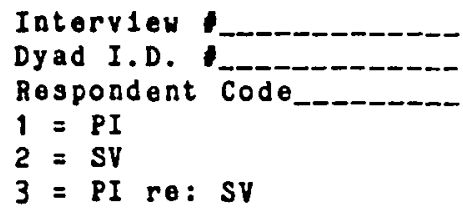

Pirst, let me thank you for taking the time to talk witb us again. Ho are very interested as you know in learning more about stroke patients and their samilies and friends. I again have a number of questions to ask you, so let we know if you need to rest, to take a break, or want furtbor explanation. Some of the questions w11l be similar to those we asked before. Let me reassure you tbat all Information you provide will be kept confidential. During this interview, we'll be interested 1n how things have been going for you in the past six months, since we last talked.

1. Has there been a change in your marital status in the last s1x months? (IF YES, HAND CARD TO R)

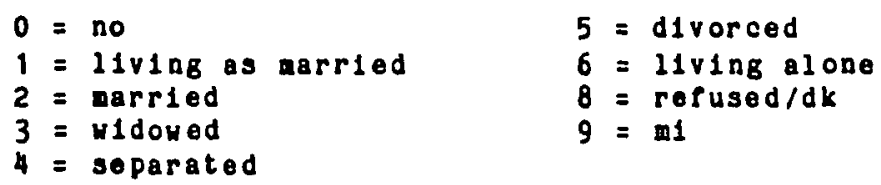

2. Have any of your cblldren moved in the last six wonths?
$0=$ no (sk1p to Q. 4)
$8=$ refused $/ d k$
$1=$ yes
$9=\mathrm{ml}$

3. May I please have the following information about your children?

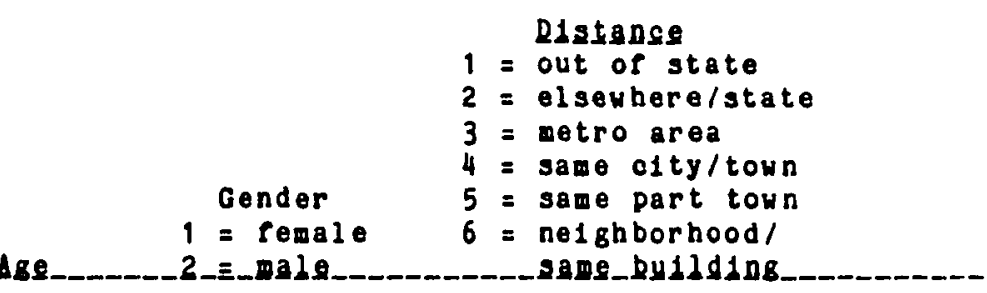

\section{Ch11d 1}

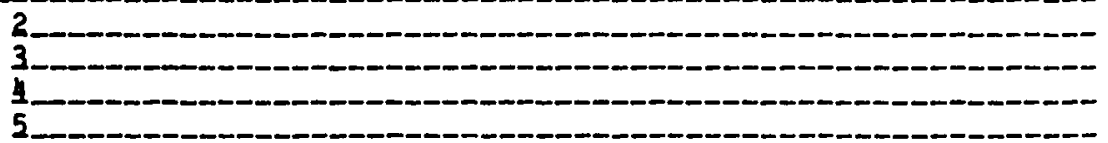

(Ose other side if $>5$ children.) 
4. Makeup of Household:

I'd like to ask some questions about the present makeup of your housebold, about tho people who live with you (and _-_.._. ). What are the first nawes of all the otber persons who live wich you on a regular basis? (LIST BELOW)

(If not obvious) Age Relat. to sv Miat. to PI

1. (Bespondent)

2.

3 .

4.

5 .

6.

7 .

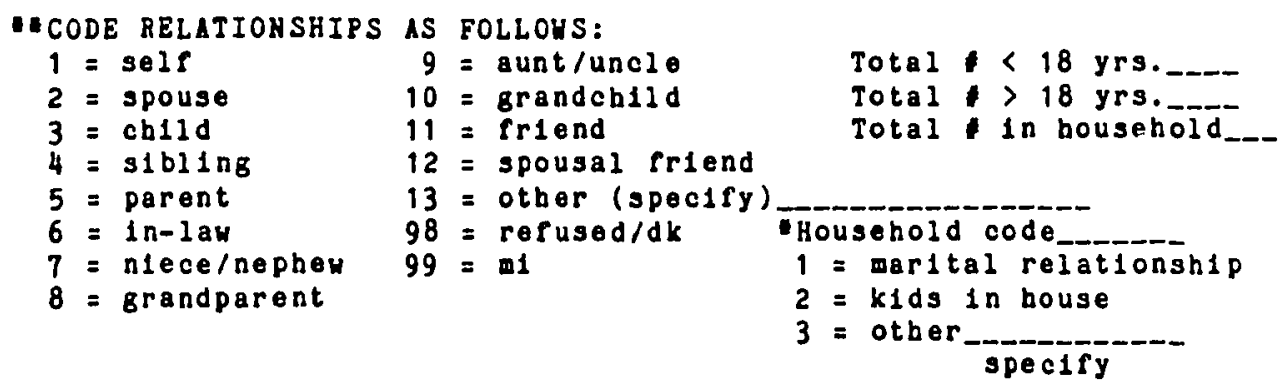

Now, I'd like to ask you some questions about your employment situation now.

5. What is your current employment status? (HAHD CARD TO R)

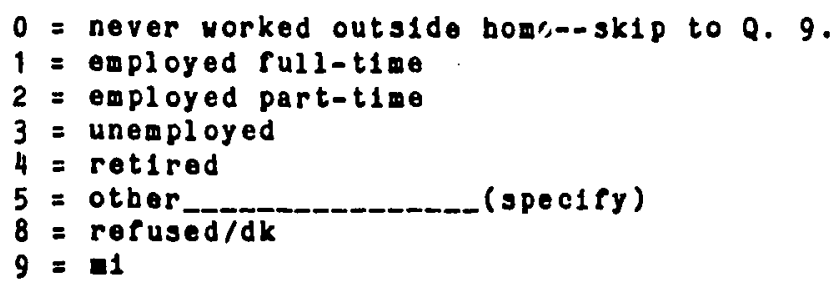


6. Is tbis a change frow six montbs ago?

$0=$ no (skip to Q. 9)

$1=$ yes

7. What is your occupation callod?

EQB__LSHER_1__2 :

8. In general, bow satisfied are you with being a $?$ (HAND CARD TO R)
1 = very dissatisfied
5 = very satisfied
2 = somewhat dissatisfied
$8=$ refused $/ d k$
$3=$ neutral
$9=m 1 / n a$

4 = somewhat satiseled

9. How much, if at all, has your standard of living (economically) changed since the stroke occurred? (HAND CARD TO R)

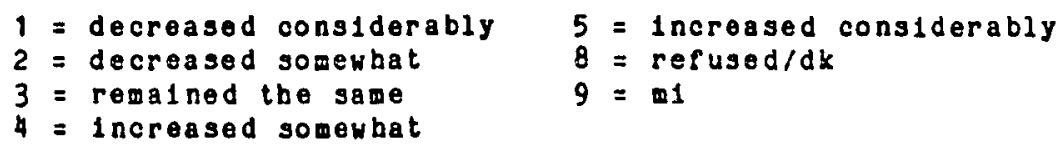

(ASK AT $\mathrm{T}_{3}$ ONLY)

10. In which of the following categories did your last year's total boysebeld lacome rall? (HAND CARD TO h)
$1=\langle \$ 5,000$
$2=\$ 5,000-\$ 9,999$
$3=\$ 10,000-\$ 14,999$
$4=\$ 15,000-\$ 19,999$
$5=\$ 20,000-\$ 24,999$
$6=\$ 25,000-\$ 29,999$

$$
\begin{aligned}
7 & =\$ 30,000-\$ 39,999 \\
8 & =\$ 40,000-\$ 49,999 \\
9 & =>\$ 50,000 \\
98 & =\text { refused/dk } \\
99 & =1
\end{aligned}
$$

11. Have you changed your residence in the last six montbs?

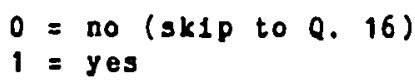

12. Could you tell we a litie about the clrcumstances that lead to your moving?

13. Residence setting? (INTERVIENER CODE)
$1=$ urban
$9=\Delta 1 /$ na
$2=$ rural
3 = suturban 
Fo110w-up Interview
5

Code

20. Lt the present, how active are you in your religious organization? (READ RESPONSES)
$1=\operatorname{lnact} 1 \mathrm{ve}$
$4=$ very active
2 = only a little active $8=$ refused/dk
3 = somewhat active
$9=\operatorname{mos}$

21. How often do you attend religlous services?
$0=$ never
$1=\langle 1$ month
$4=$ weekly
$2=$ monthly
$3=2-3$ t1mes a wonth
$5=>$ weekly
$8=\operatorname{refused} / d k$
$9=m 1$ 
22. What clubs, groups or other organizations do you presently belong to? (HAND CARD TO R)

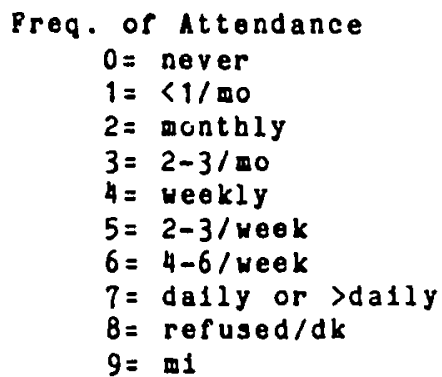

Frea.

Org_Hame

- organization code

$0=$ none

1 = business, prof or tech

2 = church connected

3 = charltable

$4=c 1 v 1 c$, communtty or ne1ghborbood

5 = rraternal or veterans

$6=$ pol1tical
7 = senior center

$8=$ social, sport or renreat 1 ona 1

$9=$ youth related

$10=$ other

$98=\operatorname{refused} / d x$

$99=m 1$

23. Do you belong to any self-belp or support groups?

$0=$ no

$1=\operatorname{yes}($ specify)

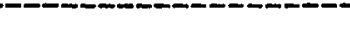


24. Do you attend any stroke club weetings?

$0=$ no (IF NO, ASX Q. 25)

$1=$ yes (IF YES, SKIP TOQ. 26)

25. Is there a stroke club avallable in your area?
$0=$ no
$8=\operatorname{refused} / d k$
$1=$ yes
$9=01$

26. How helprul do you feel that the stroke club bas been to you and ?
$1=$ not very helpful
2 = somewhat helpful
$8=\operatorname{refused} / d k$
3 = very helpful
$9=m i / n a$

27. In what ways have you found a stroke club helpful?

(spectfy)

-

Stroke club belp:

Code as 1 = effective/emotional

2 = instrumental

$3=$ informational

$4=$ combination

28. Do you give your time to volunteer work?

$0=$ no

$1=$ yes

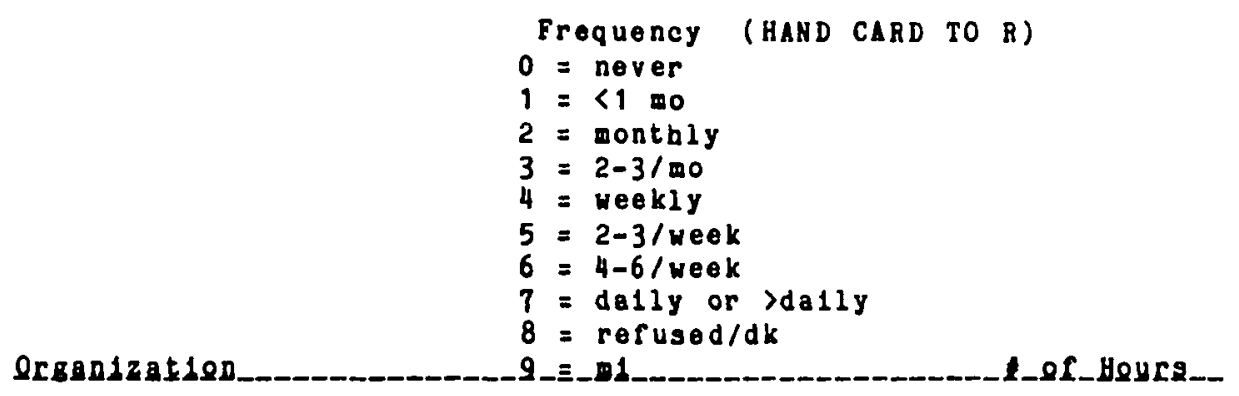


29. Have you found that there are some bobbles or special interests you are no longer ablo to pursue since tbe stroke?
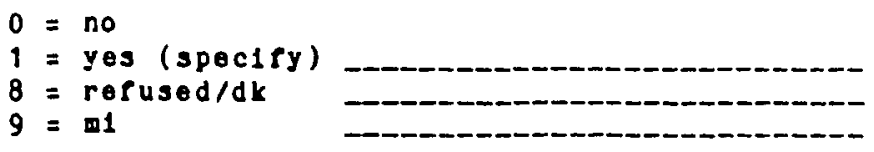

30. Have you sound there are some now hobbles or special interests you bave taken up since the stroke?
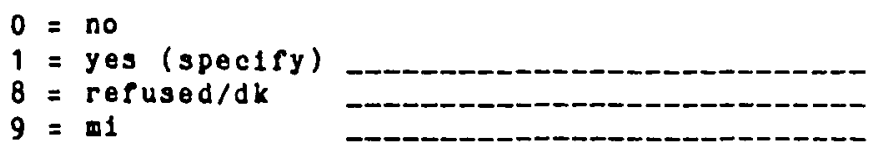

31. Have you used any community services and/or agencles in the past six months?

How belpful?

1 = not very helpful

2 = somewhat helpful

Serx1sg_Name 도요 3_E_yery_beleful

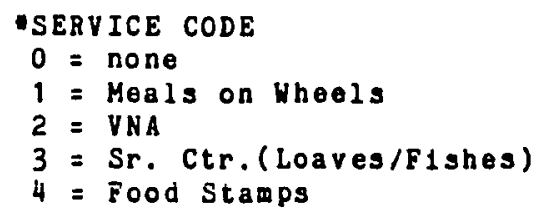

Total of Services

HEALTH/WELL-BEING :

In the following sootions are questions regarding your health and well-belng.

32. Have there been any 1eportant good or bad events that happened to you in the last six months? (FOR EXAMPLE: Death of someone close, birth of grandchlid, retirement, serlous accident, etc.)

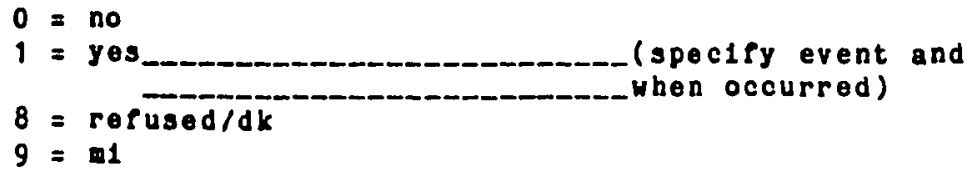


33. Do you currently have any physical disabilities that 1101 t your work or social activities? (ASK OF SV ONLY IF NOT OBVIOUS)

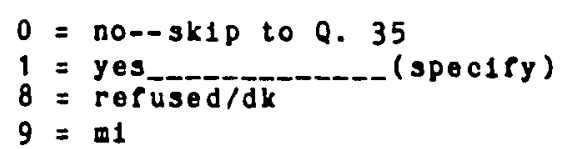

34. Does this _._._._._._. require the use of special services, alds or equipment? (PROBE: For exapple, braces, vision or hearing alds, hand ralls, bospltal beds, etc.) (ASK OF SV ONLY IF NOT OBVIOUS)

$$
\begin{array}{ll}
0=\text { no--1f no, probe } & 8=\text { refused } / \mathrm{dk} \\
1=\text { yes } & 9=\mathrm{m} 1 / \mathrm{na}
\end{array}
$$

35. How yould you rate your overall bealtb at the present time? (HAND CARD TO R)

$$
\begin{array}{ll}
1=\text { excellent } & 5=\text { poor } \\
2=\text { very good } & 8=\text { refused } / \mathrm{dk} \\
3=\text { good } & 9=\mathrm{ml} \\
4=\text { falr } &
\end{array}
$$

36. Is your bealth now better, about the same or worse than it was six months ago?

$$
\begin{array}{ll}
1=\text { better } & 8=\text { refused/dk } \\
2=\text { same } & 9=m 1 \\
3=\text { worse } &
\end{array}
$$

37. Do you feel that within the next six months your overall health is likely to (READ RESPONSES)

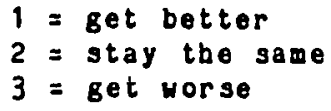

38. Do you have any chronic health problems? (IF NO ANSWER, PROBE: e.8., Diabetes, heart condition, high blood pressure)
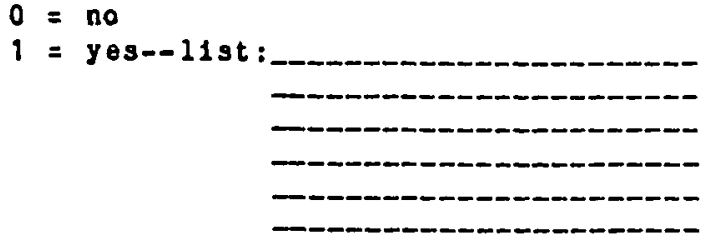

- Chronic Health Probs 
33. Have you been hospitalized in the last six wonths?

$0=$ no

1 = yes--bow wany times

$8=$ refused $/ d k$

$9=m 1$

$$
\text { lengtb of hospitalization (days) for each }
$$

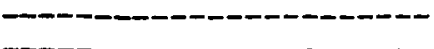

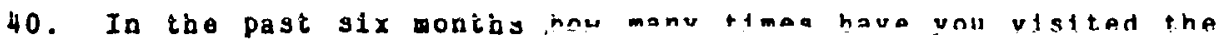
doctor?

Total

41. What medications are you now taking on a regular basis?
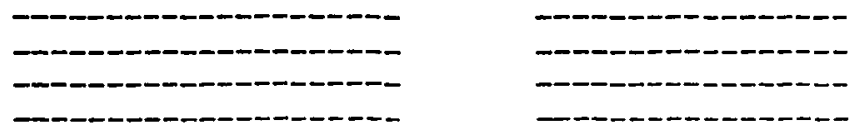

42. In the past six montbs haye you had an emotional problem for whlch you wanted belp?

$$
\begin{aligned}
& 0=\text { no } \quad 8=\text { refused } / \mathrm{dk} \\
& 1=\operatorname{yes}(\text { IF YES, ASK Q. 43) } 9=m 1
\end{aligned}
$$

43. Did you receive belp for this concern?

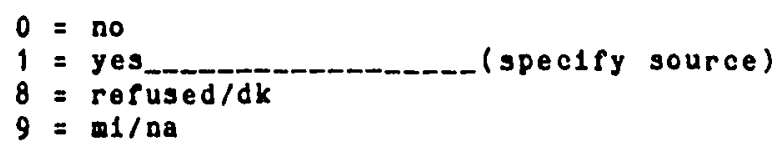

44. In the last $91 x$ wonths, have you experienced any change in your appetite?

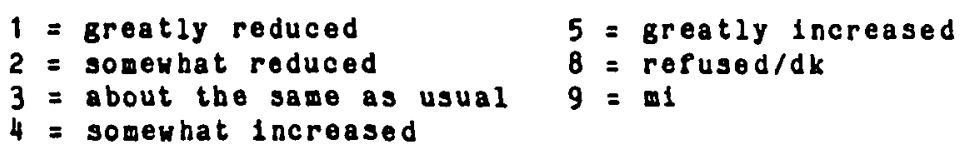


45. Have you experienced any change in your sloeping habits in the past six months?

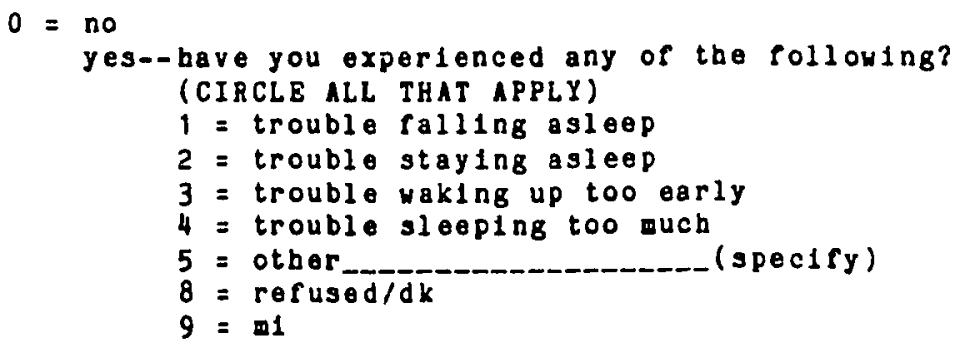

STRORE--PERCEPTIONS \& FEELINGS:

Now I'd like to ask some questions about the stroke.

46. Think1ng back, can you now think of anything you wight have done to pravent tho stroke?

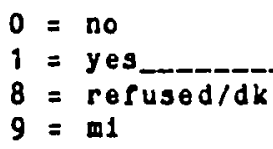

47. At this time, how concerned are you about the possibility of another stroke? (HAND CARD TO R)

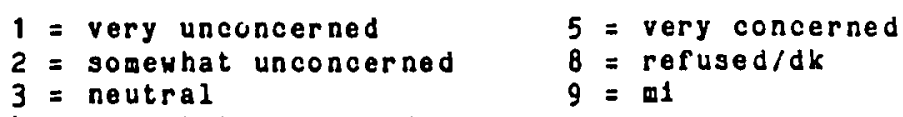

48. In your viey, bow severe are __._._. 's physical health problems nOW? (HAND CARD TO R) (REWORD AS APPROPRIATE FOR SV)

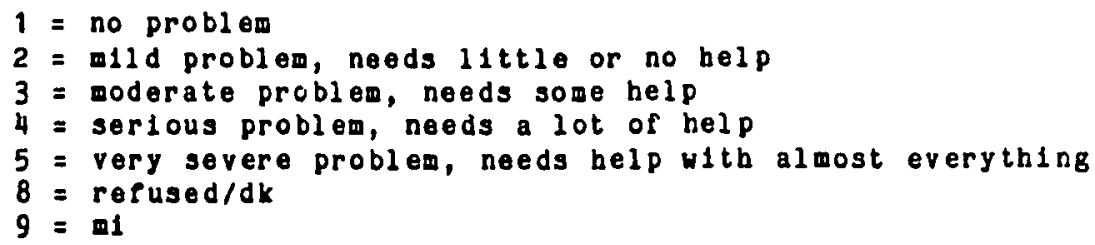

49. How concerned are you that you might not be able to care for SV) in the future? (HAND CARD TO R) (REHORD AS APPROPRIATE FOR

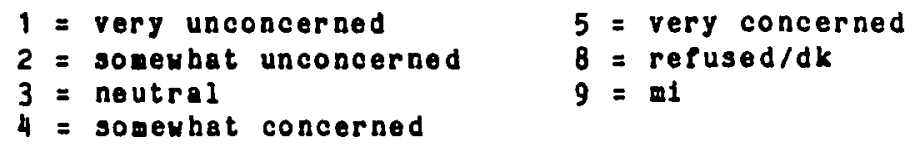


50. In the past six montbs, who or what has been your greatest sources of strength in helping you adjust to the stroke? (UP TO THREE SOURCES) (HAND CARD TO R)

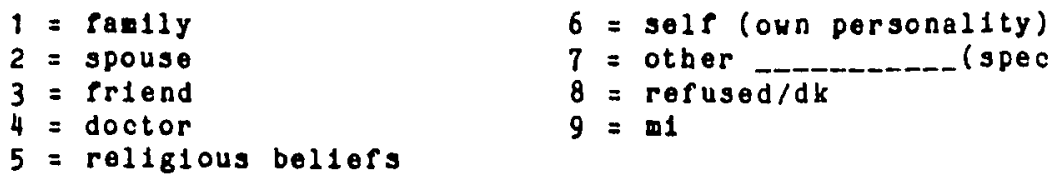

51. Overall, how satisfled have you been with the bealth care bas recelved for the stroke? (HAND CARD TO R) (REWORD AS APPROPRIATE FOR SV)

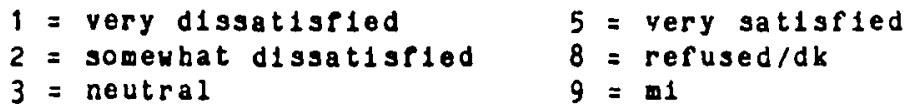

52. Prow the perspective of s1x months, who or what do you blame mest for the stroke happening?

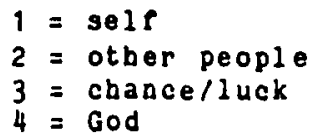

Now I want to ask a few questions about your relationship with --about some of the thlags you enfoy dolng together, and some of the ways you spend time together. Some of the following questions may or may not fit your rolationghip with $\ldots$ everyone has different ways of communicating. These next questions wil be about your relationship in the last six months.

53. In the last six months, how often bave you and In ectivities or outside interests together?

54. Had an interesting conversation about 1deas or oplnions?

55. Laughed togetber?

56. Calmly discussed sometblng?

57. Horked together on a project?

\section{(HAND CARD TO R)}

$$
\begin{aligned}
& 0=\text { never } \\
& 1=<1 \text { mo } \\
& 2=\text { monthly } \\
& 3=2-3 / \text { month } \\
& 4=\text { weekly } \\
& 5=2-3 / \text { week } \\
& 6=4-6 / \text { week } \\
& 7=\text { da1ly or }>\text { da1ly } \\
& 8=\text { refused/dk } \\
& 9=\text { mina }
\end{aligned}
$$


58. At present, do you and

participate in any of the same organtzations? (SPECIFY HAME AND CODE BY TYPE OF ORGANIZATION):

Name of Organization
- Trypo of Orlianization

- organization CODE
$0=$ none
- or organizat.

1 = business, prof or tech

2 = church connected

$3=$ charitable

$4=c 1 v 1 c$, comounity or nelghborhood

5 = fraternal or veterans

$6=$ pol1tical

7 = senior center

$8=$ social, sport or recreational

$9=$ youtb related

10 = otber (specify)

$98=$ refused/dk

$99=\$ 1 /$ na

59. At present, how often would the two of you be likely to disagree on the following:

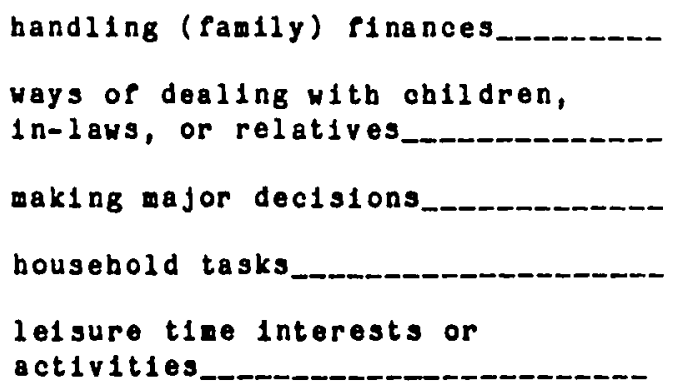

lelsure tine Interests or activities for :

60. At the present time, who holds the primary responsibility

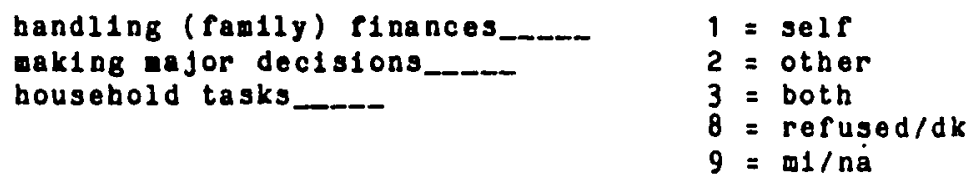


61. Think about your relationship with In the past $s 1 x$ wontbs. If you bad a very personal and serlous problem, how likely would you be to share it witb _._._? Would you say it was (HAND CARD TO R)

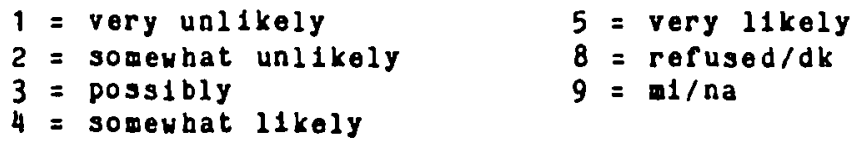

62. Igain, in the past six montbs, if _... had a very personal and serious problem, bow likely would be/she be to share it with you? Hould you say it was (HAND CARD TO R)

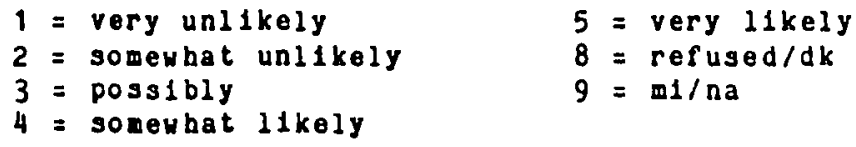

63. In general, how happy are you with your marriage (relationship) right nOy? (HAND CARD TO R)

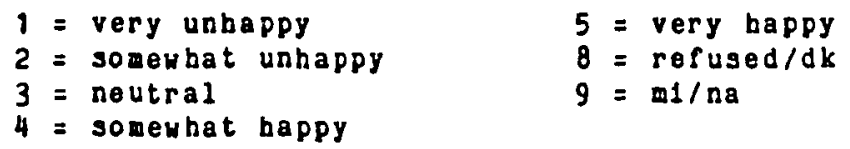

64. Compared to other couples you have known, overall, would you rate your marriage (relationship) as worse, about tbe same as, or better than other couples?

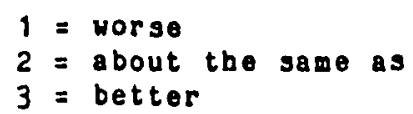


65. Some families and friogds bave observed personality and behavior changes in a person following a stroke, while other people see the stroke patient as very much the same in personallty and behavior as they were before. He would like to ask you some questions now about possible personality and bebavior changes in _-___... I'll read some words or phrases that doscribe tho ways people sometimes feel or act. I'd like you to tell me whether _._. behayes or feels like this less than before tho stroke, abeyt_tbe_same as beforo the stroke, or pere since the stroke. (HAND CARD TO R)

\section{Less About_tbesame Hore \\ (1) \\ (2) \\ (3)}

1. thoughtful (considerate)

2. angry (short-tempered)

3. 1rritable (easily upset)

4. restless

5. self-centered

6. anxious (fearful, nervous)

7. shows initiative (planning)

8. Impulsive, (uses poor Judgment)

9. laughs

10. cries

11. pat1ont (tolarant)

12. Indifferent, (18cks inttiative)

13. ploasant, oasy to get along with

14. susplc1ous

15. Jealous (possessive)

16. confused (perplexed)

17. depressed (sad)

18. happy (cheerful)

19. Porgetful (poor memory)
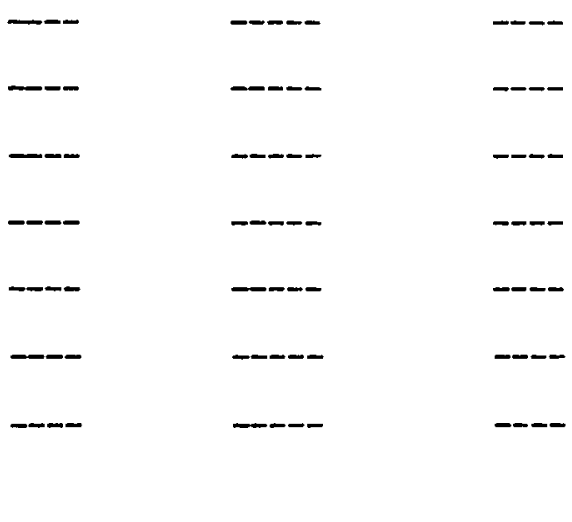
SOCIAL NETHORR-SOCIAL SUPPORT DATA:

Now I'd like to ask some questions about the people who have been toportant to you over the past few montbs. They may be famlly, frlends, nelgbbors, co-workers, or otbers. I want to get as accurate and complete a plcture as I can about tho people you've felt closest to, have spent tize with, have depended upon for belp, or who have depended on you for help.

66. In the past s1x montbs, about how often have you:

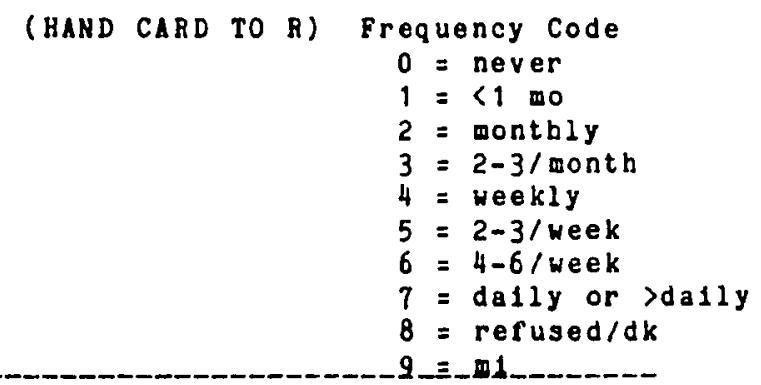

(1) Had someone to your home for lunch or dinner

(2) Gone to someone's home for lunch or dinner

(3) Had someone come by your house to visit

(4) Gone over to someone's house for a visit

(5) Gone out with someone (to a restaurant, movie, etc.)

67. If you needed someone to keep an eye on the house, take in a package, or look after a pet while you spent time away from your home, are there people you would ask to belp in thls way? (90)

$$
\begin{aligned}
& 0=\text { no } \\
& 1=\text { yes }
\end{aligned}
$$

Could you give me the pirst names of the people you would ask?

(IF YES, ASR FOR FIRST HAHES AND LAST INITIAL, AND RECORD ON NAME LIST. FOLLOW SAHE FORHAT AND RECORD NAMES FOR QUESTIONS 68-88.)

68. Durlng the last month or two, bave there been some people who've actually belped in this way, that 1s, kept an eye on the house, or belped with housebold tasks? (91) ways?

69. Are there people who would ask yoy to belp them in these 
70. Thlaking back over the past six gonths, do you bave any friends, nelghbors, family nembers or otbers wo would belp you if you were sick for a sbort tiae, that 1s, for a few days up to a week? (PROBE: prepare a meal, look in) (71)

71. How about people who would ask you to belp tbem if they were sick for a short time? Do you have any friends, nelghbors, or family wembers who would ask for your belp in thls way? (72)

72. When you are concerned about a personal matter, for example, about someone you are close to, or something you are worrled about, how often do you talk about it witb someone? Hould you say, (73)

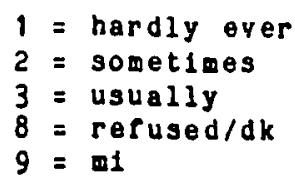

73. Think1ag about the last six montbs, when you have talked with someone about personal matters, whom have you talked with? (74)

74. Are there people who would come to you to talk about sowething they were worried about, or about a personal matter? Remember, it can be a ramlly member, friend, nelghbor, co-worker, or someone else. (75)

75. Orten, poople ask advice, or rely on the judgment of someone they know in making important decislons about thelr lives--for example, about thelr family or their work. Is there anyone whose opinion, in the past six nonths, you've considered seriously in mak1ng important dec1sions? (76)

76. Are there poople who would seek your oplnion or advice about things, or who bave in the last six months? (77)

77. Sometimes people get together with close friends, nelghbors, ramily or otbers to do something enjoyable togetber, or just to socialize. May I have the rirst naves (and last initial) of persons you'vo done (or do) those things with en_e_regylar_basis? (78) 
78. If you became 111 or neoded belp for longer than just a few days, say weeks or montbs, is thero someone, or more than one person you could call on to bolp, to come and stay witb you if needed, or take you into their home? (80)

79. If you needed a ride somewhere, to shop, visit, or get to an appointment, for example, are thore people you could call on to help? (88)

80. During the last month or two, bave there been some people who've actually belped in this way, that 1s, provided transportation belp? ( 89$)$

81. Are there people who've been to visit you in the last month or two to offer you encouragement and "moral support"? (92)

82. Lbout how wany people in the past month or two have called to find out bow you and (SV or PI) were doing? (93)

83. Lre there people who've been belpful in otber ways in the last couple of months, for example, who've come to stay with you, had you stay with them, offered to run erands for you, or to give you a break from dally chores? (94)

84. Sometimes friends, fam1ly, nolghbors, or others can be heipiul in providing information or advice when we need $1 \mathrm{t}$. are there any people wholve given you belprul information or advice in the last couple of months about stroke, ways to deal with a stroke, or about resources to help stroke patients and their families? (95)

85. Are there any people you know who appreciate or understand what you are going through right now as the _.... of soweone who has bad a stroke (OR, FOR SV, as someone who has suffered a stroke?) (96)

86. Are there any otber ways in whicb people bave helped, or been there to provide support in the last month or two? What would they be? Who bas provided tbis holp? (ADD NAMES TO NAME LIST, AND NOTE AND CATEGORIZE TYPE OF HELP, IF ABLE) (97) 
87. Thlaking about the last couple of months, bave there been people who bave belped core than you expected, or who have been supportive when you didn't expect thom to belp at all? (98)

88. (PRESENT NAME LIST TO RESPONDENT TO VIEW)

Thinking about the last couple of wonths, are there any otber people wo are iaportant to you wose names are not on this list? Remember they can be famlly, friends, nelghbors, co-workers, or members of an organization you belong to. (99) (CATEGORIZE TYPE OF HELP IF ABLE)

(ADD ANY ADDITIONAL MAMES TO NAME LIST)

SOCIAL NETWORK DATA - NEGATIVE ASPECTS:

In situations like this, sometimes we rind that there are people who don't help as wuch as we expect them to, or who don't help at all when we expected them to.

89. Lre there people who have helped durlag these past couple of montbs, but not as much as you expected they would, or who baven't helped at all when you thought they would?

May I have their first names? (RECORD NAMES AND OTHER INFORMATION BELOW )

Relationshlp Helped less

- Less belp tban expected

or no belp when expected. 
Follow-up Interview

20

Code

90. He all know people who try to belp, but end up making the situation worse. Are there any people you reel have been a hindrance to _...'s recovery thus far even though they try to be belprul?

$0=$ no

$1=$ yes

What are the1r names? (RECORD MAMBS AND OTHER INFO BELOW)

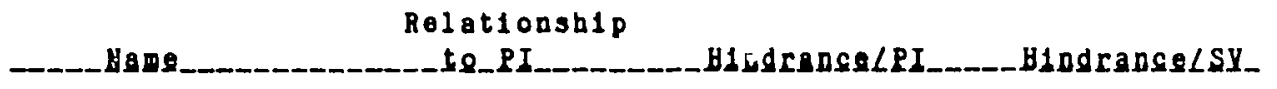

- Hindrance.

91. Sometimes we run into people who seem to be out to make IIfe difficult. Are there people like that who bave made problems for you in the last couple of months?

$$
\begin{aligned}
& 0=\text { no } \\
& 1=\text { yes }
\end{aligned}
$$

Who wOUld they be? (RECORD NAMES, RELATIONSHIPS BELOW)
Belat1203h1D_t2_PI 
92. We all know people we find lt oasy to get along with, and others we don't get along with. lre there people you don't get along with right now?

$$
\begin{aligned}
& 0=\text { no } \\
& 1=\text { yes }
\end{aligned}
$$

May I have their first names? (RECORD NAMES AND RELATIONSHIPS BELOW) Belat10n2b10_to_PI

- Don't get along with Total Negative

INSTRUCTIONS FOA COMPLETING NAME LIST, CODING SHEETS, AND MATRIX:

1. FOR ALL PERSONS ON NAME LIST, RECORD INFORHATION REGARDING RELATIONSHIP TO RESPONDENT, AGE, SEX, AND CLOSENESS (1ndicating 10 closest to).

2. ON CODING SHEET, RECORD NAMES OF UP TO 10 PERSONS IN APPROPRIATE BOXES LCROSS THE TOP, BASED UPON THE FOLLOHING CRITERIA :
a. THOSE HHOM RESPONDENT INDICATES THEY (OR SV) ARE ESPECIALLY CLOSE TO.
b. THOSE RESPONDENT INDICATES AS MOST IMPORIANT PROVIDERS OF SUPPORT, IF NOT LLREADY SELECTED.

3. IF 10 OR EEUER NW HEMBERS, $A S K$ QUESTIONS ON CODING SHEET ABOUT ILL OF THOSE MAMED.

4. COMPLETE IHFORHATIOH OH CODIHG SHEETS, USIHG APPROPRIATE SHEETS FOR PI AND SV.

5. CIRCLE MAMES THAT APPEAR OH BOTH SV AND PI CODING SHEETS.

6. COMPLETE INFORMATION ON HW MATRIX SHEET. 
93. In Beneral, bow satisfled are you with the smoynt of social contact you have with others at th1s time? (HAND CARD TO R)

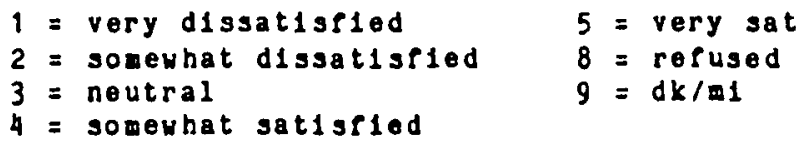

94. In general, how satisfied are you witb the quality or closeness or the social contact you bave with others at this time? (

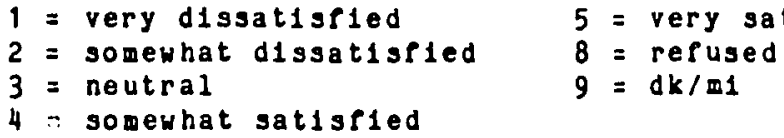

95. What is your greatest worry or concern at this time, related to the stroke?

96. Over the past few months, what aspects of the stroke have been most difficult for you to deal with?

97. What types of 1nformation, services, or assistance would have been belpful to you over the past few wonths? (PROMPT: In dealing witb the stroke) 
Follow-up Interview

98. What services or otber types of assistance would be helpful to you noy?
23

Code

- - - - - 


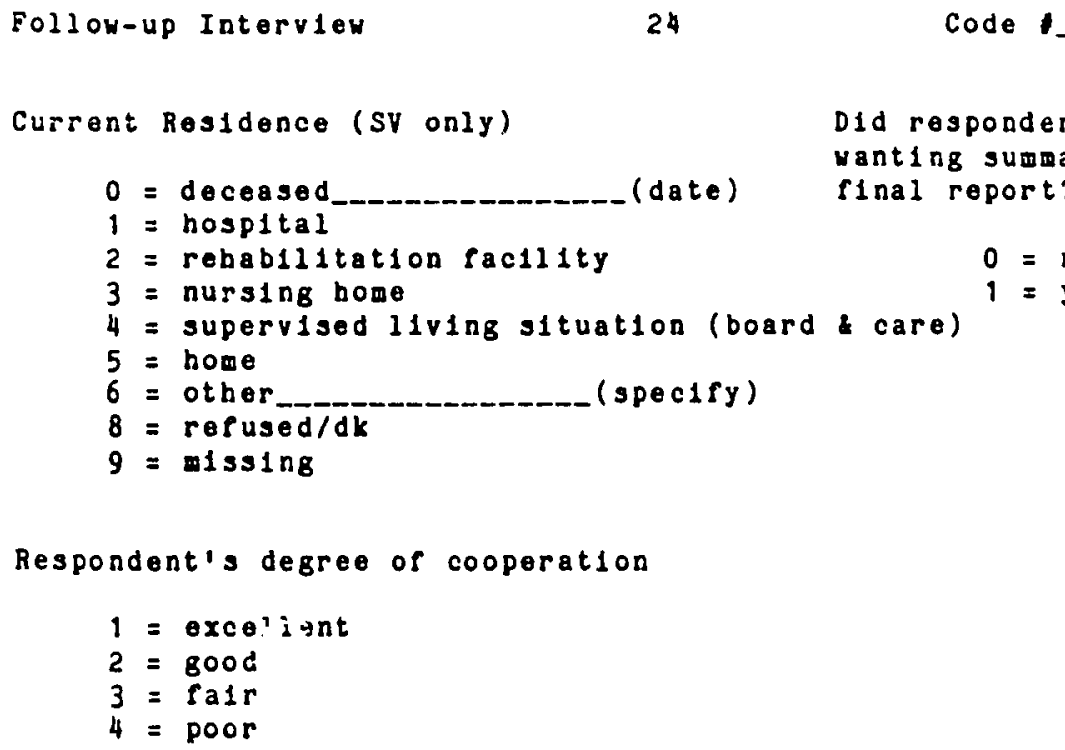

Was anyone else present during this interview?

$0=$ no

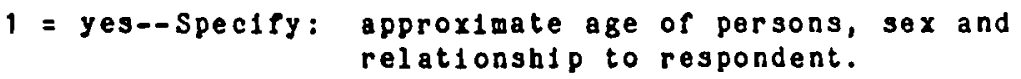

How long was this person present?

D1d this person participate in interview responses?

How well do you feel the respondent understood the ltems of this interview?

1 = understood all items with no trouble

2 = understood most 1tens, but had trouble with a few

3 = understood very few items, had troublewith most

4 = had trouble understanding all items

Note below any other unusual problems in this interview 


\section{STROKE SURVEY: SOCIAL SUPPORT AND NELL-BEING}

FOLLOW-UP INTERVIEW SCHEDULE: PRIMARY INFORHANT FOR STROKE VICTIM

Place of Interview

Interviewer

Date

Interview tioe begin

Interviow time end

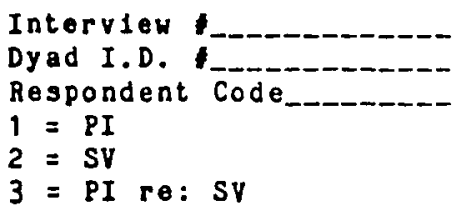

Interview

Dyad I.D.

$2=S$

$3=P I$ re: SV

I have a number of questions to ask you now about _...., so let we know if you need to rest, to take a break, or want further explanation. Some of the questions will be similar to those we asked before. Let me again reassure you tbat all information you provide W11 be kept confidential. We'll be interested in how things have been going for in the past six montbs, since we last talked.

(ASK Q. 1-4 ONLY IF NOT ALREADY DETERHINED)

1. Has there been a change in last s1x months? (IF YES, HAND CARD TO R)

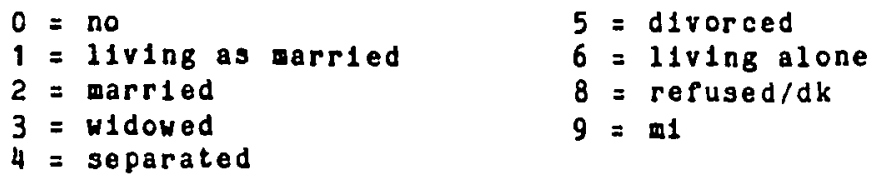

2. Have any of 's ch1ldren woved in the last gix wonths?
$0=$ no (skip to Q. 4 )
$8=$ refused $/ d k$
$1=$ yes
$9=\mathrm{mi}$

3. May I please have the following information about 's children?

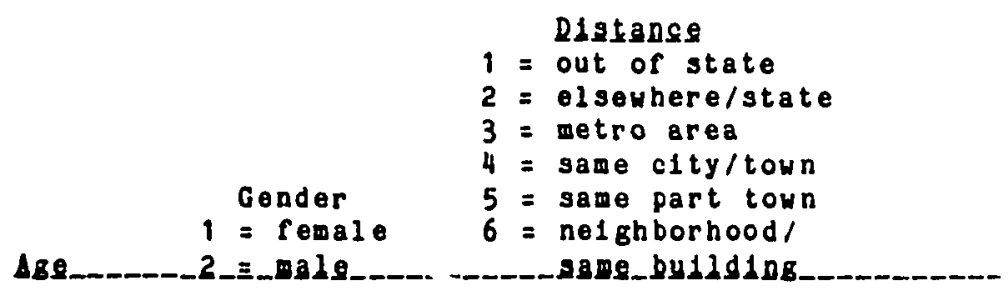

Ch119_1

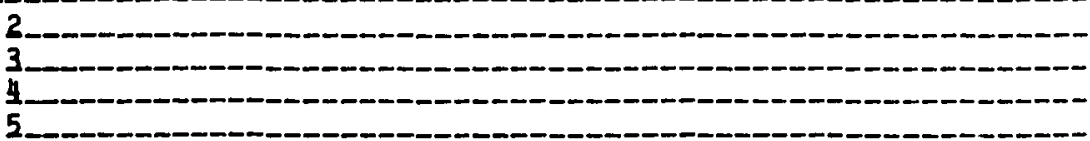

(Use other side if $>5$ chlldren.) 
4. Makeup of Household:

I'd like to ask some questions about the present makeup of - 's housebold, about the people who live with him/her. What are the flrst names of all the other persons who live with _... on a regular basis? (LIST BELON)

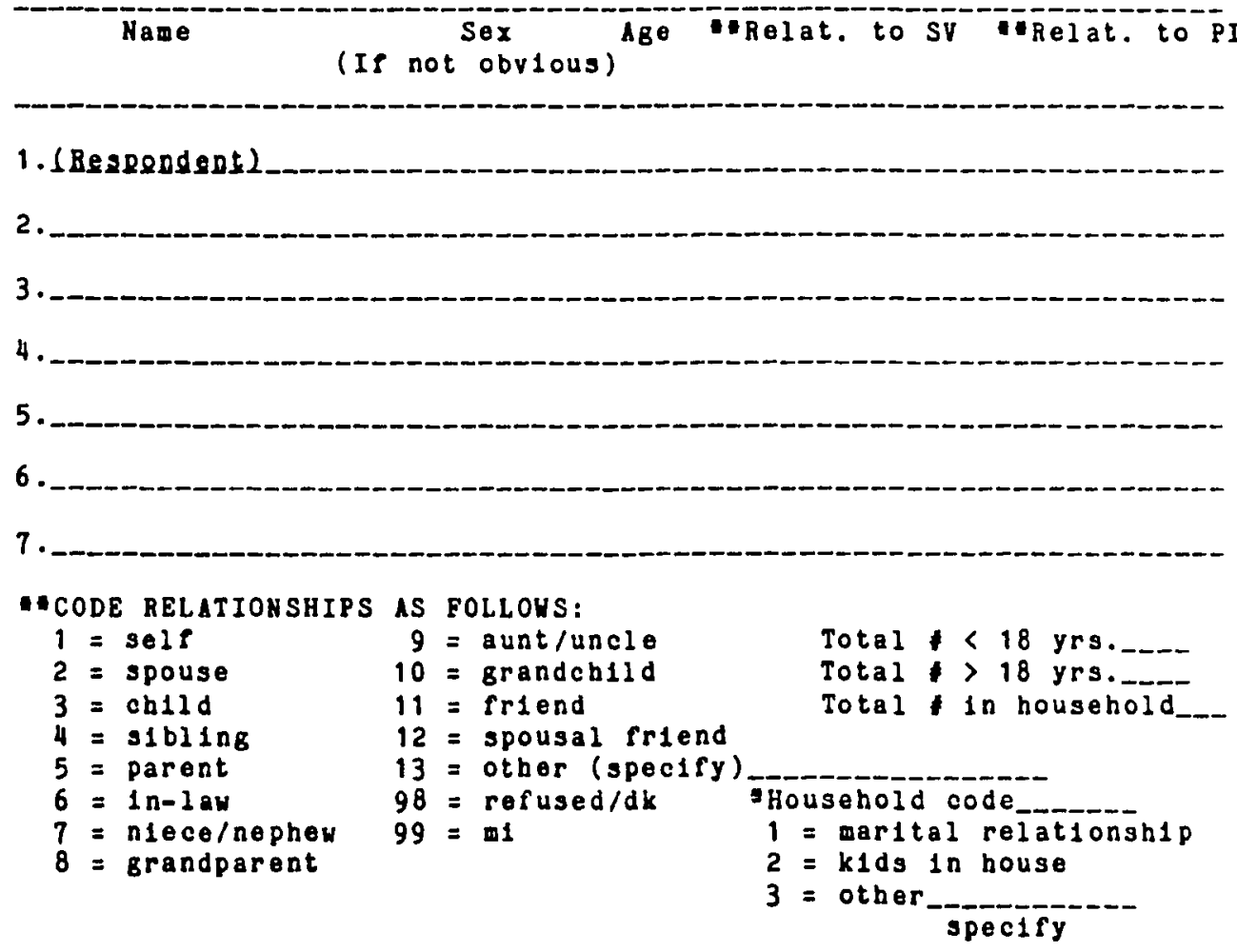

Now, I'd like to ask you some questions about _____'s employment situation now.

5. What is _-_-_'s current employment status? (HAND CARD TO R)

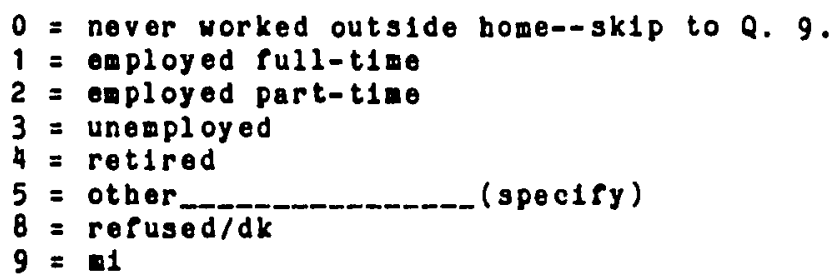


6. Is this a change frow s1x months ago?

$$
\begin{aligned}
& 0=\text { no (skip to Q. 9) } \\
& 1=\text { yes }
\end{aligned}
$$

7. What is 's occupation called?

(ASK Q. 9-15 ONLY IF NOT ALREADY DETERMINED)

9. How much, if at all, has _-_._'s standard of living (economically) changed since the stroke occurred? (HAND CARD TO R)

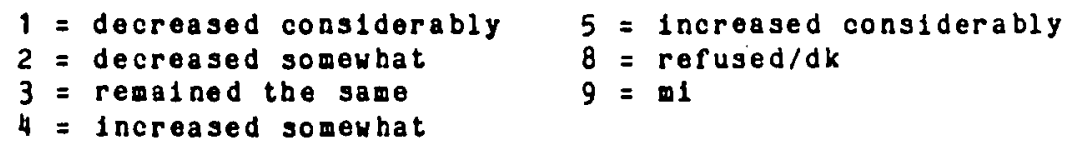

10. In which of the following categorles did__-_'s last year's total boysebold income fall? (HAND CARD TO R)

$$
\begin{array}{ll}
1=\$ 5,000 & 7=\$ 30,000-\$ 39,999 \\
2=\$ 5,000-\$ 9,999 & 8=\$ 40,000-\$ 49,999 \\
3=\$ 10,000-\$ 14,999 & 9=\$ 50,000 \\
4=\$ 15,000-\$ 19,999 & 98=\text { refused } / \mathrm{dk} \\
5=\$ 20,000-\$ 24,999 & 99=\$ 1 \\
6=\$ 25,000-\$ 29,999 &
\end{array}
$$

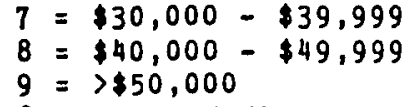

11. Has ___ changed b1s/her residence in the last six months?

$0=$ no (sk1p to Q. 16)

$1=$ yes

12. Could you tell we a little about the clrcumstances that lead to _.._'s moving?

13. Residence setting? (INTERVIENER CODE)
1 = urban
$9=m 1 / \mathrm{n} \theta$
2 = rural
3 = suburban

14. In what type or residence does I1ve?

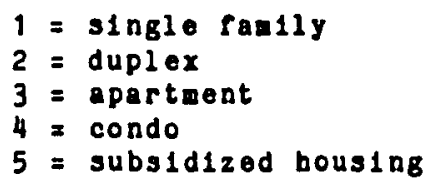


22. What clubs, groups or other organizations does s/he presertly belong to? (HaND CARD TO R)

Freq. of Attendance

$0=$ never

$1=\langle 1 /$ mo

$2=$ montbly

$3=2-3 / \mathrm{mo}$

$4=$ weekly

$5=2-3 /$ week

$6=4-6 /$ week

$7=d a 1 l y$ or $>d a 1 l y$

$8=$ refused/dk

$9=m 1$

Freq.

\section{- organization CODE}

$0=$ none

$1=$ business, prof or tech

2 = church connected

3 = charltable

$4=$ c1v10, comounity or ne1gbborbood

5 = rraternal or veterans

$6=$ poldtical

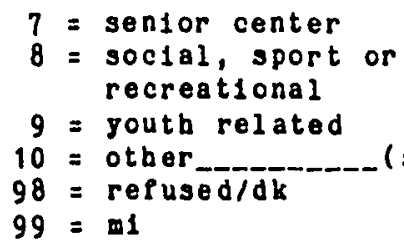

23. Does belong to any self-belp or support groups?

$0=$ no

$1=\operatorname{yes}(\operatorname{spec} f f y)$ 
Follow-up Interviow

24. Doos ___ attond any stroke club neetings?

$0=$ no (IF HO, AST Q. 25)

$1=$ yes

25. Is there a stroke club avallable in b1s/ber area?

$0=$ no

$8=\operatorname{refused} / d k$

$1=$ yes

$9=\mathbf{m}$

28. Doos give his/her tioe to volunteer work?

$0=$ no

$1=y e s$

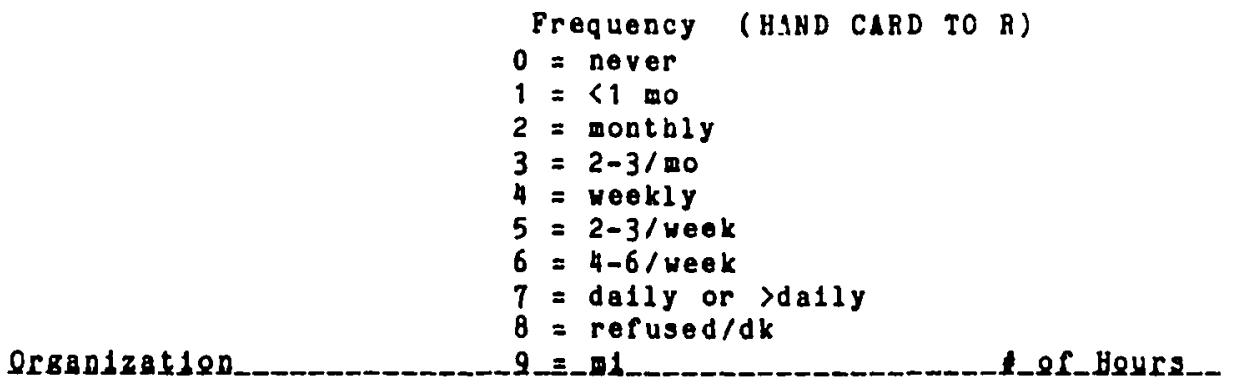

29. Have you found that there are some bobbles or special Interests is no longer able to pursue since the stroke?
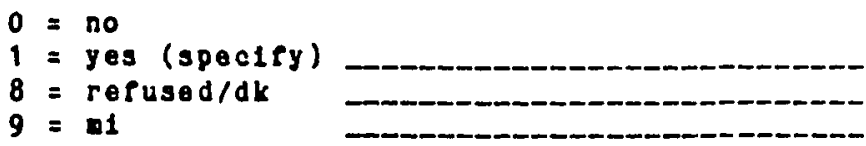

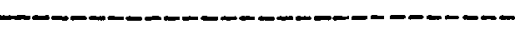

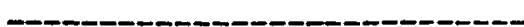

30. Have you found there are some new hobbles or special interests __ bag taken up alnoe tho stroke?
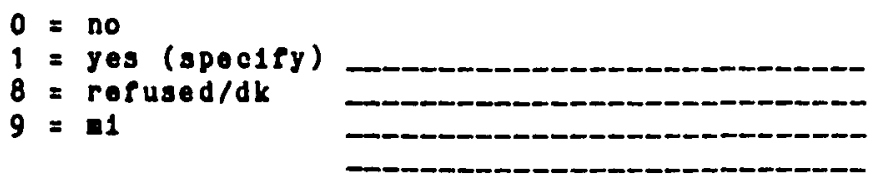
31. Has used any commulty services and/or agencies in the past six months?

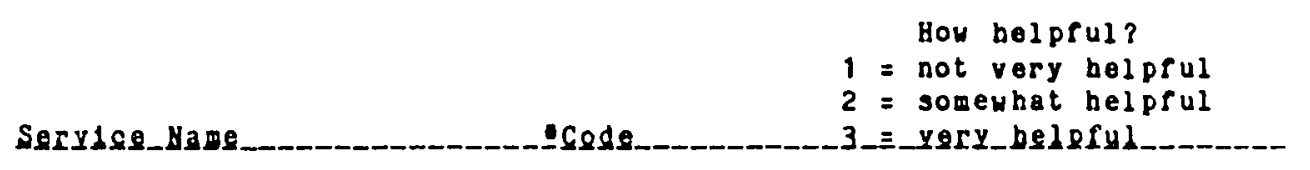

- SERVICE CODE

$\begin{array}{ll}0=\text { none } & 5=\text { Adult \& Fam. Serv. } \\ 1=\text { Heals on Wheels } & 6=\text { other (specify) } \\ 2=\text { VNA } & 8=\text { refused/dk } \\ 3=\text { Sr. Ctr. (Loaves/Fishes) } & 9=\text { m1 } \\ 4=\text { Food Stamps } & \end{array}$

Total of Seryices

HEALTH/HELL-BEING :

In the following sections are questions regarding and well-being.

32. Have there been any 1mportant good or bad events that bappened to _._ in the last six months? (FOR ExAMPLE: Deatb of someone close, birth of grandchild, retiresent, serious accident, etc.)

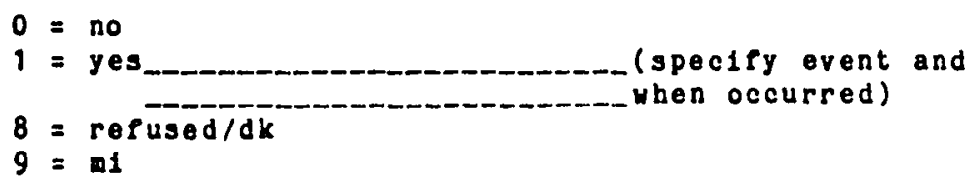

33. Does _-_- currently have any physical disabilities that 11 at his/ber work or social activities? (ASK ONLY IF NOT OBVIOUS)

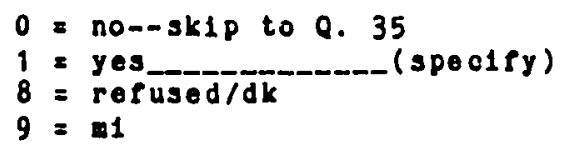

34. Does th1s ______ require the use of special services, alds or equipment? (PROBE: For example, braces, vision or bearing alds, band ralls, bospltal beds, etc.) (ASK OF SV ONLY IF HOT OBVIOUS)

$$
\begin{array}{ll}
0=\text { no--1f no, probe } & 8=\text { refused } / \mathrm{dk} \\
1=\text { yes_act } & 9=\mathrm{n}=1
\end{array}
$$


Follow-up Interview

35. How wotid you rate tIMO? (HAND CARD TO R)

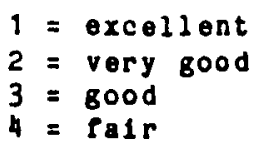

38. Does _-_ bave any chronic healtb problems? (IF NO ANSWER, PROBE: $0.8 .$, Diabetes, heart condition, bigh blood pressure)

$0=$ no

1 = yes--11st:

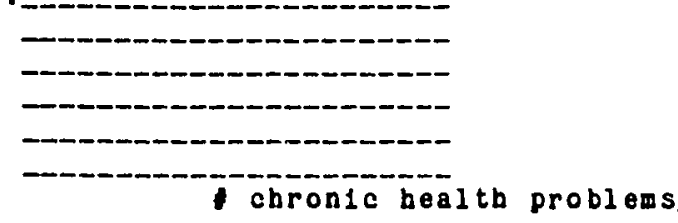

39. Has been hospltalized in the last six months?

$0=$ no

$1=$ yes--bow many times

$8=$ refused $/ d k$

$9=\Delta 1$
$5=$ poor

$8=$ refused/dk

$9=n$
8

Code 's overall bealth at the present

40. In the past $11 x$ months how wany times has visted the doctor?

Total

41. What medications is now taking on a regular basis?

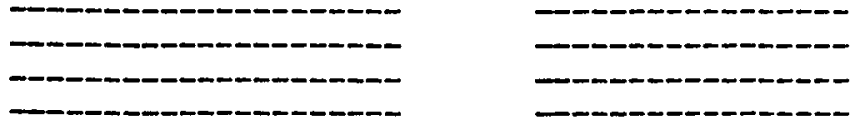

42. In the past six months has _-_. had an exotional problem for which he/she sought belp?
$0=$ no
$B=\operatorname{refused} / d k$
$1=$ yes (IF IES, ASR Q. 43) $9=$ m1

43. D1d recelve belp for this concern?

$0=$ no

$1=y 00$ (specify source)

$B=$ refused/dk

$9=1$ 
Code

44. In the last $31 x$ moatbs, bas experienced any change in his/ber appotite?

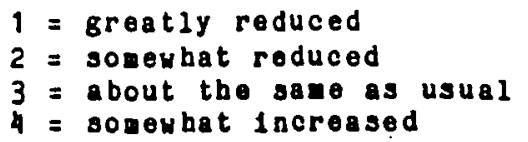

45. Has experienced any change in bis/ber sleepling habits in the past six aontbs?

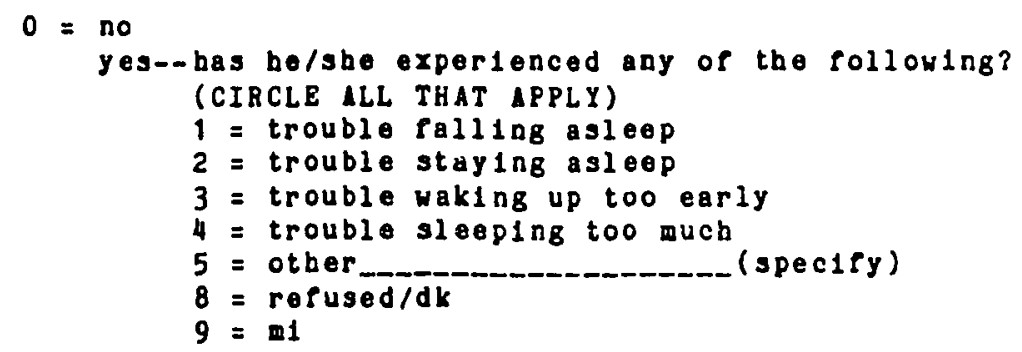

STROXE-PERCEPTIONS \& FBELINGS:

Now I'd like to ask some questions about the stroke.

46. Th1aking beck, oan you now think of anything _-_ might baye done to prevent tho stroke?

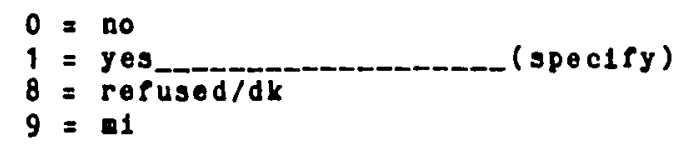

47. At th1s t1me, how concerned do you think _... is about the possibllity of anotber stroke? (HAND CARD TO R)

$\begin{array}{ll}1=\text { very unconcerned } & 5=\text { very concerned } \\ 2=\text { sonewhat unconcerned } & 8=\text { refused/dk } \\ 3=\text { neutral } & 9=m 1 \\ 4=\text { sorewhat concerned } & \end{array}$

SOCIAL HETWORK-SOCIAL SUPPORT DATA:

Moy I'd like to ask some questions about the poople who have been 1mportant to _._. over the past six months. They may be fan1ly, frleads, nelghbors, co-workers, or others. I want to get as acourate and copplete a ploture as I can about tho people _-_. has felt closest to, has spent time with, bas depended upon for belp, or who have depended on ____ for belp. 
Follow-up Intorviow

66. In the past s1x nontbs, about how often bes _-_-_:

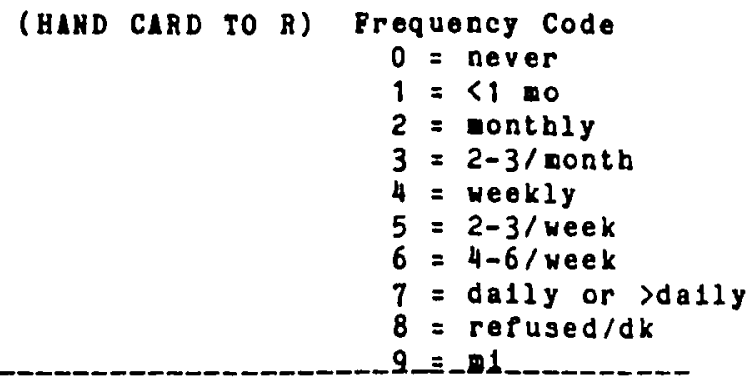

(1) Had someone to his/ber home for lunch or dinner

(2) Gone to someone's bowe for lunch or dinner

(3) Had someone cone by b1s/ber house to visit

(4) Gone over to someone's house for a visit

(5) Gone out with someone (to a restaurant, movie, etc.)

67. Over the past $s 1 x$ nontbs, are there people regularly speat tiae socializing with, visitiog, sharing hobbies or interests?

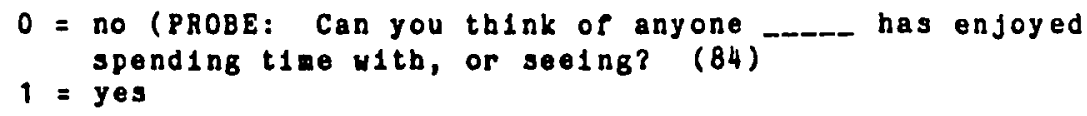

Hay I bave the rirst names and last initials of these people? (RECORD HAMES OY NAME LIST 4 . ROLLON SAME PROCEDURE FOR Q.'S 68-70)

68. It present, if _._. bad serlous concern or problem, to whom would be/sbe express this conoern, or talk to? (85)

69. In the last couple of months, who are some poople who've beon belpful to _....., for exapple, providing rides, helping bim/her wth tasks, staying witb _-_-, providing oncouragement and moral support, or belpful informetion, etc.? (100) 
70. (PRESENT NAME LIST 1 )

Are there any otber people who bave been laportant to over the past few montbs whose names are not on this list? Remember, they can be faplly, friends, nelghbors, co-workers, or members of the same organization. (86) (ADD NAMES TO NAME LIST A, AND CATEGORIZE TYPE OF HELP IF ABLE)

71. Aro there people _-_ doesn't got along w1th now? Who would they be? (87)

INSTRUCTIONS FOR COHPLETING NAME LIST, CODING SHEETS, AND MATRIX:

1. FOR ALL PERSONS ON hAME LIST, RECORD INFORMATION REGARDING RELATIONSHIP TO RESPONDENT, AGE, SEX, AND CLOSENESS (Indicating 10 olosest to).

2. ON CODING SHEET, RECORD NAMES OF UP TO 10 PERSONS IN APPROPRIATE BOXES ACROSS THE TOP, BASED UPON THE FOLLOHING CRITERIA :
a. THOSE HHOM RESPONDENT INDICATES THEY (OR SV) ARE ESPECIALLY CLOSE TO.
b. THOSE RESPONDENT INDICATES AS MOST IHPORTANT PROVIDERS OF SUPPORT, IF HOT ALREADY SELECTED.

3. IF 10 OR EEWER HW MEMBERS, ASK QOESTIONS ON CODING SHEET ABOUT ILL OF THOSE HAHED.
4. COMPLETE IHFORMATION ON CODING SHEETS, USING APPROPRIATE SHEETS FOR PI AND SV.

5. CIRCLE MAKES THAT IPPEAR ON BOTH SY AND PI CODING SHEETS.

6. INDICATE PI AKD SV ON MAME LISTS AND CODING SHEETS WITH (PI) OR ( SV).

7. COMPLETB IMFORMATION ON MH MATRIX SHEET. 


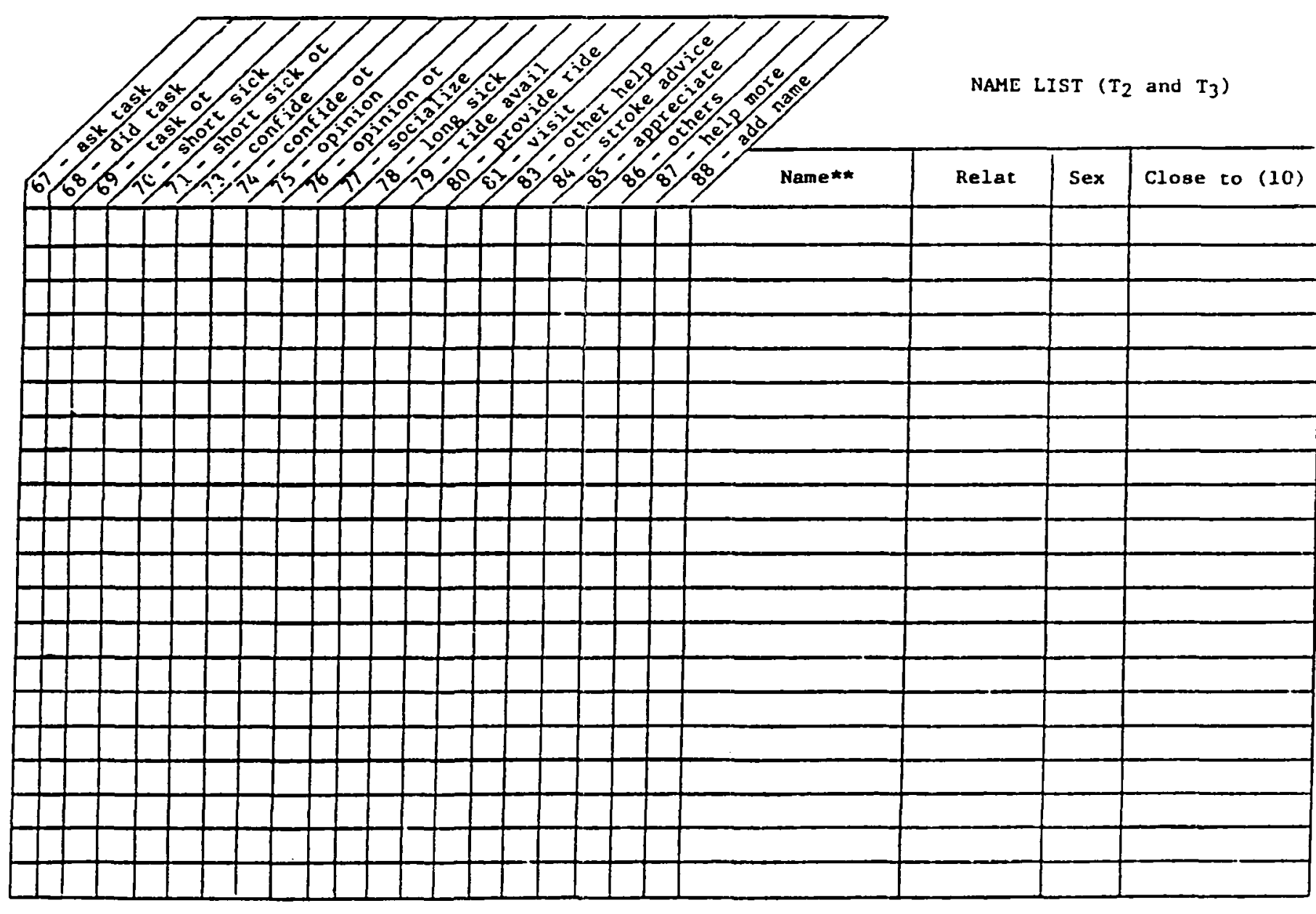

I.D.

NAME LIST $\left(T_{2}\right.$ and $\left.T_{3}\right)$

Relationship Code 0-ocher

1-spouse

2-child

3-sibling

4 -parent

5-other relative

6-neighbor

7-work associate

8-friend

Sex Code

$1-f$ enale

$2-$ male

Indicate $P I$ and $S V$ names:

* Cenerate a maximum of 20 nanes with relationship and sex information on each (first name and last initial) 
I.D. No.

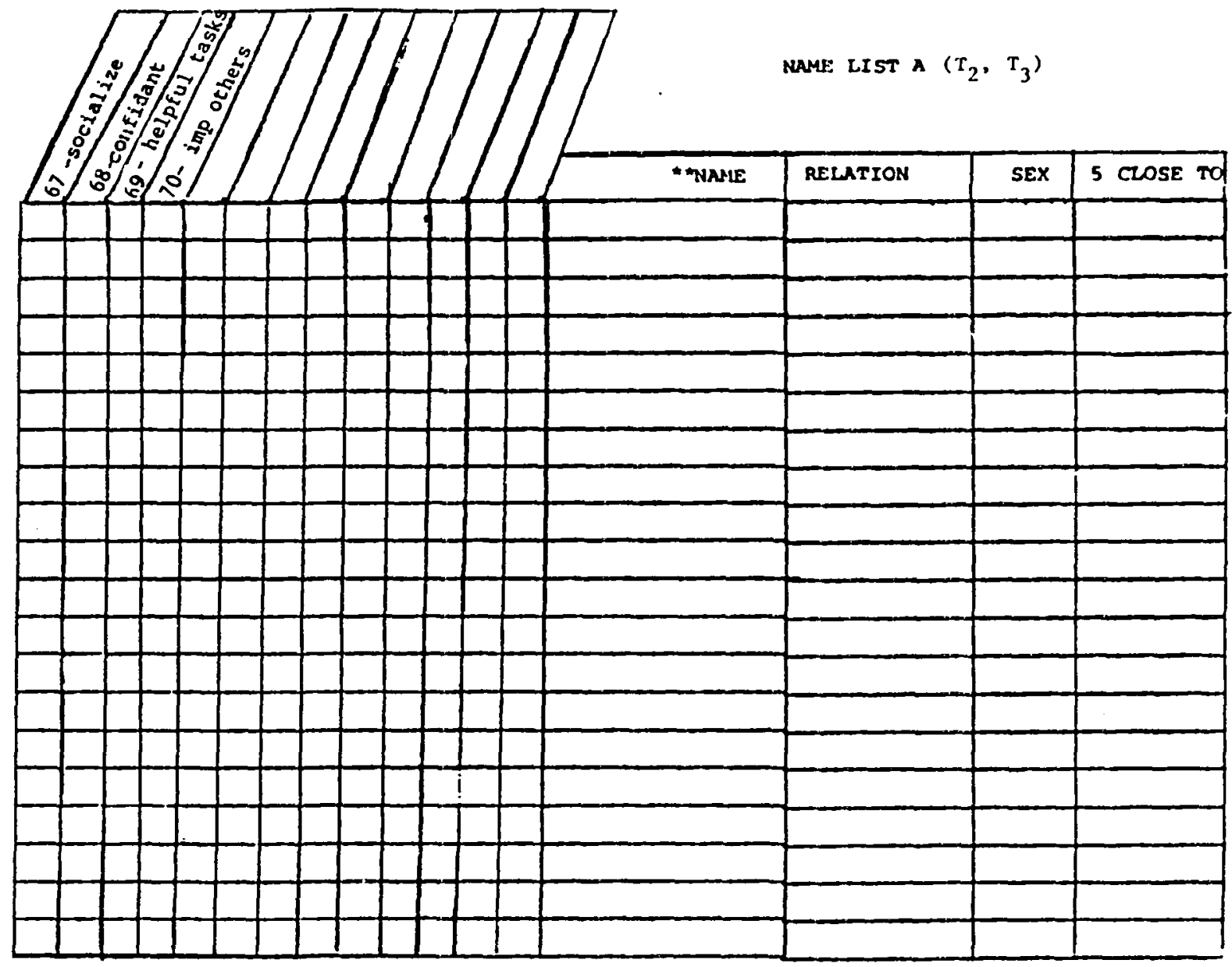

Relationship Code

0 -other

1-apouse

2 -child

3-sibling

4-parent

5-other relative

6-neightor

7-work assocrate

8-friend

Sex code

1 -female

2 male

* genefate a maximum of 20 nahes wit! relation nin sex

INFORMATION ON EACII (FIRST NAIE AND LAST INITIAL) 
(Circle all names that appear on both

\begin{tabular}{|c|c|c|c|c|c|c|}
\hline SV and PI coding sheets & j) & Name & Name & Name & Name & Name \\
\hline & & Age: & Age: & Age: & Age: & Age: \\
\hline $\begin{array}{l}\text { How long have } \\
\text { you known this } \\
\text { person? }\end{array}$ & 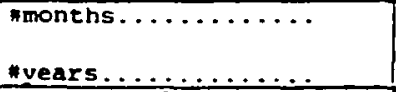 & & & & & \\
\hline $\begin{array}{l}\text { How of ten do you } \\
\text { see this person? }\end{array}$ & 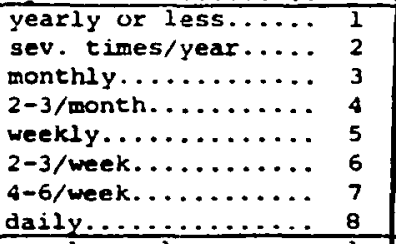 & & & & & \\
\hline $\begin{array}{l}\text { How often do you } \\
\text { talk on the phone } \\
\text { or exchange } \\
\text { letters? }\end{array}$ & 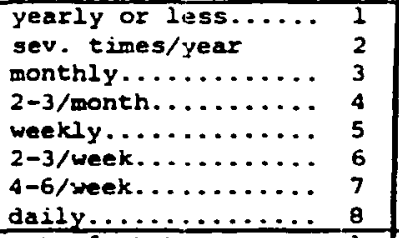 & & & & & \\
\hline $\begin{array}{l}\text { Where does } \\
\text { Iive? }\end{array}$ & 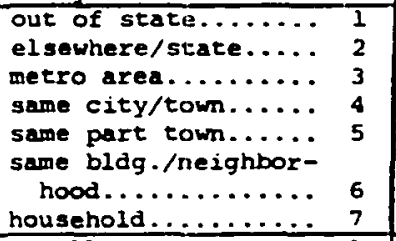 & & & & & \\
\hline $\begin{array}{l}\text { When you get in } \\
\text { touch with } \\
\text { who initiates } \\
\text { contact usually? }\end{array}$ & 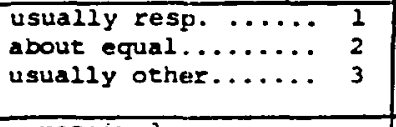 & & & & & \\
\hline $\begin{array}{l}\text { Has } \\
\text { help of any kind from you } \\
\text { month? }\end{array}$ & $\begin{array}{l}\text { of received } \\
\text { ou in the last } \\
\text { No } \ldots \ldots, \ldots, \ldots, \ldots, 0 \\
\text { Yes..., o } \\
\text { Frequency } \\
\text { Specify Type }\end{array}$ & $\begin{array}{l}\text { Freq } \\
\text { Type }\end{array}$ & $\begin{array}{l}\text { Freq } \\
\text { Type }\end{array}$ & $\begin{array}{l}\text { Freq } \\
\text { Type }\end{array}$ & $\begin{array}{l}\text { Freq } \\
\text { Type }\end{array}$ & $\begin{array}{l}\text { Ereg. } \\
\text { Type }\end{array}$ \\
\hline $\begin{array}{l}\text { - If spouse or household } \\
\text { member, delete column } \\
\text { except for age and how } \\
\text { long known. }\end{array}$ & $\begin{array}{l}\text { Support code: } \\
1=\text { affective/emotional } \\
2=1 \text { instrumental } \\
3=1 \text { formation } \\
4=\text { combination }\end{array}$ & & & & & \\
\hline
\end{tabular}


Stroke-Pelated Data $\left(T_{2}, T_{3}\right)$

1. Ongoing Therapies SV Still Receiving:

PT

OT

ST

Other (specify)

Other (spectfy)

Provider(s) of Tx

$0=$ no

1 = yes

2. Has SV been hospitalized for purposes of rehab., or have services related to the stroke been sought on an outpatient basis since last interview?*

$0=$ no

1 × yes

When? (inclusive $* \star$ Service Referred or Inpatient dates) or Tx Factlity/Provider self-sought or outpatient?

*Include evaluations for more rehab. as well.

${ }^{\star \star}$ Examples: day care, driver retraining, recreational $t x$, home health services.

3. Date $d / c$ from hospital or rehab. facility, if not obtained at $T_{1}$

4. Approximate total amount of Rehab. Therapies:

$\overline{\mathrm{PT}}-\frac{\mathrm{OT}}{\mathrm{ST}}-\frac{\text { Other }}{\text { Other }}$


APPENDIX C

OTHER MEASURES 
CES-D1

Here is a list of items of ways you might have felt or behaved. please indicate how often you felt this way during the past week by circling the appropriate response next to each item.

IN THE PAST WEEK, HOW MANY DAYS DID TIIIS HAPPEN TO YOU?

1. I was bothered by things that usually don't bother me.

2. I did not feel like eating, my appetite was poor.

3. I felt that I could not shake of the blues even with help from my friends and family.

4. I felt that I was just as good as other people.

5. I had trouble keeping my mind on what $I$ was doing.

6. I felt depressed.

7. I fe]t that everything I did was an effort.

8. I felt hopeful about the future.

9. I thought my life had been a failure.

10. I felt fearful.

\begin{tabular}{|c|c|c|c|}
\hline $\begin{array}{l}\text { Rarely } \\
\text { or none } \\
\text { of the }\end{array}$ & $\begin{array}{l}\text { Some or } \\
\text { a little }\end{array}$ & $\begin{array}{l}\text { Occasion- } \\
\text { ally or a } \\
\text { moderate }\end{array}$ & Most or \\
\hline $\begin{array}{l}\text { (Less } \\
\text { than } 1 \\
\text { day) }\end{array}$ & $\begin{array}{l}\text { t lme } \\
(1-2 \\
\text { days })\end{array}$ & $\begin{array}{l}\text { of t ime } \\
(3-4 \\
\text { days })\end{array}$ & $\begin{array}{l}\text { the time } \\
(5-7 \\
\text { days })\end{array}$ \\
\hline
\end{tabular}

0

1

2

3

0

1

2

3

0

1

2

3

3

2

1

0

0

1

2

3

0

1

2

0

1

2

3

3

3

2

1

0

0

1

2

3

0

1
3 
CES-D2

\begin{tabular}{lccc} 
Raraly & \multicolumn{3}{c}{ Occasion- } \\
or none & Some or & ally or a \\
of the & a little moderate & Most or \\
time & of the & amount & all of \\
(Less & time & of time & the $t$ ime \\
than 1 & $(1-2$ & $(3-4$ & $(5-7$ \\
day & days $)$ & days $)$ & days $)$ \\
\hline
\end{tabular}

11. My sleep was restless.

0

1

2

3

12. I was happy.

3

2

1

0

13. I talked less than

0

1

2 usual.

14. I felt lonely.

0

15. People were unf rlendly.

0

16. I enjoyed life.

3

17. I had crying spells.

0

18. I felt sad.

19. I felt that people disliked me.

20. I could not get "going."

21. I had trouble falling asleep.

0

0

1

1

2

2

1

2

1

1

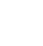

2

3

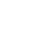

\section{3}

3

3

0

3

3

1

2

3

$\begin{array}{lllll}0 & 1 & 2 & 3 & - \\ 0 & 1 & 2 & 3 & -\end{array}$

22. I felt irritable.

23. I have been worrying a lot. 
CES-D3

24. I wake up in the middle of the night (not to go to the bathroom).

25. I was interested in my usual activities.

26. I slept nuch more than usual.

27. I felt guilty.

28. Did you blame yourself for anything you have done or not done?

\begin{tabular}{|c|c|c|c|}
\hline $\begin{array}{l}\text { Rarely } \\
\text { or none } \\
\text { of the } \\
\text { t lme } \\
\text { (Less } \\
\text { than } 1 \\
\text { day) } \\
\end{array}$ & $\begin{array}{l}\text { Some or } \\
\text { a } 1 \text { ittle } \\
\text { of the } \\
\text { t Ime } \\
(1-2 \\
\text { days }) \\
\end{array}$ & $\begin{array}{l}\text { Occaston- } \\
\text { ally or a } \\
\text { moderate } \\
\text { amouni } \\
\text { of t ime } \\
(3-4 \\
\text { days }) \\
\end{array}$ & $\begin{array}{l}\text { Most or } \\
\text { all of } \\
\text { the time } \\
(5-7 \\
\text { days) } \\
\end{array}$ \\
\hline 0 & 1 & 2 & 3 \\
\hline 3 & 2 & 1 & 0 \\
\hline 0 & 1 & 2 & 3 \\
\hline 0 & 1 & 2 & 3 \\
\hline 0 & 1 & 2 & 3 \\
\hline
\end{tabular}

CES-D Total Score: 
IPWB

Instructions: Here is a list that describes some of the ways people feel at different times. How of ten do jou isei each of these ways?

\begin{tabular}{|c|c|c|c|}
\hline & 0 & 1 & 3 \\
\hline & Never & Sometimes & often \\
\hline $\begin{array}{l}\text { 1. Very lonely or remote from other } \\
\text { people }\end{array}$ & & & \\
\hline 2. Bored & & & \\
\hline 3. On top of the world & & & \\
\hline $\begin{array}{l}\text { 4. Vaguely uneasy about something } \\
\text { without knowing why }\end{array}$ & & & \\
\hline 5. Depressed or very unhappy & & & \\
\hline $\begin{array}{l}\text { 6. Particularly excited or interested } \\
\text { in something }\end{array}$ & & & \\
\hline 7. So restless you had to move about & & & \\
\hline $\begin{array}{l}\text { B. Pleased about having accomplished } \\
\text { something }\end{array}$ & & & \\
\hline
\end{tabular}

N Score

P Score

Bal. Score 
INSTRUCTIONS: Please indicate the level of activity of the stroke patient in your family by circling the appropriate number next to each activity.

Barthel Index l (Maryland Disability Index)

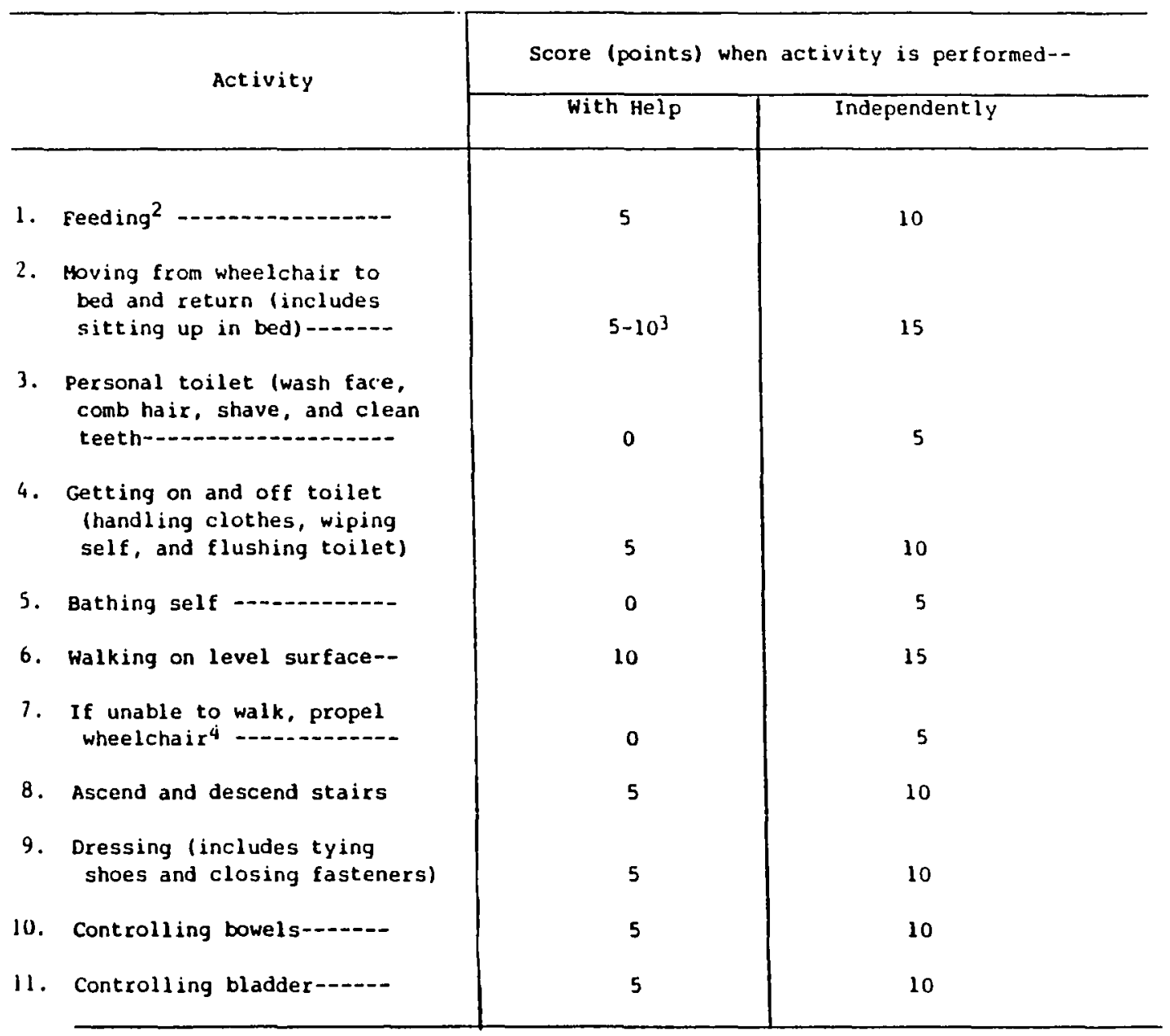

1

Mahoney, F. I., and D. W. Barthel, Functional evaluation: The Barthel Index.

Md. State Medical Journal, 14, 61-65 (1965).

2

If food must be cut up, the pratient is scored as needing help.

3

Score 5 if tranisfors with a great deal of help; score 10 if minimal help is needed.

4 Score only if unable to walk.

Total Barthel Score 
ASFD

PLEASE CHECK THE APPROPRIATE LEVEL OF FUNCTIONING FOR THE ST KOKE PATIENT IN YOUR FAMILY

1. Able to follow directions at the level of a one-step command, e.g., put your hand on your head.

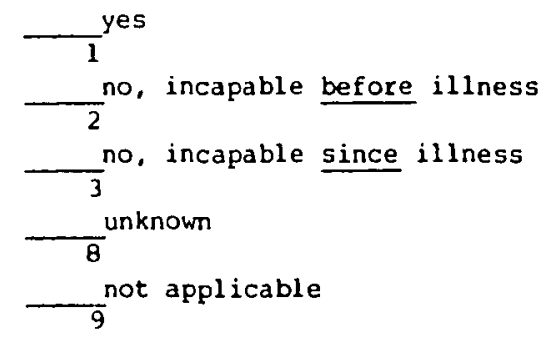

2. Expressive communication: Usual method used to convey information, ideas, and needs $\tau_{1}$ speaks and is usually understood

speaks but is understood only with difficulty

2

uses only structured sign language, or symbol board or writes to communicate 3

no communication

4

unknown

$\frac{9}{9}^{\text {not }}$ applicable

3. Capable of making change for money transactions without assistance from others

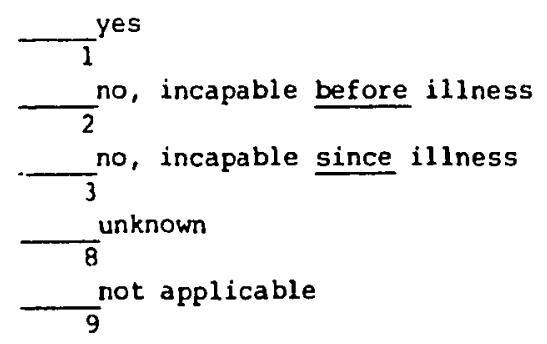

4. Capable of complex arithmetic, like checkbook balancing or taxes, without assistance from others

$T^{\text {yes }}$

no, incapable before illness

2 
4. Continued

$\frac{3}{3}^{\text {no, incapable since illness }}$
$\frac{8}{\text { unknown }}$ not applicable

5. Capable of housework, including simple meal preparation without assistance from others

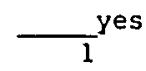

$-_{2}$ no, incapable before illness

]$_{3}^{\text {no, incapable since illness }}$

$\overline{8}^{\text {unknown }}$

$\frac{1}{9}^{\text {not }}$ applicable

6. Manifulates telephone, both dialing and talking

$\mathrm{L}_{1}$ yes, independently

$-\frac{y}{2}$ yes, with assistance or supervision from others

no, incapable before illness

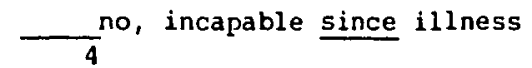

$\tau_{8}^{\text {unknown }}$

]$^{\text {not }}$ applicable

7. Capable of using public or private transportation

]$_{1}$ yes, independently

yes, with assistance or supervision from others

no, incapable before illness

3

no, incapable since illness

4

]$_{8}$ unknown

$\frac{9}{9}^{\text {not applicable }}$ 
Cont inued

8. Capable of performing general outdoor activities which are active (e.g., gardening) and/or passive (e.g., sitting outside)

yes, independently

yes, with assistance or supervision from others

$-_{3}$ no, incapable before illness

$\frac{1}{4}^{\text {no, incapable since illness }}$

unknown

8

$-\frac{-1}{9}^{\text {not }}$ applicable

9. Capable of engaging in formal/informal social activities inside/outside the home

$L_{1}^{y e s,}$ independently

yes, with assistance or supervision from others

2

no, incapable before illness

3

no, incapable since illness

4

unknown

not applicable

9

Total ASFD Score 
Respondent Code

LOT

Please answer the following questions about yourself by circling tae appropriate number after each statement. For each of the statements, inalcate the extent of your agreedent by using the following scale:

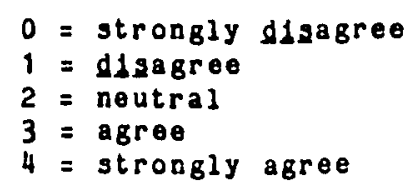

Please be as bonest as you can throughout, and try not to let your responses to one question incluence your response to otber questions. There are no right or wrong answers.

1. In uncertain times, I usually expect tho best. $0 \quad 1 \quad 2 \quad 34$

2. If something oan go wrong for me, it will. $0 \quad 12234$

3. I always look on the bright side of things. $0 \quad 1 \quad 2 \quad 34$

4. I'm always optimistic about my future. 0112334

5. I bardly over expect things to go my way. $0 \quad 1 \quad 2 \quad 34$

6. Things nover work out the way I want them to. $0 \quad 1 \quad 2 \quad 34$

7. I'm a beilever in tho 1dea that "arery eloud has a g1lyer lining." $\begin{array}{lllll}0 & 1 & 2 & 3 & 4\end{array}$

8. I rarely count on good things happening to me. $0 \quad 1 \quad 2 \quad 34$ 
C.BS

Here 1 s a list of stateaents that describe bow people sometimes feel when caring for soveone who bas bad a stroke. After reading each statement, choose the cost appropriate number/word that describes your feelings, from "never" to "almost always."

1. I feel that I don't do as wuch for the stroke patient as I could or should.

_-_Hever _-_Rarely _._Sometimes _-_orten _-_Almost Always

2. I roel the stroke petiont is dependent.

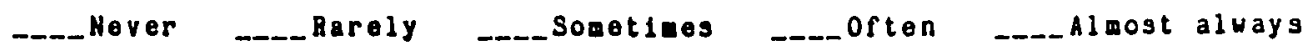

3. I feel that I as contributing to the well-being of the stroke patient. ___ Hever ____Rarely ___ Sometiges ___often ___Almost always

4. I reol that the stroke patient akes requests of me that are over and above what helshe needs.

_-_Never ___Rarely ___Sometimes ___often ___ Almost always

5. Because or ay Involvesent witb the stroke patient, I don't bave enough t1me to aysele.

__- Never __-_Rarely _._. Sometimes _-_often ___Almost always

6. I foel resentful of other relatives who could but who do not do tnings with or for the stroke patient.

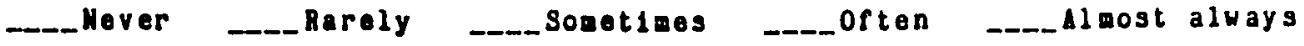

7. I reel stressed botween trying to givo to the stroke patient as well as to other ramily responsibilities, job, eto.

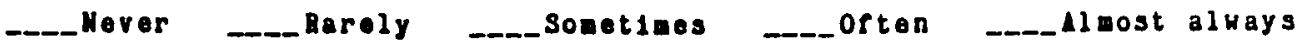


8. I soel that the stroke patient currently affects ay relationships with other fanily mezbers and frionds in a negative way.

_-_Hover __-_Barely ___Soetimes ___often _-_Almost always

9. I reel userul in ay interactions witb the stroke patient.

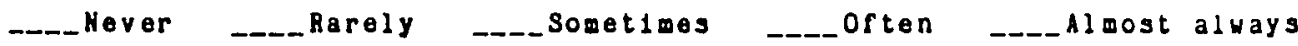

10. I foel that ay boalth has sufforod becauso of my involvement with the stroke patient.

_-_Hever ___Rarely ___Sometimes ___often ___Almost always

11. I feol that my social life bas suffered because of my involvement with the stroke patient.

_-_Hever __-_Rarely _._Sometimes ___often _-_Almost always

12. I reel that the stroke patient doesn't appreciate what I do for him/her as such as I would $11 \mathrm{ko}$.

___ Hover ___ Rarely ___Sometimes ___often ___Almost always

13. I feel that the stroke patient seems to expect me to take care of bialber as if I were the only one helshe could depend on.

_-_Never ___-_Rarely ___Sometimes ___often ____Almogt always

14. I Peel enberrassed over the stroke patient's behavior or condition.

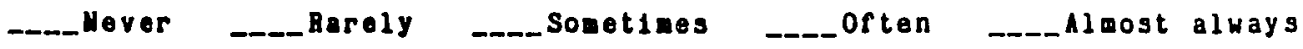


PLEASE CIRCLE THE ANSWER THAT BEST APPLIES TO YOU.

1. I have never intensely disliked anyone.

$$
1=\text { true }
$$

$2=$ false

2. I sometimes feel resentful when I dor.'t get my way.

1 = true

$2=$ false

3. I'm always willing to admit it when I make a mistake.

$1=$ true

2 = false

4. I sometimes try to get even rather than forgive and forget.

$1=$ true

2 = false

5. At times I have really insisted on having things my own way.

1 = true

2 = false

6. I have never been irked when people expressed ideas very different from my own.

1 = true

2 = false

Total SD Score 
APPENDIX D

INFORMED CONSENT 


\author{
STROKE PROJECT \\ PORTLANO STATE UNIVERSITY INSTITUTE ON AGING \\ Informed Consent Form \\ (Primary Informant)
}

I have been dsked to particlpate in the study, "Soclal Network and Hell-Being Changes Caused by a Disabling Stroke", which is being conducted by Richard Schulz, Ph. D., Principal Investigator, and Marle T. Rau, Profect Director, of the Institute on Aging at Portland State University.

The purpose of the study is to learn more about the social support systems of persons who have had a stroke and their families, how the nature and amount of support may change over time, and how these changes in social support are related to the sense of wellbeing experlenced by stroke victims and thelr familles. It is hoped that this research will help us to learn more about the psycho-social aspects of stroke, and what role an Individual's soclal support system plays in recovery from a stroke.

I wlll be asked to respond three times to a questlonnaire about those persons and types of social support which are important in my life. There will also be some questions regarding my current health and life sltuation as I view It. I understand that I will be interviewed in person or by telephone, and that these interviews will take place in my home or in the hospital if inperson. I will be reimbursed for mileage costs which might result from the completion of these interviews. The first interview will take place 3 to slx weeks after my stroke. The second Interview w11l be held 6 months after the first interview, and the third intervlew w11 take place o months after the second interview. Each interview should take about an hour of my $t$ Ime.

I also understand that my who has suffered the stroke may be asked to respond to some of the same, or similar questions. I agree to allow persons conducting this research to obtain certain Information regarding my stroke from hls/her physiclan, theraplsts, and/or frommedical records, wh his/her physiclan's approval. I understand that at all times our right to privacy regarding any of the study information will be strictly safeguarded and protected. Nelther I nor my w 111 be personally Identifled on any of the questionnatre forms, in any of the data analysis, or in any published reports of the study.

I have been assured that participation in this study should involve little risk or discomfort. I may discontinue my participation in the study at any time if I find any part of the procedure to be uncomfortable or objectionable. I understand that elther Dr Schulz or Mrs. Rau (Institute on Aging, phone: 229-3952) w1ll be happy to answer any questions I may have about the project. 


\section{Informed Consent (P. I.)}

I understand that $I$ am free to refuse to particlpate or to withdraw from participation in this study at any time.

I have carefully read or had read to me and understand the foregoing. I hereby voluntarlly consent to my participation $n$ the experimental procedures as described above.

Name of Subject

Signature of Subject Date

Name of WItness

STgnature of Witness Date

Name of Person obtalning Signatures 


\author{
STROK: PROJECT \\ PORTLAND STATE UNIVERSITY INSTITUTE ON AGING \\ Informed Consent Form \\ (Stroke Victim)
}

I have heen asked to partlclpate in the study. "Social Network and well-Being Changes Caused by a Disabling Stroke", which is beling conducted by Rlchard Schulz, Ph. D., Principal Investigator, and Marle T. Rau, Project Director, of the Institute on Aqing at Portland State University.

The purpose of the study is ro learn more about the soclal support systems of persons who have had a stroke and their families, how the nature and amount of support may change over time, and how these changes in sociai support are related to the sense of well-belng experienced by stroke victims and their familles. It is hoped that this research will help us to learn more about the psycho-social aspects of stroke, and about what role an Individual's social support system plays in cecovery from a stroke.

I will be asked to respond two or three times to a questionnaire abnut those persons and types of social support which are imfortant in my life. There will also be some questions regarding my current health and 1 ife situation as I view it. I understand that I will be interviewed in person or by telephone, and that these interviews if in-person will take place in my home or in the hospltal. I wlll be relmbursed for mileage costs which might result from the completion of these interviews. The first interview wlll take place 3 to 6 meeks after the stroke. The second interview wlll be held 6 months after the first interview, and the third interview will take place 6 months after the second interview. Each interview should take about an hour of my $t$ ime.

I also understand that an important other person in my life (spouse, chlld, close friend or other relative) will be asked to answer some of the same, or $51 \mathrm{mll}$ ar questions.

I aqree to allow persons conducting this research to obtain certain information regarding my stroke from my phystcian, theraplsts, and/or medical records, with my physician's approval. I understand that at all times my right to privacy regarding any of the study Information will be strictly safeguarded and protected. I will not be personally identified on any of the questionnalre forms. In any of the data analysis, or in any published reports of the study.

I have been assured that partlcipation in this study should involve little risk or discomfort. I may discontinue my participation in the study at any time If I find any part of the procedure to be uncomfortable or objectionable. I understand that 


\section{Informed Consent (S. V.)}

elther Dr. Schulz (phone 229-3952) or Mrs. Rau (phone

229-3952) will be happy to answer any questions I may have about the project.

I understand that I am free to refuse to partlcipate or to withdray from participation in this study at any time without effect on my relationship with or treatment

I have carefully read or had read to me and understand the foregoing. I hereby voluntarlly ronsent to my particifation in the experimental procedures as descrlbed above.

Name of Subject

STgnature of Subject Date

Name of WIfness

SIgnature of WItness Date

Name of Legal Cuardian of Suhject (if applicable)

SIgnature of Guardian Date

Name of Person obtaining Signatures 